\title{
Review on the Third International Neuroinflammation Congress and Student Festival of Neuroscience in Mashhad University of Medical Sciences
}

\author{
Sayed Mostafa Modarres Mousavi ${ }^{1}$, Sajad Sahab Negah², Ali Gorji ${ }^{1,3^{*}}$ \\ ${ }^{1}$ Shefa Neuroscience Research Center, Khatam Alanbia Hospital, Tehran, Iran \\ ${ }^{2}$ Department of Neuroscience, Mashhad University of Medical Sciences, Mashhad, Iran
}

${ }^{3}$ Epilepsy Research Center, Department of Neurology and Neurosurgery, Westfälische Wilhelms-Universität Münster, Münster, Germany

\section{A BSTRACT}

Introduction: Neuroinflammation congress was the third in a series of annual events aimed to facilitate the investigative and analytical discussions on a range of neuroinflammatory diseases. The neuroinflammation congress focused on various neuroinflammatory disorders, including multiple sclerosis, brain tumors, epilepsy, and neurodegenerative diseases. The conference was held in June 11-13, 2019 and organized by Mashhad University of Medical Sciences and Munster University, which aimed to shed light on the causes of neuroinflammatory diseases and uncover new treatment pathways. Conclusion: Through a comprehensive scientific program with a broad basic and clinical aspects, we discussed the basic aspects of neuroinflammation and neurodegeneration up to the state-of-the-art treatments. In this congress, 334 scientific topics were presented and discussed.

Key words:

1. Nervous System

Diseases

2. Inflammation

3. Congress

4. Iran

5. Germany

*Corresponding Author: Ali Gorji

E-mail: gorjial@uni-muenster.de 


\title{
مرورى بر سومين همايش بين المللى التهاب سيستم عصبى و سومين فستيوال دانشجويى علوم اعصاب
}

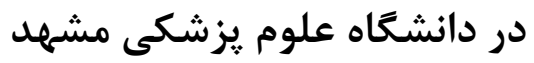

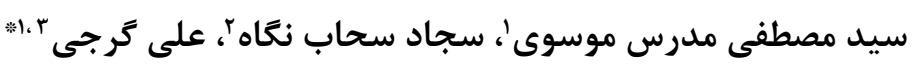 \\ 'مركز تحقيقات علوم اعصاب شفا، بيمارستان خاتمالانبيا، تهران، ايران

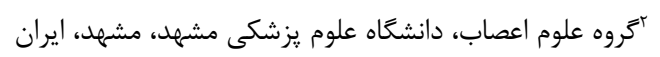

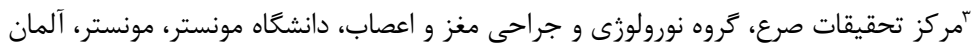

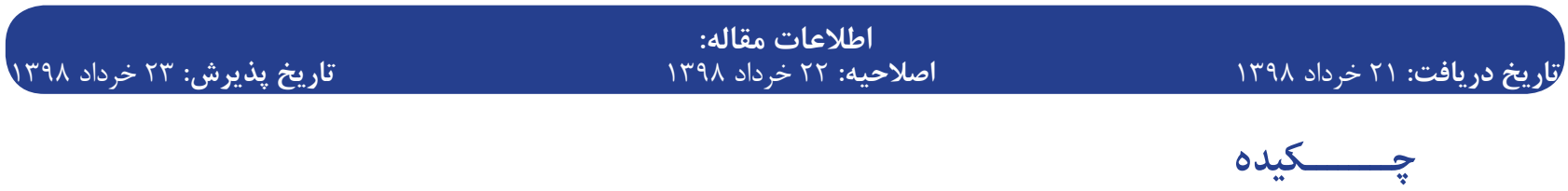

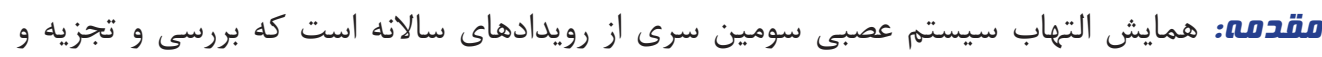

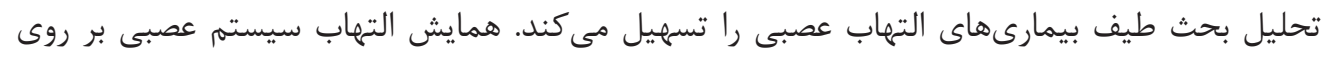

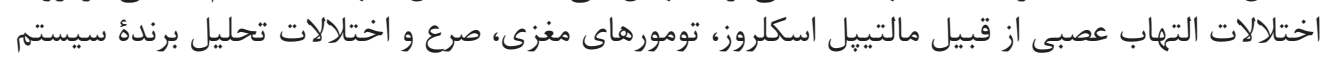

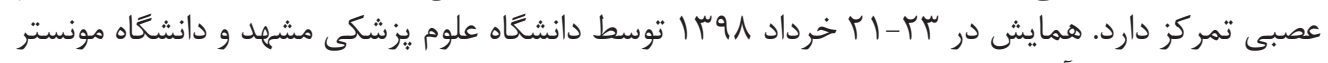

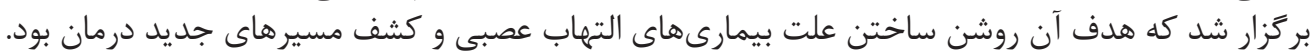

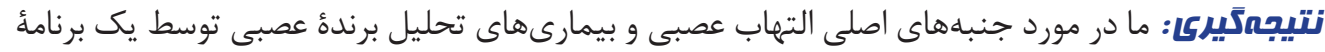

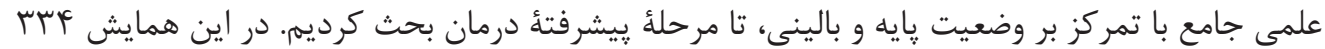

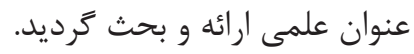




$$
\text { نتيجهَ }
$$

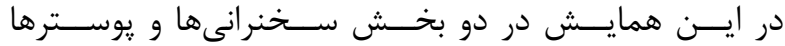

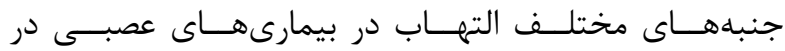

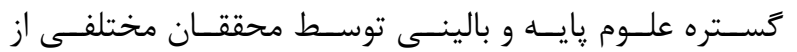

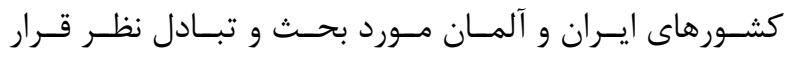

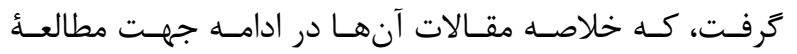

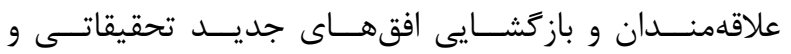

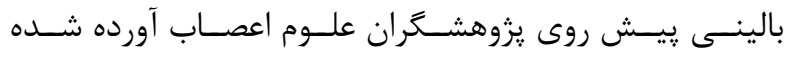

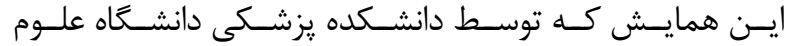

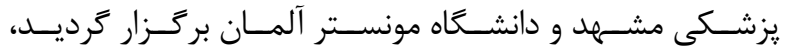

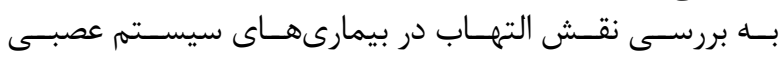

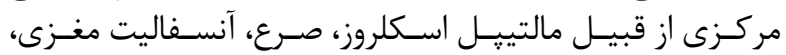

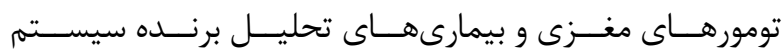

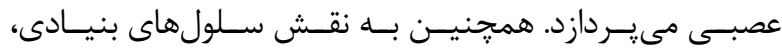

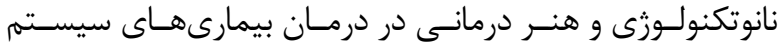

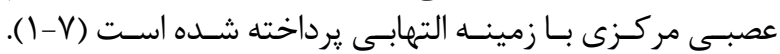

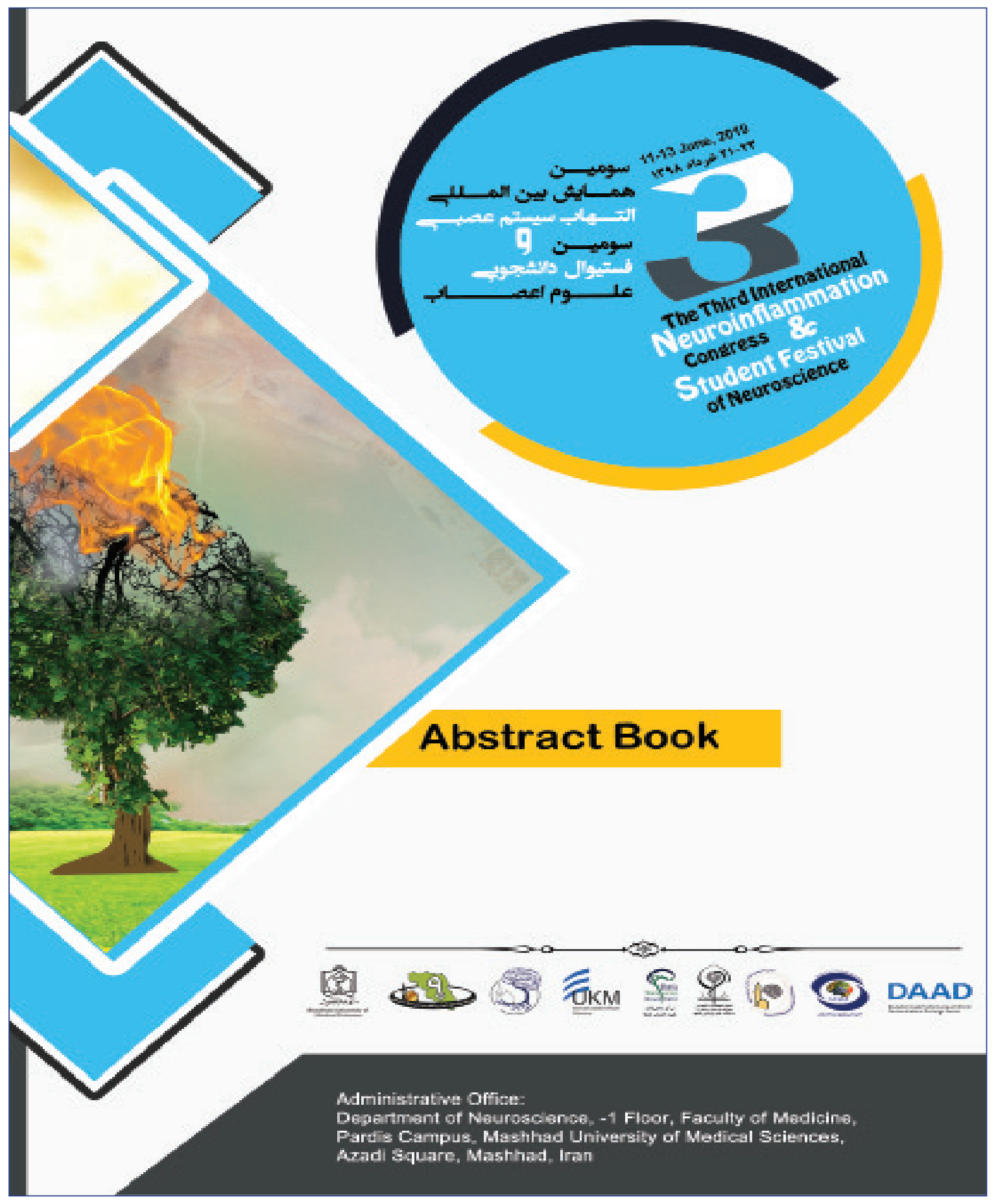




\title{
The Neuroscience Journal of Shefaye Rhatam
}

\section{Abstract Book of The $3^{\text {rd }}$ International Neuroinflammation Congress \& The $3^{\text {rd }}$ Student Festival of Neuroscience}

\author{
11-13 June, 2019, Mashhad, Iran.
}

\section{Oral Presentations}

\section{1}

Evaluation of Effects on Improvement of Muscle Strength, Prevention of Oligodendrocytes and Myelin Damage in Brain, Animal Model of MS Disease

\section{Alireza Lotfi ${ }^{1}$, Nazem Ghasemi ${ }^{2}$, Zahra Abroodi ${ }^{2}$}

Department of Anatomical Sciences, Faculty of Medicine, Isfahan University of Medical Sciences, Isfahan, Iran

The Neuroscience Journal of Shefaye Khatam, 2019; 7(3): O1

multiple sclerosis (MS) is a disorder with autoimmune pathophysiology which is accompanied by neurological disability. Astaxanthin (AST) can be effective in prevention of this abnormal conditions because of its ability to cross the blood brain barrier and the other reasons are antioxidant and nerve protection effect. The purpose of this study is to evaluate AST effect on MS prevention by appraising the muscle strength, prevention of oligodendrocyte cells and myelin degradation in Rat brain. 40 wistar rats were divided into four experimental groups: control, cuprizone, sham (cuprizone + DMSO) and astaxanthin. AST group received cuprizone $(0.6 \%)$ and AST $(3 \mathrm{mg} / \mathrm{Kg})$ coincidently for four weeks by gavaging. During the study we evaluate muscle strength by using a behavioral basket test, the percentage of cell expressing A2B5 and MOG markers by immunohistochemistry technique. Myelin density was evaluated by luxal fast blue staining. We use RT PCR in order to measuring MBP, MOG and PDGFr gene expression. Using Image J and SPSS softwares, the results were analyzed by one-way ANOVA test. our data in immunochemistry and other evaluation techniques showed that the percentage of mature oligodendrocytes and oligodendrocyte progenitor cells in the AST group were remarkable higher than those in the sham and cuprizone group. In addition, myelin density and muscle strength in AST group were higher than the those cuprizone and sham group. $(\mathrm{p} \leq 0.05)$. this study confirms the positive effects of AST and natural compounds containing AST in the prevention of oligodendrocytes and myelin ddestruction in the people susceptible to MS.
02

Cytotoxic T-Cells Cause Hippocampal Sclerosis in an Animal Model of Autoimmune Limbic Encephalitis

Marco Gallus ${ }^{1 *}$ Andre Dik ${ }^{1}$, Veno Narayanan ${ }^{1}$, Gordon Hicking ${ }^{1}$, Tobias Ruck1, ManuelaCerina ${ }^{1}$, Lydia Wachsmuth', Cornelius Faber ${ }^{2}$, Sven G Meuth ${ }^{1}$, Nico Melzer ${ }^{1}$

${ }^{1}$ Institute of Translational Neurology, Department of Neurology, University of Münster, Münster, Germany

${ }^{2}$ Department of Radiology, University of Münster, Münster, Germany

The Neuroscience Journal of Shefaye Khatam, 2019; 7(3): O2

Autoimmune limbic encephalitis (ALE) describes group of autoimmune inflammatory syndromes, often associated with cancer and characterized by neurocognitive impairment, psychiatric symptoms like depression and mesial temporal lobe epilepsy. Magnetic resonance imaging (MRI) initially shows an edema with swelling and T2-signal increase of the amygdala and hippocampus which subsequently may turn into hippocampal sclerosis with atrophy and persisting T2signal increase. In some patients autoantibodies against intracellular neural antigens can be detected in serum and cerebrospinal fluid. In these cases, cytotoxic $\mathrm{T}$ cells (CTL) are thought to cause lysis of neurons which liberate intracellular antigens that trigger a $\mathrm{B}$ cell response with formation of autoantibodies. To better understand the pathophysiological mechanisms, the goal of this study was to develop a CTL-mediated animal model of ALE. To that aim, we took advantage of the Combined Adjuvant for Synergistic Activation of Cellular immunity (CASAC) to induce a CTL-mediated answer against ovalbumin expressing hippocampal CA1 neurons, created via stereotaxic injection of an adenovirus vector encoding for ovalbumin or a control protein under the control of a synapsin promoter. Immunohistochemical staining showed infiltrations of CTLs in the hippocampus leading to astrocytosis, microglia activation and neuronal death. 
This corresponds to an antigen-dependent hippocampal atrophy as demonstrated by MRI and memory disturbance as revealed by the Novel Object Recognition Test in mice. Taken together, we demonstrate that CTL can cause hippocampal sclerosis in an animal model of ALE.

\section{O3}

\section{Art and Epilepsy}

\section{Shaghayegh Gorji}

Epilepsy Research Center, Munster University, Munster, Germany

The Neuroscience Journal of Shefaye Khatam, 2019; 7(3): O3

Epilepsy is one of the most common neurological disorders, affecting approximately 50 million people worldwide. Epilepsy is associated with misunderstanding and social stigma among different nations. Art improves understanding of epilepsy not only in public, but also among physicians, students, and scientists of what it means to have seizures and helps to alleviate stigma. Art may also help to improve student's observational skills and bedside manners and inspire clinicians and researchers to further investigations the basic mechanisms and novel therapeutic approaches.

\section{4}

Anti-Inflammatory Effects of Ursin Administration in Chemical Kindling Model of Epilepsy

\section{Seyed Raheleh Ahmadian', Mahdi Pouramir ${ }^{2}$, Maryam Ghasemi ${ }^{3}$, Farzin Sadeghi ${ }^{3}$}

${ }^{1}$ Student Research Committee, Babol University of Medical Sciences, Babol, Iran

${ }^{2}$ Cellular and Molecular Biology Research Institute, Faculty of Medicine, Babol University of Medical Sciences, Babol, Iran ${ }^{3}$ Cellular and Molecular Biology Research Center, Health Research Center, Babol University of Medical Sciences, Babol, Iran

The Neuroscience Journal of Shefaye Khatam, 2019; 7(3): O4

Epilepsy is one of the most common chronic neurological disorders, which provoke progressive neuronal degeneration and memory impairment. In recent years, application of herbal compounds with anti-inflammatory properties, such as ursin has been introduced as useful agent in reducing of the epilepsy symptoms. In this study, the effect of ursin application on spatial memory improvement, neuronal density and astrocyte activation were evaluated in pentylenetetrazol (PTZ)-induced kindling model. Male NMRI mice have received the daily injection of ursin at dose of 25 and $50 \mathrm{mg} / \mathrm{kg}$. All interventions were injected intraperitoneally (i.p.), 10 days before PTZ administration and the injections were continued until $1 \mathrm{~h}$ before each PTZ injection.
Spatial learning and memory was evaluated using Morris water maze test after the 11th PTZ injection. Animals have received 13 injections of PTZ and then, brain tissues were removed. Immunostaining against NeuN and GFAP respectively as mature neuronal and astrocyte markers were performed on brain sections. High performance liquid chromatography (HPLC) was performed to measure the ursin content in hippocampus. Our results showed that ursin administration reduced the seizures behavior and prevented cognitive impairment in fully kindled animals. The results of HPLC analysis showed that high amount of ursin can be detected in hippocampus of ursin+PTZ receiving animals. Immunostaining data demonstrated that the level of neuronal loss and astrocyte activation were reduced in animals under treatment of ursin. Overall, the results of this study suggest that ursin administration effectively ameliorates memory impairment and alleviates the level of neuronal death and astrocyte activation in PTZinduced kindling model.

\section{O5}

A Polymeric Nanoparticle Formulation of BMIInhibitor Decreased Growth and Migration of Glioblastoma Stem-Like Cells

\section{Sajad Sahab-Negah, Elham Poonaki, Fatemeh Ariakia, Mohammad Jalili-Nik, Farzane Abbasi Nezhad}

Neuroscience Research Centre, Mashhad University of Medical Sciences, Mashhad, Iran

The Neuroscience Journal of Shefaye Khatam, 2019; 7(3): O5

Glioblastoma multiform (GBM) is the most malignant form of brain tumors in adults. Recent achievements have shown that cancer therapy failure, GBM recurrence and short life survival in these patients are associated with existence of self-renewing CD133 positive cancer stem cells (CSCs) in GBM. In this study, PTC-209 (BMI-1 inhibitor) utilized to load into PLGA-PEG nanoparticle conjugated with $\mathrm{CD} 133$ as a targeted delivery to suppress growth of CSCs in the GBM. Primary GBM cells derived from two patients who suffered from GBM and U87 as a GBM cell line have been cultured in this study. Cells were treated based on IC50 treated by polymeric nanoparticle of BMI-inhibitor in different doses. Proliferation, viability, and migration were assessed by MTT, live/dead, and wound healing assay, respectively. our results showed that polymeric nanoparticle formulation of BMI-inhibitor significantly decreased proliferation, viability, and migration of CSCs. BMI-1 inhibitor delivered by PLGA may have great potential for eradicating CSCs in the future if further experiments could confirm these achievements. 
06

The Cannabinoid Receptors Agonist, $\beta$-Amyrin, Modulates LPS/IFN-y Induced M1/M2 Microglia Imbalance

\section{Vahid Reza Askari', 2, Vafa Baradaran Rahimi ${ }^{3}$}

${ }^{1}$ Pharmacological Research Center of Medicinal Plants, Mashhad University of Medical Sciences, Mashhad, Iran

${ }^{2}$ Neurogenic Inflammation Research Center, Mashhad University of Medical Sciences, Mashhad, Iran

${ }^{3}$ Student Research Committee, Department of Pharmacology, Faculty of Medicine, Mashhad University of Medical Sciences, Mashhad, Iran

The Neuroscience Journal of Shefaye Khatam, 2019; 7(3): O6

Inflammation is a primary response to infection that can pathologically lead to various diseases including neurodegenerative diseases. The purpose of this study was to evaluate the effect of $\beta$-Amyrin, a naturally occurring pentacyclic triterpenoid compound, on inflammation induced by lipopolysaccharide (LPS) and interferone- $\gamma$ (IFN- $\gamma)$ in rat microglial cells. Cytotoxicity of $\beta$-Amyrin (3-100) $\mu \mathrm{M}$ on microglial cells was evaluated using the MTT assay. Also, the protective effect of various $\beta$-Amyrin $(2-16 \mu \mathrm{M})$ concentrations with LPS/IFN- $\gamma$-induced mice microglial cells was studied. The concentrations of TNF- $\alpha$ (Tumor Necrosis Factor- $\alpha$ ), IL-1 $\beta$ (Interleukin$1 \beta)$, IL-6 (Interleukin-6) and PGE-2 (Prostaglandin E2) were evaluated using ELISA. Gene expressions of TNF- $\alpha$, IL-1 $\beta$, IL-6, COX-2 (Cyclooxygenase-2), iNOS and arginase- 1 were also evaluated using the Real-Time PCR method. Nitrite oxide and urea were measured using biochemical methods. The studied concentrations of $\beta$-Amyrin had no significant effects on the viability of microglial cells. Interestingly, $\beta$-Amyrin concentration dependently and significantly increased the reduced cell proliferation concerning to LPS/IFN- $\gamma$ exposure $(p<0.001)$. The concentrations and expression $1 \mathrm{e}$ vels of pro-inflammatory factors including TNF- $\alpha$, IL-1 $\beta$, IL-6, PGE-2, COX-2 were significantly re $\mathrm{d}$ uced after $\beta$-Amyrin treatment in LPS/IFN- $\gamma$-induced microglial cells $(\mathrm{p}<0.05-0.001)$. $\beta$-Amyrin also $d$ e creased the levels of nitric oxide, increased urea and down regulated the expression of nitric oxide synthesis while arginase-1 expression was enhanced $(p<0.001)$. The ratio of NO/urea and iNOS/ $\operatorname{Arg} 1$ were also markedly increased in comparison to the LPS/IFN-g group $(p<0.001)$. $\beta$-Amyrin reduces inflammation in microglial cells and can be used as a potential anti-inflammatory agent in central nervous system neurodegenerative diseases such as Alzheimer and multiple sclerosis, by affecting the inflammatory cytokine and differentiation of microglia as resident CNS macrophages.
O7

New Pathophysiological Principles in Autoimmune Neurodegeneration

\section{Sven Meuth}

Department of Neurology with Institute of Translational Neurology, University Hospital Münster, Münster, Germany

The Neuroscience Journal of Shefaye Khatam, 2019; 7(3): O7

Here I will review our recent findings and papers concerning inflammation and coagulation, ion channel mediated mechanisms of inflammation, neuronal consequences of de- and demyelination as well as neuronal network alterations in animal models of inflammation.

\section{8}

Transplantation of Human Meningioma Stem Cells Loaded on a Self-Assembling Peptide Nanoscaffold Containing IKVAV Improves Traumatic Brain Injury in Rats

Sajad Sahab Negah ${ }^{1,2}$ 2, 3, Pardis Oliazadeh, 2, Ali Jahanbazi Jahan-Abad ${ }^{3}$, Arezou Eshaghabadi ${ }^{3}$, Fariborz Samini ${ }^{4}$, Sepideh Ghasemi ${ }^{3}$,AmirAsghari ${ }^{5}$,Ali Gorji ${ }^{1,2,3,6,7,8}$

${ }^{1}$ Department of Neuroscience, Faculty of Medicine, Mashhad University of Medical Sciences, Mashhad, Iran

${ }^{2}$ Neuroscience Research Center, Mashhad University of Medical Sciences, Mashhad, Iran

${ }^{3}$ Shefa Neuroscience Research Center, Khatam Alanbia Hospital, Tehran, Iran

${ }^{4}$ Department of Neurosurgery, Faculty of Medicine, Mashhad University of Medical Sciences, Mashhad, Iran

${ }^{5}$ Department of Neurocognitive Sciences, Psychiatry and Behavioral Sciences Research Center, Mashhad University of Medical Sciences, Mashhad, Iran

${ }^{6}$ Department of Neurosurgery, Westfälische Wilhelms-Universität Münster, Münster, Germany

${ }^{7}$ Department of Neurology, Westfälische Wilhelms-Universität Münster, Münster, Germany

${ }^{8}$ Epilepsy Research Center, Westfälische Wilhelms-Universität Münster, Münster, Germany

The Neuroscience Journal of Shefaye Khatam, 2019; 7(3): O8

The insufficient regenerative ability of the central nervous system has been found to induce a serious brain functional and structural impairment following traumatic brain injury (TBI). This experiment was aimed to investigate the effect of transplantation of the human meningioma stem-like cells (hMgSCs) seeded in a threedimensional scaffold (RADA4GGSIKVAV; R-GSIK) on the brain functional recovery and neuro-inflammatory responses following TBI in rats. After induction of TBI, the hMgSCs seeded in R-GSIK were transplanted within the injury site and the effects were compared 
to control groups. Co-administration of hMgSCs with R-GSIK led to a remarkable functional recovery after TBI. It was demonstrated that the transplantation of hMgSCs with R-GSIK scaffold considerably enhanced the number of $\mathrm{hMgSC}$ s observed in the brain; while significantly decreased the lesion volume, reactive gliosis, and apoptosis at the injury site in comparison to control groups. Additionally, the expression of Tolllike receptor 4 and its downstream signaling molecules was interestingly inhibited in $\mathrm{hMgSC} / \mathrm{R}-\mathrm{GSIK}$ treated animals. According to the results, it can be inferred that the treatment with hMgSCs seeded in R-GSIK promotes the brain functional recovery after TBI, potentially through the amelioration of inflammatory responses.

\section{O9}

\section{Atypical Features of MS and NMOSD}

\section{Mohammad Ali Nahayati}

Mashhad University of Medical Sciences, Mashhad, Iran

The Neuroscience Journal of Shefaye Khatam, 2019; 7(3): O9

some MS and NMOSD patients with atypical MRI findings have been identified, leading to prolonged delay to diagnosis and high costs to look for alternative diagnoses (i.e. leukodystrophy and small vessel disease). besed on the pervious knowledge if we choose the wrong drug for them their disease may aggrevate. based on review article we established a five groups classification in atypic MS: tumefactive demyelinating lesion (TDL)-onset MS, acute disseminated encephalomyelitis (ADEM)-like MS, cavitary MS and leukodystrophy-like MS. All the patients meeting our radiological criteria for atypical MS were included.and some atypical NMOSD patients. we collect all atypical feature of MS and NMOSD MRI features. Patients in the TDL-onset and ADEMlike groups globally presented an acute onset and a relapsing-remitting evolution. Conversely, patients in the cavitary and leukodystrophy- groups largely evolved with a progressive and severe course. Some NMOSD patient were reported with diffuse pattern. Our goal is to ascertain some atypical MRI feature of demyelinating and astrocytopathic disease to select the best treatment in the early phase of disease. All neurologist involved in demyelinating disorder must be aware of this atypical features.

\section{0}

\section{Key Point in Selecting Treatment in MS}

\section{Sareh Shahmohammadi}

Shahid Beheshti University of Medical Sciences, Tehran, Iran

The Neuroscience Journal of Shefaye Khatam, 2019; 7(3): O10
Multiple sclerosis (MS) is a demyelinating disease of the central nervous system in which inflammation and degeneration simultaneously from the onset of disease, lead to attacks and progression of disability over time. Regarding to the efficacy and safety of MS drugs in various clinical trials, they are classified into the first line (moderate efficacy) and second or even third line (high efficacy) medication. An important subgroup of patients experience severe nature of disease as highly active MS or treatment failure as breakthrough disease and need to treat with high efficacy disease modifying drugs (DMDs) including natalizumab, alemtuzumab, ocrelizuma, fingolimod and cladribine. We reviewed clinical trials and meta-analyses and expert opinions in the literature for high efficacy DMDs. Although, so far there is no definitive algorithm or established consensus for proper patient selection. The lack of head to head clinical trials and different definitions of treatment failure and highly active disease could explain the absence of treatment guideline in these patients. Disease severity, drug efficacy against the safety, patient's preference and adherence, monitoring of adverse events, patient age and comorbidities are important points of treatment decision making.

\section{1}

\section{MS and Other Auto Immune Diseases Coincidence}

\section{Behnaz Sedighi}

Neurology Research Center, Kerman University of Medical Sciences, Kerman, Iran

The Neuroscience Journal of Shefaye Khatam, 2019; 7(3): O11

Comorbid autoimmune diseases in MS patients have been studied extensively with controversial results. Multiple sclerosis (MS) is a complex autoimmune disease arising as a result of complex interactions between genetic and environmental factors. The fact that it shares susceptibility genes with other autoimmune diseases raises the question as to whether MS co-occurs more frequently with these diseases than in the general population. the autoimmune comorbidities evaluated included: alopecia areata, ankylosing spondylitis, autoimmune thyroid disease, bullous pemphigoid, celiac disease, dermatomyositis, idiopathic thrombocytopenic purpura, inflammatory bowel disease, myasthenia gravis, pemphigus vulgaris, pernicious anemia, polymyositis, primary adrenocortical insufficiency, primary biliary cirrhosis, psoriasis, rheumatoid arthritis, Sjogren's syndrome, systemic lupus erythematosus, systemic sclerosis, uveitis, vitiligo, and Wegener's granulomatosis. the most prevalent comorbid autoimmune diseases were psoriasis and thyroid disease. Considering all studies, most autoimmune conditions affected fewer than $5 \%$ of individuals with MS with the possible exceptions of celiac disease, type I diabetes, 
thyroid disease, uveitis and psoriasis for which at least one estimate was over $5 \%$. possible explanations for co-existence of two or even more immune mediated diseases shared susceptibility genes and common causative environmental factors. Although genome screens have failed to identify any common loci between MS and other autoimmune disorders, clustering of nonmajor histocompatibility complex (MHC) susceptibility loci may be another explanation for this phenomenon. Actually regarding nonMHC susceptibility genes a lot of the identified autoimmunity loci seems to be shared among multiple autoimmune diseases.

\section{2}

A Retrospective Comparison of Plasma Exchange Versus Corticosteroids in Acute Relapsing Multiple Sclerosis

Leoni Lammerding ${ }^{1}$, Steffen Pfeuffer ${ }^{l}$, Tobias Ruck', Marcus Brand', Matthias Schilling ${ }^{1}$, Heinz Wiendl $^{1}$, Christoph Kleinschnitz, Sven G. Meuth ${ }^{1}$

${ }^{1}$ Department of Neurology, University of Münster, Münster, Germany

${ }^{2}$ Department of Medical, University of Münster, Münster, Germany

${ }^{3}$ Department of Neurology, University Duisburg-Essen, Essen, Germany

The Neuroscience Journal of Shefaye Khatam, 2019; 7(3): O12

Treatment of acute multiple sclerosis (MS) relapses has remained largely unaltered for decades. Standard treatment for acute relapses is high-dose intravenous (methyl-) prednisolone (IVMPS, 1000mg per day given for 3-5 days). However, approximately one quarter of patients remain with significant disability after one course of IVMPS. For treatment of these patients, apart from extending steroid treatment, apheresis treatments were considered as an alternative in MS relapses. Unfortunately, data on comparison of both regimens are still missing. We set up a retrospective analysis to investigate the efficacy of TPE vs. increased dosages of IVMPS in steroid refractory MS relapses, including patients being treated between January, 2013 and December, 2016. In-depth medical-chart review was performed. Baseline data were compared and treatment outcomes were analyzed using logistic regression models. 193 patients were identified. 99 received a second course of IVMPS as first escalation treatment and 46 were directly subjected to TPE. 53 Patients received TPE after their second course of IVMPS. Baseline characteristics between different treatment groups were evenly balanced. After TPE, a higher proportion of patients experienced marked or full response and these effects were preserved at follow-up. However, those effects vanished, if TPE was performed after two courses of IVMPS and this effect was apparently independent of time to initiation of apheresis treatment. Our findings indicate that early TPE has particular advantages compared to a second course of IVMPS. However, data from prospective, randomized trial in a well-defined cohort are desirable. Classification of Evidence: These findings provide Class IV evidence that for patients, refractory to a first course of IVMPS, TPE has particular advantages compared to a second course of IVMPS.

013

MSCT and HSCT in Multiple Sclerosis

\section{Seyed Masood Nabavi}

Cell Science Research Center, Royan Institute for Stem Cell Biology and Technology, ACECR, Department of Regenerative Biomedicine, Tehran, Iran

The Neuroscience Journal of Shefaye Khatam, 2019; 7(3): O13

The advances in technology of stem cells in the recent decades opened an opportunity window to consider different types of these cells as a kind of therapy for some chronic disabling neurological diseases. Stem cells have two major potentials for therapy; they can repair and replace the damaged tissues and also they can modulate the inflammations. So these therapeutic potentials reserve them as a new promising options for treatment of inflammatory and neurodegenerative disorders. There are some safety results in application of Mesencheymal stem cells(MSCs) in Multiple Sclerosis(MS). some other limited open label trials emphasis to the efficacy of them. Also the results of MESEMS trial, the greatest international randomized clinical trial on application of MSCs in MS, in that we also involved, will be published soon. MSCs in such these trials are autologous and they have been obtained from Bone marrow or Placenta. There is also some promising data of application of autologous hematopoietic stem cells(AHSC) in MS which seems to be promising specially in aggressive MS. In summary, in this review presentation we try to address, the different stem cell types, their mechanism of actions and the promising role of stem cell therapy in MS and as well as some new clinical trials in cell therapy in MS.

\section{4}

\section{Structural Concepts to Target Autoimmune Encephalitis}

\section{Guiscard Friedrich Aldous Seebohm}

Institut für Genetik von Herzerkrankungenm Myozelluläre Elektrophysiologie und Molekularbiologie, Münster, Germany

The Neuroscience Journal of Shefaye Khatam, 2019; 7(3): O14

Autoimmune encephalitis was is associated with autoantibodies targeting NMDA receptors and voltagegated potassium channels. Autoantibodies modulate 
target function which in turn modulates target trafficking. Therefore, autoantibodies effects may include direct functional modification (e.g. calcium influx via NMDA receptors or excitotoxicity by modulation of $\mathrm{Kv}$ channel function). An attractive approach to counteract autoantibody effects is to interfere with autoantibodyassociated conformational changes in target proteins. Structural mechanisms and potential in vitro assays for evaluation of promising compounds will be presented.

\section{5}

\section{Autoimmune Encephalitis}

\section{Jafar Mehwari}

Isfahan University of Medical Sciences, Isfahan, Iran

The Neuroscience Journal of Shefaye Khatam, 2019; 7(3): O15

Autoimmune encephalitis can manifest with several distinct syndromes. The classical presentation of encephalitis consists of a subacute (days to a few weeks) progressive decrease in the level of consciousness, often with fluctuations, and altered cognition. Memory may be impaired early in the clinical course. Patients may progress to coma. While many cases of autoimmune encephalitis are indistinguishable from each other or viral encephalitis, there may be clues to specific autoimmune etiologies. It is typical for patients with autoimmune encephalitis have testing for various infectious etiology. These disorders are strongly cancer associated and involve T-cell responses targeting neurons. The prognosis tends to be poor due to irreversible neuronal killing by these mechanisms. The antibodies in these disorders are useful tumor markers. Antibodies to cell surface antigens, such as the NMDA receptor is common. The associations with cancers are variable, and the prognosis tends to be much better. The serum and CSF titers of NMDA receptor antibodies have been measure. Serum titers did not provide useful information on disease activity. CSF titers were generally higher in patients with acute illness or during relapse compared to patients in remission. Brain MRI in patients with NMDAR, AMPAR, LGI1, Caspr2, and GABA-B antibodies may be normal or show increased T2 signal, especially in the medial temporal lobe.Brain MRI therefore does not distinguish between infectious and autoimmune causes, and a normal brain MRI does not exclude these causes PET or SPECT has shown diverse areas of regional hyper- or hypometabolism in patients with NMDAR, LGI1, Caspr2 or other autoantibodies Seizures may occur at any point during the disease course of anti-NMDAR encephalitis, including at presentation. The extreme delta brush pattern may be observed in patients with anti-NMDAR encephalitis, most often in patients who are comatose. The status epilepticus in the setting of autoimmune encephalitis probably occurs more frequently with NMDAR antibodies overall LGI1 antibodies are associated with (FBD rapid jerking of one side of the face and/or upper extremity). Each seizure tends to be unilateral but they may occur on both sides. Brain biopsy generally is not used in the diagnosis of encephalitis for several reasons. Whenever a paraneoplastic disorder is suspected, tumor screening should be initiated promptl. When a particular autoimmune disease can be confirmed, this will clarify the risk of specific tumors and lead to more focused tumor screening. Most of the synaptic/ cell surface CNS autoantibodies can cause some form of limbic encephalitis, and these diseases can improve with immunotherapy. The criteria for definite autoimmune encephalitis require:

(1) subacute symptoms,

(2) brain MRI abnormalities localized to the medialtemporal lobes

(3) EEG showing epileptic or slow-wave activity originating from the temporal lobes or pleocytosis on lumbar puncture,

(4) exclusion of alterative causes.

AMPA receptor antibodies target the GluR1 orGluR2 subunits of an ionotropic glutamate receptor widely expressed in the brain.

The most common presentation is limbic encephalopathy, but other patients may have a diffuse encephalitis or present first with psychosis LGI1 antibodies are found mostly in older adults (median age of 60 years) and affect more men than women LGI1 antibodies are associated with (FBD rapid jerking of one side of the face and/or upper extremity). Each seizure tends to be unilateral but they may occur on both sides. Treatment of the central nervous system synaptic and cell surface autoimmune disorder: IVIg is widely used as an initial therapy, although its mechanisms in these disease are unclear. Plasma exchange rapidly reduces serum antibodies, but its effectiveness in decreasing CNS antibodies is unknown. Steroids are widely used at high doses initially. In the case of LGI1 antibodies, steroids may be particularly effective, and very slow tapers may be needed. Depletion of developing B cells with rituximab was initially considered a second-line therapy, but is increasingly being used early in the disease course with severely ill patients. Repeated courses of rituximab may be used in some patients, particularly those with relapses. Cyclophosphamide may be useful in patients who are critically ill or refractory to other treatments, but carries risks of infertility and other toxicities. Gonadotropin-releasing hormone (GNRH) agonist treatment should be considered in young women receiving cyclophosphamide to decrease the risk of infertility.

\section{6}

The Role of Neurotropic Factors Derived from Stem Cells in Multiple Sclerosis 


\section{Mohammad Taghi Joghataei ${ }^{1,2}$, Fereshteh Azedi ${ }^{1}$ \\ ${ }^{1}$ Department of Neuroscience, Faculty of Advanced Technologies in Medicine, Iran University of Medical Sciences, Tehran, Iran ${ }^{2}$ Cellular and Molecular Research Center, Iran University of Medical Sciences, Tehran, Iran}

The Neuroscience Journal of Shefaye Khatam, 2019; 7(3): O16

Multiple sclerosis (MS) is a major neurodegenerative disease, which is characterized by the accumulation of abnormal pathogenic proteins due to oxidative stress, mitochondrial dysfunction, impaired autophagy, and pathogens, leading to neurodegeneration and behavioral abnormality. MS is characterized by the presence of lesions or plaques predominantly in the white matter region of the brain and spinal cord. Currently, no cure exists for this disease, and available drugs provide only symptomatic relief. Several studies have highlighted the potential role of cell therapy in the treatment of MS pathology. Clinical trials using stem cells such as mesenchymal stem cell (MSC) in MS patients have shown safety and efficacy. It is well established that MSC transplantation is an approach to regulate the immune system in MS by modulating different signaling pathways and cellular factors. The protective role of neurotrophic factors have emerged in MS research in various surveys. Neurotrophins are a group of structurally related proteins and are able to prevent neural death and improve neural regeneration and remyelination. Notably, stem cells by the release of neurotrophic factors have the ability to protect neuronal population and can efficiently suppress the formation of new lesions in MS patients. Therefore, the future perspectives involve further studies on the insights into mechanisms underlying the cell therapy and neurotherapeutic factors in MS treatment. In this review the role of cell therapy and neurotrphic factors in MS treatment is presented and potential future therapeutic options are discussed.

\section{7}

\section{B Cell Therapy for Multiple Sclerosis}

\section{Morteza Saeidi}

Department of Neurology, Mashhad University of Medical Sciences, Mashhad, Iran

The Neuroscience Journal of Shefaye Khatam, 2019; 7(3): O17

B lymphocytes and plasma cells play an important role in pathogenesis of multiple sclerosis and induce progression of disease by cytokine secretion, antibody production and antigen presentation. B cell directed therapies have very interested in recent years, B lymphocyte cell depletion therapy offers a compelling and promising new option for multiple sclerosis. $\mathrm{B}$ cell activation in multiple sclerosis can induce tumefactive lesions, prominent GAD enhacing lesions, meningeal enhancement, subpial demyelination and... . Monoclonal antibodies targeting CD-20 positive B cells (such as rituximab), can reduce relapses of multiple sclerosis and also delay disability progression of the disease. Ocrelizumab which is a humanized anti CD20 monoclonal antibody, reduces the inflammation in relapsing forms of multiple sclerosis and also reduces disability progression in primary progressive ms. Ocrelizumab, now approved for relapsing and primary progressive multiple sclerosis, differ from rituximab in that it has humanized antibody backbone. Other antiCD20 monoclonal antibodies include ofatumumab (fully humanized with subcutaneous injection), Obinutuzumab (humanized Ig G1) and Ublituximab (an anti-CD20 antibody glycoenginered). Although most disease modifying therapies for MS have traditionally been conceptualized as functioning via $\mathrm{T}$ cell based mechanisms, a growing body of data indicates that all have demonstrable effects on B cells as well, for example beta interferon, glatiramer acetate and fingolimd by shifting B-cell cytokines towards an anti-inflammatory tone. Other mechanism are increasing B-regs, decreasing class II MHC expression and costimulatory molecules on B cells required for $\mathrm{Ag}$ presentstion, sequestering B cells in lymphoid organs.

\section{8}

Effect of Adipose-Derived Mesenchymal Stem Cells on Treatment of Secondary Progressive Multiple Sclerosis Patients

Karim Nikkhah', Fahimeh Lavi', Forouzan Yousefi', Mahmoud Mahmoudi', Hojjat Naderi-Meshkin ${ }^{3}$,Mahdi Mirahmadi', Maryam Rastin ${ }^{2}$, Naser Sanjar Moussavi4, Marjan Erfani ${ }^{1}$

${ }^{1}$ Department of Neurology, Faculty of Medicine, Mashhad University of Medical Sciences, Mashhad, Iran

${ }^{2}$ Immunology Center, BuAli Institute, Department of Immunology Mashhad University of Medical Sciences, Mashhad, Iran

${ }^{3}$ Department of Stem Cell and Regenerative Medicine Research, Iranian Academic Center for Education, Culture and Research (ACECR), Mashhad, Iran

${ }^{4}$ Department of General Surgery, Mashhad Branch, Islamic Azad University, (International Society of Aesthetic Plastic Surgery (ISAPS), Mashhad, Iran

The Neuroscience Journal of Shefaye Khatam, 2019; 7(3): O18

Introduction: Multiple sclerosis (MS) is a chronic neurological disease in CNS which characterized by neuroinflammation and neurodegeneration with aggressive attack of auto-immune cells into myelin sheet axons and causes irreversible disability. Such damages result in gross pathological symptoms in affected young people. Although available strategies slow down MS progression but they aren't effective for the progressive phase of disease. Mesenchymal stem cells are (MSCs) 
self-renewal cells due to their immune-modulatory and neurogenerative traits have achieved fascinating prospects in regenerative medicine. Fat tissue is an optimal source of MSCs because of high abundance and high proliferative rate. Materials and Methods: The study is designed an open label phase $1 / 2$ trial by 10 female (SPMS). Eligible participants are, nonpregnant, unresponsive to other approved treatments, lack of chronic and acute infection or tumor. Patients are predicted to continue to have progression of disease next year. Adipose tissue were removed, after isolation, expand their (AT-MSC) to reach to a range of $5 \times 106$ $\mathrm{MSC} / \mathrm{Kg}$ of body weight. Then infuse their expanded AT-MSC in 2 dosages during 2 injection space 7 days apart. The effect of AT-MSCs on patients were evaluated by assessment of EDSS on baseline, 2 weeks, 1, 3,6 and 9 months after first injection according to MacDonald criteria. Results: In all of patients EDSS was decreased significantly from baseline to 3 months post first injection there after EDSS was increases in 2 patients by 1 point at endpoint of follow up and 6 patients were progression free and in 2 patients EDSS was reduced significantly by 1 point from baseline to 9 months after first injection. Conclusion: MSCs were safe and effective in secondary progressive MS patients and the most pronounced influence was from baseline to 3 months after first MSC administration.

\section{9}

Dietary Patterns with Low Level of Plasma CRactive Protein (hs- CRP) may Reduce Systemic Inflammation and Severity of Multiple Sclerosis Attacks

\section{Ahmad Chitsaz, Zamzam Paknahad', Amir Reza Moravejolahkami}

${ }^{1}$ Isfahan University of Medical Sciences, Isfahan, Iran

${ }^{2}$ Faculty of Nutrition and Food Science, Isfahan University of Medical Sciences, Isfahan, Iran

The Neuroscience Journal of Shefaye Khatam, 2019; 7(3): O19

Dietary patterns seems to be related to clinical manifestations and anthropometric indices in Multiple Sclerosis (MS). Since MS is a chronic inflammatory disorder of central nervous system, so we tried to find related dietary pattern with systemic inflammation, disease severity, relapse rate, severity of fatigue and anthro pometric measurements and quality of life of MS Patients. This cross- sectional study was conducted in Isfahan, Iran, with four subtypes of $261 \mathrm{MS}$ volunteers $(\mathrm{F}=210, \mathrm{M}=51$, mean age 38.98.3). A 168- items food frequency questionnaire (FFQ) serum hs- CRP, multiple sclerosis quality of life- 54 item (MS QOL- 54) questionnaire, extended disability status scale (EDSS), fatigue severity scale (FSS) and visual analog fatigue scale (VAFS) relapse rate, height, weight and Deurenberg
Equation were also used as tools. Data were analyzed by SPSS 24, and using ANOVA, tukey, Chi- sqare and ANCOVA tests. among three identified dietary patterns fruits, vegetables, low fat dairy- based pattern $(n=51)$ and Mediterranean- like pattern $(\mathrm{n}=17)$ were associated to lower serum hs- CRP ( $\mathrm{f}=6.037)$, Padjusted $(<0.01)$, higher quality of life (Padjusted $<0.05$ ), and chronic fatigue $(\mathrm{f}=7.011$, Padjusted $<0.01$ ), lower BMI ( $\mathrm{f}=$ 7.528, Padjusted $<0.01$ ) and percent body fat $(\mathrm{f}=6.135$, Padjusted $<0.01$ ), but no difference was observed about EDSS across the patterns. The most of variables, had significant differences between two mentioned patterns and western- like pattern $(n=194)$. These associations persist when adjusting for age, sex, disease duration and subtypes. adherence to healthy dietary patterns may reduce systemic inflammation, severity of fatigue, MS attacks, improve quality of life and normalize weight especially body fat in MS patients.

\section{$\mathbf{O 2 0}$}

\section{Diagnosis and Treatment of Myasthenia Gravis}

\section{Fariba Zemorshedi}

Department of Neurology, Faculty of Medicine, Mashhad University of Medical Science, Mashhad, Iran

The Neuroscience Journal of Shefaye Khatam, 2019; 7(3): O20

myasthenia gravis is the most common acquired disorder of neuromuscular transmission. Due to the production of pathogenic autoantibodies that bind to components of the neuromuscular junction, the most common being the Anti-Achetyl coline receptor. myasthenia gravis produces symptomatic weakness that predominates in certain muscle groups and typically fluctuates in response to effort and rest. Diagnosis of myasthenia gravis is based on history and physical exam, pharmacological tests, serological (immunological) tests and electrophysiological tests. Treatment options include symptomatic therapy, intravenous immunoglobuline (IVIG)/plasmapheresis, thymectomy, corticosteroids, immunosuppressive agents, monoclonal antibodies and stem cell transplantation. Factors to be considered for treatment are age of the patient, severity of disease, medical comorbidities, side effect profile of the agent selected, monitoring requirements, speed of the onset of action and cost of drug.

\section{1}

\section{Necrotizing Autoimmune Myopathy}

\section{Shahriar Nafissi}

Department of Neurology, Tehran University of Medical Sciences, Tehran, Iran

The Neuroscience Journal of Shefaye Khatam, 2019; 7(3): O21

Necrotizing autoimmune myopathy (NAM) is a type of 
immune-mediated myopathy characterized by myofiber necrosis but with no or minimal inflammatory cell infiltrate on muscle biopsy and specific antibodies. Anti-signal recognition particle (SRP) and antihydroxy-3-methylglutaryl-CoA reductase (HMGCR) autoantibodies are closely associated with NAM and define unique subtypes of patients. Importantly, the new European Neuromuscular Centre criteria recognize antiSRP myopathy, anti-HMGCR myopathy, and autoantibody-negative NAM as three distinct subtypes of NAM. Anti-SRP myopathy patients have more severe muscle involvement, with a better response to immunosuppressive regimens that include rituximab. In contrast, antiHMGCR myopathy is often associated with statin exposure with a good response to IVIG, even as monotherapy. Both anti-SRP and anti-HMGCR myopathy tend to be most severe in younger patients. Furthermore, children with these forms of NAM may present with dystrophy-like features which are potentially reversible with immunosuppressant treatment. Some patients with NAM develop severe weakness soon associated with fatty replacement of muscle, suggesting that intense and early immunosuppressant therapy may provide the best chance to avoid long-term disability.

\section{$\mathbf{0 2 2}$}

\section{MOG Antibody Disease}

\section{Mohammad Hossein Harirchian}

Iranian Center for Neurological Research, Tehran University of Medical Sciences, Tehran, Iran

The Neuroscience Journal of Shefaye Khatam, 2019; 7(3): O22

Myelin Oligodendrocyte Glycoprotein (MOG) is a glycoprotein expressed selectively at the outermost surface of myelin sheaths and oligodendrocyte membrane. It is found exclusively in the CNS to serve as a necessary "adhesion molecule" to provide structural integrity to the myelin sheath. This glycoprotein has a role in different disease such as multiple sclerosis (MS) and adrenoleukodystrophy. MOG antibody is involved in a clinical spectrum of disease named as MOG antibody disease (MOG-AD). These include neuromyelitis optica spectrum disorder (NMOSD), acute demyelinating encephalomyelitis (ADEM), focal cortical inflammatory disease, and some conditions such as NMD receptor encephalitis. An ADEM-like presentation is more common in children. MOG antibodies are found in over half of these patients and in virtually all patients with multiphasic ADEM. Persistence of MOG antibodies in children with ADEM appears to predict further relapses, particularly of optic neuritis or ADEM. MOG antibody positive ADEM patients do not differ significantly from MOG antibody-negative patients in terms of clinical presentation or demographics. Focal cerebral cortical inflammatory disease has been recently reported in benign, unilateral, cerebral cortical encephalitis with epilepsy in adult patients with steroid-responsive encephalitis. MOG-AD could be presented with MS/ NMOSD like features. Thirty three percent of adult patients with MOG-AD meet McDonald's criteria for MS, but it should be considered that some drugs approved for MS might be ineffective or even harmful in MOG-EM. Isolated optic neuritis is the most common onset presentation $(55 \%-64 \%)$ which typically involves the anterior segment (papillitis). Transverse myelitis in MOG-AD typically presents as longitudinal extensive Transverse myelitis (LETM). A LETM lesion in MOG$\mathrm{AD}$ disease seen on MR scan of the spinal cord tends to be central and associated with swelling in the acute phase. It involves the conus more often than in the AQP4 antibody LETM. MOG antibody disease can be either monophasic or relapsing. Attacks generally recover, but some patients are left with residual disability: mostly sphincter, erectile or visual. MS-typical brain lesions on MRI are very unusual in MOG antibody or AQP4 antibody disease, including Dawson's fingers, inferior temporal lobe lesions and S-shaped/curved juxtacortical lesions. In CSF Around half of the patients have elevated white cell counts, and in 5\%-10\% the lymphocyte count is as high as 100-300 cells/ $\mu \mathrm{L}$. Similarly around half have an elevated CSF protein concentration, which can be above $100 \mathrm{mg} / \mathrm{dL}$ in $10 \%$. Oligoclonal bands restricted to the CSF occur in up to $15 \%$ of patients. Cell based indirect fluorescence test and employing full length human MOG as the target antigen MOG IgG is necessary for diagnosis.

Recommended indications for MOG-IgG testing are:

1. Monophasic or relapsing acute optic neuritis, myelitis, brainstem encephalitis, encephalitis, or any combination.

2. Radiological or, only in patients with a history of optic neuritis, electrophysiological (VEP) findings compatible with CNS demyelination.

3. At least one of the following findings including simultaneous bilateral acute $\mathrm{ON}$, prominent papilledema/ papillitis/optic disc swelling during acute $\mathrm{ON}$, unusually high $\mathrm{ON}$ frequency, particularly severe visual deficit/ blindness, particularly severe or frequent episodes of acute myelitis or brainstem encephalitis, permanent sphincter and/or erectile disorder after myelitis, patients diagnosed with "ADEM", "multiphasic ADEM", acute respiratory insufficiency, disturbance of consciousness, behavioral changes, or epileptic seizures (radiological signs of demyelination required), disease started within 4 days to 4 weeks after vaccination, otherwise unexplained intractable nausea and vomiting or intractable hiccups, co-existing teratoma or NMDAR encephalitis. Management is largely adopted from NMOSD. Acute relapses are usually treated immediately With Methylprednisolone, plasma exchange or intravenous 
immunoglobulin. MOG-EM is associated with a high risk of flare-ups after cessation of steroid treatment for acute attack. In patients with persistent antibody treatment should be continued for up to 12 months. In relapsing disease a corticosteroid-sparing agent such as Azathioprine, Methotrexate, Mycophenolate mofetil, or Rituximab could be added.

\section{3}

The Potassium Channel KCNK2 is a Regulator of Immune Cell Trafficking and Inflammatory Responses in Idiopathic Inflammatory Myopathies

\section{Thomas Müntefering, Alexander P.E. Michels, Sven} G. Meuth, Tobias Ruck

Department of Neurology, University of Münster, Münster, Germany

The Neuroscience Journal of Shefaye Khatam, 2019; 7(3): O23

KCNK2, a two-pore-domain potassium channel, has been implicated as important regulator of leukocyte transmigration into the central nervous system. KCNK2-/- mice showed an increased disease severity in experimental autoimmune encephalomyelitis (animal model of multiple sclerosis). Likewise, an immune-cell infiltration can be observed as pathogenic hallmark in idiopathic-inflammatory-myopathies (IIM) as well. Therefore, we investigate if KCNK2 is involved in peripheral autoimmune responses of IIMs. In accordance, we found KCNK2 expression in primary murine microvascular endothelial cells (PMMEC) and in primary murine muscle cells (PMMC) on gene and protein level. Stimulation with pro-inflammatory cytokines led to a downregulation of KCNK2 expression on differentiated PMMC and PMMEC. Interestingly, KCNK2-/- or pharmacological blockade of KCNK2 with spadin increased the expression of pro-inflammatory activation markers and adhesion molecules of these cells. To further elucidate the regulatory role of $\mathrm{KCNK} 2$, we found significantly more adhered $\mathrm{T}$ cells on KCNK2-/- or spadin treated PMMEC in vitro under low shear flow and in vivo in microvessels of the cremaster muscle using intravital imaging. In summary, KCNK2 might be critically involved in the regulation of immunological processes, especially immune-cell trafficking of IIMs and might provide a deeper understanding of the regulatory mechanisms to identify new, urgently needed, therapeutic targets.

\section{O24}

\section{Movement Disordes Emergency}

\section{Ebrahim Poorakbar}

Mashhad University of Medical Sciences, Mashhad, Iran

\section{The Neuroscience Journal of Shefaye Khatam, 2019; 7(3): O24}

Many acute potentially life-threatening medical conditions have hyperkinetic or hypokinetic movement disorders as their hallmark. Emergent movement disorders are which develope over hours or several days and morbidity and mortality can result from failure to appropriately diagnose and manage the patient. Various movement disorders includ acute parkinsonism, neuroleptic malignant syndrome, respiratory compromise in MSA, dystonic storm, oculogyric crisis and hemiballism, parkinson hyperpyrexia syndrome and psychosis. I will explain these topics in this presentation.

\section{5}

\section{Immunotherapy in CVA}

\section{Reza Rikhtegar, Sanam Dolati}

Departments of Neurology, Faculty of Medicine, Tabriz University of Medical Sciences, Tabriz, Iran

The Neuroscience Journal of Shefaye Khatam, 2019; 7(3): O25

Abstract Introduction: Immune inflammation mediated by $\mathrm{T}$ cells that interact with microglia leads to brain damage after ischemic stroke (IS). CD4+CD25+ T (Treg) cells and Th17 cells play important roles in immunopathogenesis of IS. Method and material: Thirty patients with IS and 30 individuals as healthy control group were enrolled in this study. In the present study, the frequency of Th17 and Treg cells, the expression levels of related transcription factors, and the secretion levels of associated cytokines were assessed by Flow cytometry, Real-time PCR, and ELISA, respectively, at 1,5 , and 10 days after stroke as compared with healthy control subjects. Results: A significant reduction in frequency of Treg cells was observed in patients with IS at 1 day $(p<0.0001)$ and 5 days $(p=0.016)$ after stroke, but not 10 days. The expression levels of FOXP3 were decreased significantly at 1 day $(\mathrm{p}=0.0005)$ and 5 days $(p=0.007)$ after stroke in compared with the control group. Also TGF- $\beta$ secretion levels were decreased at 1 day $(\mathrm{p}=0.001), 5$ days $(\mathrm{p}=0.002)$, after stroke in patients with IS in compared with the control group. We showed that the number of Th17 cells and expression levels of ROR $\gamma t$ were significantly increased in patients with IS at 1 day and 5 days after stroke $(p<0.0001)$, but not in 10 days after stroke. IL-17 secretion levels were increased significantly at 1 day $(\mathrm{p}=0.0003), 5$ days $(\mathrm{p}=0.005)$, and 10 days $(p=0.005)$ after stroke in compared with the healthy control group. Conclusion: Our results recommend that a numeral and functional imbalance between Th17 and Treg cells occurs in patients with ischemic stroke. Manipulating the balance between the anti- and pro-inflammatory properties of $\mathrm{T}$ cells may offer a hopeful therapeutic approach to speed up the recovery from IS. 


\section{6}

Movement Disorders with Neuronal Autoantibodies

\section{Nahid Olfati}

Department of Neurology, Faculty of Medicine, Mashhad University of Medical Sciences, Mashhad, Iran

The Neuroscience Journal of Shefaye Khatam, 2019; 7(3): O26

An increasing number of autoantibody-associated neurological disorders are recognized in the recent years which abnormal movements constitute a part or dominate their clinical picture. In comparison to the classic movement disorders that have a neurodegenerative pathologic basis, movement disorders associated with autoantibodies (AAMDs) are more important to be diagnosed correctly since they are generally responsive to immunotherapeutic measures. AAMDs can present with various syndromes in the abnormal movements spectrum including parkinsonism, dystonia, myoclonus, chorea, ataxia, tremor, and sleep behavior disorders, among others. These disorders could be classified based on their clinical presentations or based on the type of structures that serve as target for autoantibodies. Targets are located either on the cell surface or intracellularly, in synaptic cytoplasm, cytosol, or the nucleus. Proper testing of the disease markers will allow timely diagnosis and treatment which include various immunosuppressive and immunomodulatory as well as a number of symptomatic treatment approaches.

\section{$\mathbf{0 2 7}$}

\section{Inflammatory Pathways in Parkinson's Disease}

\section{Mehri Salari}

Shahid Beheshi University of Medical Sciences, Tehran, Iran

The Neuroscience Journal of Shefaye Khatam, 2019; 7(3): O27

The etiology of Parkinson's disease (PD) is yet to be fully understood but it is becoming more and more evident that neuronal cell death may be multifactorial in essence. The main focus of PD research is to better understand substantia nigra homeostasis disruption, particularly in relation to the wide-spread deposition of the aberrant protein $\alpha$-synuclein. Microarray technology contributed towards PD research with several studies to date and one gene, ALDH1A1 (Aldehyde dehydrogenase 1 family, member A1), consistently reappeared across studies including the present study, highlighting dopamine (DA) metabolism dysfunction resulting in oxidative stress and most probably leading to neuronal cell death. Neuronal cell death leads to increased inflammation through the activation of astrocytes and microglia. Using our dataset, we aimed to isolate some of these pathways so to offer potential novel neuroprotective therapeutic avenues. To that effect our study has focused on the upregulation of $\mathrm{P} 2 \mathrm{X} 7$ (purinergic receptor P2X, ligand- gated ion channel, 7) receptor pathway (microglial activation) and on the NOS3 (nitric oxide synthase 3) pathway (angiogenesis). In summary, although the exact initiator of striatal DA neuronal cell death remains to be determined, based on our analysis, this event does not remain without consequence. Extracellular ATP and reactive astrocytes appear to be responsible for the activation of microglia which in turn release proinflammatory cytokines contributing further to the parkinsonian condition. In addition to tackling oxidative stress pathways we also suggest to reduce microglial and endothelial activation to support neuronal outgrowth.

\section{$\mathbf{0 2 8}$}

\section{BBB in Inflammatory CNS Disorders}

\section{Masoud Etemadifar}

Isfahan University of Medical Sciences, Isfahan, Iran

The Neuroscience Journal of Shefaye Khatam, 2019; 7(3): O28

Multiple sclerosis(MS) is an inflammatory disorder of the central nervous system with an increasing rate of prevalence in IRAN during last decade.MS clearly results from the blood brain barrier(BBB) breakage and the resulting cellular damage and inflammatory process. BBB disruption and vascular changes play an important role in MS pathogenesis. Vascular pathology in a background of genetically predisposed individual, influenced by several environmental factors such as pathogens, Vit D deficiency, obesity and smoking, may be a critical initiator of a series of events including hypoxia, protein deposition and immune cell egress that allows the development of a CNS-specific immune response and combination of inflammation and degeneration two types of inflammatory process occur in MS, which develop in parallel but partially independent from each other. The first of them is invasion of T- and B-lymphocytes with profound blood brain barrier leakage, which predominately affects the white matter, and which gives rise to classical active plaques. The second type of inflammation is a slow accumulation of T-cells and B-cells in the absence of major blood brain barrier damage in the connective tissue spaces of the brain, such as the meninges and the large perivascular Virchow Robin spaces, where they form structures in part resembling tertiary lymph follicles. in this type of inflammation B cells have important role and inflammation lead to formation of subapical demyelinated lesions in the cerebral cortex, with slow expansion of pre-existing lesions in the white matter and with diffuse neurodegeneration in the normal appearing white or gray matter. The first type of inflammation dominates in acute and relapsing MS. The second type of inflammation is already present in early stages of MS, but gradually increases with disease duration and patient age in progresive types of 
MS.Presence or absence of inflammationis a key point in choosing drugs and monitoring the treatment response. MRI represents the most sensitive non-invasive tool to monitor inflammation in the clinical practice.In the early phase of inflammation MRI also detects new lesions as extrusion of gadolinium contrast agents across the BBB. according to MC Donald criteria 2017 The occurrence of MRI lesions is used to confirm diagnosis and has been validated as surrogate marker of relapse to monitor response to treatments. New imaging techniques have been developed to study diffuse inflammation taking place outside the focal plaques.

\section{$\mathbf{O 2 9}$}

(Auto-) Immune Epilepsy: Mechanisms of Inflammatory Epileptogenic Neurodegeneration

\author{
Nico Melzer \\ Münster, Germany \\ The Neuroscience Journal of Shefaye Khatam, 2019; 7(3): O29
}

(Auto-) immune inflammation predominantly affecting the allocortex of the amygdala and hippocampus as well as the neocortex recently emerged as a major cause of mesial temporal lobe epilepsy and epileptic encephalopathy. In both major parts of the cortex, neurons are assumed to be targets of adaptive B cell and $\mathrm{T}$ cell responses causing aberrant neuronal excitability together with variable neurodegenerative effects promoting the emergence of seizures and epilepsy together with a variety of neuropsychiatric symptoms. In my talk, I will give an overview on the current concept of immune-mediated epileptogenic neurodegeneration.

\section{O30}

\section{Immune-Mediated Cerebellar Ataxias}

\section{Mehran Ghaffari}

Shahid Beheshti University of Medical Sciences, Tehran, Iran

The Neuroscience Journal of Shefaye Khatam, 2019; 7(3): O30

The current article reviews the clinical presentations of immune-mediated cerebellar ataxia and analyzes the association with autoantibodies. Emerging evidence suggests that autoimmunity is involved in a significant proportion of patients presented with ataxia. Moreover, numerous autoantibodies have recently been described in association with cerebellar ataxias. The cerebellum is a vulnerable target of autoimmunity in the CNS and immune-mediated cerebellar ataxia is an important differential diagnosis in patients presenting with signs and symptoms of cerebellar disease. Immune-mediated cerebellar ataxias include gluten ataxia, paraneoplastic cerebellar degeneration, GAD antibody associated cerebellar ataxia, and Hashimoto's encephalopathy among others. More than 30 different autoantibodies targeting brain antigens have been reported in patients with Immune-mediated cerebellar ataxias, many of which are of paraneoplastic origin. Efficient biomarkers are still lacking and in many cases the diagnosis has to rely on a body of converging evidence.

\section{1}

\section{Approach in Optic Neuropathy}

\section{Alireza Nikseresht}

Shiraz University of Medical Sciences, Shiraz, Iran

The Neuroscience Journal of Shefaye Khatam, 2019; 7(3): O31

In this review we want to overview all typical optic neuropathy and their characteristic and specially we want to discuss important clinical and imaging red flag for optic neuritis for neurologist to differentiat them from atypical optic neuritis.Because different causes of optic neuropathy have different treatment and diagnostic approach. For example, sever vison loss can be due NMOSD and its treatment can be responsive to plasmaexchang than corticosteroid alone. we must review red flag for typical optic neuritis before considering of treatment and diagnosis.

\section{$\mathbf{O 3 2}$}

Update Dementia: Diagnostics, Therapeutic Options - and a Glimpse into the Future

\section{Thomas Duning}

Department of Neurology, University of Münster, Münster, Germany

The Neuroscience Journal of Shefaye Khatam, 2019; 7(3): O32

Due to demographic trends worldwide, the number of dementia patients will increase dramatically in the coming years. Serious estimates assume that the number of patients will double by about 2033. In order to be able to treat the disease effectively, a meaningful and targeted diagnosis that reveals the etiology of dementia symptoms is crucial. However, the diagnosis of dementia can be challanging. In our lecture, we show which diagnostic strategies can be used to differentiate primary neurodegenerative diseases. Beside imaging methods, the focus will be on CSF analysis and, above all, on clinical symptoms and examination strategies. In addition to Alzheimer's pathology, we show typical symptoms of the most important differential diagnoses, i.a. frontotemporal lobar degenerations, Lewy body dementia, vascular dementia, or dementia due to Parkinson's disease. Additionally, we will highlight secondary degenerative processes. We will discuss the currently available and guideline-appropiate therapeutic options and give an outlook on future diagnostic and 
therapeutic strategies for dementia, in particular for Alzheimer's pathology.

\section{$\mathbf{O 3 3}$}

Neuro Inflammation in Migraine, From Pathogenesis to Treatment

\section{Mansoureh Togha, Zeinab Ghorbani}

Department of Neurology, Iranian Center of Neurological Research, Neuroscience Institute, Tehran University of Medical Sciences, Tehran, Iran

The Neuroscience Journal of Shefaye Khatam, 2019; 7(3): O33

Migraine has been known as the first cause of disability in under 50 s and affects approximately $11 \%$ of the adults in the world. Emerging evidence has focused on "neurogenic inflammation" theory in migraine pathogenesis which involves the release of pro-inflammatory substances that lead to activation and sensitization of peripheral nociceptors. The sensory fibers to the intracranial vasculature have their origin in the trigeminal ganglion that store calcitonin gene-related peptide (CGRP), amylin, substance P (SP), Nitric oxide (NO), neurokinin $\mathrm{A}$ and $\mathrm{B}$, pituitary adenylate cyclase-activating peptide (PACAP), vasoactive intestinal peptide (VIP), dynorphine, and nociception. Released peptides such as CGRP binds to the CGRP receptor on the smooth muscle cells causing a vasodilatory response with increase in the meningeal blood flow in the dural vasculature. Therefore, the augmentation of neurotransmitters CGRP, SP, PACAP, and VIP which activates and provokes mast cells release, interacts with blood vessels, neurons, and immune cells, generating inflammation by provoking vasodilation, extravasation, and glial cell activation with the production of pro-inflammatory IL-1 family members: All these activities can trigger headache. Further, there are several studies reporting augmented plasma pro-inflammatory factors including CRP, ILs e.g. IL- 1 and IL- 6 and TNF- $\alpha$ in migraineurs. Also in different studies increased levels of NO, CGRP, PACAP, TNF- $\alpha$ and IL- $1 \beta$ were observed in the CSF and plasma samples of migraineurs. NO, CGRP and PACAP are among the most studied factors in migraine pathogenesis and recent studies have emphasized on new antimigraine drugs that could combat inflammatory state. These drugs certainly interfere with signaling pathways of CGRP, a vasodilatory neuropeptide expressed in the cranial sensory nerves and PACAP, a parasympathetic vasodilator peptide that is linked to cranial autonomic symptoms in migraine. To date, 4 monoclonal antibodies have been developed for migraine prophylaxis that act on the CGRP pathway. Erenumab, fremanezumab and galcanezumab were approved by the FDA for migraine prevention. There is also ongoing development of therapeutic approaches targeting both PACAP and the PAC1 receptor with a current emphasize on PACAP- related antibodies development. Although preliminary data propose that targeting PAC1 receptors might be most effective, it is also suggested targeting VPAC1 and VPAC2 receptors may still be of clinical benefit.

\section{4}

The Role of Different Diets in Migraine Control with an Emphasize on Suppressing Neuroinflammation

\section{Soodeh Razeghi Jahromi, ${ }^{1,2}$ Zeinab Ghorbani ${ }^{2}$}

${ }^{1}$ Department of Clinical Nutrition and Dietetics, Faculty of Nutrition and Food Technology, Shahid Beheshti University of Medical Sciences, Tehran, Iran

${ }^{2}$ Department of Headache, Iranian Center of Neurological Research, Neuroscience Institute, Tehran University of Medical Sciences, Tehran, Iran

The Neuroscience Journal of Shefaye Khatam, 2019; 7(3): O34

Migraine headache has been ranked as the highest contributor to disability in under 50 year's old population in the world. Extensive researches have been conducted in order to clarify the pathological mechanisms of migraine. Although much uncertainty remains, the presence of neuroinflammation and its consequent stimulation of the trigeminal vascular system might be principally responsible for migraine pain. However, the advances in the study of mechanisms of migraine should be integrated into treatment approaches for providing pain relieving effects and increasing quality of life in patients with migraine. With respect to this, one of the interesting areas within the integrative health studies pertains to dietary interventions. Although the number of studies concerning effects of diet on migraine is not yet very large, the current article will review the available evidence in this area. So far, the first category of diets that their effect has been studied in relation to migraine includes fasting and ketogenic diets, low-calorie diet, and modified Atkins diet. These diets could mainly act through compensating serotoninergic dysfunction, inhibition of neuronal excitability, decreasing CGRP levels, brain mitochondrial function improvement, suppressing neuroinflammation and thus could reduce the severity of migraine headache. Also, there is a reasonable relationship between obesity and migraine. Therefore, weight loss diets have been reported to be effective in migraine improvement. Suppression of neuroinflammation, regulating adiponectin and leptin levels, affecting on hypothalamic function, enhancing insulin secretion and improvement of depression, a comorbidity of both obesity and headache, are among the proposed mechanisms of action of weight loss diets in migraine enhancement. Further, dietary fat intake and migraine headache have been linked to each other. The balance between the omega- 6 and omega- 3 fatty acids, contribute to inflammatory control in response to the environmental metabolic changes. Moreover, 
dietary fats affect serotonin release from platelet. On the flip side, evidence showed the worsening of preexisting migraine in idiopathic thrombocytopenia, mainly during platelet destruction phase. Suppressing platelet aggregation seems to have therapeutic value in migraine prevention. Therefore, any modalities like reducing and plasma free fatty acids and plasma lipids that can enhance platelet aggregation, seems to decrease the frequency and duration of migraine headache. In addition, it has been proposed that elemental diet could be effective in migraine control. The suggested mechanism could be relevant is that in both migraine and food sensitivities, inflammation induced by food could make the pro-inflammatory environment which is needed for the induction of headache by other triggers. And finally, low glycemic diet might also improve migraine characteristics through inhibition of neuroinflammation and correction of oxidative stress.

\section{5}

\section{Neurogenic Inflammation in Migraine: New Horizons in Migraine Treatment}

\author{
Nooshin Yamani, ${ }^{1,2}$ \\ ${ }^{1}$ Department of Neurology, Faculty of Medicine, Zanjan \\ University of Medical Sciences, Zanjan, Iran \\ ${ }^{2}$ Department of Headache, Iranian Center of Neurological \\ Research, Neuroscience Institute, Tehran University of Medical \\ Sciences, Tehran, Iran
}

The Neuroscience Journal of Shefaye Khatam, 2019; 7(3): O35

Migraine is a common primary headache disorder and ranked as the second most disabling disorder worldwide. Although various studies performed to understand the pathogenesis of migraine disorder, the exact pathogenic mechanism is still unclear. Recently, considerable improvement in molecular biology and pharmacology illustrated that neurogenic inflammation is likely to be a mechanism involved in the migraine pathophysiology. This neurogenic inflammation is described by the release of sensory neuropeptides such as Calcitonin gene-related peptide (CGRP) from activated trigeminal neurons, leading to vasodilation, degranulation of mast cells, and sensory transmission. Further research advancements considering the role of neurogenic inflammation in migraine, proposed that targeting CGRP pathway by blocking the CGRP ligand or CGRP receptor might have putative role in the treatment of migraine. Targeting the CGRP pathway led to development of novel therapeutic anti-migraine drugs: CGRP receptor antagonists (Gepants) for acute treatment of migraine and anti CGRP monoclonal antibodies as promising preventive treatments. I will review the CGRP peptide and its role in migraine pathophysiology in order to better understand the mechanism through which, emerging novel therapies targeting the CGRP pathway act. I will also summarize the main results of phase II/III randomized clinical trials on these drugs.

\section{6}

\section{Human Glioblastoma Stem Cells and Nano- Curcumin}

\section{Sajad Sahab Negah, Fatemeh Ariakia, Mohammad Jalili-Nik}

Neuroscience Research Center, Mashhad University of Medical Sciences, Mashhad, Iran

The Neuroscience Journal of Shefaye Khatam, 2019; 7(3): O36

Glioblastoma multiform (GBM) is a progressive brain tumor that causes mortality around the word. Current treatment is not successful because drug delivery is so poor. Recent evidences have shown that nanoformulating of drugs could be useful for treatment of GBM. Among different types of nano-particles, niosomes are biodegradable, biocompatible, and nonimmunogenic structure. On the other hand, today's researchers have focused on natural components as an antitumor agents. Curcumin is introduced as a key and functional form of natural component in cancer biology. In the present study, we improved delivery of curcumin in the shell of niosome as a nano-carrier. To evaluate the anti-tumor effects of nano-curcumin, we treated glioblastoma stem cells (GSCs) with nano-curcumin. Our results showed that nanno-curcumin decreased proliferation, migration, and invasion of GSCs. Furthermore, we showed that the increase of ROS generation and apoptosis in GSCs when treated with nano-curcumin. To summarize, nanocurcumin is more efficient than free curcumin and can be proposed for GBM treatment.

\section{Poster Presentations}

\section{P1}

\section{Regulatory Function of Cytokines Signaling in Idiopathic Inflammatory Myopathies}

\section{Sina Helms}

Medical Education Friedrich-Alexander-University ErlangenNuernberg, Department of Neurology, University Hospital Muenster, Muenster, Germany

The Neuroscience Journal of Shefaye Khatam, 2019; 7(3): P1

The complexity of intercellular interplay in inflammatory and autoimmune processes is mainly regulated by cytokines, chemokines and other soluble and membrane-bound mediators produced by a variety of cells. We established the isolation and cultivation of functional primary murine skeletal muscle cells and primary murine microvascular cells to characterize the regulatory function of cytokines signalling in idiopathic 
inflammatory myopathies, focusing on the effect on GM-CSF and its receptor. In order to achieve this, we stimulated the cells with TNF, IF and a combination of the two. These experiments demonstrated a functional expression of GM-CSF and its receptor in both cell types. The highest expression levels of GM-CSF were observed under treatment with TNF and TNF+IF by primary murine skeletal muscle cells. Under basal conditions, the cells showed no detectable GM-CSF expression. Interestingly, muscle cells expressed higher levels of GM-CSF receptor, while endothelial cells expressed higher levels of GM-CSF, also under basal conditions. The stimulation with IFN of primary murine microvascular endothelial cells led to a significant downregulation of GM-CSF receptor expression below detection limits.

\section{P2}

Histological and Functional Characterization of Connexin32 in A Model of Experimental Autoimmune Encephalitis

\section{J. Vogt, M. Cerina, P. Hundehege, A. M. Herrmann, K. Göbel, T. Budde, S.G. Meuth}

The Neuroscience Journal of Shefaye Khatam, 2019; 7(3): P2

Introduction: Multiple Sclerosis (MS) is a chronic inflammatory and neurodegenerative disease manifesting itself in de- and remyelination in the central nervous system (CNS) leading to consequent neuronal hyperexcitability and functionality damage. Recent studies found that loss of the gap-junction protein $\mathrm{Cx} 32$ is associated with occurrence of demyelinating disorders, downregulation of the GABAergic system and therefore hyperexcitability. Materials and Methods: Based on that we aimed to clarify the role of $\mathrm{Cx} 32$ in driving the pathophysiological process in MS. In order to achieve our goal, we initially performed immunohistochemistry to characterize the cytomorphology of the CNS of transgenic mice lacking $\mathrm{Cx} 32$ (KO). Results: Our results inn dicated that in $\mathrm{Cx} 32-\mathrm{KO}$ the content of myelin is $32 \%$ lower in comparison to control littermates (WT, $n=3$; $\mathrm{p}<0.0001$ ). After inducing experimental autoimmune encephalitis (EAE) in mice by immunization with the myelin protein MOG35-55, Cx32-KO showed a significantly more severe phenotype in comparison to Cx32WT approximately 15 days after immunization $(4.9 \pm$ 0.3 vs. $3.4 \pm 0.2$, respectively; $n=10 ; p<0.001$ ), when the disease is mainly characterized by neurodegeneration. Cx32-WT start recovering and remyelination while the Cx32-KO show a worsening of their clinical score. Conclusion:Taken together, our results suggest that a lack of Cx32 modifies the outcome of EAE by enhancing the effects of demyelination and impairing remyelinating abilities, thereby leading to increased neuronal death and more severe symptoms.
P3

Activation of Platelets $\quad-\quad$ a Roadway to
Neuroinflam mation

Anna Vogelsang, Susann Eichler and Sven G. Meuth

Department of Neurology with Institute of Translational Neurology, University of Muenster, Germany

The Neuroscience Journal of Shefaye Khatam, 2019; 7(3): P3

Introduction: Blood-brain-barrier disruption and immune cell infiltration are hallmarks of multiple sclerosis (MS) and experimental autoimmune encephalomyelitis (EAE). Not only immune cells, but also platelets infiltrate the central nervous system (CNS) and accumulate in lesions during neuroinflammation. Inside lesions, degranulating platelets secrete inflammatory and vasoactive substances, which seem to promote immune cell infiltration. Recent studies demonstrated that depletion of platelets during the effector phase of EAE diminish gene expressions of intercellular-adhesion-molecule-1 (ICAM-1) and vascular-cell-adhesion-molecule-1 (VCAM-1) in CNS tissue 1. However, a deeper understanding of the interplay between platelets and endothelial cells is required. Materials and Methods: Here, we examined the impact of platelet-derived releasate on brain microvascular endothelial cells. Therefore, platelets were either activated by thrombin or adenosine diphosphate (ADP). Then, primary endothelial cells treated with plateletderived releasates were analyzed for gene expressions of cell-adhesion and tight-junction molecules. Results: Platelet-derived releasate generated with ADP leads on endothelial cells to a pronounced upregulation of ICAM1 (39.18-fold) and VCAM-1 (5.8-fold) that support diapedesis of immune cells. Whereas the stimulation with thrombin mediates opposite effects. Conclusion: We conclude that different activation pathways seem to alter the proinflammatory activity of platelets. However, further investigations are needed to elucidate the underlying mechanisms of platelet activation and its impact on neuroinflammation.

\section{P4}

Inhibition of the Platelet-Receptor P2Y12 Moderates Neuroinflammation

Lara-Maria Preuth, Susann Eichler, Niklas Huntemann, Anna Vogelsang, Sven G. Meuth

Department of Neurology with Institute of Translational Neurology, University of Muenster, Muenster, Germany

The Neuroscience Journal of Shefaye Khatam, 2019; 7(3): P4

Introduction: Patients suffering from multiple sclerosis (MS) have an augmented risk for cardiovascular diseases 
associated with abnormal platelet functions. However, platelet-mediated contributions to inflammatory disorders of the central nervous system (CNS), such as experimental autoimmune encephalomyelitis (EAE), a model to study $\mathrm{MS}$, remain poorly understood. In EAE, platelets were found accumulating in the CNS parenchyma, thereby amplifying the (neuro) inflammatory response. Materials and Methods: Here, we show that pharmacologic blockade of platelet-receptor P2Y12 with elinogrel renders mice less susceptible to EAE. Results: While disease onset was unaltered, elinogrel treatment was associated with a reduced $(\mathrm{p}<0.03)$ disease maximum and also with reduced prevalence of inflammatory infiltrates compared to the control group $(5,1 \pm 1,4 \%$ versus $9,6 \pm$ $1,2 \%, n=6, p<0.05)$. Amelioration was accompanied by reduced numbers of interleukin-17A-producing T-helper cells as determined via intracellular cytokine staining at the disease peak (day 15). Further, an increase in CD8+ and $\mathrm{CD} 8+\mathrm{CD} 11 \mathrm{c}+$ cells, but no significant difference in macrophages, microglia, B-cells and plasmatic dendritic cells of elinogrel-treated mice was found. Conclusion: Overall, our findings show that inhibition of platelet activation is beneficial in an animal model of MS and thus indicate in addition to the "classical" immunological-oriented pathophysiological concepts an alternative strategy for the treatment of MS.

\section{P5}

\section{Investigating the Role of the Mitochondrial Calcium} Uniporter in Inflammatory Neurodegeneration

\section{Laura Bierhansl, Petra Hundehege, Iska Loesmann, Petra Henrich-Noack, Sven G. Meuth}

Clinic for Neurology with Institute of Translational Neurology, University Clinic Muenster, Muenster, Germany

The Neuroscience Journal of Shefaye Khatam, 2019; 7(3): P5

Introduction: The "Mitochondrial Calcium Uniporter" (MCU) regulates $\mathrm{Ca} 2+$-influx into mitochondria. In case of neurological disease, an excessive $\mathrm{Ca} 2+$ influx (Caoverload) plays a major role in post-lesional neuronal degeneration and death. Materials and Methods: To further elucidate the role of the MCU in CNS pathophysiology, we generated neuron-specific MCU knockout mice in order to study them in experimental autoimmune encephalomyelitis (EAE), a mouse model of multiple sclerosis (MS). We hypothesize that neuronal damage can be reduced by inactivating the MCU, offering therapeutic opportunities. We crossbred neuron-specific cre (NexCre) transgenic and homozygous loxP floxed mice. After one generation we obtained hemizygous, neuron-specific Cre (NexCre) mice which were heterozygous for the floxed MCU gene. These animals were crossbred with homozygous, loxP floxed MCU gene mice to obtain an F2 generation with a homozygous knockout for MCU in Nexexpressing cells (neurons). Results: Genotyping confirmed the successful breeding of such homozygous floxed animals with Cre expression. The transgenic mice are healthy and do not show any conspicuous differences as compared to their non-transgenic littermates. Conclusion: We will use these mice, induce EAE and compare the disease courses of knockout mice with non-transgenic controls. Furthermore, we will analyze $\mathrm{Ca} 2+$-dependent mechanisms of neuronal degeneration by use of histopathological and live imaging techniques.

\section{P6}

The Role of Neurofeedback on Improving the Memory of Elderly People with Alzheimers Disease: a Review Article

\section{Lida Hasanzadeh}

Student Research Committee, Shiraz University of Medical Sciences, Shiraz, Iran

The Neuroscience Journal of Shefaye Khatam, 2019; 7(3): P6

Introduction: human memory naturally decreases after 60 years of age. Alzheimers is one of the most commonly diagnosed dementia diseases in aging. With prevalence over the next few decades. Alzheimers causes cognitive impairments that lead to a decrease in the quality of life and the loss of individual autonomy in everyday life activities. Neurofeedback has a important role for people with intellectual deficits. So that the mind has the ability to regenerate and change pain naturally. In this review study. We intend to study the role of neurofeedback on brain function and memory in the elderly with alzheimers disease. Materials and Methods: The keywords of neurofeedback, memory, elderly, alzheimers were searched in Pubmed, Google scholar and SId in the years of 2008 to 2019.51 articles were the result of our used to write this article. Results: There are various ways to improve the memory status of elderly people with alzheimers disease through various means, including neurofeedback and cognitive exercises that increase frontal lobe and activation of neural circuits for executive functions and memory, and improve the defects of executive functions and memory of elderly it is an alzheimers which affects brain waves and increases the flexibility, which decreases teta waves and increases beta waves, and neurofeedback affects the three sensory, motor, cingulate cortex. There is no positive or negative reinforcement in this method, and it is not necessary to speak during neurofeedback cognitive exercises that increase the wave velocity by reducing slow-wave activity. Conclusion: The neurofeedback is the improve of deformity of EEG that neuripsychological educational methods that can be used to help elderly people with alzheimers disease. 
P7

Sertoli Cells Avert Neuroinflammation-Induced Cell Death and Improve Motor Function and Striatal Atrophy in Rat Model of Huntington Disease

\section{Mahdi Tizro, Abbas Aliaghaei}

Faculty of Medicine, Shahid Beheshti University of Medical Sciences, Tehran, Iran

The Neuroscience Journal of Shefaye Khatam, 2019; 7(3): P7

Introduction: Huntington's diseases (HD) is a genetically heritable disorder, linked with continuing cell loss and degeneration mostly in the striatum. Currently, cell therapy approaches in HD have essentially been focused on replenishing or shielding cells lost over the period of the disease. Materials and Methods: Herein, we sought to explore the in vitro and in vivo efficacy of primary rat sertoli cells (SC) and their paracrine effect against oxidative stress with emphasis on HD. Initially, SC were isolated and immunophenotypically characterized by positive expression ofGATA4. Besides, synthesis of neurotrophic factors of GDNF and VEGF by SC were proved. Next, PC12 cells were exposed to hydrogen peroxide in the presence of conditioned media (CM) collected from $\mathrm{SC}(\mathrm{SC}-\mathrm{CM})$ and cell viability and neurogenesis were determined. Bilateral striatal implantation of SC in 3-nitropropionic acid (3-NP) lesioned rat models was performed and one month later, post-graft analysis was done. Results: According to our in vitro results, CM of SC protected PC12 cells against oxidative stress and remarkably augmented cell viability and neurite outgrowth. Moreover, grafted SC survived, exhibited decreases in both gliosis and inflammatory cytokines levels, and ameliorated motor coordination and muscle activity, together with an increase in striatal volume as well as in dendritic length of the striatum in HD rats. Conclusion: In conclusion, our results indicate that SC provide a supportive environment, with potential therapeutic benefits aimed at HD.

\section{P8}

The Effects of Exercise on Cortical Oxidative Stress Markers and Memory Impairments Induced by Lipopolysaccharide in Rats

\section{Zahra Jahangiri, 2, Zahra Gholamnezhad ${ }^{1,2}$, Mahmoud Hosseini, 3}

${ }^{1}$ Neurogenic Inflammation Research Center, Mashhad University of Medical Sciences, Mashhad, Iran

${ }^{2}$ Department of Physiology, Faculty of Medicine, Mashhad University of Medical Sciences, Mashhad, Iran

${ }^{3}$ Division of Neurocognitive Sciences, Psychiatry and Behavioral Sciences Research Center, Mashhad University of Medical Sciences, Mashhad, Iran
The Neuroscience Journal of Shefaye Khatam, 2019; 7(3): P8

Introduction: Lipopolysaccharide (LPS) is a potent bacterial endotoxin derived from the cell wall of gramnegative bacteria, and neuroinflammation induced by LPS administration is a common animal model of Alzheimer's disease (AD). Physical exercise has an effective role to improve neurodegenerative diseases. In this study, the exercise effects on behavioral tests and cortical oxidative stress of lipopolysaccharide (LPS) administered rats were investigated. Materials and Methods: The rats were divided into four groups $(\mathrm{N}=8)$ : (1) control; (2) moderate training (MT, $15 \mathrm{~m} /$ min, $30 \mathrm{~min} /$ day, 9 weeks); (3) LPS (1 mg/kg LPS) and (4) LPS+MT (1 mg/kg LPS; $15 \mathrm{~m} / \mathrm{min}, 30 \mathrm{~min} /$ day, 9 weeks). LPS was injected two hours before the behavioral experiments during the last week of training. Finally, the rats' brain were removed for biochemical assessments. Results: LPS increased escape latency and traveled distance to reach the platform as well as decreased the time spend and traveled distance in target quadrant of Morris water maze (MWM) test $(P<0.05-P<0.001)$. In the passive avoidance (PA) test, LPS decreased the latency to enter the dark compartment and the time spent in the light compartment and increased the time spent in the dark compartment $(P<0.01-P<0.001)$, while MT improved the rats performances in MWM and PA tests $(P<0.01-P<0.001)$. Additionally, LPS increased malondialdehyde (MDA) and nitric oxide metabolite, and decreased thiol contents and catalase (CAT) and superoxide dismutase (SOD) activity in the cortical tissues compared to the control group $(P<0.01-P<0.001)$; while moderate training decreased the levels of MDA; increased thiol contents and SOD and CAT activity in the LPS+MT compared to the LPS group $(P<0.001)$. Conclusion: These results indicated that moderate training improved LPS-induced learning and memory impairments by attenuating the brain oxidative damage.

P9

The Neuroprotective Effect of Citrus Aurantium Essential Oil on Passive Avoidance Memory in Ischemia Animal Model

Arezo Sadeghi Manesh ${ }^{1}$, Valiallah Khalaji-Pirbalouty ${ }^{2}$, Zahra Lorigooini ${ }^{1}$, Akram Torki ${ }^{1}$, Mahmoud RafieianKopaei ${ }^{1}$

${ }^{1}$ Medical Plants Research Center, Basic Health Sciences Institute, Shahrekord University of Medical Sciences, Shahrekord, Iran

${ }^{2}$ Department of Biology, Faculty of Sciences, Shahrekord University, Shahrekord, Iran

The Neuroscience Journal of Shefaye Khatam, 2019; 7(3): P9

Introduction: Stroke is one of the major causes of disability and the third leading cause of mortality in humans. In recent years, growing attention has been paid to medicinal plants as a potential source of treatment 
for ischemia/reperfusion. The aim of this study was to investigate the effect neuroprotective of Citrus aurantium essential oil on passive avoidance memory in ischemia animal model. Materials and Methods: Fifty adult male rats weighing 250-300 g were randomly divided into five groups: control, sham, ischemia and essential oil (50 and $75 \mathrm{mg} / \mathrm{kg}$ body weight)-treated. Ischemia was induced by clamping the carotid artery for 30 minutes. Then the shuttle box test was performed and the antioxidant capacity of the serum and brain was measured. Results: The passive avoidance memory in the essential oil-treated groups, the difference in time between primary and secondary latency was greater than that in the ischema group. The antioxidant capacity of the brain and serum in the essential oil-treated groups increased compared to the ischema group, and the results in the treatment groups were comparable to the control group. Conclusion: C. aurantium essential oil has neuroprotective effects on passive avoidance memory and antioxidant capacity during ischemia-reperfusion in rat. Probably, this plant essential oil can be used as a complementary therapy for reducing the symptoms of this disorder.

\section{P10}

Diagnosis of MS Plaques Using MRI Protocols(STIR) Before and After Repair of Myelin Sheath by Image Processing

\section{Sogand Abbasi Azizi', Payam Goodarzi², Ali Khanzade Moghadam $^{3}$}

${ }^{1}$ Technologist of Radiology, Iran University of Medical Sciences, Tehran, Iran

${ }^{2}$ Department of Computer Engineering, Islamic Azad University, Tehran, Iran

${ }^{3}$ Shadid Beheshti Hospital, Abadan University of Medical Sciences, Ahvaz, Iran

The Neuroscience Journal of Shefaye Khatam, 2019; 7(3): P10

Introduction: Multiple sclerosis is a chronic inflammatory demyelinating disease of the central nervous system (CNS), which gives rise to focal lesions in the gray and white matter and to diffuse in the entire brain. Inflammation is dominated by major histocompatibility complex Class I restricted T-cells. Activated microglia are seen in close contact with degenerating myelin sheaths, axons, and neurons. The purpose of this study was to design and introduce a diagnosis of MS plaques using MRI protocols (STIR) before and after repair of myelin sheath by image processing. Materials and Methods: This research was a software designing of many MRI images were analyzed with the software designer. The designed software was in MATLAB. In this study; we used image processing techniques such as; noise removing, edge denotation, separate of area with high density and contrast increasing for analysis The results of all lesion diagnostics were analyzed and compared in the pathologist's report. Results: Designed software enables the present MRI images analyzes them pixel by pixel. This software in addition evaluates the areas of lesions and shown them without viewer diagnosis completely. Final results of diagnostic software analysis showed high sensitivity. Conclusion: Contemporary assessments of morphologic and physiologic traits of lesions by a computer aided diagnostic software can improve the radiologist's and neurologist precision and decrease reading time of bulk images of MRI. Using this software to increase the accuracy of the lesions detection is suggested.

\section{P11}

Effect of Pregabalin-Loaded Nanoparticles on Spatial Memory in Pentylenetetrazol- Induced Chemical Kindling Model of Epilepsy

Fereshteh Rezazadeh', Maryam Ghasemi Kasman', Maryam Mohadjerani ${ }^{3}$ Shahram Ghasemi Mir ${ }^{4}$

'Department of Cellular and Molecular Biology, Faculty of Basic Sciences, University of Mazandaran, Babolsar, Iran

${ }^{2}$ Neuroscience Research Center, Health Research Institute, Babol University of Medical Sciences, Babol, Iran

${ }^{3}$ Department of Cellular and Molecular Biology, Faculty of Basic Sciences, University of Mazandaran, Babolsar, Iran

${ }^{4}$ Department of Chemistry, Faculty of Chemistry, University of Mazandaran, Babolsar, Iran

The Neuroscience Journal of Shefaye Khatam, 2019; 7(3): P11

Introduction: Epilepsy is a public health problem and one of the most common and heterogeneous neurological disorders that has a social and economic impact affecting 65 million people worldwide. One of the causes of epilepsy is oxidative stress. Oxidative stress caused cellular disorder, cellular damage and cell death via oxidation of lipids, nucleotides, proteins and finally the oxidation of protein caused changes in body enzymes. Also the oxidative stress caused spatial memory impairment. Pregabalin is used in the treatment of epilepsy, neuropathic pain and general anxiety disorder that has side effects and low half-life. With the help of nanotechnology, this effects can be reduced and more of the drug transmitted to the brain. The aim of this study was to transfer the nano carrier of pregabalin to the brain and survey its effect on memory and spatial learning in the epileptic model. Materials and Methods: In this study, animals were divided into 4 groups. The groups was treated with salin, pregabalin, pregabalin-loaded nanoparticles for 10 days. Epilepsy model was induced by i.p injection of PTZ every second day.Animal behaviors were checked for a period of 20 minute after each PTZ injection. Morris water maze task was used to assess the memory and spatial 
learning of animals. Results: The results of this study show improvement the memory and spatial learning with pregabalin-loaded nanoparticles. Conclusion: The nanoparticles of pregabalin have been able to transfer the drug well into the brain and reduce the damage to the brain.

\section{P12}

Forced Swimming Exercise Alleviates Trigeminal Neuropathic Pain: Role of Oxidative Stress

Zahra Rostami ${ }^{1}$, Sahar Ghasemi ${ }^{1}$, Hamed Farzadmanesh $^{2}$, Manuchehr Safari ${ }^{2}$, Ali Ghanbari ${ }^{3}$

${ }^{1}$ Student Research Committee, Semnan University of Medical Sciences, Semnan, Iran

${ }^{2}$ Department of Anatomical Sciences, Faculty of Medicine, Semnan University of Medical Sciences, Semnan, Iran ${ }^{3}$ Research Center of Physiology, Semnan University of Medical Sciences, Semnan, Iran

The Neuroscience Journal of Shefaye Khatam, 2019; 7(3): P12

Introduction: Neuropathic pain is one of the most debilitating conditions following somatosensory system injuries. Oxidative stress as an important mechanism attracted so much consideration. Along with different pharmacological treatments, exercise as a non-medicinal method has an attenuating role in pain. The aim of this study is, if physical activity reduces trigeminal neuropathic pain, whether or not oxidative stress is involved. Materials and Methods: Twenty-eight female wistar rat, weighting $200 \pm 20 \mathrm{~g}$ were divided into 4 groups (sham, neuropathy, neuropathy + single bout exercise, and neuropathy +2 -week exercise) with 7 rat in each group. Neuropathic pain induced by chronic constriction injury (CCI) of infraorbital nerve using 2 loose ligation around nerve. Mechanical allodynia was evaluated by Von Frey filament. Activity of the serum antioxidant enzymes superoxide dismutase, and glutathione peroxidase were evaluated by related rat ELISA kits. Behavioral data were analyzed by one-way analysis of variance. Enzymes assay data were analyzed through Kruskal-Wallis test. Results: our data showed that a single bout swimming exercise (45 minutes) and 2 weeks (45 minutes/ daily, 6 days per week) significantly reduced neuropathic pain compared to neuropathy $(\mathrm{CCI})$ group $(\mathrm{P}<0.05$ and $\mathrm{P}<0.01)$. Superoxide dismutase level showed no significant difference between groups (fig 2). Glutathione peroxidase level significantly increased $(\mathrm{P}<0.05)$ in group received 2 weeks exercise respect to neuropathy group (fig 3). Conclusion: swimming exercise alleviates trigeminal neuropathic pain through enhancing antioxidant enzyme GPx level probably.

\section{P13}

Comparative Evaluation of Knowledge Level of Shiraz University of Medical Sciences Students about Orofacial Pain and its Managements

\section{Mostafa Rezaei1, Azita Azad ${ }^{1}$ Orkideh Estifaei², Faezeh Marzooghi ${ }^{2}$}

${ }^{1}$ Department of Oral Medicine, Faculty of Dentistry, Shiraz University of Medical Sciences, Shiraz, Iran

${ }^{2}$ Students Research Committee, Faculty of Dentistry, Shiraz University of Medical Sciences, Shiraz, Iran

The Neuroscience Journal of Shefaye Khatam, 2019; 7(3): P13

Introduction: Orofacial pain evaluation and treatment is often a shared responsibility between the dentist and physician. A considerable overlap exists between the two professions. The ability of the health professional is only distinguished by the individual's knowledge and training. This study aims to explore and compare the knowledge of chronic orofacial pain within two final year dental and medical students who studied at Shiraz University of Medical Sciences. Materials and Methods: A questionnaire, containing 15 multiple choice questions on diagnosis and treatment of OFP, was given to 200 randomly selected two final year dental and medical students in both main and international branches of Shiraz University of Medical Sciences. Results: An overall response rate of $94 \%$ was achieved among students. The mean score of knowledge of OFP was found to be $31.52 \pm 11.33$ (of a total of 70). Medical students were significantly more knowledgeable than dental students $(\mathrm{p}=0.00)$. However, no significant difference was recorded between main and international dental students $(p=0.296)$. While, in Medical field in diagnosis section, there was a statistically significant difference between Main and International students $(p=0.024)$. In the treatment section, no significant difference was found between students of main and international branches $(p=0.693)$. Conclusion: According to the results, the level of knowledge of dental students regarding OFP is not desirable. The main medical students may have sufficient knowledge to make an appropriate diagnosis of OFP, but their treatment knowledge level of OFP is low. The result also highlights the knowledge gaps between dental and medical students and perhaps the need for more extensive inclusion of OFP in the dental undergraduate curriculum.

\section{P14}

\section{Implications for the Treatment of Memory Disorders in PTSD by TCSOX229}

\section{Sudabeh Saleh Abadi, Kataneh Abrari, Mahmoud Elahdadi Salmani}

Faculty of Biology, Damghan University, Damghan, Iran

The Neuroscience Journal of Shefaye Khatam, 2019; 7(3): P14

Introduction: The inability to traumatic memory extinction is one of the cognitive impairments in patients with PTSD. Amygdala is one of the structures that interfere with stress effects on different steps of 
memory in these patients. The lateral hypothalamic oroxinergic neurons have extensive bipolar connections with amygdala. Therefore, the purpose of this study was to evaluate the role of orexin receptor 2 in facilitating memory extinction in PTSD. Materials and Methods: In this study, Wistar rats were cannula implanted, bilaterally, in the amygdala for microinjection of orexin receptor-2 antagonist (TCSOX229; TCS-, $30 \mathrm{nmol} /$ side) and the solvent of TCSOX229, respectively in 2 experimental groups; TCS and TCS control groups. One week after the surgery, single prolonged stress (SPS) method used to develop PTSD in rats. A week later, rats were conditioned by receiving electrical foot shock in fear conditioning system. The day after, the freezing behavior, as an indicator of memory extinction, was evaluated in 4 consecutive days. Immediately after every freezing behavior test in first three days, they received microinjection of drugs. Results: The level of freezing did not change during the first test. However, in the two next tests, the amount of immobilization in the TCS group was significantly lower than that of control $(\mathrm{P}<0.001)$. In all tests, the rate of freezing in control group was about 70 to 80 percent, while in TCS group the freezing time decreased from 65 percent to 35 percent. Conclusion: Repeated injection of orexin-2 receptor antagonist into the amygdala of PTSD animals, immediately after the extinction tests, facilitated the process of memory extinction.

\section{P15}

The Role of CSCs/CCs Derived from GBM Patient in Switching of the Healthy Macrophage from M1 to M2

Sara Abdolahi', Maryam Khaleghi Ghadiri', Ali Gorji ${ }^{1,3,4}$

${ }^{1}$ Shefa Neuroscience Research Center, Khatam Alanbia Hospital, Tehran, Iran

${ }^{2}$ Department of Neurosurgery, Westfälische Wilhelms-Universität, Münster, Germany

${ }^{3}$ Epilepsy Research Center and Department of Neurology, Westfälische Wilhelms-Universität Münster, Münster, Germany ${ }^{4}$ Department of Neuroscience, Mashhad University of Medical Sciences, Mashhad, Iran

The Neuroscience Journal of Shefaye Khatam, 2019; 7(3): P15

Introduction: Glioblastoma multiform (GBM) is the most aggressive malignant primary brain tumor. Many factors contribute to the inevitable recurrence of GBM and its resistance to current therapeutics. One of the most commonly detected phenomena in GBM is the abundant macrophage infiltration without apparent phagocytic activity. Accumulating evidences have suggested that the tumor-associated macrophage (TAM) infiltration can be linked to the poor prognosis in GBM. There are controversial reports about the origin, the recruitment, and the functions of the TAMs in GBM. Depending on macrophages/microglia receive which signals from their surroundings, their functional phenotype would be changed. They could be polarized to M1 phenotype in response to an inflammatory condition and produce pro-inflammatory factors such as IL-6 or IL-12. In contrast, in anti-inflammatory condition they would be polarized to M2 phenotype, which characterized by wound healing, angiogenic and pro-tumorigenic properties. So, in this study, we are investigating the role of cancer stem cells/cancer cells derived from GBM patient in switching of the healthy macrophage from M1 to M2. Materials and Methods: Following surgical resection, human brain tumor biopsy was dissociated by mechanical and chemical digestion. Cell pellets were resuspended and cultured to specefic medium. Finally, to characterize the isolated cells, immunostaining was performed. Also we isolated human CD14+ cells from leukoreduction system chamber and then monocytes differentiate into macrophages was done. Flow cytometric analysis of these cells was performed with specific markers. Results: Isolated CSCs/CC from GBM express Nestin, Sox2, Cd133 and Ki67 markers. CD14+ monocytic cells were isolated by CD14 Microbeads. IFN-g was used for macrophage polarization. Purity of monocytes and macrophage were assessed by flow cytometry. Conclution: A better understanding of the interaction of anticancer therapies with innate immunity, and TAMs in particular, may have the way to better patient selection and innovative combination of conventional approaches with immunotherapy.

\section{P16}

The Effect of Piperine on Spatial Memory and Myelin Repair in Local Model of Demylination by Lysolecethin

Hannaneh Roshanbakhsh, 2, Mahmoud Elahdadi Salmani $^{1,2}$, Fereshteh Pourabdolhossein ${ }^{3}$

${ }^{1}$ Student Research Committee, Damghan University, Damghan, Iran

${ }^{2}$ Department of Physiology, Faculty of Biology, Damghan University, Damghan, Iran

${ }^{3}$ Department of Physiology, Faculty of Medical Sciences, Babol University of Medical Sciences, Babol, Iran

The Neuroscience Journal of Shefaye Khatam, 2019; 7(3): P16

Introduction: Multiple sclerosis (MS) is a chronic inflammatory demyelinating disease in the central nervous system. Hippocampal demylination and memory deficit are common in MS patients. Since oxidative stress and inflammatory factors are the most related pathophysiological cause of MS disease, the control of these factors could retard the disease progression and accelerate the myelin repair. Piperine has some 
anti-oxidants, anti-inflammatory and neuroprotective properties which can be a valuable candidate for preventing and improving MS symptoms. Here, we study the effect of piperine on spatial memory, myelin repair process and the gene expression level of inflammatory and anti-inflammatory cytokines, oxidative and antioxidant markers, brain derived neurotrophic factor (BDNF) and myelin basic protein. Materials and Methods: Adult male Wistar rats weighing 200-250 g in 7 experimental groups $(n=8)$ were studied. Demyelination was induced by stereotaxic injection of lysolecithin (LPC) into the CA1 region of the hippocampus. The pre-treatment effect of piperine with a dose of $5 \mathrm{mg} / \mathrm{kg}$ and treatment after demyelination induction in doses of 5, 10 and $20 \mathrm{mg} / \mathrm{kg}$ on spatial memory was assessed by Morris water maze. Myelin repair process was analyzed with myelin specific staining. Real-time PCR technique was used to investigate the effect of piperine on IL$1 \beta$, TNF- $\alpha$, NF-kB, FoxP3, IL-10, iNOS, Hmox-1, Nrf2, BDNF and MBP expression in the hippocampal tissue samples. Also, total antioxidant levels in tissue samples were measured by FRAP biochemical assay. Results: Piperine relatively improved the learning and spatial memory deficit in the LPC- induced demyelination model in the hippocampus. Histological findings also proved that the extent of demyelination was significantly decreased in the treatment and pretreatment groups with piperine. Treatment with piperine also reduced the expression level of the inflammatory cytokines (IL-1 $\beta$, TNF- $\alpha$, NF-kB) and increased the level of anti-inflammatory cytokines (FoxP3, IL-10). Piperine could negatively affect the expression level of iNOS and enhanced the level of antioxidant factors like Hmox-1 and Nrf2. The total antioxidant capacity of tissue was increased in piperine treated groups. Piperine also amplified the expression level of BDNF and MBP. Conclusion: Piperine has neuroprotective effect and can amplify the antioxidant and anti-inflammatory system which facilitates the myelin repair process. Furthermore, piperine improved spatial learning and memory deficit induced by hippocampal demyelination.

\section{P17}

Comparison the Effects of Hesperetin and Hesperetin-NPs on Gene Expression and Microglia Activities Following Demyelination Process in Focal Demyelination Model of Multiple Sclerosis

Saeideh Baradaran', Akbar Hajizadeh Moghaddam ${ }^{1}$, Maryam Ghasemi-Kasman ${ }^{2,3}$

${ }^{1}$ Department of Biology, Faculty of Basic Sciences, University of Mazandaran, Babolsar, Iran

${ }^{2}$ Cellular and Molecular Biology Research Center, Health Research Institute, Babol University of Medical Sciences, Babol, Iran

${ }^{3}$ Neuroscience Research Center, Health Research Institute, Babol University of Medical Sciences, Babol, Iran
The Neuroscience Journal of Shefaye Khatam, 2019; 7(3): P17

Introduction: Demyelination and disturbance of action potential conductance are regarded as main signs of Multiple Sclerosis(MS). More than $70 \%$ percent of patients show visual disorder as the earliest symptoms of MS. Although hesperetin (Hst) has been introduced as effective anti-inflammatory agent in treatment of several inflammatory disorders, but because of low water solubility, its clinical application has been limited. The present study design to evaluate the effects of hesperetin and hesperetin NPs on gene expression, myelin repair and microglia activation in lysolecithin (LPC)-induced demyelination. Materials and Methods: Demyelination process started with injection of LPC $(1 \%, 2 \mu \mathrm{L})$ into rat optic chiasm. Animals have received oral administration of Hst and Hst NPs at dose of $20 \mathrm{mg} / \mathrm{kg}$ for 14 or 21 days' post lesion. Visual evoked potential (VEPs) records were used to assess the functional property of the optic pathway on days $0,7,14$ and 21 post lesions. Immunostaining against Iba 1 (Microglia marker) was carried out for evaluation of inflammation level and glial activation. we measured expressions of MBP (Myelin Basic Protein) mRNAs for determination of remyelination processes. Results: Electrophysiological evidence demonstrated that oral administration of $\mathrm{Hst}$ and Hst NPs could reduce the P1-N1 waves compared to the LPC groups .Immunostaining showed oral administration of Hst NPs effectively ameliorated microglia activation and level of inflammation in optic chiasm area. Results of Real Time PCR indicated that .remyelination process enhanced in the optic chiasm following administration of Hst NPs. Conclusion: Overall, our findings indicate that hesperetin NPs could remarkably enhance the functional recovery of the optic pathway by its protective effects on myelin sheath, attenuation of glial activation and increase of MBP expression.

\section{P18}

Prevalence of Genetic Polymorphisms ABCB1 / C3435T in Patients with Epilepsy in the Population of Fars Province

\footnotetext{
Behnoush Miladpour ${ }^{1}$, Mojdeh Owji ${ }^{2}$, Adib Valibeygi ${ }^{2}$

${ }^{1}$ Department of Biochemistry, Faculty of Medicine, Fasa University of Medical Sciences, Fasa, Iran

${ }^{2}$ Student Research Committee, Faculty of Medicine, Fasa University of Medical Sciences, Fasa, Iran
}

The Neuroscience Journal of Shefaye Khatam, 2019; 7(3): P18

Introduction: $\mathrm{ABCB} 1 / \mathrm{C} 3435 \mathrm{~T}$ gene polymorphism encodes a drug transporter named P-glycoprotein Inhibition which has role in sending xenobiotic outside the cells and therefore affects the plasma level of drugs. In this study the frequency of ABCB1/C3435T 
polymorphism was compared between the epileptic patients and control group. Also, resistance to antiepileptic drugs (AED) was evaluated in different polymorphisms of this gene. Material and Methods: At first, DNA was extracted from blood samples of patients and healthy individuals. Polymorphism of ABCB1/ C3435T gene was analyzed in a group of patients $(n=$ $50)$ and healthy population $(n=100)$ by PCR and RFLP techniques .The frequency of different polymorphism of TT, TC, CC and T, C alleles compared between the patients and control group using SPSS, chi square test and independent $\mathrm{T}$ test. Also, association between different polymorphisms of this gene (TT, TC and CC) and response to AEDs was evaluated by Pearson chisquare test. Results: Of total 50 patients in this study, $20 \%$ had TT, 64\% had TC and 16\% had CC genotype and in the control group $(n=100)$ the result were $28 \%$ TT, 52\% TC and 20\% has CC genotype. We found no statistically significant difference in the genotypic distribution between patients and controls ( $p$-value: 0.371). Also, there was no significant difference between incidence of epilepsy and $\mathrm{C}, \mathrm{T}$ alleles in both patient and control groups ( $\mathrm{p}$-value 0.743 ). An association was observed between TC genotype and response to AED (p-value 0.036). Conclusion: Following the obtained results, there is no significant difference in frequency of ABCB1-C3435T polymorphisms between patients and control group, but $42 \%$ of patients with TC genotype had response to AED significantly, which can show that $\mathrm{C}$ and $\mathrm{T}$ alleles may boost each other's effect in order to improve responsiveness to AED in epileptic patients.

\section{P19}

\section{Methamphetamine Provokes Neuronal Atrophy in Prefrontal Cortex}

\section{Ava Modirzadeh Tehrani, Mahdi Eskandarian Boroujeni $^{2}$, Abbas Aliaghaei, Mohammad Ali Hosseinpour Feizi', Reza Safaralizadeh ${ }^{1}$}

${ }^{1}$ Department of Animal Biology, Faculty of Natural Sciences, University of Tabriz, Tabriz, Iran

${ }^{2}$ Department of Stem Cells and Regenerative Medicine, Faculty of Medical Biotechnology, National Institute of Genetic Engineering and Biotechnology, Tehran, Iran

${ }^{3}$ Department of Cell Biology and Anatomical Sciences, Faculty of Medicine, Shahid Beheshti University of Medical Sciences, Tehran, Iran

The Neuroscience Journal of Shefaye Khatam, 2019; 7(3): P19

Introduction: Methamphetamine (METH) is an addictive psychostimulant drug that induces damage on brain reward system. Prefrontal cortex (PFC) is a part of mesocordical pathway which plays an essential role in drug addiction. Recent reports highlighted that METH induces neurotoxicity via several pathways namely neuroinflammation and apoptosis. The present study is performed to examine the alteration of inflammation and apoptosis pathways in PFC under the influence of METH. Materials and Methods: the male rats were intraperitoneal injected with $15 \mathrm{mg}$ METH (5mg in first day and $10 \mathrm{mg}$ in second day). The rats were euthanized and decapitated $24 \mathrm{~h}$ after last injection. RNA was extracted from PFC tissue. Then the relative expression level of TNF $\alpha$, IL1 $\beta$ and IL10 (as inflammation associated genes), CASP8 (extrinsic apoptosis related candidate) as well as AKT and mROR (regulatory related candidates) were evaluated in comparison with controls. Results: The qPCR results displayed a significant increase in expression of pro-inflammatory cytokine $\mathrm{TNF} \alpha$ and IL1 $\beta$ along with remarkably decreases in anti- inflammatory cytokine IL10 in METH treated groups. Moreover, METH augmented the expression level CASP8 considerably. The relative expression of AKT and mTOR were significantly declined upon METH treatment. Conclusion: METH induced neuronal atrophy in PFC via activation of the neuro inflammation and apoptosis pathways.

\section{P20}

\section{Terminalia Chebula Protects Microglial Cells Against} LPS-Induced M1/M2 Imbalance

\section{Vafa Baradaran Rahimi', Vahid Reza Askari ${ }^{2,3}$}

${ }^{1}$ Student Research Committee, Department of Pharmacology, Faculty of Medicine, Mashhad University of Medical Sciences, Mashhad, Iran

${ }^{2}$ Neurogenic Inflammation Research Center, Mashhad University of Medical Sciences, Mashhad, Iran

${ }^{3}$ Pharmacological Research Center of Medicinal Plants, Mashhad University of Medical Sciences, Mashhad, Iran

The Neuroscience Journal of Shefaye Khatam, 2019; 7(3): P20

Introduction: Terminalia chebula (TC) has been traditionally used in the Iranian traditional medicine (ITM) and Ayurvedic medicine primarily for neurologic disorders and inflammation. Mainly, its fruits have been applied for CNS disorders. The effects of Terminalia chebula as herbal medicine with anti-inflammatory and anti-oxidant properties were aimed on lipopolysaccharide (LPS)-induced microglial inflammation. Material and Methods: Cytotoxicity of TC extract $(0-80) \mu \mathrm{g} / \mathrm{ml}$ on microglial cells was evaluated using the MTT assay. Also, the protective effect of TC extract concentrations with specified amount of LPS-induced mice microglial cells was studied. The concentrations of TNF- $\alpha$ (Tumor Necrosis Factor- $\alpha$ ), IL-1 $\beta$ (Interleukin-1 $\beta$ ), IL-6 and PGE-2 (Prostaglandin-E2) were evaluated using ELISA. Gene expression of TNF- $\alpha$, IL- $1 \beta$, IL6, COX-2 (Cyclooxygenase-2), iNOS and arginase-1 was also evaluated using the Real-Time PCR method. Nitrite oxide and urea were measured using biochemical methods. Results: The studied concentrations of TC 
extract did not affect the viability of microglial cells but significantly protected the viability after treatment with LPS. The concentrations and expression levels of pro-inflammatory factors (TNF- $\alpha$, IL-1 $\beta$, IL-6, PGE2 , and COX-2) were significantly decreased after TC extract treatment in LPS-induced microglial cells with dose dependent manner. The extract also significantly decreased the levels of nitric oxide, increased urea and down regulated the expression of nitric oxide synthesis while arginase-1 expression was enhanced. Conclusion: Our results suggest that TC extract reduces inflammation in microglial cells and can be used as a potential anti-inflammatory agent in central nervous system inflammatory diseases.

\section{P21}

Effects of Phenobarbital on Rat Model of Brain Ischemia

\section{Maryam Salari', Hassan Abbassian ${ }^{2}$}

${ }^{1}$ Department of Physiology, Mashhad University of Medical Sciences, Mashhad, Iran

${ }^{2}$ Department of Neuroscience, Mashhad University of Medical Sciences, Mashhad, Iran

The Neuroscience Journal of Shefaye Khatam, 2019; 7(3): P21

Introduction: There are some molecular pathways in tissue damage in Ischemic Brain Insult. Attentions focused on applying for antioxidants and free radical scavengers. Oxidative stress is caused by excessive production of reactive oxygen species such as hydroxyl radical, superoxide anion radical and hydrogen peroxide. GABAergic agents are used previously for protective effects in ischemia state. In this study we aimed to test potential protective effects of phenobarbital in tissue level in rat's brain. Materials and Methods: Male Wistar Rats 250-350 g were kept in constant condition (12 hr. day, night and temperature) with Food and Water ad libitum. The animals were divided into 4 groups of 8 including: Group 1, was negative control in which ischemia was done and $1 \mathrm{mg}$ normal saline injected IP. Group 2, positive control in which ischemia induction and $100 \mathrm{mg} / \mathrm{kg}$ phenytoin injected IP as a standard neuroprotective agent. Group 3, phenobarbital $20 \mathrm{mg} /$ $\mathrm{kg}$ was injected intraperitoneal. Brain global ischemia was done using Four Vessel occluding (4VO) method expatiated by Pulsinelli et al. with some modification. Briefly, vertebral arteries cauterized permanently via Alar foramina in first vertebra of rat under general anesthesia, after 24 hours carotid arteries occluded for duration of 20 minute before reperfusion and recovery state. 72 hours later brain removed and immediately fixed with formaldehyde $10 \%$. After tissue processing, thin slice (2-4 Microns) prepared and stained for hematoxyline and eosin. Results: Ischemic neuronal cells was seen in control group around the brain tissues specially in hippocampus, but some degree of necrosis seen in other area of brain such as cerebellum, basal ganglia and cortex. Despite slightly ischemic cellular changes only in some area, Phenobarbital group were not have vast necrosis cell around tissues.

\section{P22}

Prevention of Glutamate-Induced Neurotoxicity in Aging Brain by Certain Types of B Vitamins

\section{Manoochehr Messripour ${ }^{1}$, Ali Abedi Madiseh ${ }^{2}$ \\ ${ }^{1}$ Department of Clinical Biochemistry, Faculty of Pharmaceutical Sciences, Isfahan Medical Sciences University, Isfahan, Iran \\ ${ }^{2}$ Department of Basic Sciences, Najafabad Branch, Islamic Azad University, Najafabad, Iran}

The Neuroscience Journal of Shefaye Khatam, 2019; 7(3): P22

Introduction: Glutamate (Glu) acts as an excitatory neurotransmitter in the brain. Excessive levels of Glu in the brain are cytotoxic and lead to several neurodegenerative processes. Rapid removal of the released Glu in synaptic cliff may prevent the excessive excitation of Glu receptors. The enzymes glutamate decarboxylase (GAD), glutamate transaminases (GOT and GPT) glutamine synthetase (GS) use Glu as substrate and have curtail role in maintaining Glu concentrations below excitatory levels in the brain synaptic cleft. Since Glu-induced neurotoxicity play a key role in Alzheimer's disease and Parkinson disease's, this study was undertaken to investigate the efficacy of vitamins B6, vitamin B12 and folic acid on the activities of GAD, GOT, GPT and GS in aging rat brain. Materials and Methods: Male Wistar rats (3 and 30 months old) were used. Each group were assigned to 3 treatments subgroups: (group I) B6 vitamin rats; (group II) B12 vitamin rats; and (group III) foliate rats. The animals were injected with the vitamins $(10 \mathrm{mg} / \mathrm{Kg} /$ day $)$ for 30 days and the day after the last injection the animals were killed by decapitated. Forebrains were removed and homogenized in phosphate buffer $(\mathrm{pH}=7.4)$. This homogenate was centrifuged and the enzyme activities were measured in the clear supernatant. The enzymes were measured in the supernatant. Results: The enzyme activities in aged rat brain were considerably lower compared to young animals. Vitamin B6 induced activation of GAD, GOT and GPT in both ages, but, the differences were more pronounced in aged animals. Vitamin B12 and folic acid stimulate the activity of GS in both young and old animals, but had limited effects on GAD, GOT and GPT of both ages. Conclusion: It is concluded that Glu metabolism might be considered as a therapeutic target for prevention of neurodegenerative disorders and age related symptoms.

P23

Cinnamaldehyde Improves Methamphetamine- 
Induced Spatial Learning and Memory Deficits and Restores ERK Signaling in the Rat Prefrontal Cortex Leila Etemad ${ }^{1}$, Mohammad Saeed $^{2}$, Ameneh Ghadiri'
Ali Roohbakhsh

${ }^{1}$ Pharmaceutical Research Center, Pharmaceutical Technology Institute, Mashhad University of Medical Sciences, Mashhad, Iran

${ }^{2}$ Faculty of Pharmacy, Mashhad University of Medical Sciences, Mashhad, Iran

${ }^{3}$ Department of Internal Medicine and Medical Specialties, Sapienza University of Rome, Rome, Italy

${ }^{4}$ Medical Toxicology Research Center, Faculty of Medicine,

Mashhad University of Medical Sciences, Mashhad, Iran

The Neuroscience Journal of Shefaye Khatam, 2019; 7(3): P23

Introduction: Methamphetamine is a stimulant compound that penetrates readily into the central nervous system. Repeated exposure to methamphetamine leads to damage in the dopaminergic and serotonergic axons of selected brain regions. Previous studies showed that cinnamaldehyde improved memory impairment in animals. In the present study, we aimed to elucidate the effects of cinnamaldehyde on methamphetamineinduced memory impairment in rats. Materials and Methods: Male Wistar rats received methamphetamine (10 $\mathrm{mg} / \mathrm{kg}$, intraperitoneally) for 7 days. Thirty minutes before each injection, animals were given cinnamaldehyde $(20,40$, or $80 \mathrm{mg} / \mathrm{kg})$ or rivastigmine (1 $\mathrm{mg} / \mathrm{kg}$ ). The spatial learning and memory were examined using the Morris water maze test. The expression of extracellular signal-regulated kinase (ERK) phosphorylation in the frontal cortex and hippocampus was also detected by immunohistochemical method. Results: Administration of methamphetamine increased the latency to find the platform in the learning phase, while administration of cinnamaldehyde $(40 \mathrm{mg} / \mathrm{kg})$ or rivastigmine before methamphetamine reversed the increased latency. Administration of cinnamaldehyde, at the dose of $40 \mathrm{mg} / \mathrm{kg}$ with methamphetamine, increased the time and distance traveled in the target quadrant in comparison with the amphetamine group. Moreover, the methamphetamine and cinnamaldehydetreated group had higher expression of phosphorylated ERK1/2 in the prefrontal cortex in comparison with the methamphetamine-treated animals. Conclusion: The present data demonstrated that repeated METH administration impaired cognitive performance through the ERK pathway and decreased the phosphorylation of ERK1/2 in the prefrontal cortex while administration of cinnamaldehyde restored both effects. Accordingly, cinnamaldehyde may be a valuable therapeutic tool for the treatment of cognitive deficits associated with methamphetamine consumption.
Suppression of Neuroinflammation by Platelet-Rich Plasma Improves Functional Recovery in the Rat Model of Spinal Cord Injury

Morteza Gholaminejhad, Gholamreza Hassanzadeh,
Ibrahim Mohammed, Mohammad Akbari, Sahar Ijaz

Department of Anatomy, Faculty of Medicine, Tehran University of Medical Sciences, Tehran, Iran

The Neuroscience Journal of Shefaye Khatam, 2019; 7(3): P24

Introduction: Spinal cord injury (SCI) is serious damage with many clinical complications on patients. The pathophysiology of SCI involves a primary mechanical injury that is followed by a secondary phase. The inflammation is one of the most important processes in secondary injury. If inflammation is not controlled, it leads to expansion of the damage. Platelet-rich plasma (PRP) is an autologous blood-derived product. PRP is a key source of growth factors and anti-inflammatory ingredients involved in tissue repair and regeneration. Materials and Methods: Adult male Wistar rats $(n=21)$ were assigned to 3 groups: control, SCI and SCI+PRP groups. The SCI was created at the T10 level. $10 \mu \mathrm{l}$ PRP was injected intrathecally 24 hours after SCI. After 3 days the spinal cord samples were collected and the level of TNF- $\alpha$, IL-1 $\beta$, and IL- 6 was measured. The Basso, Beattie, Bresnahan (BBB) locomotor rating scale was used at 1, 3, 7, 14, 21 and 28 days after SCI. Also, hematoxylin and eosin (H\&E) staining was performed at day 28 for spinal cord tissue analysis. Results: The level of TNF- $\alpha$, IL-1 $\beta$, and IL- 6 significantly increased after SCI. The level of these pro-inflammatory cytokines significantly decreased in the SCI+PRP group compared with the SCI group. In addition, the comparison between SCI and treatment groups determined a significant reduction in tissue degeneration and the volume of cavities in the SCI+PRP group. Also, BBB scores were significantly increased in PRP-treated rats on days 14, 21 and 28 after injury compared with the SCI group. Conclusion: By reducing the inflammation after SCI, PRP could prevent spinal cord degeneration and the death of neural cells and improve motor function in the rat. By conducting further studies, PRP can be a good option to regulate neuroinflammation.

\section{P25}

Neuronal Differentiation Affects Lingo-1 Variants Expression, Implications in Demyelinating Diseases

Khadijeh Hashemi ${ }^{1}$,Hesam Dehghani ${ }^{2,3}$, Mohammad Mehdi Ghahramani Seno ${ }^{3}$, Mohammadreza Bassami ${ }^{1}$, Amir Afkhami Goli ${ }^{3}$

${ }^{1}$ Department of Biotechnology, Faculty of Veterinary Medicine, Ferdowsi University of Mashhad, Mashhad, Iran

${ }^{2}$ Department of Stem Cell Biology and Regenerative Medicine Research, Institute of Biotechnology, Ferdowsi University of 
Mashhad, Mashhad, Iran

${ }^{3}$ Department of Basic Sciences, Faculty of Veterinary Medicine, Ferdowsi University of Mashhad, Mashhad, Iran

The Neuroscience Journal of Shefaye Khatam, 2019; 7(3): P25

Introduction: Lingo-1 is a Leucine-rich repeat and Ig domain-containing protein which is known as a powerful negative regulator of myelination and oligodendrocyte differentiation. Following the detection of different Lingo-1 variants and the main role of Lingo-1 expression in various CNS diseases such as Multiple sclerosis, Alzheimer, Neuroblastoma and Glioblastoma we focused on neuronal differentiation and its effects on the mRNA expression of lingo-1 variants. Materials and Methods: Neuro2A cells with the convenient characteristic and ability to differentiate were used in this study. Differentiation was triggered by diverse concentration of Caffeine, Retinoic acid and BSA, as well as serum starvation. The neurite outgrowth was measured during various time points. Furthermore, the transcript levels of Lingo-1 and HPRT were evaluated by RT-PCR. Results: Results showed that Neuro2A cells as an undifferentiated neuronal cell line with neuronal and amoeboid stem cell morphology express only variant 1 of lingo-1. Fully differentiated neurons showed high levels of variant 2 , as well as variant $1 \mathrm{mRNA}$. Also the FBS $0.01 \%$, Retinoic acid 10uM and BSA 0.1\% (24h and $48 \mathrm{~h}$ ) was shown to be the best condition for neuronal differentiation, although variant- 2 would be expressed regardless of differentiation protocol. Conclusion: These findings may offer new pharmacological opportunities in order to target the most convenient variant of lingo-1, preserving the other lingo- 1 functions in the body to lessen the unwanted side effects.

\section{P26}

Comparing Effects of Nicorandil and Dimethyl Fumarate on Stereological Parameters of the Brain in the Rat Model of Brain Ischemia

Maryam Owjfard ${ }^{1,4}$, Mohammad Reza Bigdeli, 2, Anahid Safari ${ }^{3}$, Mohammad Reza Namavar, 5

${ }^{1}$ Department of Animal Sciences and Biotechnology, Faculty of Life Sciences and Biotechnology, Shahid Beheshti University, Tehran, Iran

${ }^{2}$ Institute for Cognitive and Brain Sciences, Shahid Beheshti University, Tehran, Iran

${ }^{3}$ Stem Cells Technology Research Center, Shiraz University of Medical Sciences, Shiraz, Iran

${ }^{4}$ Clinic Neurology Research Center, Shiraz University of Medical Sciences, Shiraz, Iran

${ }^{5}$ Histomorphometry \& Stereology Research Center, Shiraz University of Medical Sciences, Shiraz, Iran

The Neuroscience Journal of Shefaye Khatam, 2019; 7(3): P26

Introduction: Cell death is the critical event for the pathogenic mechanism of cerebral ischemia/ reperfusion (I/R) injury. Dimethyl fumarate (DMF) has anti-inflammatory and immune-modulatory effects on cerebral ischemia and Nicorandil protects the brain against ischemic injury via its anti-inflammation and anti-apoptotic characteristics. The aim of this study was to investigate the therapeutic effects of these drugs on behavioral and histological changes in rats' brain after transient middle cerebral artery occlusion (MCAO) . Materials and Methods: 24 rats were randomized into four groups, including the sham, vehicle-treated I/R, DMF-treated I/R and Nicorandil-treated I/R groups with oral gavage for 3 days. Cerebral I/R injury were induced by a transient MCAO for $1 \mathrm{~h}$, followed by $72 \mathrm{~h}$ of reperfusion. Neurobehavioral scores were evaluated for three days. Histological processing, the total and infarct volume and also numerical density and total number of neurons, non-neurons and dead neurons in right cortex were estimated by unbiased stereological methods. Results: Although brain ischemia treatment with DMF did not have significant effect on the infarction size, it significantly improved neurobehavioral function, increased numerical density of neurons and decreased numerical density of dead neurons upon acute ischemic. However, Nicorandil treatment showed a significant reduction in the infarction volume especially in the striatum without significant effect on neurobehavioral function and numerical density of neurons, non-neurons and dead neurons. Conclusion: Although Nicorandil did not have significant effects on brain ischemia, DMF treatment supports neuronal survival and neurobehavioral improvement in ischemic stroke, while its potency is limited in the infarction size.

\section{P27}

Effect of Levodopa and Benserazide and Combinational Therapy on Histopathological Changes in SNC and Hippocampus and Also Oxidative Stress Enzymes in Serum of the Parkinsonian Rats

Hamed Farzadmanesh ${ }^{1}$, Mahmoud Reza Madadian', Marzyeh Salami', Ali Ghanbari', Majid Mir Mohammad Khani ${ }^{5}$, Manouchehr Safari ${ }^{1}$

${ }^{1}$ Nervous System Stem Cells Research Center, Department of Anatomy, Semnan University of Medical Sciences, Semnan, Iran ${ }^{2}$ Faculty of Medicine, Branch of North Tehran, Islamic Azad University, Tehran, Iran

${ }^{3}$ Department of Biochemistry, Semnan University of Medical Sciences, Semnan, Iran

${ }^{4}$ Department of Physiology, Semnan University of Medical Sciences, Semnan, Iran

${ }^{5}$ Department of Epidemiology, Semnan University of Medical Sciences, Semnan, Iran

The Neuroscience Journal of Shefaye Khatam, 2019; 7(3): P27 
Introduction: Parkinson's disease is a progressive and chronic disorder of the central nervous system. Levodopa and dopaminergic agonists are not used alone, but also combined with other drugs such as benserazide. The aim of this study was to investigate the effects of levodopa and benserazide on histopathological changes in the substantia nigra, and the changes of superoxide dismutase and glutathione peroxidase enzymes in serum of the Parkinsonian rats. Materials and Methods: Twenty male Wistar rats were studied in this project. Mouses were divided into five groups: control, sham, levodopa, benserazide, levodopa and benserazide. Levodopa was administered at a dose of $10 \mathrm{mg} / \mathrm{kg}$, benserazide at a dose of $20 \mathrm{mg} / \mathrm{kg}$, and in the combined group, $30 \mathrm{mg} / \mathrm{kg}$ intraperitoneally (ip) were injected into the mouses. Results: The overall comparison of the five groups showed a significant difference between the groups for the level of SOD enzyme $(p=0.002)$. The post hoc test showed a significant difference between the combination group of levodopa and benserazide with levodopa $(p=0.035)$ and benserazide $(p=0.001)$ groups, so that the SOD level in the combination group was $10.73 \pm 3.1$ and $15.93 \pm 3.1$ respectively, which was more than the previous two groups, but the level of the GPX enzyme did not show significant differences between the groups $(p=0.084)$. About the histopathological changes, counting the number of cells showed a significant difference between the combinational group (levodopa+benserazide) compared to the MPTP group. Conclusion: According to the results, increasing SOD levels will neutralize oxidative stress factors and free radicals, which will improve the cell's efficiency and survive.

\section{P28}

Protective Effect of Vitamin C Against CisplatinInduced Memory and Learning Impairment in Male Rats

Marzieh Kafami', Masoud Hoseinzadeh ${ }^{2}$, Amir Alizadeh $^{3}$, Parniaan Heydari ${ }^{3}$, Samira sabeti ${ }^{2}$

${ }^{1}$ Cellular and Molecular Research Center, Department of Physiology and Pharmacology, Faculty of Medicine, Sabzevar University of Medical Sciences, Sabzevar, Iran

${ }^{2}$ Department of Physiology and Pharmacology, Faculty of Medicine, Sabzevar University of Medical Sciences, Sabzevar, Iran

${ }^{3}$ Student Research Committee, Sabzevar University of Medical Sciences, Sabzevar, Iran

The Neuroscience Journal of Shefaye Khatam, 2019; 7(3): P28

Introduction: Cisplatin is widely used for cancer therapy. Although cisplatin is very effective drug, it is highly toxic and has lots of side effects. The present study is conducted to assess influence of the vitamin $\mathrm{C}$ (Vit $\mathrm{C}$ ) on learning and memory impairments in cisplatin-induced cognitive impairment. Material and Methods: The thirty two male rats divided into four groups: Group I (Control; $\mathrm{n}=8$ ) received 0.9\% saline once per day for a week intraperitoneally (i.p); Group II (cisplatin; $\mathrm{n}=8$ ) received $2 \mathrm{mg} / \mathrm{kg} / 7$ day cisplatin $\mathrm{ip}$; Group III (cisplatin+Vit C; $\mathrm{n}=8$ ) received $2 \mathrm{mg} / \mathrm{kg} / 7$ day, i.p. cisplatin and vitamin C $150 \mathrm{mg} / \mathrm{kg} / 7$ day, i.p; Group IV received $150 \mathrm{mg} / \mathrm{kg} / 7$ day vitamin $\mathrm{C}$. At the end of the experiment, the memory and learning were evaluated by Morris water maze (MWM) and passive avoidance (PA) tests. Results: Cisplatin increased the escape latency and traveled path in MWM $(\mathrm{P}<0.001)$. Cisplatin also shortened the latency for enter the dark room of PA as well as the time spent in the target quadrant in probe trial test of MWM $(\mathrm{P}<0.05-\mathrm{P}<0.001)$. All the effects of cisplatin were reversed by Vit $\mathrm{C}(\mathrm{P}<0.05-\mathrm{P}<0.01)$. Conclusion: The results of the present study showed that Vit $\mathrm{C}$ improves cisplatin-induced memory and learning impairment.

\section{P29}

Effect of Vitamin E on Cisplatin-Induced Memory Impairment in Male Rats

Masoud Hoseinzadeh ${ }^{1}$, Amir Alizadeh ${ }^{2}$, Parniaan Heydari' $^{2}$ Mahmoud Hosseini ${ }^{3}$, Marzieh Kafami', Farimah Beheshti ${ }^{5}$, Narges Marefati ${ }^{5}$, Zohreh Arab ${ }^{5}$, Mustafa Ghanbarabadi ${ }^{4}$

${ }^{1}$ Department of Physiology and Pharmacology, Faculty of Medicine, Sabzevar University of Medical Sciences, Sabzevar, Iran

${ }^{2}$ Student Research Committee, Sabzevar University of Medical Sciences, Sabzevar, Iran

${ }^{3}$ Division of Neurocognitive Sciences, Psychiatry and Behavioral Sciences Research Center, Mashhad University of Medical Sciences, Mashhad, Iran

${ }^{4}$ Cellular and Molecular Research Center, Department of Physiology and Pharmacology, Faculty of Medicine, Sabzevar University of Medical Sciences, Sabzevar, Iran ${ }^{5}$ Department of Physiology, Faculty of Medicine, Mashhad University of Medical Sciences, Mashhad, Iran

The Neuroscience Journal of Shefaye Khatam, 2019; 7(3): P29

Introduction: Neurotoxicity is one of the adverse effects of cisplatin (CP) due to inflammation and oxidative stress in central nervous system. In present study we assess the protective effects of vitamin E (Vit E) on learning and memory impairments in cisplatin-induced cognitive impairment and hippocampus tissues oxidative damage. Materials and Methods: Methods four animal groups ( $n=7$ for each group) were used as follows: (1) control, (2) cisplatin (2 mg/kg/7day, i.p), (3) cisplatin+ Vit E (200 mg/kg/7day, i.p), (4) Vit E (200 mg/kg/7day, i.p). The spatial memory was evaluated by using Morris water maze test (MWM) after one week. Hippocampus concentration of Malondialdehyde (MDA), thiol 
and superoxide dismutase (SOD) were measured by biochemical methods. Results: Cisplatin significantly increased the escape latency but decreased the percentage of time spent and traveled pathway in target quadrant on final trial test day $(\mathrm{p}<0.05)$. Supplementation with Vit E significantly revers all results of spatial memory test $(p<0.001)$. Biochemical data indicated that Vit $\mathrm{E}$ decreased MDA but increased thiol and SOD activity $(p<0.05)$. Conclusion: Our findings have shown that Vit E improves cisplatin-induced memory impairment possibly through effect on hippocampal oxidative status.

\section{P30}

Effects of Fennel Hydroalcoholic Extract on Passive Avoidance Memory in Harmaline Induced Tremor Rats

Niloufar Jafari', 2, Leila Amini Noghondar', ', Hassan Abbassian ${ }^{2}$

${ }^{1}$ Student Research Committee, Faculty of Medicine, Mashhad University of Medical Sciences, Mashhad, Iran

${ }^{2}$ Department of Neurosciences, Faculty of Medicine, Mashhad University of Medical Sciences, Mashhad, Iran

The Neuroscience Journal of Shefaye Khatam, 2019; 7(3): P30

Introduction: Essential tremor (ET) is a neurodegenerative dementia with partial to total cognitive and physical impairments which includes over $5 \%$ of people worldwide. We still don't have definite treatment for ET. This study sets out to assess the effect of the Fennel alcoholic extract on passive avoidance memory of tremor induced rat models. Materials and Methods: 40 male rats (weighing 40-60 g) were kept in individual cages with access to food and water ad-libitum in a $12 / 12$ dark/light cycle. The animals divided in 5 groups of 8 , including control saline, Harmaline $10 \mathrm{mg} / \mathrm{kg}$, Fennel 50, 100, and $200 \mathrm{mg} / \mathrm{kg}$ IP. Animals placed in the light chamber of the shuttle - box, the door opened and allowed to enter the dark sector without electric shocks. When the animal entered the dark sector, an electric pulse was administered to cage floor $(0.5 \mathrm{~A}, 5 \mathrm{~ms})$. In retention trials, $24 \mathrm{~h}$ after training, the test performed to evaluate memory; so the animal was placed in the light arena of the shuttle-box apparatus. After $30 \mathrm{~s}$, the door opened and the time required to enter the dark sector was recorded as retention time (step-through latency (STL)). Time in the dark compartment (TDC) was recorded as an indicator of contextual memory. Results: STL in Harmaline group was significantly decreased. Significant STL improvement in fennel groups was observed in comparison with Harmaline group, but different drug dosage didn't show any correlation $(51.7 \pm 6.07,51 \pm 7.08$, $52.7 \pm 6.49 v s .34 .4 \pm 4.62)$. Although no TDC significant difference was detected between different fennel groups, there was a meaningful TDC decrease in all experimental group in comparison with Harmalin group (84.2 \pm 14.50 ,
93.4 $\pm 12.39,94.8 \pm 11.27 v s .129 .4 \pm 39.43)$. Conclusion: present study revealed that fennel hydroalcoholic extract can be considered in tremor treatment protocols.

\section{P31}

Pioglitazone Prevented from Learning and Memory Impairment and Nitric Oxide Increment Induced by Scopolamine

\section{Maryam Salari ${ }^{1}$, Farimah Beheshti, ${ }^{2,3}$, Seyedeh Elnaz nazari $^{1}$, Sadegh Shabab ${ }^{1}$, Mahmoud Hosseini ${ }^{1}$}

${ }^{1}$ Division of Neurocognitive Sciences, Psychiatry and Behavioral Sciences Research Center, Mashhad University of Medical Sciences, Mashhad, Iran

${ }^{2}$ Neuroscience Research Center, Torbat Heydariyeh University of Medical Sciences, Torbat Heydariyeh, Iran

${ }^{3}$ Department of Physiology, Faculty of Paramedical Sciences, Torbat Heydariyeh University of Medical Sciences, Torbat Heydariyeh, Iran

The Neuroscience Journal of Shefaye Khatam, 2019; 7(3): P31

Introduction: The aim of present study was to evaluate the effects of pioglitazone (Pio) on learning and memory impairment and nitric oxide (NO) increment induced by Scopolamine (Sco). Materials and Methods: Male Wistar rats were injected by saline, Sco or three doses including 10, 20 and $30 \mathrm{mg} / \mathrm{kg}$ of Pio before Sco. Passive avoidance (PA) test and morris water maze (MWM) were done and then the hippocampus was removed for biochemical measurements. Results: Sco decreased the latency to enter the dark in PA test. It also increased the total time spent dark. In a MWM, the Sco group significantly took a longer period of time and traveled a greater distance to reach the platform, which was prevented by Pio. Malondialdehyde (MDA) and nitrite levels in the hippocampus of the Sco group were higher, but the total thiol, superoxide dismutase (SOD) and catalase (CAT) were lower than that of the control group. Pre-treatment using Pio prevented the changes concerning the biochemical criteria. Conclusion: It is suggested that Pio prevents Sco-induced memory deficits by reducing the brain tissues oxidative stress.

\section{P32}

The Effect of Curcumin on Epilepsy Disorder Treatment: Systematic Review

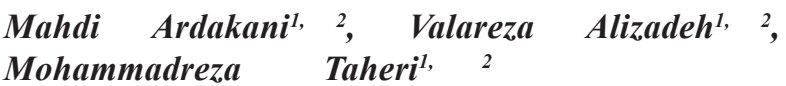

${ }^{1}$ Islamic Azad University, Mashhad Branch, Mashhad, Iran

${ }^{2}$ Student Research Committee, Islamic Azad University, Mashhad Branch, Mashhad, Iran

The Neuroscience Journal of Shefaye Khatam, 2019; 7(3): P32

Introduction: curcumin is a yellow-orange dye obtained from turmeric, the powdered root of 
CURCUMA longa. Curcumin appears to possess a spectrum of pharmacological properties, due primarily to its inhibitory effects on metabolic enzymes. Epilepsy a disorder characterized by recurrent episodes of paroxysmal brain dysfunction due to a sudden, disorderly, and excessive neuronal discharge. Treatment means that the use of drugs, exercise, etc. to cure a person of an illness. Materials and Methods: We searched PubMed and Google Scholar search engine with acute key words" curcumin" and "epilepsy disorder" and" treatment". As a result, 47 articles were found respectively. All articles were published between 2013 until 2018 and have ethical considerations. After investigating the titles, 24 were excluded and by reading abstracts 11 articles excluded and 6 articles excluded by method investigating. At the end of reading 6 articles was included. Results: two articles of these studies revealed that curcumin has the potential role to prevent harmful effects of arsenic on neurogenesis. The other study shows that curcumin has anti-inflammatory effect due to epilepsy, and reduces neuronal loss hippocampus. One study stated that curcumin supplementation decrease in the expression of pro inflammatory cytokines. But, another article results indicates, oral curcumin treatment had no effect on chronic seizures, possibly because it wasn't at adequate levels on that study. And the last study shows that, the chronic seizure reduced in patients who got curcumin, also nitrite and nitrate levels significantly decreased in their blood sample. Conclusion: Until now in most of the studies on this topic have the low number of patients. And we can get more reliable results, if more studies tend to this field. As a result, I think curcumin can be a neuroprotective agent with strong therapeutic potential in some neurological disorders, especially in epilepsy disorder.

\section{P33}

\section{Lipopolysaccharide Inhibited Long Term Potentiation (LTP) and Synaptic Plasticity in the CA1 Area of the Hippocampal Formation}

Amir Reza Beheshti ${ }^{1}$,Motahareh Rouhi Ardeshiri², Esmaeil Akbari ${ }^{2}$

${ }^{1}$ Faculty of Medicine, Mazandaran University of Medical Sciences, Sari, Iran

${ }^{2}$ Department of Physiology, Faculty of Medicine, Mazandaran University of Medical Sciences, Sari, Iran

The Neuroscience Journal of Shefaye Khatam, 2019; 7(3): P33

Introduction: Neuro-inflammation is a common feature in neurodegenerative disorders leading to the cognitive and learning deficiency especially in elderly people. Lipopolysaccharide (LPS), is a part of the outer membrane of Gram negative bacteria that is extensively apply for inducing inflammation in neural system. LPS mediates its effects through stimulating immune system cells and releasing inflammatory cytokines. The aim of this study was to investigate, the effects of lipopolysaccharide (LPS) on long term potentiation (LTP) in rats. Materials and Methods: two groups of rats were allocated to these experiments $(n=10$ in each). These experimental groups were defined as: (1) control, (2) LPS (1 mg/kg). Afterwards, Long-term potentiation (LTP) from CA1 area of hippocampus was recorded following $100 \mathrm{~Hz}$ high frequency stimulus application to the Schaffer collateral. Results: Our data indicated that the amplitude and slope of field Excitatory PostSynaptic Potential (fEPSP) have reduced after the LPS administration, with regard to the control animals. Conclusion: This investigation suggested that chronic inflammation could impair LTP induction by LPS in rats.

\section{P34}

Grafted Dental Pulp Stem Cells Restore Motor Function and Preclude Cerebellar Neurodegeneration and Neuroinflammation in Rat Model of Cerebellar Ataxia

Mohammadhosein Akhlaghpasand, Abbas Aliaghaei

Department of Cell Biology and Anatomical Sciences, Faculty of Medicine, Shahid Beheshti University of Medical Sciences, Tehran, Iran

The Neuroscience Journal of Shefaye Khatam, 2019; 7(3): P34

Introduction: Cerebellar ataxia (CA) is a form of ataxia that adversely affects the cerebellum. Cell replacement therapy (CRT) has been considered as a potential treatment for neurological disorders. In this report, we investigated the neuro-restorative effects of human dental pulp stem cells (DPSCs) transplantation on rat model of CA induced by 3-acetylpyridine (3-AP). Materials and Methods: In this regard, DPSCs were isolated and phenotypically characterized. Then, experimental ataxia was induced by single injection of 3-AP, and bilateral DPSCs implantation was conducted 3 days after 3-AP injection, followed by expression analysis of a number of apoptotic, autophagic and inflammatory genes as well as vascular endothelial growth factor (VEGF) level, along with assessment of cerebellar neurodegeneration, motor coordination and muscle activity. Results: The findings revealed that grafting of DPSCs in 3-AP model of ataxia decreased the expression levels of several inflammatory, autophagic and apoptotic genes and provoked the upregulation of VEGF in the cerebellar region, prevented the degeneration of Purkinje cells against 3-AP toxicity and improved motor coordination and muscle activity. Conclusion: In conclusion, these data indicates in vivo efficacy of DPSCs in the reestablishment of motor skills and reversal of $\mathrm{CA}$.

\section{P35}

Effects of Chamomile Hydroalcoholic Extract on 
Passive Avoidance Memory in Harmaline Induced Tremor Rats

\section{Leila Amini Noghondar, ${ }^{1,2}$, Niloufar Jafari ${ }^{1,2}$, Hassan Abbassian $^{2}$}

${ }^{1}$ Student Research Committee, Faculty of Medicine, Mashhad University of Medical Sciences, Mashhad, Iran

${ }^{2}$ Department of Neurology, Faculty of Medicine, Mashhad University of Medical sciences, Mashhad, Iran

The Neuroscience Journal of Shefaye Khatam, 2019; 7(3): P35

Introduction: Essential tremor (ET) is a progressive neurological impairment and mostly cause unnecessary rhythmic movements mainly in upper extremity. Based on disease severity, its treatment is different and present agents don't show progressive and successful outcome in $25-55 \%$ of cases. Since present treatment protocols have serious side effects, we decided to test the effects of chamomile hydro alcoholic extract on passive avoidance memory in harmaline induced rat model of tremor. Materials and Methods: 40 male rats (weighing 40-60 g) were kept in individual cages with access to food and water ad-libitum in a 12/12 dark/light cycle. The animals divided into 5 groups of 8 , including control saline, Harmaline $10 \mathrm{mg} / \mathrm{kg}$, chamomile 50, 100, $200 \mathrm{mg} / \mathrm{kg}$ IP. Animals were placed in the light arena of the shuttlebox and the door opened and allowed to go to the dark sector without electric shock. When the animal entered the dark sector, an electric pulse was administered to the cage floor $(0.5 \mathrm{~A}, 5 \mathrm{~ms})$. In the retention trials, $24 \mathrm{~h}$ after training, the test was performed to evaluate memory; for this, the animal was placed in the light arena of the shuttle-box apparatus. After $30 \mathrm{~s}$, the door opened and the time required to enter the dark sector was recorded as retention time (step-through latency (STL)). Time in the dark compartment (TDC) was recorded as an indicator of contextual memory. Results: Significant STL improvement was detected through chamomile groups. In addition, more drug dosage caused significant improvement in STL $(34.8 \pm 5.97,59.1 \pm 11.63$, $89.7 \pm 9.14 v s .34 .0 \pm 5.18)$. After Harmaline injection TDC showed significant increase in comparison with control. The more we injected chamomile, greater reduction was detected in TDC $(101.4 \pm 44.38,58.5 \pm 11.68$, 49.4 $\pm 9.58 v$ s. 112.9 \pm 38.65$)$. Conclusion: Although more assessments needed, our results showed chamomile may make place in ET treatment.

\section{P36}

The Mediatory Roles of Erythrocytes Polyunsaturated Fatty Acids in the Relationship Between Dietary Patterns and Depression

Negar Khoshghamat', Gity Sotoudeh ${ }^{2}$, Maryam Khosravi ${ }^{3}$

${ }^{1}$ Student Research Committee, Faculty of Medicine, Mashhad
University of Medical Science, Mashhad, Iran

${ }^{2}$ Department of Community Nutrition, School of Nutritional Sciences and Dietetics, Tehran University of Medical Sciences, Tehran, Iran

${ }^{3}$ Department of Nutrition, Faculty of Medicine, Mashhad University of Medical Sciences, Mashhad, Iran

The Neuroscience Journal of Shefaye Khatam, 2019; 7(3): P36

Introduction: The aim of the study was to examine the association between depression and dietary patterns via the intermediary role of erythrocytes polyunsaturated fatty acids (PUFA). Materials and Methods: In this individually matched case-control observational study, we extracted dietary patterns for 331 persons using factor analysis and assessed erythrocyte PUFAs, using a GC-Mass spectrometry analytical method for 84 people and measured the level of n-3 and n-6 PUFA and some other fatty acids. We diagnosed depression using the criteria of the Diagnostic and Statistical Manual of Mental Disorders and extracted dietary patterns using a valid and reliable semi-quantitative food frequency questionnaire. Results: We observed healthy dietary pattern could relate to the risk of depression $(\mathrm{P}=0.01$ $\&$ until minimum $\mathrm{OR}=0.31,95 \% \mathrm{CI}:(0.14-0.68)$ by the increase of ratio n-3 /n-6 PUFA $(\mathrm{p} \leq 0.03)$ and decrease of ratio $n-6 / n-3$ PUFA ( $\leq \leq 0.005)$. In addition, the unhealthy dietary pattern could associate with the risk of depression $(\mathrm{P}=0.02$ \& until maximum $\mathrm{OR}=2.7$, 95\%CI: (1.25-5.9) via the decreasing of ratio $n-3 / n-6$ PUFA $(p \leq 0.03)$ and increasing of ratio $n-6 / n-3$ PUFA $(p \leq 0.001)$. Principal. Conclusion: Based on our results, the type of dietary pattern via changing in $n-3$ and n-6 PUFA, and the ratio of n-3 to $n-6$ as the mediator variables, relates to the risk of depression. Of course, further studies in this context are necessary.

\section{P37}

Repeated Immunization with BCG Vaccine Induced Cutaneous Thermal Hypersensitivity in Normal Mice

\section{Parisa Sobhani, Majid Hassanpour-Ezatti}

Department of Biology, Faculty of Basic Sciences, Shahed University, Tehran, Iran

The Neuroscience Journal of Shefaye Khatam, 2019; 7(3): P37

Introduction: A significant alleviation in inflammatory visceral pain has been reported after vaccinated against bacillus Calmette-Guerin (BCG) in mice. Accordingly, we investigated whether immunization via repeated vaccination with BCG may effect on the thermal somatic pain threshold in mice? Materials and Methods: Mice $(n=5)$ were immunized by subcutaneously administration of human BCG vaccine $(0.1,1$ and $10 \mathrm{mg} / \mathrm{mice})$ once per week for 9 weeks. At the end of each week, somatic pain threshold of mice was investigated using an acute 
pain model based on the warm water tail withdrawal test. Results: Mice immunized with BCG vaccine showed significant gradual hyperanalgesic response to thermal painful stimulation of the tail during 9th week of treatment, in comparison with before treatment period. The hyperalgesic response was reversed by naloxone pretreatment. Conclusion: The immunotherapy with BCG vaccine induces a secondary adaptive immune response against vaccine antigens that caused a change in somatic thermal pain threshold in mice. It seems that the hyperanalgesic response is related to change in endogenous opioid system function in mice.

\section{P38}

Investigating the Direct and Indirect Impacts of MS on Body Structure and How It Affects the Quality of Life of Patients in Isfahan Province

\section{Alireza Nasr Isfahani, Setareh Samizadeh ${ }^{1}$, Masoumeh Masah ${ }^{2}$ Fereshteh Ashtari ${ }^{3}$}

${ }^{1}$ Student of Biochemistry, Islamic Azad University, Najafabad, Iran

${ }^{2}$ Department of Biochemistry, Islamic Azad University of Najafabad, Esfahan, Iran

${ }^{3}$ Isfahan Neuroscience Research Center, Department of Neurology, Faculty of Medicine, Isfahan University of Medical Silences, Isfahan, Iran

The Neuroscience Journal of Shefaye Khatam, 2019; 7(3): P38

Introduction: Multiple Sclerosis (MS) is an unpredictable degenerative disorder which affects the central nervous system (CNS), specifically the brain and the spinal cord. It involves damages to the fatty myelin sheaths that its task is to protect the nerve fibers and promotes the transmission of nerve impulses symptoms. MS vary widely from person to person and may include sensory disturbances in the limbs, problems with muscle control, tremors muscle stiffness, hyperreflexia, weakness, difficultly walking, poor bladder control and vision problems. Therefore, this study was conducted to investigate and understand the symptoms and complications of the disease in patients with MS. Materials and Methods: This descriptive study was performed on 50 patients among members of the MS society of Isfahan that were selected randomly by convenience sampling. Demographic information including age, gender, marital status and taking the drugs were evaluated. The data collection tools were questioners (MSQOL-54, MSIS-29). The data was analyzed by the software of the statistical community (SPSS, Minitab) after collecting. Results: After data analyzing the results showed that 40.6 percent of patients suffered from relapsing-remitting multiple sclerosis. 31.2percent under 5 years 45.7 percent between 5-10 years and 25.7percent over 10 years, had MS history. Also frequency of patients' hospitalization was 21.6percent once, 10percent twice, and 37.5percent more than twice. The most common symptoms were 70 percent fatigue and weakness, 65percent balance impairment, 55.7percent poor bladder control, 30percent sleep disorder which considered in the progression of the disease in the first and the second year. Conclusion: MS is an autoimmune central nervous system that the most patients are women with ages between 20-40. This disease affects the life quality of patients by slow down or blocking messages between the brain and body.

P39

Alpha Lipoic Acid as an Antioxidant for
Neurodegenerative

Nahid Najafi, Hossein Hosseinzadeh

Department of Pharmacodynamics and Toxicology, Faculty of Pharmacy, Mashhad University of Medical Sciences, Mashhad, Iran

The Neuroscience Journal of Shefaye Khatam, 2019; 7(3): P39

Introduction: Alpha lipoic acid (ALA) is an endogenous antioxidant that interrupts cellular oxidative processes. These properties might qualify ALA for a modulatory role in the treatment of people with neuroinflammation and neurodegenerative disorders. Materials and Methods: We completed a search using SCOPUS database on 27 february 2019 with the following keywords: " $\alpha$-lipoic acid" , " $\alpha$ lipoic acid", "alpha lipoic acid", "Acid alpha-Lipoic", "Thioctic Acid" and Alzheimer, Parkinson, "Multiple Sclerosis" , "cognitive impairment", Neurodegenerative, Neuroinflammation, "Cerebral Injury", "Brain Damage", "Traumatic Brain Injury", "Neurological disorders" and Stroke". Results: The total number of references found after automatically and manually excluding duplicates was 128 references. After primary and secondary screening 92 articles were selected. Regarding to, ALA showed several protective effects against neurodegenerative disorders such as protective effects against $\beta$-amyloid related tissue damage via Alzheimer disease, protecting dopaminergic neuronal cells against oxidative damage in Parkinson disease, inhibition of NF- $\kappa \mathrm{B}$ activation via regulating phosphorylation in ischemic stroke. Conclusion: Generally, ALA leads to the activation of phosphoinositol-3 kinase (PI3K), PKG, and ERK1/2 pathways, which play essential roles in neuronal cell survival. The major protective effects of ALA are mediated throuth the reduction of ROS production, protective effects against of GSH depletion and decrease in mitochondria function. There is a requirement of welldesigned clinical trials to prove the clinical indications of ALA effects for the treatment of neurodegenerative disorders. 
Evaluating the Effect of Sinapis alba Seeds Hydroalcoholic Extract on Passive Avoidance Memory, Motor Coordination and Antioxidant Capacity in Male Adult Mice

Fatemeh Khaledi ${ }^{1}$, Zahra Rezaei ${ }^{1}$, Mohammad Reza Hoijati $^{2}$

${ }^{1}$ Student Research Committee, Shahrekord University of Medical Sciences Shahrekord, Iran

${ }^{2}$ Department of Physiology, Facutly of Medical, Shahrekord University of Medical Sciences, Shahrekord, Iran

The Neuroscience Journal of Shefaye Khatam, 2019; 7(3): P40

Introduction: Most people are using medicinal plants for treating and preventing some diseases. Although many of these medicinal plants have shown positive effects on learning and memory, however there are some other articles that show using medicinal plants cause memory deficit. In the European traditional medicine, Sinapis alba is used to increase memory, but we did not find a scientific article to prove this effect. The aim of this study was to investigate the effect of Sinapis alba extract on passive avoidance memory, motorcoordination and its antioxidant capacity in male mice. Materials and Methods: In this experimental study, 48 male Balb/C mice (25-35 g) were randomly divided into 4 groups of 12 mice. Control group received normal saline (i.p.). Extract treated groups received Sinapis alba extract at doses of 50, 100 and $200 \mathrm{mg} / \mathrm{kg}$ (i.p.). Thirty minutes after injections, passive avoidance memory was measured using Shuttle box and motor-coordination was measured by Rotarod test. Total antioxidant capacity and brain and serum MDA levels were also determined using colorimetric method. Finally, data were analyzed using one-way ANOVA followed by Tukey test. $\mathrm{P}<0.05$ was considered as statistical difference level. Results: Sinapis alba extract at doses of 100 and $200 \mathrm{mg} / \mathrm{kg}$ caused a significant decrease in the step through latency in Shuttle box test $(\mathrm{P}<0.05$ and $\mathrm{P}<0.01$, respectively). Mice receiving Sinapis alba extract at dose of $200 \mathrm{mg} /$ $\mathrm{kg}$ spent less time on Rotarod $(\mathrm{P}<0.05)$ and significant increase in serum and brain MDA levels $(\mathrm{P}<0.5$ and $\mathrm{P}<0.001$, respectively). Conclusion: Sinapis alba extract impairs memory and motor coordination in mice which is probably due to increasing oxidative stress.

\section{P41}

The Association between Serum Calcium and Vitamin D, HTLV-1 Proviral Load, and the Severity of HAM/TSP Clinical Symptoms

Soussan Moussavi', Roya Mallem ${ }^{1}$, Zohreh Vahidi', Saeede Anvari Ardakani, Veda Vakili, Friba Zemorshidi', Houshang Rafatpanah ${ }^{2}$, Reza Boostani ${ }^{3}$

${ }^{1}$ Student Research Committee, Faculty of Medicine, Mashhad University of Medical Sciences, Mashhad, Iran
${ }^{2}$ Inflammation and Inflammatory Diseases Research Center,

Faculty of Medicine, Mashhad University of Medical Sciences, Mashhad, Iran

${ }^{3}$ Department of Neurology, Faculty of Medicine, Mashhad University of Medical Sciences, Mashhad, Iran

${ }^{4}$ Department of Community Medicine, Faculty of Medicine, Mashhad University of Medical Sciences, Mashhad, Iran

The Neuroscience Journal of Shefaye Khatam, 2019; 7(3): P41

Introduction: Human T-lymphotropic virus 1 (HTLV-1) -associated myelopathy/ tropical spastic paraparesis (HAM/TSP) is a progressive disease of the CNS that causes weakness or paralysis of the legs, lower back pain, and urinary symptoms. HAM/TSP imposes a substantial health burden in Mashhad, Northeast of Iran, where the HTLV-1 infection is endemic. Since enhancing immunity is one of the treatment strategies, vitamin D (vitD), as an immunomodulator, might have some effects on HAM/ TSP clinical symptoms. Our study aims to investigate the association between HTLV-1 proviral load, serum calcium and vitD levels, and the severity of HAM/ TSP clinical symptoms. Materials and Methods: In this cross-sectional study, data were collected from 41 HAM/TSP with positive serum anti-HTLV-1 antibody who referred to the Ghaem Hospital Neurology ward, affiliated to Mashhad University of Medical Sciences, Mashhad, Iran, from 2014 to 2017. We investigated the association between laboratory findings and clinical symptoms, including motor disability, the severity of spasticity, and urinary disorders. Results: Subjects without urinary problems had higher mean vitD levels $(p=0.04)$. Except for urinary incontinence, vitD was not significantly associated with the severity of HAM/TSP clinical symptoms $(p>0.05)$. There was no significant association between proviral load, serum calcium level and HAM/TSP clinical symptoms $(p>0.05)$. HTLV1 proviral load and serum calcium $(p=0.2)$ and vitD levels $(p=0.09)$ were not notably associated, as well. Conclusions: The current study illustrates that there was a significant association between one of the HAM/ TSP clinical symptoms and serum levels of vitD.

\section{P42}

Risk of Autism Associated with Prenatal Exposure to Anesthetics

\section{Niloofar Nezaminia, Arefeh Kadkhodaei}

Student Research Committee, Shahrekord University of Medical Sciences, Shahrekord, Iran

The Neuroscience Journal of Shefaye Khatam, 2019; 7(3): P42

Introduction: Autism spectrum disorder(ASD) which is defined as an impairment in social functions and interaction is considered as a developmental 
disorder with symptoms appearing mostly in the first 2 years of life. Symptoms may include difficulty of communication, decrease in the inability to function in social environments, and different sensory input as compared to normal. Prenatal Exposures to certain drugs and medications have been previously found to increase the risk of developmental and behavioral dysfunctions. With the increasing rate of $\mathrm{c}$ sections and in turn the use of anesthetics in the country, it is important to evaluate how significantly associated autism and anesthetics are. Materials and Methods: Databases of PubMed, ScienceDirect and Scopus were searched up to April 2019 for the MeSH terms ।" Autisml", ।" Prenatal Exposurel" and" Anesthesia" independently by two individuals. Results: From a total of 382 referrals, 26 articles and books were retrieved based on the criteria. Studies on animals exposed to anesthetics before birth have shown a decrease in memory, cognition and overall function of the brain. This decrease is caused through a mechanism in which NMDA receptor agonists and GABA receptor antagonists trigger cell death by translocation of mitochondrial membrane proteins such as Bax which sequentially activates caspase-3. With the majority of anesthetics containing at least one of these agents, these findings could be interpreted as an indication of the mentioned association. Clinical cohort studies conducted across the world on human subjects have also been affirmative to these results despite a few that have found no association at all. Furthermore, newborns delivered by $\mathrm{C}$-section with general or regional anesthesia have shown a higher incidence of autism with a risk of 1.52. Conclusion: The majority of studies indicate that newborns exposed to anesthetic agents are at a higher risk of subsequently being diagnosed with ASD, however, future investigations are required due to some studies opposed to the above. Natural vaginal delivery is recommended to avoid this risk, unless medically contraindicated, and therefore, educating mothers in the country is a requirement. Moreover, regional anesthesia is a better substitution for general anesthesia, in case of NVD contraindication due to the less associated risk.

\section{P43}

\section{Vitamin D Deficiency Mediates the Relationship between Dietary Patterns and Depression: A Case- Control Study}

\footnotetext{
Negar Khoshghamat ${ }^{1}$ Gity Sotoudeh ${ }^{2}$, Maryam $^{2}$ Khosravi ${ }^{3}$

${ }^{1}$ Student Research Committee, Faculty of Medicine, Mashhad University of Medical Sciences, Mashhad, Iran

${ }^{2}$ Department of Community Nutrition, Faculty of Nutritional Sciences and Dietetics, Tehran University of Medical Sciences, Tehran, Iran

${ }^{3}$ Department of Nutrition, Faculty of Medicine, Mashhad
}

University of Medical Sciences, Mashhad, Iran

The Neuroscience Journal of Shefaye Khatam, 2019; 7(3): P43

Introduction: Depression, one of the most common psychological conditions, is a major contributor to disability-adjusted life years lost in the world. Due to the complex interactions between nutrients and foods, nutritional epidemiologists have suggested the use of dietary pattern approach in investigating diet-disease relations. Therefore, the aim of present study was to identify dietary patterns in relation with depression, also investigating if some biomarkers mediated this association. Materials and Methods: In this individually matched case-control observational study, 330 depressed and healthy subjects were recruited; Patients were diagnosed by psychiatrists as major depressive disorder, using DSM-IV. Dietary intakes of the subjects in the last 12 months were assessed using a semi-quantitative food frequency questionnaire. Some of all depressed and healthy individuals were chosen randomly for the measurement of serum level $25(\mathrm{OH})$ $\mathrm{D}$, zinc, magnesium and total Antioxidant Capacity (TAC). While evaluating the relationship between dietary patterns and depression, the biomarkers are considered as potential mediator variable. Results: we observed a negative significant relationship between depression and serum vitamin $\mathrm{D}$, whereas there were no significant association between depression and serum level TAC, serum zinc and magnesium. We also identified two healthy and unhealthy dietary patterns led to significantly lower and higher odds for depression, respectively. In mediated analysis, healthy dietary pattern is related to decreased depression via increasing serum vitamin $\mathrm{D}$; while, the unhealthy dietary pattern is related to increased depression via decreasing serum vitamin D. Conclusion: Vitamin D deficiency mediates the relationship between Dietary Patterns and Depression, but further prospective studies are required.

\section{P44}

A Golden Differential Mathematical Model for Prognosis and Treatment Strategy Choosing of ALS and TBI

\section{Afarin Rabbani ${ }^{1}$, Hojjat Shadman Zahroodi ${ }^{2,3}$ \\ ${ }^{1}$ Student Research Committee, Faculty of Nursing and Midwifery, Mashhad University of Medical Sciences, Mashhad, Iran \\ ${ }^{2}$ Student Research Committee, Faculty of Medicine, Mashhad University of Medical Sciences, Mashhad, Iran \\ ${ }^{3}$ Department of Neurology, Faculty of Medicine, Mashhad University of Medical Sciences, Mashhad, Iran,}

The Neuroscience Journal of Shefaye Khatam, 2019; 7(3): P44

Introduction: Neuroinflammation is established as a mediator in the pathogenesis of a variety of disorders such as amyotrophic lateral sclerosis (ALS) and 
traumatic brain injury (TBI). ALS is neurodegenerative disease that selectively degenerates motor neurons. Although there are some therapeutic strategies on ALS progression, but there is no known certain cure for this disease. TBI is a form of acquired damage of the brain and it affects the lives of estimated 10 million people annually around the world. By using a suitable mathematical model, we can predict the treatment and prognose the disease. In this study, we desire to discover a formula for these uses. Materials and Methods: In this analytic original article first we searched about some previous analytic studies which had presented some mathematical models in pathogenesis, treatment and prediction of the neuroinflammation modulated diseases specially ALS and TBI by searching in four databases( Cochrane, PubMed, Medline and Scopus), based on the relevant medical subject headings (MeSH) of: mathematical model, neuroinflammation, ALS, TBI, and adding time filter (last 5 years) and article type filter(clinical trial, meta analyses, systematic reviews) next, we extracted related mathematical models, used ordinary differential equation( ODE) and eliminated similar parameters, so a general effective model was achieved. Results: As the result of search we found 1980 related paper that by using filters of time and type of article, it's limited to 47 . By reviewing the text of articles as the result we achieved 2 main formulas and 12 guide and then after lots of ODEs the gold formula was discovered. Conclusion: We achieved a golden formula to predict the outcome of the disease and the treatment method, which consists of parameters such as IL4 and IL6. Further studies are needed to demonstrate the effectiveness of this formula and other aspects of these diseases.

\section{P45}

Role of GABAB Receptor of the Pedunculopontine Tegmental (PPT) Nucleus on Cardiovascular Responses in Normotensive Rats

\section{Mohammad Hosein Khajavi Rad ${ }^{1,2}$, Hosein Pasandi', Mohammad Naser Shafei ${ }^{4}$, Abolfazl Khajavi Rad}

${ }^{1}$ Student Research Committee, Faculty of Medicine, Mashhad University of Medical Sciences, Mashhad, Iran

${ }^{2}$ Deartment of Neurosciences, Faculty of Medicine, Mashhad University of Medical Sciences, Mashhad, Iran

${ }^{3}$ Deartment of Physiology, Faculty of Medicine, Mashhad University of Medical Sciences, Mashhad, Iran

${ }^{4}$ Division of Neurocognitive Sciences, Psychiatry and Behavioral Sciences Research Center, Mashhad University of Medical Sciences, Mashhad, Iran

${ }^{5}$ Neurogenic Inflammation Research Center, Mashhad University of Medical Sciences, Mashhad, Iran

The Neuroscience Journal of Shefaye Khatam, 2019; 7(3): P45

Introduction: The Pedunculopontine Tegmental (PPT) is a mesencephalic nucleus that involved in numerous functions including movements, sleep, pain and also respiratory and cardiovascular regulation. This nucleus has many neurotransmitters including gamma aminobutyric acid (GABA). In our previous study, the effect of GABAA receptor of the PPT on cardiovascular function was determined. In this study the role of the GABAB receptor of this nucleus on cardiovascular response has been studied. Materials and Methods: In this study animals were randomly divided in following groups: 1) Control and 2-4) Baclofen (as a GABAB agonist with 3 doses; 0.5, 1 and $2.5 \mathrm{~nm}$ ), 5-7) Phaclofen (as a GABAB antagonist with 3 doses; 0.5, 1 and 2.5 $\mathrm{nm})$. To measure the cardiovascular parameters, the femoral artery was cannulated and connected to pressure transducer and by means of a power lab device the mean arterial pressure (MAP), systolic blood pressure (SBP) and heart rate (HR) were recorded. The drugs were microinjected into the PPT nucleus using stereotactic device. Changes of SBP, MAP and HR before and after injection of drugs were calculated and compared with the control group (one-way ANOVA with post hoc tukey's). Results: Microinjection of baclofen and phaclofen at 0.5 and $1 \mathrm{~nm}$ doses did not significantly change the MAP, SBP and HR. However, dose 2.5 baclofen (GABAB agonist), significantly reduced HR $(\mathrm{P}<0.05)$ and had not significant effect on SBP and MAP. The dose 2.5 phaclofen (GABAB antagonist) also significantly increased SBP $(\mathrm{P}<0.05)$ and $\mathrm{HR}(\mathrm{P}<0.01)$ but had not significant effect on MAP. Conclusions: The results show that the GABAB receptor agonist in higher dose has inhibitory effects on cardiovascular parameters, especially on HR.

\section{P46}

\section{Acoustic Analysis of Voice in Parkinson Disease}

Fatemeh Azhdarzadeh ${ }^{1}$ Majid Soltani ${ }^{1}$, Negin Moradi $^{1}$, Davood Kashipazha ${ }^{2}$, Maryam Dastoorpoor ${ }^{3}$

${ }^{1}$ Musculoskeletal Rehabilitation Research Center, Ahvaz Jundishapur University of Medical Sciences, Ahvaz, Iran ${ }^{2}$ Department of Neurology, Faculty of Medicine, Ahvaz Jundishapur University of Medical Sciences, Ahvaz, Iran ${ }^{3}$ Department of Epidemiology and Biostatistics, Faculty of Public Health, Ahvaz Jundishapur University of Medical Sciences, Ahvaz, Iran

The Neuroscience Journal of Shefaye Khatam, 2019; 7(3): P46

Introduction: Acoustic analysis of speech is a powerful, noninvasive, and cost effective tool to study different aspects of motor speech disorders such as the dysarthria associated with Parkinson disease. This motor speech disorders resulted in mono loudness, mono pitch, hoarseness, harshness, breathiness, consonant imprecision and vowel centralization. $\mathrm{f} 2 \mathrm{i} / \mathrm{f} 2 \mathrm{u}$ is an acoustic - articulatory scale that can be an appropriate 
instrument to distinguish dysarthric and normal speech. Materials and Methods: In this study, 40 individuals of Parkinson's disease and 40 healthy individuals have been investigated. PD group was selected by an available sampling method by the diagnosis of neurologist and type of dysarthria was diagnosed with Duffy's protocol by two expertise people in motor speech disorders. Normal group was matched with age and sex with PD group. The task was repeating sentences including /i/ and /u/. Results: Individual with mean age $63 \pm 5.1$ participated in this voice evaluation. Statistics analysis showed difference in $\mathrm{f} 2 \mathrm{i} / \mathrm{f} 2 \mathrm{u}$ between dysarthria and normal group ( $p$ value $<0.001$ ). Conclusion: According to the nature of Parkinson disease and hypokinetic dysarthria vowel formant frequency going to be changed. One of the cost effective and noninvasive procedure to evaluate speech is computing $\mathrm{f} 2 \mathrm{i} / \mathrm{f} 2 \mathrm{u}$. this parameter can help early detection of dysarthtria and prevent its progress; and also help these people to improve their quality of life.

\section{P47}

Ziziphus Jujuba Extract Prevents from Brain Tissues Oxidative Damage and Learning and Memory Impairments of Juvenile Hypothyroid Rats

Zahra Niyazi Mashhadi', Farimah Beheshti', Zahra Baradaran $^{1}$, Mahmoud Hosseini', Hassan AzhdariZarmehri $^{1}$, Masoumeh Gholami ${ }^{1}$, Faranak Jafari ${ }^{1}$

${ }^{1}$ Neuroscience Research Center, Torbat Heydariyeh University of Medical Sciences, Torbat Heydariyeh, Iran

${ }^{2}$ Division of Neurocognitive Sciences, Psychiatry and Behavioral Sciences Research Center, Mashhad University of Medical Sciences, Mashhad, Iran

The Neuroscience Journal of Shefaye Khatam, 2019; 7(3): P47

Introduction: The effects of Ziziphus jujuba (ZJ) extract on brain tissues oxidative damage and learning and memory impairments during neonatal and juvenile growth in hypothyroid rats was examined. Materials and Methods: Thirty pregnant rats after delivery were randomly divided as following groups: (1) control, (2) PTU (propylthiouracil), (3) PTU-ZJ100mg, (4) PTUZJ150mg and (5) PTU-ZJ200mg. All dams except the control group received $0.005 \%$ PTU in their drinking water during lactation. Besides PTU, dams in groups 3,4 and 5 received 100,150 and $200 \mathrm{mg} / \mathrm{kg} \mathrm{ZJ}$ extract respectively. After lactation period, pups continued to receive same experimental treatment for the first 8 weeks of their life. Then, 10 male offspring of each group were randomly selected for Morris water maze (MMW) test. Finally, the animals were deeply anesthetized and the brain tissues were removed for biochemical measurements. Results: PTU increased the time latency in MWM test, while reduced the time spent in target quadrant. PTU reduced serum thyroxine levels and brain levels of thiol groups, catalase and superoxide dismutase (SOD) activities, and significant elevation in hippocampal MDA. Administration of 150 and $200 \mathrm{mg} /$ $\mathrm{kg}$ ZJ extract reduced the time latency, while increased the time spent in target quadrant compared to the PTU group in MWM test. The serum thyroxine concentrations of the animals treated by all doses of the ZJ extract were higher than that of the PTU group. Higher and medium doses of ZJ extract decreased the MDA concentration in hippocampal tissues, while increased thiol contents and catalase and SOD activities compared to the PTU group. Conclusion: The results of this study demonstrate that the hydroalcoholic extract of ZJ have protective effects on hypothyroidism-associated learning and memory impairment during neonatal and juvenile growth in rats. The effects might be due to the protective effects of $\mathrm{ZJ}$ extract against brain tissues' oxidative damage.

\section{P48}

The Effect of Caffeine on Improving the Quality of Life in Patients with Multiple Sclerosis Referred to the Zahedan MS Society in 2018

Afsoon Dadvar ${ }^{1}$, Hamed Amirifard ${ }^{2}$, Alireza Khosravi, MahnazShahrakipour ${ }^{4}$, Farzane Farajian $^{5}$

${ }^{1}$ Student Scientific Research Center, Department of Neurology Zahedan University of Medical Sciences, Zahedan, Iran

${ }^{2}$ Department of Neurology, Faculty of Medicine, Zahedan University of Medical Sciences, Zahedan, Iran

${ }^{3}$ Clinical Immunology Research Center, Department of Neurology, Zahedan University of Medical Sciences, Zahedan, Iran

${ }^{4}$ Zahedan University of Medical Sciences, Zahedan, Iran

5Department of Pharmacology, University of Medical Sciences, Zahedan, Iran

The Neuroscience Journal of Shefaye Khatam, 2019; 7(3): P48

Introduction: Multiple sclerosis (MS) is the most common nervous system disorder in youth. This sickness have a long-lasting and unspecified nature so it has many effects on life quality due to its numerous disabilities. Caffeine can stimulate central nervous system and improve nervous-muscle coordination; So we have decided to assess the effect of caffeine consumption on life quality in multiple sclerosis patients. Materials and Methods: In this randomized clinical trial study, 30 multiple sclerosis subjects were selected. the assessment method of symptoms improvement is based on before and after caffeine consumption.at first subjects were screened based on the entrance criteria includes age between 15-60, weight above 45, not using the equal dose of caffeine and substances preferred by study. And the exclusion criteria includes allergic reaction and feeling discomfort or getting worse. At the first two weeks the patients got placebo and after that they filled the quality of life questionnaire (SF-26) and the results were recorded. After that they got $200 \mathrm{mg}$ 
caffeine daily and the SF-26 questionnaire were filled in weeks 4,8,12 and the results were recorded. IRCT Code: 2017012332142N1 Results: This study was conducted on 30 patients with MS (23 female and 7 male) with the average age of 38.8. Mean SF-26 score in each 4 stage were respectively $73.5,77.05,77.11,74.83$. the result of analyzed frequent measurement indicated that significant changes were made in their quality of life $(p=0.031)$. Conclusion: According to the present study, there is a significant relation between quality of life and caffeine consumption. Based on two by two comparisons it can be concluded that 12 weeks caffeine consumption has a significant effect on quality of life in patients with multiple sclerosis. More studies with larger sample size is recommended to evaluate the results of this study.

\section{P49}

White Matter Hyper Intensities and Cognitive Decline in Parkinson's Disease

\section{Amin Saberi}

Faculty of Medicine, Mashhad University of Medical Sciences, Mashhad, Iran

The Neuroscience Journal of Shefaye Khatam, 2019; 7(3): P49

Introduction: White Matter Hyperintensities (WMHs) have been associated with the Alzheimer's disease. Cognitive decline is a common non-motor manifestations of Parkinson's disease (PD) with an unclear pathogenesis. In this study, we systematically reviewed the association of $\mathrm{WMH}$ with dementia (PD-D) and mild cognitive impairment (PD-MCI) in PD patients. Materials and Methods: PubMed database was searched for the relevant studies using terms related to $\mathrm{WMH}, \mathrm{PD}$ and cognitive disorders. Data extraction was performed using a pre-specified spreadsheet and Newcastle-Ottawa Scale was used for the quality assessment. Meta-analysis was performed to calculate the pooled odds ratio of PD-D and PD-MCI in patients with WMH. Additionally, the pooled WMH volume in PD-D and PD-MCI patients was compared to cognitively normal PD (PD-CN) patients. Results: Twenty-one studies with 2961 patients comprising of 1548 PD-CN, 668 PD-MCI and 397 PD-D patients were included in this systematic review. Nine out of 12 studies found a positive association between the total WMH and PD-D. The pooled OR of PD-D in patients with WMH was 2.8 (CI95: 1.93 - 3.99, p < $0.05)$. However, the pooled WMH volume in PD-D and PD-CN groups was not significantly different (Hedge's $\mathrm{g}=0.66$, CI95: $-0.13-1.46, \mathrm{p}=0.10)$. Eight out of 11 studies did not show a significant association between total WMH and PD-MCI. Pooled OR of MCI in PD patients with WMH was 2.2 (CI95: 1.62 - 3.04, p < 0.05 ) and there was a small significant difference in the pooled WMH volume between the PD-MCI and
PD-CN groups (Hedge's g = 0.34, CI95: 0.05-0.64, $\mathrm{p}$ $<0.05)$. Conclusion: Similar to AD, WMH seems to contribute to the development of cognitive impairment in PD patients. Further studies are needed to localize these WMHs in cognitively impaired PD patients and to understand the pathogenesis of WMH in the cognitive decline.

P50

Comparison of Effect of Four and Eight Weeks of Water Exercises (Water Therapy) on Increasing Cardiovascular Pulmonary Endurance (VO2Max) in Children with Spinal Cord Injury in Bushehr City

\section{Parviz Farzadinia $^{1}$, Alireza Asadnia ${ }^{2}$, Aria Salehpour ${ }^{2}$}

${ }^{1}$ Department of Biology and Anatomical Sciences, Faculty of Medicine, Bushehr University of Medical Sciences, Bushehr, Iran 2Student Research Committee, Bushehr University of Medical Sciences, Bushehr, Iran

The Neuroscience Journal of Shefaye Khatam, 2019; 7(3): P50

Introduction: The present study compares the effect of four and eight weeks of water exercises (water therapy) on increasing cardiovascular endurance in children under the age of 12 with spinal cord injury in Bushehr. Spinal cord injuries can be considered as one of the most common diseases of the central nervous system. It is the main cause of neurological disabilities. Causes motor-status disabilities in an evolving child there are a number of secondary disorders associated with cerebral palsy that may have a greater impact on the child and his family than spinal cord injury, which is the reason why exercise and water exercises (water therapy) are one of the most effective ways to reduce fatigue especially in children with paralysis. Materials and Methods: This quasi-experimental study was performed on 40 children with spinal cord injury. Patients were divided into two groups (one and two) of 20 persons. The group first and second group performed specific exercises in water for four and eight weeks, three days per week and one hour per day, respectively. Parameters of pulmonary cardiovascular endurance (VO2Max) were evaluated on the first, twenty-eighth and fifty-six days using descriptive statistics, oneway sampling, T-test, covariance analysis and paired t-test for moderate averages using SPSS18 software. Results: Surveys showed that patients who had regular water exercise training for four weeks had no effect on the parameters of increasing their cardiovascular pulmonary endurance (VO2Max) compared to those who performed exercises for 8 weeks. Conclusion The individuals, although they tended to spend more time without fatigue, but the results showed that shortterm exercise in water did not increase cardiovascular endurance (VO2Max) compared to the long-term group that performed exercises for eight weeks. 


\section{P51}

Effects of Methylprednisolone on Nerve Regeneration after Delayed Repair in Wistar Rats

Samira Bolandghamat, Morteza Behnam Rassouli, Nasser Mahdavi Shahri, Ali Moghimi

Department of Biology, Faculty of Sciences, Ferdowsi University of Mashhad, Mashhad, Iran

The Neuroscience Journal of Shefaye Khatam, 2019; 7(3): P51

Introduction: Functional recovery after delayed nerve repair is poor due to nerve degeneration and muscle atrophy. It is well known that the inflammation plays a pivotal role in the degeneration process. The aim of this study was to investigate the effects of methylprednisolone (MP) as an anti-inflammatory agent on nerve regeneration after delayed repair in rats. Materials and Methods: The sciatic nerve of male Wistar rats was transected at the mid-thigh and the operated animals were administrated a low dose of methylprednisolone succinate $(2 \mathrm{mg} / \mathrm{kg} / \mathrm{day}$, i.p.) for a period of 2 weeks. The control animals underwent the same procedure without treatment. After a period of 4-weeks, the transected nerve was re-exposed, refreshed and the nerve gap was repaired by a $6 \mathrm{~mm}$ allograft from the sciatic nerve of normal donors. Nineteen weeks after surgical repair, histomorphometric analyses were performed in both graft and distal part of repaired nerves to determine the number of mast cells, nerve fiber density and diameter of axons. Results: Although there was no remarkable difference in the fiber density between the two groups, the mean diameter of axons in the distal stump of MP group was approximately 1.7 times of control. Interestingly, in the MP group, the density of mast cells in the graft was about 2 fold higher than that of the control group. Conclusion: Following nerve transaction, a 2 week systemic administration of MP resulted in an increase in axonal diameter. This finding shows that, in situations that immediate nerve repair using surgical intervention is not available, methylprednisolone administration may keep the regenerative capacity in the distal stump of transected nerve. On the other hand, the increased density of mast cells in the graft is in contrast to immunosuppressant effects of MP in the transplantational approches and needs to further studies.

\section{P52}

Protective Effects of Selenium Against Depression and Anxiety Like Behaviors Induced by Lipopolysaccharide in Rats

Mostafa Mansouri', Mohammad Mahdi Sotoudeh', Farimah Beheshti ${ }^{3}$, Mahmoud Hosseini ${ }^{1}$, Hamid Reza Sadeghnia ${ }^{4}$, Ali Shamshirian ${ }^{2}$

${ }^{1}$ Division of Neurocognitive Sciences, Psychiatry and Behavioral
Sciences Research Center, Mashhad University of Medical Sciences, Mashhad, Iran

${ }^{2}$ Neurogenic Inflammation Research Center, Faculty of Medicine, Mashhad University of Medical Sciences, Mashhad, Iran

${ }^{3}$ Neuroscience Research Center, Torbat Heydariyeh University of Medical Sciences, Torbat Heydariyeh, Iran

${ }^{4}$ Pharmacological Research Center of Medicinal Plants, Mashhad University of Medical Sciences, Mashhad, Iran

The Neuroscience Journal of Shefaye Khatam, 2019; 7(3): P52

Introduction: The effects of selenium in a rat model of lipopolysaccharide (LPS) induced anxiety and depression like behaviors was challenged. Materials and Methods: The animals were allocated into 4 groups ( $n=8-9$ in each) and treated by: (1) saline as a control group, (2) LPS 1 $\mathrm{mg} / \mathrm{kg}$, was injected two hours before behavioral tests, (34) Selenium 100 or $200 \mu \mathrm{g} / \mathrm{kg}$ before LPS. Open- field test (OFT), elevated plus maze test (EPT) and forced swimming (FS) test were done. The brains were then collected to examine oxidative stress criteria. Results: LPS increased the immobility while increased active and climbing time $(\mathrm{P}<0.001)$ in FS test. Selenium improved performances of the rats in FS test $(\mathrm{P}<0.001)$. In EPT, LPS decreased the entry into and the time spent in the open arms while, increased the time spent in the closed arms $(\mathrm{P}<0.05-\mathrm{P}<0.01)$. The higher dose of selenium increased the entry into and the time spent in the open arms while, decreased the time spent in the closed arms $(\mathrm{P}<0.01-\mathrm{P}<0.01)$. In OFT, LPS decreased the crossing, the time spent and the traveled distance in central zone and also total crossing and total distance compared with control $(\mathrm{P}<0.01$ - $\mathrm{P}<0.001)$. The higher dose of selenium improved performances of the rats in OFT $(\mathrm{P}<0.05-\mathrm{P}<0.01)$. LPS injection also increased MDA while decreased thiol, SOD and CAT $(\mathrm{P}<0.001)$. Selenium protected from brain tissues oxidative damage $(\mathrm{P}<0.01-\mathrm{P}<0.001)$. Conclusion: It was demonstrated that administration of LPS induced depression and anxiety like behaviors in rats which was reflected by impaired performances of the rats in FST, $\mathrm{OF}$ and EPM. Selenium improved performances of the rats in behavioral tests. Therefore, a protective effect for selenium against depression and anxiety like behaviors induced by LPS is suggested

\section{P53}

Evaluating the Association of Trigeminal Neuralgia and Diabetes Mellitus: A Retrospective Study

\section{Zahra Ranjbar', Fatemeh Lavaee ${ }^{1}$, Ali Mohammad Shahim $^{2}$}

${ }^{1}$ Oral and Dental Disease Research Center, Department of Oral and Maxillofacial Disease, Faculty of Dentistry, Shiraz University of Medical Sciences, Shiraz, Iran

${ }^{2}$ Student Research Committee, Faculty of Dentistry, Shiraz University of Medical Sciences, Shiraz, Iran

The Neuroscience Journal of Shefaye Khatam, 2019; 7(3): P53 
Introduction: this study is designed to evaluate the relationship between diabetes mellitus (DM) and trigeminal neuralgia $(\mathrm{TN})$, in order to provide new insight for TN pathogenesis. Materials and Methods: This retrospective cross sectional study compared the prevalence of DM in patients with trigeminal neuralgia and healthy patients without TN during 2007-2018. In both TN patients and control group, 193 participants were enrolled. These participants were the patients who referred to the Oral and Maxillofacial Medicine Department of the Dentistry School of Shiraz University of Medical Sciences. The age and sex of both groups were matched. Diabetes mellitus (type 1 and 2) was diagnosed based on their reports and routine requested lab test (fasting blood sugar, 2 hours post prandial). Odds ratio and chi square test were used to compare mean values. Results: The prevalence of DM in TN patients and Control group was $11.4 \%$ and $9.8 \%$. The mean age of participants were $56.96 \pm 13.66$ and $56.46 \pm 13.47$ in TN patients and Control group. The difference of TN prevalence between both groups was not significantly different $(\mathrm{P}-$ value $=0.62)$. Conclusion: Although the prevalence of DM in patients with TN was higher than control group, the difference was not significantly different ( $\mathrm{P}$ - value $>0.05$ ). Evaluated women with TN, expressed DM 25\% more than men affected by TN.

\section{P54}

Effectiveness of Progressive Muscle Relaxation Protocol on Parkinson's Disorder: Acase Report

\section{Sahar Sadat Nazm Bojnourdi}

Department of Cognitive Sciences, Cognitive Psychology, Faculty of Psychology and Educational Sciences, Ferdowsi University of Medical Sciences, Mashhad, Iran

The Neuroscience Journal of Shefaye Khatam, 2019; 7(3): P54

Introduction: Parkinson's disease is a progressive disorder of motor and non-motor skills. Therapeutic management for patients is also important for drug therapy and rehabilitation interventions, but the important thing is that psychological and psychological aspects of motor and neurological problems should be considered. Attitudes toward disease, face-to-face with hidden anxiety in unconscious, sleep problems can indirectly affect the development of posture in the development of negative symptoms of muscle tone, posture. In addition, relaxation-based approaches have had a positive effect on the improvement of anxiety and depression symptoms. In this study, a combination of progressive muscle relaxation exercises (Jakobson protocols), mental retardation, mental imaging, and occupational therapy exercises have been studied. The purpose of this study was to evaluate the efficacy of Jacobson muscle relaxant tone protocol in the treatment of Parkinson's motor disorders. In this research, the single-subject project was used with the base line and 3 months' follow-up. The patient was a 55-year-old man with Parkinson's disease. Beck's anxiety and depression questionnaire and a stress assessment questionnaire, feedback on Clenro's disease, and the treatment process were used in this study. The results showed that the patient received a significant clinical change in treatment measures. The progressive pacing of Parkinson's, anxiety and depression symptoms and attitudes toward disease are effective. Breakdown and weekly showed that the amount of anxiety decreased during the daily activity of the patient. Treatment outcomes continued for 3 months. Conclusion: The combination of relaxation, muscle tension and respiratory training improves the patient's symptoms. Patients with Parkinson's disease may also benefit from mental health problems.

\section{P55}

Investigating the Impact of Electromyographic Biofeedback on Improvement of Hand Grasp Function and EEG Signal Dynamics of Hemiplegic Stroke Patients

\section{Mohammad Ebrahim Hashemi ${ }^{1}$, Hamid Reza Kobravi $^{1}$ Alireza Alehashemi ${ }^{2}$}

${ }^{1}$ Research Center of Biomedical Engineering, Mashhad Branch, Islamic Azad University, Mashhad, Iran

${ }^{2}$ Department of Neurology, Mashhad Branch, Islamic Azad University, Mashhad, Iran

The Neuroscience Journal of Shefaye Khatam, 2019; 7(3): P55

Introduction: After cardiovascular disease and cancers, stroke is the third leading cause of death in the world and the second most common death reason in Iran. Recovery through pharmaceutical and physiotherapy methods is very time-consuming and in some cases, it is not achievable. Hence, an effective, low-cost, and noninvasive treatment is very important for stroke patients. One of these applied techniques is biofeedback therapy. Materials and Methods: The purpose of this study was to investigate the effect of electromyography biofeedback therapy to improve the hand grasping function of stroke patients. In addition, time-frequency features of electroencephalography (EEG) signals of patients were investigated in all frequency bands. Results: Results indicate that after the implementation of the biofeedback intervention, the ability of patients to control the position of the fingers has improved significantly and the phenomenon of joint locking of fingers is no longer occurred. In addition, it was observed that there was a significant difference between the time and frequency characteristics of brain signals before and after the biofeedback intervention. But the change in the value of the features in the Beta and Gamma frequency bands has been higher than in other frequency bands. These results could mean the activation of some neuronal networks 
that were deactivated by the stroke. In fact, these neural networks were activated by biofeedback-based exercise, and consequently, the processes of motor cortex commands have been restored. Conclusion: In this research, the effectiveness of an intervention method was studied based on electromyography biofeedback to improve fingers control in chronic stroke patients. Overall, the results show that the motor exercise based on bio feedback electromyography could lead to improve the motor abilities and controllability of the patients' hands.

\section{P56}

Effects of Cannabinoid Receptor Modulation on First Spike Latency in Rat Cerebellar Purkinje Cells Via Whole Cell Recording Under Harmaline Toxicity

\section{Arash Khojasteh Zonoozi ${ }^{1}$, Fateme Sadat Abolghasemi ${ }^{1}$, Benjamin Jason Whalley ${ }^{2}$, Mohammad Shabani, Hassan Abbassian ${ }^{4}$}

\begin{abstract}
'Student Research Committee, Faculty of Medicine, Mashhad University of Medical Sciences, Mashhad, Iran

${ }^{2}$ Departement of Pharmacology, Faculty of Chemistry, Food and Nutritional Sciences and Pharmacy, University of Reading, Reading, UK

${ }^{3}$ Kerman Neuroscience Research Center, Neuropharmacology Institute, Kerman University of Medical Sciences, Kerman, Iran ${ }^{4}$ Department of Neuroscience, Faculty of Medicine, Mashhad University of Medical Sciences, Mashhad, Iran
\end{abstract}

The Neuroscience Journal of Shefaye Khatam, 2019; 7(3): P56

Introduction: Essential tremor (ET) is a neurological disease affecting about $1 \%$ worldwide with a considerable impact on quality of life. This condition is linked to the purkinje cells of cerebellum which can be stimulated with harmaline via climbing fibers stemming from inferior olive. Additionally, cannabinoids effects on different parts of CNS including purkinje cells are an advert subject for researchers. As a result, we aimed to focus on cannabinoid receptors modulation on first spike latency pattern in cerebellar purkinje cells affected by harmaline as an ET simulator. Materials and Methods: Four groups of forty to sixty gram weighted rats were randomly collected; two control groups injected with normal saline and harmaline each apart, and two other harmaline-injected groups with parallel administration of $1 \mathrm{mg} / \mathrm{kg}$ cannabinoid agonist and antagonist, WIN 55,212-2 (WIN) and AM251 respectively. First spike latency response to five levels of positive and negative evoked charges from 0.1 to $0.5 \mathrm{nA}$ was measured by whole cell recording provided by Digidata 1440 and its software (Pclamp 10.1). The data was analyzed by Prism 6. Results: In most positive evoking charges, AM251 significantly propelled the first spike latency pattern to the control group with median of $0.05 \mathrm{~s}$ delay. This substance did not represent the same results in negative charges remarkably (between $0.04 \mathrm{~s}$ and $0.2 \mathrm{~s}$ delay). While the positive charges in WIN-treated groups resulted in a wide range of delaying times, the negative induced charges brought up some short timeconsuming first spikes. Almost none of WIN patterns demonstrates specific correlation between neither control nor harmaline groups. Conclusion: Considering the observed effects of cannabinoid antagonist on first spike latency pattern of purkinje cells administrated with harmaline, AM251 can be contemplated as a substance reducing the effect of harmaline toxicity which our other whole cell recording data can support its role.

\section{P57} Auraptene Induces Apoptosis in Human Malignant
Glioblastoma Cell Line Through Reactive Oxygen
Species (ROS)

Abolfazl Maghrouni ${ }^{1}$, Mohammad Jalili-Nik ${ }^{2}$, Hamed Sabri', Sajjad Javanshir ${ }^{3}$, Amir Reza Afshari ${ }^{4,5}$

${ }^{1}$ Department of Medical Genetics, Faculty of Medicine, Tehran University of Medical Sciences, Tehran, Iran

${ }^{2}$ Department of Medical Biochemistry, Faculty of Medicine, Mashhad University of Medical Sciences, Mashhad, Iran

${ }^{3}$ Faculty of Dentistry, Mashhad University of Medical Sciences, Mashhad, Iran

${ }^{4}$ Department of Pharmacology, Faculty of Medicine, Mashhad University of Medical Sciences, Mashhad, Iran

${ }^{5}$ Pharmacological Research Center of Medicinal Plants, Mashhad University of Medical Sciences, Mashhad, Iran

The Neuroscience Journal of Shefaye Khatam, 2019; 7(3): P57

Introduction: Glioblastoma multiforme (GBM) is the most aggressive type of brain tumor in adults, and represent one of the most challenging cancers to control due to its aggressive nature. This study aimed to investigate the cytotoxic effects of a natural product, Auraptene, and the signaling pathways affected in a human malignant GBM (U87) cells. Materials and Methods: The Auraptene-induced ROS production in U87 cells evaluated by use of a cellular reactive oxygen species detection method. Changes of mRNA level of some essential genes involved in apoptosis were also evaluated in Auraptene-treated cells. Results: Our results demonstrated a significant decline in ROS production in the first 2, and 6 hours after treatment with Auraptene, however, ROS level increased in other incubation periods ( 8 and 24 hours). Interestingly, $\mathrm{N}$-acetyl cysteine (NAC) markedly attenuated the Auraptene-induced ROS production and reversed Auraptene-induced cytotoxicity in 8 and 24 hours after treatment as well. Induction of apoptosis occurred in the first 24 and 48 hours in a concentration- and timedependent manner. The qRT-PCR results indicated an up-regulation in p21, CXCL3 and a down-regulation in Cyclin D1 mRNA expression. Conclusion: In general, 
this study showed an increase in ROS level is at least one of the mechanisms associated with Auraptene-induced toxicity on glioma cells. Also, the induction of apoptosis through $\mathrm{Bax} / \mathrm{Bcl}-2$ regulation and expression of genes involved in apoptosis initiation are other mechanisms by which Auraptene instigates its cytotoxic effects in U87 cells. Therefore, Auraptene could be utilized as a potential novel anti-GBM agent, after complementary studies.

\section{P58}

Effects of Moderate Treadmill Exercise and Fluoxetine on Mrna Expression of Apoptosis Related Protein in A Rat Model of Post-Traumatic Stress Disorder

Zahra Hosseini Bajestani', Pouya Darzi', Reza Yakhkeshi $^{1}$, Fatemeh Roshani, Sara Shafia $^{5}$

${ }^{1}$ Student Research Committee, Mazandaran University of Medical Sciences, Sari, Iran

${ }^{2}$ Department of Physiology, Mazandaran University of Medical Sciences, Sari, Iran

The Neuroscience Journal of Shefaye Khatam, 2019; 7(3): P58

Introduction: Post-traumatic stress disorder (PTSD) is a condition that develops after an individual has experienced a major trauma. Currently, selective serotonin reuptake inhibitors (SSRIs) like fluoxetine are the first-line choice in PTSD drug treatment but their moderate response rates and side effects indicate an urgent need for the development of new treatment. Physical activity is known to improve symptoms of certain neuropsychiatric disorders. The present study investigated the effects of moderate treadmill exercise, the antidepressant fluoxetine and the combined treatment on mRNA expression of apoptosis - related proteins in a rat model of PTSD: the single prolonged stress (SPS) model. Materials and Methods: Rats were exposed to SPS (restraint for $2 \mathrm{~h}$, forced swimming for $20 \mathrm{~min}$ and ether anesthesia) and were then kept undisturbed for 14 days. After that, SPS rats were subjected to chronic treatment with fluoxetine $(10 \mathrm{mg} / \mathrm{kg} / \mathrm{day}$, for 4 weeks), moderate treadmill running (4 weeks, 5 day per week) and the combined treatment (fluoxetine plus treadmill exercise), followed by apoptosis markers assessments. Results: This study showed that SPS significantly increased the mRNA expression of the protein Caspase- 3 in the hippocampus, increase in apoptotic factor in hippocampus causing of cell death. We found that all treatments (fluoxetine, exercise and the combined treatment) down regulated Caspase-3 mRNA in the hippocampus, which shifted to the cell survival. Conclusion: The anti-apoptotic effects of the combined treatment (exercise + fluoxetine) were much stronger than those of either treatment alone, suggesting a synergistic effect between them in the hippocampus.
P59

Study of Three Common Mutations in CHRNE and CHAT Genes in Iranian Patients with the Congenital Myasthenic Syndrome

Amirhossein Ashnaei', Zahra Farjami, 2, Negar Khodaeinia $^{2}$, Neshat Ebrahimi ${ }^{3}$ Zeinab Sadat Hosseini ${ }^{4}$

${ }^{1}$ Department of Modern Sciences and Technologies; Faculty of Medicine, Mashhad University of Medical Sciences, Mashhad, Iran

${ }^{2}$ Department of Medical Genetics, National Research Center of Genetic Engineering and Biotechnology (NIGEB), Tehran, Iran ${ }^{3}$ Laboratory of Cedars-Sinai Medical Center, Los Angles, California, USA

${ }^{4}$ Student Research Committee, Mashhad Branch, Islamic Azad University, Mashhad, Iran

The Neuroscience Journal of Shefaye Khatam, 2019; 7(3): P59

Introduction: Congenital myasthenic syndrome (CMS) is the heterogeneous group of inherited disorders, characterized by dysfunction of neuromuscular junction (NMJ) transmission which appears shortly after birth. The patients suffered from CMS showed the resembling clinical manifestations. CMS is genetically inherited and caused by vary of DNA mutation. CMS is also known as mutations expressed in the NMJ such as acetylcholine receptor (AChR) subunits. Materials and Methods: In this study mutations of CHAT gene in exon 5 and CHRNE gene in exon 11 and 12 in 23 patients with congenital myasthenic syndrome were investigated. For all the patients, DNA sequencing and PCR were done. Results: Our investigations detected the homozygous single base pair deletion in exon 12 of the CHRNE gene (chr17:4802186delC). This region is conserved across mammalian evolution which wasn't filed in the 1000 genomes database. The mutation in CHAT gene was nonsense and had no effect on the incidence of the disease. Conclusion: CHRNE variation possibly is classified as a significant variant on etiology of CMS and can be suggested that Iranian CMS population carry regional pathogenic mutations. Notably, it could be revealed via targeted as well as whole genome sequencing.

\section{P60}

Investigation of Relationship between Size and Volume of Thalamus by MRI in Patients with Multiple Sclerosis Disability

Mohammad Ayyobi Nia ${ }^{1}$, Seyed Hossein Vaezzadeh ${ }^{1}$, Hadi Khodadadi', Saeed Naghibi ${ }^{1}$

${ }^{1}$ Department of Medicine, Faculty of Medical Sciences, Azad University of Medical Science, Mashhad, Iran

${ }^{2}$ Department of Physiology, Faculty of Medicine, Mashhad University of Medical Sciences, Mashhad, Iran 
The Neuroscience Journal of Shefaye Khatam, 2019; 7(3): P60

Introduction: Multiple sclerosis is one of the most common neurological disorders and the third cause of neurologic disability in young adults. The disease is classified into those diseases that myelin membrane of nerves is destroyed. Involvement of thalamus in this disease can cause cognitive impairment and motor disabilities and can reduce quality of life. Clinical manifestations include loss of vision, ataxia, anxiety, paresthesia, loss of sensation, weakness, spasticity, ataxia, and bladder dysfunction. Materials and Methods: This study was performed in cross sectional analysis method. 40 patients were enrolled in a non-probabilistic method with informed consent and considering entry criteria. Then, the EDSS questionnaire was completed and the size and volume of thalamus were examined by MRI and the largest axial section was considered. Results were analyzed by SPSS. Results: In this study, the distribution of age and sex among the participants was: 23 women $(57.5 \%)$ and 17 men $(42.5 \%)$ with a mean age of $36.3 \pm 9.3$ as well as, $15(37.5 \%)$ were single and $25(62.5 \%)$ were married. Bachelor's degree and higher $(32.5 \%)$ and housewives $(30 \%)$ were the most frequent. The mean duration of disease was $3.5 \pm 1.4$ years. The results in this study showed that significant reverse linear correlation between thalamus volume and disability $(\mathrm{P}>0.05)$ and thalamus size and disability $(\mathrm{P}>0.05)$ and direct linear relationship Between thalamus size and thalamus volume $(\mathrm{P}>0.05)$. Conclusion: Generally, it can be concluded that in patients with multiple sclerosis, decreasing the size and volume of thalamus increases the incidence of patients' disability.

\section{P61}

\section{Neuroprotective Effects of Drugs Were Used in Diabetes Mellitus}

\section{Maryam Paseban ${ }^{1}$ Masumeh Mirzaee ${ }^{1}$ Saeed Niazmand ${ }^{1, \quad 2}$}

${ }^{1}$ Department of Physiology, Faculty of Medicine, Mashhad University of Medical Sciences, Mashhad, Iran

${ }^{2}$ Neurogenic Inflammation Center, Mashhad University of Medical Sciences, Mashhad, Iran

The Neuroscience Journal of Shefaye Khatam, 2019; 7(3): P61

Introduction: The aim of the current study is investigation of the effect of combined intake of high dose of aspirin, atorvastatin, captopril and metformin on oxidative stress in the brain cortex and hippocampus of streptozotocin (STZ)-induced diabetic rats. Materials and Methods: Rats were randomly divided into the following eleven groups: control and diabetic (D), as well as 9 groups that were treated with metformin (M, $300 \mathrm{mg} / \mathrm{kg}$ ) or aspirin (ASA, $120 \mathrm{mg} / \mathrm{kg}$ ) alone or in different combinations with captopril (C, $50 \mathrm{mg} /$ $\mathrm{kg}$ ), or atorvastatin (AT, $40 \mathrm{mg} / \mathrm{kg})$, as follows: (D+M),
$(\mathrm{D}+\mathrm{ASA}), \quad(\mathrm{D}+\mathrm{M}+\mathrm{ASA}), \quad(\mathrm{D}+\mathrm{M}+\mathrm{C}), \quad(\mathrm{D}+\mathrm{M}+\mathrm{AT})$, $(\mathrm{D}+\mathrm{M}+\mathrm{C}+\mathrm{ASA}), \quad(\mathrm{D}+\mathrm{M}+\mathrm{C}+\mathrm{AT}), \quad(\mathrm{D}+\mathrm{M}+\mathrm{AT}+\mathrm{ASA})$, and $(\mathrm{D}+\mathrm{M}+\mathrm{C}+\mathrm{AT}+\mathrm{ASA})$. The rats in treatment groups daily received drugs by gavage for six weeks. Finally, serum lipid profile and levels of oxidative markers in the brain cortex and hippocampus tissues were evaluated. Results: The level of MDA in cortex and hippocampus in all treated groups significantly decreased $(p<0.05)$. There was significant increase in total thiol concentration as well as catalase activity in treated rats with $\mathrm{M}+\mathrm{AT}, \mathrm{M}+\mathrm{C}+\mathrm{ASA}, \mathrm{M}+\mathrm{C}+\mathrm{AT}, \mathrm{M}+\mathrm{AT}+\mathrm{ASA}$ and $\mathrm{M}+\mathrm{C}+\mathrm{AT}+\mathrm{ASA}$ groups in cortex and hippocampus in comparison with the diabetic rats $(\mathrm{p}<0.05)$. Also, the SOD activity in all treated rats with drugs significantly boosted compared to the diabetic rats $(\mathrm{p}<0.05-\mathrm{p}<0.01)$. Conclusion: Our findings were showed that combined use of high dose of aspirin, metformin, captopril and atorvastatin potentiate their antioxidant effects on the brain and also have improvement effect on the cognitive functions with their neuroprotective effects on hippocampus area.

\section{P62}

Early Diagnosis of Parkinson's Disease with Microarray Analysis Data

Amirabbas Shahsavari', Mohsen Ahmadi', Seyed Mojtaba Mousavi

${ }^{1}$ Cellular and Molecular Research Center, Department of Clinical Laboratory Science, Qom University of Medical Sciences, Qom, Iran

${ }^{2}$ Department of Genetic, Faculty of Medical, Hormozgan University of Medical Sciences, Bandar Abbas, Iran

${ }^{3}$ Department of Parasitology and Mycology, Faculty of Medicine, Tarbiyat Modares University, Tehran, Iran

The Neuroscience Journal of Shefaye Khatam, 2019; 7(3): P62

Intrudction: Parkinson's disease (PD) is the second most common neurodegenerative disease, and it is a multifactorial disease with no definite diagnostic index. The aim of this study is to construct a molecular network to find molecules that play important roles in the progression of PD with analysis the microarray data for early diagnosis and intervention. Materials and Methods: We downloaded two gene expression profiles (GSE54536 and GSE100054) from the Expression Omnibus (GEO) database to analyze possible markers. The Genes were analyzed with GEO2R. There were 1790 and 967 differentially expressed genes (DEGs) in GSE54536 and GSE100054 respectively. A total of 125 genes co-exist in the DEGs of the two data sets. Results: KEGG pathway analysis showed that 125 DEGs were enriched in Aldosterone synthesis and secretion, Gap junctions, Platelet activation, Rap1 signaling pathway, and Estrogen signaling pathway. There were 20 hub genes among 125 DEGs analyzed by PPI that involved 
in Platelet activation, Inflammatory response, Innate immune response, B cell receptor signaling, Stimulatory C-type lectin receptor signaling, Lipopolysaccharide response, Leukocyte migration, and Regulation of cell proliferation. Additionally, 42 differences in miRNAs were found in GSE100054. We constructed a miRNAmRNA regulatory network depicting interactions between the predicted genes and the 125 DEGs. 34 miRNA-mRNA pairs were obtained. We found GNAQ and TMTC2 were the most important mRNAs in the network analyzed by Cytoscape APP centiscape, and their degrees in centiscape 2.2 were all 10 . has-miR-142 was the most important miRNA (the highest degree is 4 in centiscape2.2), which forms miRNA-mRNA pairs with GNAQ, TMTC2, BEND2, and KYNU. Conclusions: This study provides data of potential biomarkers and therapeutic targets for PD diagnosis and treatment. Among them, hsa-miR-142 is a critical miRNA in the PD network, and may be involved in PD progression by regulating GNAQ, TMTC2, BEND2, and KYNU.

\section{P63}

Application of the Adenosine A2A Receptor Antagonist Attenuates Spatial Memory Deficit and Extent of Demyelination Areas in LyolecithinInduced Demyelination Model

\section{Atefeh Akbari', Maryam Ghasemi-Kasman ${ }^{2}$}

${ }^{1}$ Student Research Committee, Babol University of Medical Sciences, Babol, Iran

${ }^{2}$ Cellular and Molecular Biology Research Center, Health Research Institute, Babol University of Medical Sciences, Babol, Iran

The Neuroscience Journal of Shefaye Khatam, 2019; 7(3): P63

Introduction: In recent years, inactivation of $\mathrm{A} 2 \mathrm{~A}$ adenosine receptors has been emerged as a novel strategy for treatment of several neurodegenerative diseases. Although numerous studies have shown the beneficial effects of A2A receptors blockade on spatial memory, the impacts of selective adenosine $\mathrm{A} 2 \mathrm{~A}$ receptors on memory performance has not yet been examined in the context of demyelination. In the present study, we evaluated the effect of A2A receptor antagonist SCH58261 on spatial memory and myelination in an experimental model of focal demyelination in rat fimbria. Materials and Methods: Demyelination was induced by local injection of lysolecithin (LPC) 1\% (2 $\mu \mathrm{l})$ into the hippocampus fimbria. SCH58261 $(20 \mu \mathrm{g} / 0.5 \mu \mathrm{l}$ or $40 \mu \mathrm{g} / 0.5 \mu \mathrm{l})$ was daily injected intracerebroventricularly (i.c.v.) for 10 days' post LPC injection. The Morris water maze test was used to assess the spatial learning and memory on day 6 post lesion. Myelin staining and immunostaining against astrocytes/microglia were carried out 10 days' post LPC injection. Results: The administration of adenosine $\mathrm{A} 2 \mathrm{~A}$ receptor antagonist prevented the spatial memory impairment in LPC receiving animals. Myelin staining revealed that application of SCH58261 reduces the extent of demyelination areas in the fimbria. Furthermore, the level of astrocytes and microglia activation was attenuated following administration of A2A receptor antagonist. Conclusion: Collectively, the results of this study suggest that $\mathrm{A} 2 \mathrm{~A}$ receptor blockade can improve the spatial memory and protect myelin sheath, which might be considered as a novel therapeutic approach for multiple sclerosis disease.

\section{P64}

Resveratrol Attenuates Lipopolysaccharide-Induced Cognitive Impairments in Rats Via up Regulation of GluA1 Subunit of AMPA Receptors

Maryam Talebrohi ${ }^{1}$, Hamideh Taheri ${ }^{1}$, Mehran Hosseini $^{1,}$, 2

${ }^{1}$ Department of Anatomy, Faculty of Medicine, Birjand University of Medical Sciences, Birjand, Iran

${ }^{2}$ Cellular and Molecular Research Center, Department of Anatomy, Faculty of Medicine, Birjand University of Medical Sciences, Birjand, Iran

The Neuroscience Journal of Shefaye Khatam, 2019; 7(3): P64

Introduction: Accumulating evidence suggests that synaptic dysfunction plays a major role in early Alzheimer's disease (AD) pathogenesis prior to neuronal loss. Currently, a number of studies displays therapeutic potential of some natural products like Resveratrol. Previous studies indicate that Resveratrol by suppressing the expression of tumor necrosis factor alpha and Nuclear factor-kappa B, whereas promoting the interleukin-10 exerts anti-inflammatory activities in pathologic conditions specially AD. The aim of the present study was to determine whether resveratrol modulates synaptic function by affecting $\alpha$-amino-3hydroxy-5-methyl-4-isoxazolepropionic acid (AMPA) receptors in the Lipopolysaccharide (LPS)-induced memory impairment as a model for AD. Materials and Methods: Neuroinflammation was induced by intracerebroventricular (ICV) injection of LPS $(50 \mu \mathrm{g} / 20 \mu \mathrm{l} ; 10 \mu \mathrm{l}$ per ventricle) using stereotaxic apparatus in aged male Wistar rats $(n=16)$. A control group $(n=8)$ only received ICV injection of the vehicle solution. The LPS-injected rats divided into model and experiment groups respectively treated with saline and Resveratrol $(30 \mathrm{mg} / \mathrm{kg})$, and control rats that received saline. All the treatments were intraperitoneally, once per day, and lasted for consecutive 14 days. At the 7 th day, the Y-maze test was performed to assess cognitive function. Moreover, on the 14th day, the animals were sacrificed, decapitated, and their hippocampus collected for further molecular assessments. The mRNA expression levels of hippocampal GluA1-4 were assessed using real-time PCR analysis. Results: 
The results of the Y-maze test showed that Resveratrol significantly attenuated memory damage. The mRNA level of the AMPA receptor GluA1 was decreased, while the levels of GluA2 and GluA3 were increased in the model group. There was no significant difference in GluA4 mRNA levels between control and model groups. Resveratrol could up-regulate mRNA expression of GluA1 receptor but had no effects on the other studied receptors. Conclusion: These findings indicate that Resveratrol may improve cognitive function in $\mathrm{AD}$ by regulating synaptic transmission.

\section{P65}

A Review of the Prevalence of Musculoskeletal Disorders in Nurses Working in Public Hospitals in Tehran

\section{Mohammad Arabsorkhi Mishabi, Esmaeil Teymoori, Dorrin Nikbakht}

Department of Operation Room, Student Research Committee, Faculty of Paramedicine, Iran University of Medical Sciences, Tehran, Iran

The Neuroscience Journal of Shefaye Khatam, 2019; 7(3): P65

Introduction: Musculoskeletal disorders are one of the most important occupational health issues in the world today, especially in nursing, and can be a major contributor to stress that is associated with high costs and reduced productivity in this profession. The aim of this study was to determine the prevalence of musculoskeletal disorders in nurses. Materials and Methods: This research is a systematic review to achieve the desired goals from advanced search in valid databases such as Irandoc, Magiran, Sid, Ganj, Noor. A total of 50 related articles from 2011-2011 were found with the keywords (Musculoskeletal Disorders, Patient Displacement, Nurses), which is the result of this research. Results: $78.3 \%$ of nurses had symptoms of this disorder in one or more areas of their musculoskeletal disorders during the past 12 months. The average daily working hours for both men and women were $7 / 31 \pm 0 / 46$ hours. Based on these findings, there was a statistically significant relationship between gender, age, work history, occupation, BMI (body mass index), education level, occupational stress and exercise with organ disorders $(\mathrm{P}<0 / 05)$. Also, lumbar region disorders with the prevalence of $60.9 \%$ were the most common disorder among nurses. Conclusion: According to the results of this study, the prevalence of musculoskeletal disorders in nurses is high. In most articles, patient placement has the highest score. In order to reduce the disorder, it is suggested that the necessary training is provided to identify the risk factors of the factors and their adjustment modalities and regular exercises.
Effects of Cannabinoid Receptor Modulation on Voltage SAG in Cerebellar Purkinje Cells Under Whole Cell Patch Clamp Recording

\section{Zeinab Sadat Hosseini', Hasan Abbasian²}

${ }^{1}$ Student Research Committee, Mashhad Branch, Islamic Azad University, Mashhad, Iran

${ }^{2}$ Department of Neuroscience, Mashhad University of Medical Sciences, Mashhad, Iran

The Neuroscience Journal of Shefaye Khatam, 2019; 7(3): P66

Introduction: Cannabinoid receptors are part of the endocannabinoid system which are involved in different physiological procedures including memory, painsensation and appetite. In the Whole-cell patch-clamp recording, Voltage SAG is a short period decrease in RMS voltage which can be caused by quick change of loads like motor startup, or short circuit. This property can be affected by changes in channels modulated by specific receptors or ligands so can be one of comparison factors in pharmacological researchs. The purpose of this study was to investigate the effects of cannabinoid receptor modulators on voltage SAG in cerebellar purkinje cells under whole patch clamp recording. Materials and Methods: The voltage SAG in current clap mode here after IP injection of Harmaline, agonist WIN55, 2122 and antagonists AM251 and SR171614a (Rimonobant) then slice of cerebellar vermis prepared and under direct vision of purkinje cells and confirming the patch, recording done and in offline mode the sag ratios were caculated by Digidata 1440 and pclamp 10.1. The data were analysed by prism v6. Results: Voltage $\mathrm{SAG}$ in response to $-0.5 \mathrm{nA}$ stimulation charge in to the patch electrode showed significant increase in WIN group comparison to control and Harmaline groups. This voltage in response to- $0.4 \mathrm{nA}$ stimulation indicated significant increase in WIN and Rimonobant group. Also, this result gained at $-0.3 \mathrm{nA}$ stimulation. In -0.2 $\mathrm{nA}$ stimulation, voltage SAG had significant increase in WIN and AM251 groups. Voltage SAG in -0.1 nA had significant increase in all the groups. Conclusion: According to the results, the changes in the agonist group are higher than in others but antagonists showed improvement of that toward control group can be benefit effects on harmaline toxicity alongside of our previous behavioral study.

\section{P67}

Alteration of Frequency Related Patterns of Cerebellar Purkinje Cells Under Whole Cell Patch Clamp Recording from Harmaline-Induced Tremor Rats

Fateme Sadat Abolghasemi, Arash Khojaste ${ }^{\text {h }}$ Zonoozi', Benjamin Jason Whalley', Mohammad Shabani $^{3}$ Hassan Abbassian ${ }^{4}$ 
${ }^{1}$ Student Research Committee, Faculty of Medicine, Mashhad University of Medical Sciences, Mashhad, Iran

${ }^{2}$ Departement of Pharmacology, Faculty of Chemistry, Food and Nutritional Sciences and Pharmacy, University of Reading, Reading, UK

${ }^{3}$ Kerman Neuroscience Research Center, Neuropharmacology Institute, Kerman University of Medical Sciences, Kerman, Iran ${ }^{4}$ Department of Neuroscience, Faculty of Medicine, Mashhad University of Medical Sciences, Mashhad, Iran

The Neuroscience Journal of Shefaye Khatam, 2019; 7(3): P67

Introduction: Essential tremor (ET) is the most common neurological motor-related disorder leading to impairment in daily living activities. Despite great number of cannabinoid receptors in cerebellum and basal ganglia, areas mainly responsible for motor function, and the reports of tremor benefits among ET patients using cannabis, still not many research studies have been conducted in this issue. In this paper, the effects of WIN 55,212-2 (WIN) as an agonist and AM251 and Rimonobant as antagonists on tremor-induced rats were studied at the level of cellular recording. Materials and Methods: 4-week-old rats were randomly divided into 5 groups; 2 normal saline and harmaline-injected controls along with 3 WIN, AM251 and Rimonobanttreated groups. Cerebellar purkinje cells were under electrophysiological examinations using whole cell patch clamp recording and different aspects of their activities were evaluated; action potential frequency, peak amplitude, action potential half width, time to peak, after hyperpolarization (AHP) and instantaneous frequency were measured by digidata 1440 and pclamp10.1 software offline and analyzed by prim 6. Results: The results showed a significant increase in action potential frequency and instantaneous frequency in the group treated with WIN $(1 \mathrm{mg} / \mathrm{kg})$ compared to tremor-induced group. Peak amplitude was attenuated and AHP was decreased in WIN-treated rats. Further, time to peak and action potential half width were reduced by WIN, while AM251 did not show significant difference in these two above-mentioned factors comparing with harmalineinjected rats. Conclusion: Thus, application of WIN did not represent any association with normal control pattern while cannabinoids' antagonists tracked the standards to such an extent. Since cannabinoids effects are grabbing loads of attention in CNS-related motor discomforts and as concluded, cannabinoid receptor antagonists appeared to be more eligible in treating tremor-induced rats, they are considered as potential candidates for tremor management.

\section{P68}

The Neural Function of Cuneiform Nucleus in NonHypovolemic and Hypovolemic Hypotensive Rats: Single Unit Recording

Reza Mohebbati', Hassan Abbassian', Mohammad
Naser Shafei ${ }^{1,3}$, Sajad Sahab Negah', Ali Gorji ${ }^{2,4 *}$

'Department of Physiology, Faculty of Medicine, Mashhad University of Medical Sciences, Mashhad, Iran

${ }^{2}$ Department of Neurosciences, Faculty of Medicine, Mashhad University of Medical Sciences, Mashhad, Iran

${ }^{3}$ Neurogenic Inflammation Research Center, Mashhad University of Medical Sciences, Mashhad, Iran

${ }^{4}$ Institut für Physiologie, Universität Münster, Robert-KochStrasse, Münster, Germany

The Neuroscience Journal of Shefaye Khatam, 2019; 7(3): P68

Introduction: Hemorrhagic hypotension results from a life-threatening loss of blood and physiologically decreases baroreceptor discharge and leads to chemoreceptor stimulation, due to stagnant anoxia induced by decreased blood flow to the chemoreceptors. Cuneiform nucleus $(\mathrm{CnF})$ located in center of circuit that mediated autonomic responses related to stress. In this study, the $\mathrm{CnF}$ neurons activity in hypotension induced by hemorrhage and hydralazine, as an antihypertension drug, evaluated by blood pressure and single unit recording. Materials and Methods: Animals groups were: Control, Hemorrhage and Hydralazine. After anesthesia, femoral artery and tail vein of rats were cannulated for recording cardiovascular responses include in Systolic blood pressure (SBP), Mean arterial pressure (MAP) and heart rate (HR) and drug injection. Also, single unit recording performed from $\mathrm{CnF}$ neurons. Results: The results showed that in Hydralazine groups, maximal change $(\Delta)$ of SBP and MAP significantly decreased and HR significantly increased than Control group. Also, in Hemorrhage group, $\triangle \mathrm{SBP}$ and $\triangle \mathrm{MAP}$ significantly decreased and $\triangle \mathrm{HR}$ significantly increased than Control group. After the hypotension induction or hydralazine injection relatively significant increase in spike frequency as well as PSTH showed. Conclusion: Current study has been shown that $\mathrm{CnF}$ has a cellular function in the hypovovemic and non-hypovolemic hypotension in the rats. Probably mechanisms are relationship between $\mathrm{CnF}$ and other vasomotor nuclei and then existence of volume sensitive receptors that response to the hypovolemic hypotension.

\section{P69}

Reconstruction and Analysis of circRNA-miRNAmRNA Regulatory Network for Exploring Underlying Pathogenesis of Multiple Sclerosis (MS)

Mohsen Ahmadi', Reza Fotuhi Ardakani ${ }^{2}$, Seyed Amir Momeni $^{3}$, Mohammad Moghaddam ${ }^{3}$, Fatemeh Haji Esmaelei, Safoura Houshmand ${ }^{4}$, Soghra Nouri, Zahra Beiglou, Seyed Ali Kashfi

${ }^{1}$ Universal Scientific Education and Research Network (USERN) Office, Research and Development Division, Booali Medical Diagnostic Laboratory, Qom, Iran 
${ }^{2}$ Cellular and Molecular Research Center, Qom University of Medical Sciences, Qom, Iran

${ }^{3}$ Clinical Pathology Division, Booali Medical Diagnostic Laboratory, Qom, Iran

${ }^{4}$ Anatomical Pathology Division, Booali Medical Diagnostic Laboratory, Qom, Iran

${ }^{5}$ Department of Genetic, Qom Branch, Islamic Azad University, Qom, Iran

The Neuroscience Journal of Shefaye Khatam, 2019; 7(3): P69

Introduction: Multiple sclerosis (MS) is a multifactorial, inflammatory, and neurodegenerative disorder affecting the myelinated axons of the central nervous system causing neurological deterioration. Despite more than 40 years of MS research its etiology remains unknown. The current study was aimed at identifying key genes and molecular mechanism in MS. Materials and Methods: Gene expression datasets (GSE17846, GSE31568, GSE39643, GSE61741, GSE17048 and GSE21942) were downloaded from Gene Expression Omnibus (GEO) for integrated bioinformatics analysis. Interactions between differentially expressed miRNAs, mRNAs and lncRNAs were predicted and merged with the target genes. Co-expression of miRNAs, IncRNAs and mRNAs was selected to construct mRNAmiRNA-lncRNA interaction networks. Additionally, circRNA-miRNA-mRNA regulatory networks were visualized using Cytoscape. Gene Ontology (GO) and Kyoto Encyclopedia of Genes and Genomes (KEGG) pathway enrichment analyses were performed for the DEGs. Protein-protein interaction (PPI) networks were constructed, and transcription factors were annotated. Results: According to the cut-off criteria (adjusted P-value $<0.05$ and $|\log 2 \mathrm{FC}|>1.0$ ), a total of 457 miRNAs, 660 mRNAs and 23 lncRNAs were differentially expressed in MS. The DE mRNAs were mainly enriched in the nuclei and cytoplasm. Enrichment analysis for all the DE mRNAs showed that they mainly enriched in lymphocyte activation, Hematopoietic cell lineage and myeloid leukocyte activation. Subsequently, pathways interrelation analysis of hub genes was carried out using plug-in ClueGO v2.3.3. The miRNAs were linked to immunological and neurological pathways, and we exposed hsa-let-7b-5p, hsa-miR-664a-3p and hsa-miR-142-3p as promising blood-derived disease biomarkers in MS. Furthermore, we found that 3 miRNAs, 6 lncRNAs, 4 mRNAs and 3 TFs play important regulatory roles in the interaction network. Conclusion: This study revealed the potential pathways and circRNA, miRNA, mRNA, IncRNAs regulatory networks in MS which may contribute to a more comprehensive understanding of MS pathogenesis.

\section{P70}

The Effect of Eight Weeks of Water Exercises (Hydro Therapy) on Pain Relief and Muscle Spasm in

\section{Women with MS Disease in Bushehr Province}

\section{Parviz Farzadinia $^{1}$, Alireza Asadnia ${ }^{2}$, Aria Salehpour ${ }^{2}$}

${ }^{1}$ Department of Biology and Anatomical Sciences, Faculty of Medicine, Bushehr University of Medical Sciences, Bushehr, Iran ${ }^{2}$ Student Research Committee, Bushehr University of Medical Sciences, Bushehr, Iran

The Neuroscience Journal of Shefaye Khatam, 2019; 7(3): P70

Introduction: The present study evaluates the effect of 8 weeks of water therapy exercises on pain relief and muscle spasm in MS. exercising play a very important role in controlling these symptoms. Many researchers have tried to find ways to make exercising better. Research has shown that exercise and water exercises (hydro therapy) are one of the most effective ways to reduce pain and muscle spasm, especially in women with MS. Materials and Methods: This quasi-experimental study was performed on 45 women with lower extremity MS disease. In this research, the subjects were divided into three groups consisting 15 people. The control group did not perform any exercises. Second group performed non-water exercises (on parallel) and third group did special exercises in water for eight weeks, three days a week, and one hour per day, Parameters of pain and muscle spasm were studied on the first day, twentyeighth and fifty-sixth day, using descriptive statistics, one-way ANOVA, T-test, covariance analysis and paired t-test for moderate averages using SPSS18 software. Results: Statistical analyzes showed that patients who had regular water exercise for 8 weeks had a significant effect on pain and muscle spasm relief. They also tended to spend more time in water doing exercises without revealing pain or spasms. Conclusion: Sport activities and exercises play a very important role in controlling motor problems in patients with MS, especially water exercises, due to the possibility of reducing the damage and eliminating spasms and seeking pain relief. The results of other researchers are consistent with the results of this research.

\section{P71}

Expression Analysis of GAS8 and its Naturally Occurring Antisense RNA in MS Patients Versus Healthy Subjects

\section{Mona Patoughi, Soudeh Ghafouri-Fard}

Department of Biology, Science and Research Branch, Islamic Azad University, Tehran, Iran

The Neuroscience Journal of Shefaye Khatam, 2019; 7(3): P71

Introduction: Expressions of the Growth arrest specific 8 (GAS8) and its naturally occurring antisense RNA (GAS8-AS1) have been assessed in tumoral tissues of different origins. However, their association with immune-related disorders has been poorly 
understood. Materials and Methods: In the current study, we evaluated expression levels of these genes in 50 relapsing remitting multiple sclerosis (RRMS) patients compared with age- and sexmatched controls. Expression assays were performed on peripheral blood samples of study participants following extraction of total RNA and cDNA synthesis using blood, RNA extraction, and cDNA Reverse Transcription. TaqMan (Real Time PCR), The primers and probes sequences. Results: Expression of both genes were significantly higher in total MS patients compared with controls ( $\mathrm{P}=0.001$ and $\mathrm{P}<0.0001$ respectively). The difference in GAS8 expression was also significant in total female patients and females aged less than 50 when compared with the corresponding control subjects $(\mathrm{P}=0.002$ and 0.006 respectively). GAS8-AS1 was higher in male patients in both age-based subgroups compared with the corresponding healthy subjects $(\mathrm{P}<0.0001)$. Expressions of both genes were inversely correlated with age of male study participants but no other subgroups. GAS8-AS1 transcript levels had $99.6 \%$ accuracy in diagnosis of disease status in male subjects. Conclusion: The current study shows significance of GAS8 and GAS8-AS1 in the pathogenesis of MS and the putative role of GAS8AS1 as a diagnostic biomarker in a subset of patients.

\section{P72}

Effect of Oral Mucosal Mesenchymal Stem Cells on Brain Edema, Long-Term Neurologic and Motor Outcome and Anxiety in Experimental Traumatic Brain Injury

\section{Fateme Dehghanyan $^{1}$, Zahra Soltani ${ }^{2}$, Ali Farsinajad ${ }^{3}$}

${ }^{1}$ Department of Physiology and Pharmacology, Afzalipour Faculty of Medicine, Kerman University of Medical Sciences, Kerman, Iran

${ }^{2}$ Endocrinology and Metabolism Research Center, Institute of Basic and Clinical Physiology Sciences, Afzalipour Faculty of Medicine, Kerman University of Medical Sciences, Kerman, Iran

The Neuroscience Journal of Shefaye Khatam, 2019; 7(3): P72

Introduction: Traumatic brain injury (TBI) is a major public health problem in present society and annually causes disability and death in many people. Studies in recent decades have shown that mesenchymal stem cells (MSCs) could be have beneficial effects in neurodegenerative diseases such as TBI because of high capacity in proliferation and differentiation. In this study, the effect of oral mucosal MSCs treatment was evaluated on TBI- induced cerebral edema, long-term neurological and motor outcomes and anxiety. Materials and Methods: In this study, 84 Wistar adult male rats were used. Animals divided to four groups: sham, TBI, vehicle, stem cell (SC). Moderate diffuse traumatic brain injury was induced by marmarou's method. Oral mucosal MSCs $\left(2 \times 10^{6}\right.$ cells $)$ were injected intravenously
1 and $24 \mathrm{~h}$ after injury. The brain edema was determined by calculating brain water content $24 \mathrm{~h}$ and 21 days after the injury. Long-term neurological and motor outcomes were evaluated [(using neurologic severity score (NSS) and open field test (OFT), respectively] before injury, and 3, 7, 14 and 21 days post- injury. The anxiety was assessed using elevated plus maze (EPM) 3, 7, 14 and 21 days after the damage. Results: TBI was resulted in a significant increase in brain water content compared to sham group $(\mathrm{P}<0.001)$. The administration of MSCs reduced brain water content in $24 \mathrm{~h}$ and 21 days after injury $(\mathrm{P}<0.001)$. Lower NSS was observed in the group receiving $\mathrm{MSCs}$ in comparison with TBI group $(\mathrm{P}<0.001)$. The anxiety decreased in the group receiving MSCs compared to sham group $(\mathrm{P}<0.05)$. Conclusion: The results of this study show that administration of MSCs derived from the oral mucosa following TBI could probably reduce cerebral edema leading to the improvement of neurologic outcome and anxiety. Therefore the administration of MSCs can be suggested as a new and hopeful treatment in TBI patients.

\section{P73}

\section{Chrysin Impact on Brain Oxidative Damages} Induced by Immobilization in Rat

\section{Saeed Samarghandian 1, 2, Tahereh Farkhondeh, Sediqeh Jalali ${ }^{4}$, Fariborz Samini ${ }^{5}$}

${ }^{1}$ Noncommunicable Disease Research Center, Neyshabur University of Medical Sciences, Neyshabur, Iran

${ }^{2}$ Department of Basic Medical Sciences, Neyshabur University of Medical Sciences, Neyshabur, Iran

${ }^{3}$ Cardiovascular Diseases Research Center, Birjand University of Medical Sciences, Birjand, Iran

${ }^{4}$ Department of Biology, Payam Noor University, Tehran, Iran ${ }^{5}$ Department of Neurosurgery, Faculty of Medicine, Mashhad University of Medical Sciences, Mashhad, Iran

The Neuroscience Journal of Shefaye Khatam, 2019; 7(3): P73

Introduction: The aim of the current investigation is evaluation of the impact of chrysin $(\mathrm{CH})$ on brain oxidative damage induced following chronic stress in animal models. Materials and Methods: The rats were restrained for 1 hour daily for 3 weeks. During these weeks, all animals were daily injected with either vehicle or $\mathrm{CH}(10,20,30 \mu \mathrm{g} / \mathrm{kg})$. Results: In the stressed animals that received vehicle, the MDA level was significantly higher $(\mathrm{P}<0.01)$ and the levels of GSH and antioxidant enzymes were significantly lower than the non-stressed animals $(\mathrm{P}<0.05)$. $\mathrm{CH}$ also ameliorated the MDA and GSH content as well as antioxidant enzymes activities in stressed rats $(\mathrm{p}<0.05)$. Conclusion: The present study suggested that $\mathrm{CH}$ may be useful for the management of depressant-like effects induced by chronic stress via decreasing oxidative damage in the brain. 


\section{P74}

Co-Transplantation of Human Neurotrophic Factors Secreting Cells and Adipose-Derived Stem Cells in Rat Model of Multiple Sclerosis

\section{Shahnaz Razavi}

Department of Anatomical Sciences, Faculty of Medicine, Isfahan University of Medical Sciences, Isfahan, Iran

The Neuroscience Journal of Shefaye Khatam, 2019; 7(3): P74

Introduction: The presence of neurotrophic factors is critical for regeneration of neural lesions. Here, we transplanted combination of neurotrophic factor secreting cells (NTF-SCs) and human adipose derived stem cells (hADSCs) into a lysolecithin model of multiple sclerosis and determined the myelinization efficiency of these cells. Materials and Methods: In this experimental study, fifty adult rats were randomly divided into five groups: control, lysolecithin, vehicle, hADSCs transplantation and NTF-SCs/ hADSCs co-transplantation group. Focal demyelization was induced by lysolecithin injection into the spinal cord. In order to assess motor functions, all rats were scored weekly with a standard experimental autoimmune encephalomyelitis scoring scale before and after cell transplantation. Four weeks after cell transplantation, the extent of demyelination and remyelination, were examined with Luxol Fast Blue (LFB) staining. Also, immunofluorescence method was used for evaluation of oligodendrocyte differentiation markers including; myelin basic protein (MBP) and Olig2 in the lesion area. Results: Histological study show that somewhat remyelinzation in cell transplantation groups relative to others. In addition, the immunofluorescence results indicated that the MBP and Olig2 positive labeled cells were significantly higher in co-cell transplantation group than hADSCs group $(\mathrm{P}<0.05)$. Also, outcome of motor functional test show that significant improvement function in cell transplantation groups as compared to others $(\mathrm{p}<0.01)$. Conclusion: Our results indicated that the remyelinization process in co-cell transplantation group was better than other groups. Thus, NTF-SCs/ hADSCs transplantation can be proper candidate for cell based therapy in neurodegenerative diseases such as MS.

\section{P75}

Adjusting Effects of Sodium Valproate on Hippocampal Levels of NF-KB, S100B and GFAP Following the Alloxan Induced Diabetes

Behnam Amirpour-Najafabadi', Mahsa Gholami', 2, Parvin Zarie $^{1,2}$, Sirvan Hossieni', Mehdi Sadegh ${ }^{3}$

${ }^{1}$ Student Research Committee, Arak University of Medical Sciences, Arak, Iran
${ }^{2}$ Department of Biochemistry and Genetic, Faculty of Medicine, Arak University of Medical Sciences, Arak, Iran

${ }^{3}$ Department of Physiology, Faculty of Medicine, Arak University of Medical Sciences, Arak, Iran, Iran

The Neuroscience Journal of Shefaye Khatam, 2019; 7(3): P75

Introduction: Chronic diabetes with uncontrolled hyperglycemia causes neuropathy. Sodium valproate has neuroprotective and regenerative effect in neurodegenerative disease. We investigated the effectiveness of chronic valproate on diabetes induced modification in the hippocampal levels of NF-KB, AP-1, S100B and GFAP. Materials and Methods: 24 adult male C57B15/J mice were used. Diabetes was induced by alloxan $(150 \mathrm{mg} / \mathrm{kg}$; i.p.). Valproate were administrated (100 mg/kg; i.p) every 72 hours for two months. Fasting blood sugar were measured at the beginning and at the end of experimental procedure, then animals were killed, hippocampus were extracted and prepared for ELISA to assess biochemical factors. Results: Increased blood glucose due to alloxan induced diabetes were significantly reduced following the chronic valproate administration $(\mathrm{P}<0.05)$. Also, chronic valproate administration in diabetic animals were significantly reduced, elevated level of hippocampal NF$\mathrm{KB}, \mathrm{S} 100 \mathrm{~B}$ and GFAP $(\mathrm{P}<0.05)$.Conclusion: It seems valproate is able to adjust diabetes induced modification in biochemical factors of the hippocampus and might be effective in diabetic neuropathy.

\section{P76}

\section{Occupational Therapy in Multiple Sclerosis}

Bahareh Zeynalzadeh Ghoochani, Seyed Alireza Derakhshanrad

Department of Occupational Therapy, Faculty of Rehabilitation Sciences, Shiraz University of Medical Sciences, Shiraz, Iran

The Neuroscience Journal of Shefaye Khatam, 2019; 7(3): P76

Introduction: Multiple sclerosis is known as one of central nervous system diseases that is caused by lesions in myelination leading to different neurologic signs and symptoms. Due to various problems of these patients choosing proper intervention should be highlighted in national health agenda and treatment should be applied as soon as possible. Occupational therapy is one of major therapeutic options concerning individual's Health status. Materials and Methods: Although drugs can hinder the progress of disease in these patients, many patients would still experience restrictions. Rehabilitation can provide services to maximize performance, prevention and enable individuals. Decreasing disabilities is the primary goal of rehabilitation. Rehabilitation team members can educate patients and intervene to promoting health as soon as the disorder is diagnosed even if the disabilities are not still obvious and clinically 
observed. So in this study we are going to define some professional assessment and intervention in this disorder. Results: In order to evaluate these patients indexes such as Performance Assessment of Self-care Skills, Barthel, FIM, Kurtzke Scale would really help. Additionaly Multiple Sclerosis Quality of life Inventory, Pain Effect Scale, Fatigue Assessment Battery for Multiple Sclerosis, Fatigue Impact Scale, Dynamic Gait Index (DGI-P), Impact of Visual Impairment Scale, Perceived Deficits Questionnaire, Balance Evaluation Systems Test, Berg test, Mental Health Inventory, Wisconsin Card Sorting Test and Modified Social Support Survey would report patient's condition to design and plan intervention program. Conclusion: According to various assessments, patient's problems and the severity of involvements interventions would be provided. The most important therapy field would be related to fatigue, weakness, spasticity, balance and coordination, sensory problems, transfer and mobility cognitive problems, activity of daily living, vocational rehabilitation and psychosocial. In the light of the above, occupational therapists can benefit from these assessments and treatment fields to enrich their early intervention in these fields.

\section{P77}

The Effect of Bacillus Coagulans on NLRP1 Gene Expression and the Improvement of the Inflammasome Pathway in the Cuprizone-Induced Mouse Model of Demyelination

\section{Saba Sadeghi Rashed ${ }^{1}$, Saba Taheri', Javad Arasteh ${ }^{1}$}

${ }^{1}$ Department of Biology, Central Tehran Branch, Islamic Azad University, Tehran, Iran

${ }^{2}$ Department of Biology, Islamshahr Branch, Islamic Azad University, Islamshahr, Iran

The Neuroscience Journal of Shefaye Khatam, 2019; 7(3): P77

Introduction: Multiple sclerosis is an autoimmune disease of the central nervous system correlated with inconvenient activation of lymphocytes, hyperinflammatory responses, demyelination, and neuronal loss. Inflammasomes are cytosolic multiprotein complexes responsible for the activation of inflammatory responses and they initiate innate immunity by recruitment and activation of the pro-inflammatory Caspase-1. The activated form of caspase- 1 provides, in turn, activation of IL-1 $\beta$ and IL-18. NLRP1 was the first discovered inflammasome. It is expressed by neurons, microglia, and astrocytes and was shown to play a major role in neuronal death and CNS inflammation. This study was designed to reveal the effect of Bacillus-coagulans T4 IBRC-M10791, a novel and domestic strain isolated from Tarhana on the expression of NLRP1 in the cuprizone mouse model. Materials and Methods: Adult female $\mathrm{C} 57 \mathrm{bl} / 6$ mice were divided into 5 groups
(7 mice in each group). group 1: normal control, group 2: cuprizone control (oral administration of cuprizone $0.2 \% \mathrm{w} / \mathrm{w}$ for $5 \mathrm{weeks}$ ), group 3: probiotic control (oral administration of Bacillus coagulans at a concentration of $1 \times 10^{9} \mathrm{cfu} / \mathrm{ml}$ for 4 weeks), group 4: prevention group (oral administration of Bacillus coagulans for 2 weeks then cuprizone for 5 weeks) and group 5: Improvement group (oral administration of cuprizone for 5 weeks then Bacillus coagulans for 4 weeks). To confirm and ensure the formation of demyelination in the corpus callosum, the luxol fast blue staining was used and then the expression of the NLRP1 gene in peripheral blood of different groups was evaluated by real-time PCR. Results: The results of this experiment indicated that there was a significant difference between both prevention and Improvement groups with cuprizone control $(p<0.05)$. Conclusion: We investigated that the daily intake of Bacillus coagulans could be improving in the prevention and improvement of MS.

\section{P78}

$\begin{array}{ll}\text { Risperidone Accelerates } & \begin{array}{l}\text { Bone Loss in Mice with } \\ \text { Autistic-Like Deficits }\end{array} \\ \text { Lipopolysaccharides } & \begin{array}{l}\text { Exposure } \\ \text { Expos by Maternal }\end{array}\end{array}$

Zahra Namvarpour', Abdollah Amini', Mozhdeh Namvarpour ${ }^{2}$

${ }^{1}$ Institute for Cognitive Science Studies (ICSS), Tehran, Iran

${ }^{2}$ Department of Biology and Anatomical Sciences, Faculty of Medicine, Shahid Beheshti University of Medical Sciences, Tehran, Iran

The Neuroscience Journal of Shefaye Khatam, 2019; 7(3): P78

Introduction: Patients with neurodevelopmental disorders, usually suffer from low bone density. Many studies have revealed a higher risk of fracture after treatment with the atypical antipsychotic drug Risperidone (RIS), which is used to treat such disorders. It remains debatable whether neurodevelopmental disorders are considered by low bone mass or pharmacotherapy may be the reason. This study evaluated the effects of risperidone on different aspects of bones in mice with lipopolysaccharide (LPS)-induced autistic-like deficits. Materials and Methods: Five groups of male mice were examined: LPS+Saline, which were exposed to LPS prenatally and received saline on days 28-56 postnatal; Saline +RIS, which received RIS orally in the same days; LPS+RIS, which were exposed to LPS prenatally then received RIS; Saline + Saline and Saline + RIS Vehicle, which were included as control groups. On the $56^{\text {th }}$ day postnatal, animals were assessed for autistic-like behaviors. Then their bones were taken for biomechanical, immunohistological, stereological, and molecular evaluations. Results: Maternal LPS exposure resulted in deficits in all behavioral tests and RIS ameliorated these behaviors 
$(p<0.01) \&(p<0.05)$. The administration of LPS and RIS individually caused reduction in the biomechanical factors of bone. The numerical density of osteocalcinpositive cells was significantly decreased in the mice in these groups. These mice also had decreased RUNX2 and osteocalcin gene expression. When LPS mice were treated with Risperidone, these conditions were $\operatorname{accelerated}(p=0.000)$. Conclusion: In the LPS mice model of neurodevelopmental disorders, bone stability and mass were reduced. This condition worsened when these animals were treated with Risperidone.

\section{P79}

Implementing A Dynamic Fuzzy State Estimator for Predicting M1-M2 Phenotypes Switch in Post Ischemic Stroke

\section{Ashkan Sedigh ${ }^{1}$, Amir Reza Kachooei, Shima Haghbin ${ }^{2}$}

${ }^{1}$ Faculty of Medicine, Mashhad University of Medical Sciences, Mashhad, Iran

${ }^{2}$ Department of Mechanical Engineering, Faculty of Engineering, Birjand University, Birjand, Iran

The Neuroscience Journal of Shefaye Khatam, 2019; 7(3): P79

Introduction: The role of Microglia cells after ischemic stroke has been approved in the neuroinflammation procedure. However, the mechanism of switching activated microglia to M1-M2 phenotypes is still unknown to the scientists. This switching process contains an uncertain dynamic and non-linear system that most computational methods including probabilistic reasoning could be very difficult to handle model estimation and further in-silico analysis. Materials and Methods: A dynamic fuzzy state estimator has been designed to estimate the M1-M2 states. Input data for training our model is the M1 microglia, which is activated by lipopolysaccharide produces proinflammatory cytokine and M2 phenotypes, which is activated by interleukin (IL-4) and expresses antiinflammatory mediators in the different stages. Fuzzy rules and inference engine are designed to setup our dynamic system within a k-fold training and testing sets. Results: In order to achieve a better understanding of the M1-M2 switching process after microglia activation, computational model within dynamic state estimators (finite states) is considered. In our implemented method, Fuzzy systems, is simulated with Gaussian membership functions and Mamdani Interpretation. The accuracy of the output model is also related to the input data which guarantees the computation robustness in estimation. Therefore, Predicting M1 and M2 microglia switch procedure could assist researchers to prevent Neurodegenerative Diseases. Conclusion: Our research demonstrates a computational model based on the fuzzy dynamic estimator. Early estimation mechanism may aid researchers to prevent neurodegenerative disease due to the neuroinflammation after ischemic stroke.

\section{P80}

Silymarin-Loaded Chitosan Nanoparticles Attenuate Anxiety-Like Behaviors in A Mice Model of Schizophrenia Induced by Ketamine

Tahmineh Sharifian ${ }^{1}$, Akbar Hajizadeh Moghaddam ${ }^{1}$, Vahid Hasantabar ${ }^{2}$

${ }^{1}$ Department of Biology, Faculty of Basic Sciences, University of Mazandaran, Babolsar, Iran

${ }^{2}$ Department of Organic-Polymer Chemistry, Faculty of Chemistry, University of Mazandaran, Babolsar, Iran

The Neuroscience Journal of Shefaye Khatam, 2019; 7(3): P80

Introduction: Schizophrenia is a neuropsychiatric disorder that is characterised by psychotic symptoms like positive, negative, and disorganised. Silymarin is a polyphenolic flavonoid with strong antioxidant and antiinflammatory activities, but clinical application limited due to its poor aqueous solubility and low bioavailability. The purpose of this study was to evaluate the silymarinloaded chitosan nanoparticles (SM-CH-NPs) effects on anxiogenic-like behaviors and hyperlocomotion in a mice model of schizophrenia. Materials and Methods: Animals were divided into five treatment groups $(n=7)$. Animals received distilled water, Aripiprazole 5, SM or SM-CH-NPs (20 mg/kg/day) orally for 30 days, and from the 15 th to the 30 th day, they were injected with ketamine $(20 \mathrm{mg} / \mathrm{kg} /$ day) intraperitoneally. Anxiety-like behaviors and locomotor activity were assessed by open field tests. Results: Our results showed that ketamineinduced anxiogenic-like behaviors and hyperactivity. Treatment with SM-CH-NPs showed a significant increase in center duration and decrease in locomotor activity compared to the ketamine-treated group. Conclusion: Our results show that the administration of SM and SM-CH-NPs attenuate anxiety-like behaviors in a mice model of schizophrenia induced by ketamine

\section{P81}

Everolimus Attenuates Glutamate-Induced PC12 Cells Death

\section{Mohaddeseh Sadat Alavi', , Sahar Fanoudi, , Hamid Reza Sadeghnia ${ }^{1,3}$}

${ }^{1}$ Pharmacological Research Center of Medicinal Plants, Mashhad University of Medical Sciences, Mashhad, Iran

${ }^{2}$ Division of Neurocognitive Sciences, Psychiatry and Behavioral Sciences Research Center, Mashhad University of Medical Sciences, Mashhad, Iran

${ }^{3}$ Department of Pharmacology, Faculty of Medicine, Mashhad University of Medical Sciences, Mashhad, Iran

The Neuroscience Journal of Shefaye Khatam, 2019; 7(3): P81 
Introduction: Glutamate-induced neuronal cell death plays a key role in neurodegenerative disorders such as Alzheimer's disease. Some recent studies reported the potential immunomodulatory and neuroprotective properties of inhibitors of serine-threonine kinase, mTOR (mammalian target of rapamycin). However, no study was conducted about the neuroprotective potential of everolimus (EVR), a selective and potent mTOR inhibitor. Therefore, the present study was designed to investigate whether EVR has protective effects against glutamate-induced neuronal injury and elucidate the underlying molecular mechanisms. Materials and Methods: PC12 cells were concurrently treated with EVR (0-40 $\mathrm{nM})$ and glutamate $(8 \mathrm{mM})$ for $24 \mathrm{~h}$. Then, the cells viability, apoptosis rate, and proteins involved in apoptosis (caspase-3, bax and bcl-2) were measured using MTT, annexin V-PI and immunoblotting assays. Results: Analyzing the protective effect of different concentrations of EVR (0-40 nM) against glutamateinduced cytotoxicity revealed a significant increase in cell viability in co-treatment regimen $(p<0.01)$. Also, $\operatorname{EVR}(40 \mathrm{nM})$ significantly $(\mathrm{p}<0.01)$ inhibited glutamateinduced apoptosis through depressing the elevation of bax/bcl-2 ratio and cleaved caspase-3 expression, in a concentration-dependent manner. Conclusion: The results demonstrated, for the first time, that EVR could protect against glutamate-mediated PC12 cell death via inhibiting apoptosis.

\section{P82}

Investigating the Severity of Fatigue and its Relationship with Circadian Rhythm Sleep Disorders in Patients with Multiple Sclerosis in Khorramabad at 2017

\section{Nasrin Galehdar', Mohammad-Hasan Imani-Nasab ${ }^{2}$}

${ }^{1}$ Faculty of Paramedical, Lorestan University of Medical Sciences, Khorramabad, Iran

${ }^{2}$ Department of Public Health, Faculty of Health and Nutrition, Lorestan University of Medical Sciences, Khorramabad, Iran

The Neuroscience Journal of Shefaye Khatam, 2019; 7(3): P82

Introduction: multiple sclerosis is one of the most common chronic autoimmune diseases in young people and women in various societies. Fatigue and sleep disorders are the most common symptoms associated with multiple sclerosis. The aim of this study was to determine the severity of fatigue and its relationship with circadian rhythm sleep disorders in patients with multiple sclerosis in khorramabad city. Materials and Methods: this was a descriptive cross-sectional study. The statistical population of the study was all the patients with multiple sclerosis referred to the MS association of Khorramabad. The sampling method was a simple random sampling from the list of patients with MS registered in the MS association. The data collection tools were two questionnaires of PSQI and FSS. The data were analyzed by the SPSS 18 software. Descriptive and analytical statistics were used to measure the associations. Finally, $\mathrm{P}<0.05$ was considered as statistically significant. Results: In this study, out of 64 patients, 18 (28.1\%) were men. The mean score of circadian rhythm sleep disorders was different significantly on the basis of the type of insurance. There was no significant correlation between age and the mean score of circadian rhythm sleep disorders. The mean score of fatigue severity was significantly correlated with gender, but there was no significant relationship with consumption of hypnotics and type of insurance. The correlation between the total score of circadian rhythm sleep disorders and fatigue severity was statistically significant. Interventions to reduce fatigue severity and sleep disorders are needed to improve the health of these patients. Conclusion: It is suggested that in future studies, this type of study should be done with a larger sample population and in different populations.

\section{P83}

Evaluation of The Effect of Crocin on Behavioral, Motor and Cognitive Functions in Autistic Model Rats

Fatemeh Rezamohammadi, Mehrnoush Rahmani, Abbas Ali Vafaei, Abbas Ali Taherian, Ali Rahidypour

Student Research Committee, Department of Physiology, Faculty of Medicine, Semnan University of Medical Sciences, Semnan, Iran

The Neuroscience Journal of Shefaye Khatam, 2019; 7(3): P83

Introduction: Previous studies have shown that autism, or self-preservation, is a type of neurodegenerative disorder commonly seen in social communication and relations. Previous studies suggested that crocin, as the main combination of saffron, had potent antioxidant effects and probably had a beneficial effect on autismrelated disorders. Accordingly, the aim of this study was to determine the effect of crocin on behavioral, motor and cognitive functions in autistic model rats in experimental rats. Materials and Methods: In this experimental study, valproic acid was injected on day 12 of pregnancy (when the embryonic development of the neural tube occurs) for induction of autism. Also, some groups were injected with crocin (15 And $30 \mathrm{mg} / \mathrm{kg}$ body weight) or vehicle were placed intraperitoneally. On the other hand, all mice were tested on days 30 and 60 (Maze Plus, Hot plate, Rotarod, Open field, Morris Water Maze). Results: Data analysis showed that injection of valproic acid can cause behavioral disorders including decreased social interactions, increased repetitive behaviors, increased pain tolerance thresholds, increased anxiety, decreased behavioral behaviors, reduced balance power and motor learning, 
and cognitive change in rat infants undergo postnatal evaluation, which is usually done on days 30 and 60 , and the administration of crocin can significantly modify these abnormalities. $(\mathrm{P}<0.05) \quad$.Conclusion: These findings showed that crocin can modify behavioral, motor and cognitive functions in autistic model mice and may be effective in the pharmacological treatment of autistic dysfunction.

\section{P84}

Comparing Effects of Nicorandil and Dimethyl Fumarate in a Rat Model of Cerebral Ischemia

Maryam Owjfard I, 4, Mohammad Reza Bigdeli, 2, Anahid Safari', Mohammad Reza Namavar ${ }^{4,5}$

${ }^{1}$ Department of Animal Sciences and Biotechnology, Faculty of Life Sciences and Biotechnology, Shahid Beheshti University, Tehran, Iran

${ }^{2}$ Institute for Cognitive and Brain Science, Shahid Beheshti University, Tehran, Iran

${ }^{3}$ Stem Cells Technology Research Center, Shiraz University of Medical Sciences, Shiraz, Iran

${ }^{4}$ Clinic Neurology Research Center, Shiraz University of Medical Sciences, Shiraz, Iran

${ }^{5}$ Histomorphometry and Stereology Research Center, Shiraz University of Medical Sciences, Shiraz, Iran

The Neuroscience Journal of Shefaye Khatam, 2019; 7(3): P84

Introduction: Blood-brain barrier (BBB) disruption and production of oxygen free radicals are major pathogenic mechanisms in ischemic stroke. It is suggested that dimethyl fumarate (DMF) may improve BBB integrity and neurological function in stroke and its effects might be mediated via activation of nuclear factor erythroid 2-related factor 2 (Nrf2) pathway. Nicorandil protects the brain against ischemic injury through its antiinflammation and anti-apoptotic properties. Current research attempted to elucidate the effects of DMF and nicorandil on BBB integrity and neurological function recovery of rats after middle cerebral artery occlusion (MCAO) and clarify the NF- $\mathrm{KB}$ and Nrf2-dependent mechanisms modulated by these drugs. Materials and Methods: Rats were subjected to $1 \mathrm{~h} \mathrm{MCAO, \text {followed }}$ by 3 days of reperfusion. 48 Sprague-Dawley male rats were allocated into four groups $(\mathrm{n}=12)$ : the sham group was just subjected to surgery stress; vehicle, DMF, and nicorandil groups, after MCAO received vehicle, 30 $\mathrm{mg} / \mathrm{kg}$ DMF, and $5 \mathrm{mg} / \mathrm{kg}$ nicorandil, respectively for 3 days after MCAO. Modified neurological severity scores was evaluated every day. Triphenyltetrazolium chloride was just utilized to approve MCAO and BBB disruption was evaluated by extravasation of Evans blue. RT-PCR was done to analyze the expression levels of nuclear factor (NF)- $\mathrm{kB}$ and Nrf2. Results: Both drugs improved ischemia-induced BBB disruption and promote the expression of Nrf2, however, just DMF significantly improved neurobehavioral function and the expression of NF- $\mathrm{kB}$ was only promoted by nicorandil. Conclusion: DMF improved neurobehavioral outcome and BBB disruption in ischemic stroke, probably through upregulation of Nrf2 but nicorandil did not have prominent effect on stroke.

\section{P85}

Evaluation of Selenium Supplementation in Ischemic Stroke Outcome

\section{Athena Sharifi Razavi, Hamed Jafarpour}

Department of Neurology, Faculty of Medicine, Mazandaran University, Sari, Iran

The Neuroscience Journal of Shefaye Khatam, 2019; 7(3): P85

Introduction: Selenium is a trace element essential to human health, that protect against cellular damage by oxygen radicals through selenoproteins. Ischemic stroke is associated with the generation of oxygen free radicals resulting in a condition of oxidative stress. Supplementing stroke patients with antioxidant nutrients may improve survival. Materials and Methods: This was a randomized, double-blind ,placebo controlled study on consecutive ischemic stroke patients admitted in Buali sina Hospital, Sari, Iran, during 2015-2017. Inclusion criteria was accepted ischemic stroke by brain CT or MRI during last 72 with volume of at list one third of MCA territory. The patients were randomized into two therapeutic groups, one receiving Vial selenium 2000 microgram stat and 1000 microgram daily for 5 days and the other $40 \mathrm{cc}$ normal saline stat and $20 \mathrm{cc}$ daily for 5 days. Primary outcome measures with National Institutes of Health Stroke Scale(NIHSS) and modified Rankin Scale (MRS). Secondary Outcome Measures with Barthel index 3 months after discharge. Results: 40 ischemic stroke patients (18 females, 22 males) with mean age of $68.2 \pm 10$ years were investigated. Differences in NIHSS and Barthel index were not significant between the two groups; but differences in $\mathrm{mRS}$ was significant in case group $(p<0.00)$. Conclusion: Selenium can improve short term ischemic stroke outcome, but it not influences the long term outcome.

\section{P86}

Compare the Effect of Involuntary Exercise to Anxiety in Male and Female Rats with PTSD (As a Neurodegenerative Disease)

Razie Mirjalili, Elahe Shokouh ${ }^{I}$, Niloufar Sayah Dehkordi $^{1}$, Reza Afsari ${ }^{1}$, Sakineh Shafia ${ }^{2}$

${ }^{1}$ Student Research Committee, Faculty of Medicine, Mazandaran University of Medical Sciences, Sari, Iran

${ }^{2}$ Department of Physiology, Faculty of Medicine, Mazandaran University of Medical Sciences, Sari, Iran 
The Neuroscience Journal of Shefaye Khatam, 2019; 7(3): P86

Introduction: Post-traumatic stress disorder (PTSD) is a complex syndrome resulting from the exposure to a life-threatening event. These mental illnesses have detrimental effects on anxiety, cognitive abilities, and extinction. The risk of psychosis in women is twice that of men. The severity and durability of symptoms are also higher in women. There are differences in emotional related neuronal circuits between two sexes. Symptoms of anxiety disorders in women in different phase's reproductive life (puberty, postpartum and menopause) exacerbated. In this study we investigated difference in response to involuntary exercise in treatment of anxiety in male and female PTSD rats. Materials and Methods: We used to single prolonged stress (SPS) model as animal model for PTSD. The rats divided two groups; SPS and Sham groups. Rats in SPS group were exposed to (restraint for $2 \mathrm{~h}$, forced swimming for $20 \mathrm{~min}$ and ether anesthesia) and were then kept undisturbed for 14 days. After that, all of animals were subjected to moderate treadmill running (4 weeks, 5 days per week) followed by EPM task for anxiety Survey. Results: SPS male and female rats exhibited increased anxiety levels in the elevated plus maze but anxiety levels in female rats were more than male rats. Moderate treadmill exercise alleviated the SPS-induced alterations in terms of anxiety levels. Female SPS rats in exercise group had better responses in EPM test than male group. Female rats had higher resilience to treatment in anxiety test. Conclusion: Probability is given better response in female rats to exercise is as a result of the impact of exercise on the serotonergic system and interaction between serotonergic system and gonadotropic system.

P87

Anti-Proliferative Effect of Nano-Niosomal Formulation of Curcumin Against Glioblastoma Stem/Like Cells

Mohammad Jalili-Nik', Fatemeh Ariakia ${ }^{2}$, Amir Reza Afshari', Sajjad Sahab Negah ${ }^{2}$

${ }^{1}$ Department of Medical Biochemistry, Faculty of Medicine, Mashhad University of Medical Sciences, Mashhad, Iran

${ }^{2}$ Department of Neuroscience, Faculty of Medicine, Mashhad University of Medical Sciences, Mashhad, Iran

${ }^{3}$ Department of Pharmacology, Faculty of Medicine, Mashhad University of Medical Sciences, Mashhad, Iran

The Neuroscience Journal of Shefaye Khatam, 2019; 7(3): P87

Introduction: The glioblastoma cancer stem cells (GSCs) can contribute to tumor initiation, recurrence and also resistance to current treatments. In the present study, curcumin was encapsulated in a noisome nanoparticle, termed niosomal-curcumin (N-CM). The aim of this study was to evaluate the anti-cancer effects of N-CM on GSCs in comparison with free curcumin (CM). Materials and Methods: The cancer stem cells were isolated from the glioblastoma tumor tissue of a 50-year-old man. To evaluate cytotoxicity effects of $\mathrm{N}-\mathrm{CM}$, proliferation, viability, cell cycle, apoptosis, and ROS generation were assessed on GSCs. Results: $\mathrm{N}-\mathrm{CM}$ significantly decreased cell viability in a dosedependently manner. N-CM treatment leads to higher accumulation of cells in the SubG1 phase (apoptotic) in comparison with $\mathrm{CM}$. N-CM trigger apoptosis through the increasing Bax while decreasing $\mathrm{Bcl} 2$ expression. We also indicated an elevated amount of oxygen free radicals, and a declined expression of NF- $\mathrm{BB}$ and IL-6, which may be explained cytotoxicity of $\mathrm{N}-\mathrm{CM}$ against GSCs. Conclusion: These results introduce N-CM as a potent anti-tumor agent.

\section{P88}

Enhanced Neural Differentioation of Epidermal Neural Crest Stem Cell by Synergistic Effect of Lithium Carbonate and Crocin on BDNF and GDNF Expression as Neurotrophic Factors

\section{Shirin Ahmadi, Mohammad Nabiuni, Mohammad Tahmaseb, Elaheh Amini}

Department of Cellular and Molecular Biology, Faculty of Biological Sciences, Kharazmi University, Tehran, Iran

The Neuroscience Journal of Shefaye Khatam, 2019; 7(3): P88

Introduction: Neurodegenerative diseases are incurable and debilitating conditions that result in progressive degeneration of nerve cells. Due to the complexity of conditions in neurodegenerative diseases, combination therapy including cell therapy and drug therapy is important as a new therapeutic strategy. Epidermal neural crest stem cells (EPI-NCSCs) are as one of the best option in cell therapy for various neurological diseases. In this study, the effect of Lithium carbonate and Crocin, with considering of their effects on cellular signaling pathways and neuroprotective properties were investigated on expression of neurotrophic factors BDNF and GDNF in EPI-NCSCs. Materials and Methods: EPI-NCSCs were isolated from the Whisker hair follicles of the 2-week-old male Wistar rat and cultured in collagen-coated culture plates $(1 \mathrm{mg} / \mathrm{ml})$. After EPINCSCs emigration, adhering cells removed by trypsin and subsequently subcultured. Then, after 72 hours of treatment with different concentration of drugs [Lithium $(0.1,0.5,1,1.2,1.4,1.6,1.8,2,4,8 \mathrm{mM})$, Crocin (12.5, $50,100,200,500,1000,1500,2000,2500 \mu \mathrm{M})$ and lithium $(1 \mathrm{mM})+$ Crocin $(12.5,50,100,500,1000,1500$, $2000,2500 \mu \mathrm{M})]$, and trial doses were selected by MTT assay. The cells were treated with selected concentration (Lithium $1 \mathrm{mM}$, Crocin $1.5 \mathrm{mM}$, Lithium $1 \mathrm{mM}$ and Crocin $1 \mathrm{mM}$ ) for 7 days. Results: The Real Time PCR results indicated an increasing in expression of $\mathrm{BDNF}$ 
and GDNF in treated cells as compared with control $(* \mathrm{p}<0.05, * * \mathrm{p}<0.01$ and $* * * \mathrm{p}<0.001)$. Conclusion: The results in this study confirmed and supported the neuroprotective/neurogenesis effects of Lithium and Crocin, it also showed that the proposed protocol can be used to increase EPI-NCSCs differentiation potential into neural cells in cell therapy and combination therapy of neurodegenerative diseases.

\section{P89}

Evaluating the NARX and ELMAN for the Modeling of Epileptic EEG Signal

Seyed Soroosh Pedram, Seyed Javad Hashemi', Mohammad Ravari', Saleh Lashkari ${ }^{2}$

${ }^{1}$ Department of Biomedical Engineering, Mashhad Branch, Islamic Azad University, Mashhad, Iran

${ }^{2}$ Department of Biomedical Engineering, Imam Reza International University, Mashhad, Iran

The Neuroscience Journal of Shefaye Khatam, 2019; 7(3): P89

Introduction: The epilepsy can cause anaesthesia or seizures in peoples with epilepsy because of the Creating of heterogeneity in the pattern of neuronal activity that it is different in terms of being local or general. In the procedure of cure and choosing an effective way to control and betterment of the sickness, the EEG modeling can have a crucial role. Using the neural networks is one of the greatest Computational intelligence tools for the modeling of chaotic and non-linear signals which is used as the main target of this article. Materials and Methods: The purpose of this article is to evaluate two recurrent ELMAN and NARX neural networks for modeling the EEG signal. These two neural networks have been evaluated using complex modeling and predictive time series estimation. The data used in this research were obtained from the surface electrodes, Deep electrodes and strip electrodes in normal state, before seizure and during an epileptic seizure which categorized in to two healthy and epileptic groups. White noise is used as input for the modeling of EEG signal using NARX and in the ELMAN modeling, previous inputs are applied as input for the neural network. The benchmark for this project was MSE and Fitness. Results: The modeling of the EEG signal using ELMAN neural network had a precision of $82 \%$ and a sensitivity of $83 \%$. On the other hand, the NARX neural network resulted a $96 \%$ precision and $95 \%$ sensitivity. The epoch number is a parameter for evaluating the processing speed in neural network which were greater in NARX neural network. Conclusion: The NARX neural network achieved a higher precision and lower errors which concludes a better performance rather than ELMAN neural network in modeling the Epileptic EEG signal. Also, this neural network can be used in chaotic systems as a controller. This effective modeling that is done by NARX neural network can help sick people in predicting and controlling the seizures and also in determining the dose of their medicine.

\section{P90}

Zinc Oxide Nanoparticles Synthesized by the Green Method from the Extract of the Hyssopus Officinalis Plant and Their Anti-Cancer Effect on Brain Cells and Effect on the Toxicity of These Nanoparticles on Brain Tissue: In Vivo and in Vitro Studies

Ghasem Rahimi', 2, Masoud Homayouni Tabrizi ${ }^{1,2}$

${ }^{1}$ Department of Biology, Mashhad Branch, Islamic Azad University, Mashhad, Iran

${ }^{2}$ Department of Chemistry, Mashhad Branch, Islamic Azad University, Mashhad, Iran

The Neuroscience Journal of Shefaye Khatam, 2019; 7(3): P90

Introduction: The explicit bio-safety issue and potential hazard of nanoparticles (NPs) on various organelles can be considered as a major challenge. Hence, there is a need to appraise the safety problems of these NPs. In the present study, we have determined the green synthesis of $\mathrm{ZnO}$ nanoparticles using Hyssop (Hyssopus officinalis) extract and their effects on U87 cell line and BALB/c mice. Materials and Methods: The cytotoxicity of the ZnO NPs was assessed on U87 cell line by MTT test after characterization with various analytical techniques. The weight of brain tissue and histopathological assessments carried out as in vivo analysis in BALB/c adult male mice. Results: The obtained IC50 for U87 treated with ZnO NPs for 24 and 48 hours Respectively was $35 \mu \mathrm{g} / \mathrm{ml}$ and $23 \mu \mathrm{g} /$ $\mathrm{ml}$. From the aspect of histopathological, they did not change, but these nanoparticles slightly increased the weight of the brain tissue. All expenses of this research were done personally. Conclusion: The results of the analysis of MTT assay for u87 cell lines showed significant IC50 values, with increasing concentrations and increasing time, had a greater fecundity effect on the cells. The results obtained in this work contribute to increase the knowledge on the genotoxic and cytotoxic potential of $\mathrm{ZnO}$ NPs in general, and specifically on human neuronal cells, but further investigations are required to understand the action mechanism underlying the cytotoxic and genotoxic effects observed.

\section{P91}

Therapeutic Effects of Rosa Damascena as an Ancient Herb on Neuro- Inflammatory Disorders

\section{Zahra Baradaran, Farimah Beheshti}

Neuroscience Research Center, Torbat Heydariyeh University of Medical Sciences, Torbat Heydariyeh, Iran

The Neuroscience Journal of Shefaye Khatam, 2019; 7(3): P91 
Introduction: Rosa damascena ( $\mathrm{R}$. damascena) is an erect shrub from the Rosaceae family. Flowers of this plant are large, showy and colorful. R. damascena today are highly cultivated all over the world, including Iran (especialy in Kashan). In addition to its perfuming effect, flowers, petals and hips (seed-pot) of R. damascena are used for medical purposes. Several pharmacological properties including antibacterial, antioxidant, antiinflammatory, hypnotic, antidiabetic, antidepressant and relaxant effects have been reported for this plant. Materials and Methods: We searched the literature available in Scopus, PubMed, Google scholar and Science Direct databases for English articles published until 2000. For this purpose, we used 3 keywords for R. damascena, 15 for animal models and 10 for nervous system disorders. Results: This review article summarized a variety of in vitro and in vivo studies in order to find out the effects of $\mathrm{R}$. damascena in nervous system. It was shown in the articles methanolic extract of R. damascena improves spatial learning and memory parameters. The increase of the dendrite length in $\mathrm{R}$. damascena treated group was seen. The R. damascena extract have anti-inflammatory and antioxidant effects in nervous system diseases. Moreover, the essential oil of R. damascena had no anti-inflammatory effect while the hydroalcoholic extract could significantly reduce edema, maybe acted by inhibiting the mediators of acute inflammation. Conclusion: The present review is an effort to provide a detailed scientific literature about pharmacological activities of the plant on nervous system. The R. damascena extracts have antinflammatory and anti-oxidant effects. It can be claimed that these effects have been due to the functions of multifactorial flavonoids, polyphenols and secondary active metabolites.

\section{P92}

The Role of Neuroinflammation in Pathogenesis of Epilepsy

Mahdi Rastekar', Alireza Dehghan', Masoumeh Gholami $^{2}$

${ }^{1}$ Student Research Committee, Torbat Heydariyeh University of Medical Sciences, Torbat Heydariyeh, Iran

${ }^{2}$ Department of Physiology, Faculty of Paramedical Sciences, Torbat Heydariyeh University of Medical Sciences, Torbat Heydariyeh, Iran

The Neuroscience Journal of Shefaye Khatam, 2019; 7(3): P92

Introduction :Inflammation is a biological response of the immune system that can be mediated by different factors, such as pathogens, damaged cells and toxic compounds. These factors may induce acute and/or chronic inflammatory responses in the heart, pancreas, liver, kidney, lung, intestinal tract, reproductive system and brain potentially leading to tissue damage or disease.
Immune and inflammatory reactions occur in brain in various central nervous system (CNS) diseases such as depression, autism, schizophrenia, anxiety and epilepsy. Materials and Methods: In this review abstract, Scopus, PubMed, Science Direct and Google Scholar databases were searched for inflammation, seizure, epilepsy, brain, nervous system, neuroinflammation key words from 2015 to 2018. Results: Studies have shown that neuroinflammation is inflammation of the nervous tissue. It may be initiated in response to a variety of cues, including infection, traumatic brain injury, toxic metabolites, or autoimmunity. Since epilepsy, a neurological disease characterized by recurrent seizures, is often associated with a history of previous lesions in the nervous system. Impaired regulation of the activation and resolution of inflammatory cells and molecules in the injured neuronal tissue such as elevated IL-1 $\beta$, TNF- $\alpha$, IL- 6 transcript levels are critical factors to the development of epilepsy. Also, blood brain barrier breakdown, EP2-COX2 upregulation and TLR pathways are important in this phenomenon. Conclusion: Peripheral and central inflammations allow for the breakdown of the blood-brain barrier due to the upregulation of inflammatory mediators, BBB breakdown permits leukocyte infiltration which generates neuronal hyper-excitability.

\section{P93}

Brain Mitochondrial KATP Channel Properties in a Rat Model of Amyloid- $\beta$ Neurotoxicity: Electrophysiological and Molecular Studies

Maryam Nazari', 2, Javad Fahanik-Babaei', Adele Jafari ${ }^{3}$, Afsaneh Eliassi, ${ }^{1,2}$

${ }^{1}$ Department of Physiology, Faculty of Medical, Shahid Beheshti University of Medical Sciences, Tehran, Iran

${ }^{2}$ Neurophysiology Research Center, Shahid Beheshti University of Medical Sciences, Tehran, Iran

${ }^{3}$ Neurophysiology Research Center, Department of Physiology, Guilan University of Medical Sciences, Rasht, Iran

The Neuroscience Journal of Shefaye Khatam, 2019; 7(3): P93

Introduction: By far, mitochondrial dysfunction considered as a great risk factor for pathogenesis of Alzheimer's disease, but there is a little evidence to elucidate the underlying mechanisms of how the $A \beta$ affects mitochondrial function. Since the mitochondrial potassium channels play a vital role in several mitochondrial functions such as calcium homeostasis, cytoprotection, and apoptosis, a study was conducted to survey whether the electrophysiological and structure of mitochondrial KATP was altered in a rat model of A $\beta$ toxicity. Materials and Methods: $A \beta 1-42(4 \mu \mathrm{g} /$ $\mu \mathrm{l} /$ side) was injected intracerebroventricularly in male Wistar rats (220-250 g). After 14 days, brains were removed, homogenized and then the mitochondrial inner 
membranes were extracted to incorporate in to bilayer lipid membrane (BLM). L- $\alpha$ Phosphatidylcholine was extracted from fresh egg yolk used for BLM formation in a $150 \mu \mathrm{m}$ diameter hole. All recordings were filtered at $1 \mathrm{kHz}$ and stored at a sampling rate of $10 \mathrm{kHz}$ for offline analysis by PClamp10. Western blotting was used to determine the channel structure. Results: In our previous study, single channel recordings revealed a voltage-dependent KATP channel with a slope conductance of $143 \pm 7 \mathrm{pS}$ in 200 cis/50 trans $\mathrm{mM} \mathrm{KCl}$ in control groups. Channel gating were blocked by ATP and glibenclamide (characterize this channel as a KATP channel). Interestingly, in this study, we did not observe any channel activities under $A \beta 1-42$ toxicities. In line with electro-pharmacological observation, western blot analysis shown that there is a $\sim 80 \%, \sim 50 \%$ and $\sim 30 \%$ reduction in Kir6.1, SUR2A and SUR2B subunits expression respectively in $\mathrm{A} \beta$ toxicity compared to control. Conclusion: We have presented the first evidence of a physical and functional alteration of KATP Kir6.1, SUR2A and SUR2B subunits in brain mitochondrial inner membrane under $A \beta 1-42$ toxicity. Current data may help us find more insights into channelopathies in Alzheimer disease and to provide different approaches for disease control.

\section{P94}

\section{Effects of Melissa Officinalis L. Leaves Ethanolic Extract on LPS-Induced Neuroinflammation in Rat Brain}

\section{Amirmohammad Khosrojerdi ${ }^{1}$, Mehran Hosseini ${ }^{2}$}

${ }^{1}$ Student Research Committee, Birjand University of Medical Sciences, Birjand, Iran

${ }^{2}$ Cellular and Molecular Research Center, Department of Anatomy, Birjand University of Medical Sciences, Birjand, Iran

The Neuroscience Journal of Shefaye Khatam, 2019; 7(3): P94

Introduction: During the last decade, lemon balm (Melissa officinalis L.) has attracted an increased interest due to its experimentally proven beneficial effects on many neurological disorders such as cognitive impairment, insomnia, anxiety, and stress. The aim of this study was to elucidate the possible mechanistic inflammatory-modulatory effects of Melissa officinalis $L$. leaves ethanolic extract (MO) on Lipopolysaccharide (LPS)-induced neuroinflammation in rat brain. Materials and Methods: Neuroinflammation was induced by intracerebroventricular (ICV) injection of LPS $(50 \mu \mathrm{g} / 20 \mu \mathrm{l} ; 10 \mu \mathrm{l}$ injected to each ventricle) dissolved in artificial cerebrospinal fluid (CSF) using stereotaxic apparatus in adult male Wistar rats $(n=15)$; whereas, a control group $(\mathrm{n}=5)$ only received artificial CSF (ICV). MO extract at the doses of 50, and $100 \mathrm{mg} / \mathrm{kg}$ was intraperitoneally administrated to the experimental groups $30 \mathrm{~min}$ before LPS injection and continued once per day for 9 days. At the ninth day, the rats were sacrificed, perfused by cold saline through their hearts, and their brains were carefully collected, promptly dissected into cerebral cortex (CC) and hippocampus (HIP). The samples were homogenized, sonicated, centrifuged and their supernatants were collected. The cytokines including tumor necrosis factor alpha (TNF- $\alpha$ ) and Interleukin 1 beta (IL-1 $\beta$ ) were estimated using ELISA kits. Results: Compared to control group, ICV injection of LPS significantly increased TNF- $\alpha$, and IL$1 \beta$ in both of HIP and CC parts. MO treatment (both doses) could effectively normalized TNF- $\alpha$ at both CC and HIP regions. However, MO treatment only at the maximum dose $(100 \mathrm{mg} / \mathrm{kg})$ significantly decreased IL$1 \beta$ just in CC compared to neuroinflammation model group; whereas, it could not significantly ameliorate IL-1 $\beta$ in HIP. Conclusion: To the best of our knowledge, the data of this study for the first time provide evidence that inflammatory-modulatory mechanism may be involve in neuroprotective potential of $\mathrm{MO}$ and could be adhered in further mechanistic studies.

\section{P95}

The Effect of Nanocurcumin and Crocin on Proliferation and Pluripotency of Bone Marrow Derived Mesenchymal Stem: Invitro Study

Nasim Sabooni ${ }^{1,2,3}$, Mojgan Mohammadi, ${ }^{1,}$, Amirreza Boroumand $^{4}$, Jalil Tavakol Afshari ${ }^{1,2}$

${ }^{1}$ Immunology Research Center, Mashhad University of Medical Sciences, Mashhad, Iran

${ }^{2}$ Department of Immunology, Faculty of Medicine, Mashhad University of Medical Sciences, Mashhad, Iran

${ }^{3}$ Student Research Committee, Mashhad University of Medical Sciences, Mashhad, Iran

${ }^{4}$ Department of Neurology, Parnia Stem Cell Institute, Mashhad University of Medical Sciences, Mashhad, Iran

The Neuroscience Journal of Shefaye Khatam, 2019; 7(3): P95

Introduction: Human mesenchymal stem cells (hMSCs) are a multipotent cell that have self-renewal ability, immunomodulatory and neurotrophic effects, make them popular as a therapeutic method for many neurodegenerative diseases such as SCI. The use of synthetic growth factors because of their side effects have been limited to increase the survival and proliferation of MSCs. So natural extractions seem to be good alternative. Curcumin and Cronin are natural Carotenoid they are reported to have various pharmacological properties such anti-inflammatory, anti-tumor, immune and neural cell-growth stimulating effects. Therefore, we examined the effect of Nano curcumin and croc in on bone marrow mesenchymal stem cells (BM-MSCs) proliferation, pluripotency and self-renewal potential in SCI patient in an investor study. Materials and Methods: We use MTT assay and PDT test to evaluate MSC proliferation, 
Flow cytometry assay to measure the percentage of apoptotic MSCs. We also used Real Time PCR method to quantify mRNA expression of proliferation and pluripotency related genes OCT4 and SOX2. Results: our study demonstrated only low concentrations of Nanocurcumin and Cronin were effective for BM-MSC proliferation and increased expression level of OCT4 and SOX2 in treatment groups while high concentrations decreased survival of MSCs and caused to apoptosis compared with untreated group. Conclusion: The results may indicate the beneficial role of Nanocurcumin and crocin in the enhancement proliferation capacity of MSCs and increase pluripotency potential of them via effect on level expression of related genes, although nanocurcumin seem to be more effective than crocin.so this study suggests that using nanocurcumin and crocin separately in culturing of MSCs as an alternative for synthetic growth factors may be useful for cell therapy procedures in SCI patients.

\section{P96}

Comparison of Acupuncture and Medical Therapy on Clinical Symptoms and Electrodiagnostic Changes in Patients with Cervical Disk Herniation

Hamidreza Bahrami-Taghanakil, Hossein Hasanabadi', Hoda Azizi', Mohammadreza Noras ${ }^{3}$, Hasan Jokar', Pegah Bahrami-Taghanaki

'Department of Complementary and Chinese Medicine, Faculty of Traditional and Complementary Medicine, Mashhad University of Medical Science, Mashhad, Iran

${ }^{2}$ Department of Physical Medicine and Rehabilitation, Faculty of Medicine, Mashhad University of Medical Sciences, Mashhad, Iran

${ }^{3}$ Department of Iranian Medicine, Faculty of Traditional and Complementary Medicine, Mashhad University of Medical Sciences, Mashhad, Iran

${ }^{4}$ Department of Internal Medicine, Faculty of Medicine, Mashhad University of Medical Sciences, Mashhad, Iran

${ }^{5}$ Faculty of Medicine, Mashhad University of Medical Sciences, Mashhad, Iran

The Neuroscience Journal of Shefaye Khatam, 2019; 7(3): P96

Introduction: Cervical disk herniation is one of the etiologies for chronic cervical pain which has a significant effect on the quality and efficiency of a patient's life. Access to effective and safe therapeutic protocols are among the main research approaches. In this study the impact of acupuncture and physical activity was studied on the clinical and paraclinical symptoms of such patients. Materials and Methods: In this clinical trial 60 patients were randomly divided into three groups: conventional medical treatment and acupuncture. The 1 st group received $100 \mathrm{mg}$ Celebrex tablets twice daily for 1 month. In the 2 nd group acupuncture was done 3 times a week, 30 min each up to 12 sessions in 1 month.
They were examined at study initiation and 3 months after study termination by electrodiagnostic evaluation and neuromuscular stimulation, and in initiating of study, immediately after the therapies and 3 months after termination of study by questionnaire. The pain index score questionnaire was used for the pain and disability evaluation. The data were analyzed by the ANOVA Test. Results: The two groups were similar in terms of age, sex and educational level. The mean level of pain $(\mathrm{P}=0.38)$, neck stiffness $(\mathrm{P}=0.70)$, murmur and tingling sensation $(\mathrm{P}=0.31)$, restricted neck movements $(\mathrm{P}=0.43)$, and limited daily physical activity $(\mathrm{P}=0.54)$ reduced significantly immediately after treatment in both the acupuncture and medical therapy groups; yet it indicated no significant difference exactly after finishing the therapies. But, the 3-months follow up showed only a significant difference in the mean neck stiffness severity between the two groups $(\mathrm{P}<0.05)$. The findings suggest that the therapeutic response to acupuncture lasts longer and were greater after 3 months. The mean electrodiagnostic indices, CAMP, DML, DSL, MNCV, SNCV and SNAP of the median and ulnar nerves also reduced significantly in the latter mentioned groups after the intervention, but the difference was not remarkable. Conclusion: Both the medical and acupuncture groups improved significantly regarding clinical symptoms; yet it seems that acupuncture was more effective than the conventional treatment alone in reducing the cervical disk herniation symptoms.

\section{P97}

Comparison of the Effect of Four and Eight Weeks of Water Exercises (Hydrotherapy) on Relief of Pain and Muscle Spasm in Children Under Age of 12 with Spinal Cord Injury in Bushehr City

Parviz farzadinia $^{1}$, Aria Salehpour ${ }^{2}$, Alireza Asadnia ${ }^{2}$

${ }^{1}$ Department of Biology and Anatomical Sciences, Faculty of Medicine, Bushehr University of Medical Sciences, Bushehr, Iran ${ }^{2}$ Student Research Committee, Bushehr University of Medical Sciences, Bushehr, Iran

The Neuroscience Journal of Shefaye Khatam, 2019; 7(3): P97

Introduction: Spinal injury is a progressive neurological disorder. due to the sensory-physical, perceptualcognitive and psycho-social disabilities it affects many aspects of working performance. Over 90 percent of children patients experience fatigue. Researchs has shown that water exercise (hydrotherapy) is one of the most effective ways to reduce fatigue, pain and muscle spasms, especially in women. So far, a comprehensive study on the effects of hydrotherapy in children under 12 years old with spinal injury has not been conducted. Materials and Methods :This study was performed in Bushehr aimed to investigate the effects of four and eight weeks of water exercises (Hydrotherapy) on pain 
in children under 12 with spinal cord injury. At present study 40 patient children were divided into two groups, the first group did water exercises for 4 weeks and second group did water exercises for 8 weeks. All children were trained in the water twice a day and each time for an hour under the supervision of a physiotherapist. The results were analyzed using SPSS18 software and tests consisting descriptive statistics, T-test, covariance analysis, and comparison of paired moderated averages. Results: Statistical analyzes showed that patients who had regular water exercise for 8 weeks had a significant effect on pain and muscle spasm relief comparing to 4 weeks' exercise. They also tended to spend more time in water doing exercises without revealing pain or spasms. Conclusion: The issue of improving movement in spinal injuries patients is very essential. Because this disease which was explained above significantly reduces movement and range of motion in the joints due to the pain, spasticity and muscle weakness. By using water exercise individuals tended to stay much longer in the water without expressing pain and spasms.

\section{P98}

Evaluation of the Effect of Pediococcus acidilactici on the Expression of AIM2 Gene in the Demyelinated C57BL/6 Mouse Model

\section{Seyedeh Nikan Nejat, Javad Arasteh, Maryam Tajabadi Ebrahimi}

Department of Biology, Central Tehran Branch, Islamic Azad University, Tehran, Iran

The Neuroscience Journal of Shefaye Khatam, 2019; 7(3): P98

Introduction: Multiple sclerosis is a chronic disease and neurodegenerative disorder that affects the central nervous system and the nerves, leading to the destruction of myelin, which protects the fibers of the neural cells in the brain and spinal cord. One of the important cytosolic DNA sensors is AIM2, which in fact AIM2 is a protein encoded in humans by the AIM2 gene. AIM2 is a member of the Ifi202/IFI16 family. It plays a putative role in tumorigenic reversion and may control cell proliferation. IFN- $\gamma$ induces expression of AIM2. The C-terminal HIN domain binds double-stranded DNA (viral, bacterial, or even host) and acts as a cytosolic dsDNA sensor. This leads to the oligomerization of the inflammasome complex. AIM2 plays an important role in MS by the Th1, Th17 response. The aim of this study was to investigate the effect of Pediococcus acidilactici on the expression of AIM2 gene in demyelinated C57BL/6 mouse model. Materials and Methods: 30 adult female C57BL/6 were divided in to 5 separate groups. First control was Healthy mice group that only they received sterile water by oral gavage to induce stress, second control received $0.2 \%$ cuprizone by oral gavage for 4 weeks, third control group received probiotic with $10^{9} \mathrm{cfu}$ concentration for
8 weeks. Mice in treatment groups were divided in to two groups to survey the prevention and betterment effects of Pediococcus acidilactici. The Prevention group received probiotic with $10^{9} \mathrm{cfu}$ concentration for 2 weeks then cuprizone for 4 weeks. The betterment group received cuprizone for 4 weeks, then probiotic with $10^{9} \mathrm{cfu}$ concentration for 4 weeks. The Luxol Fast Blue staining was used to affirm the existence of demyelination. The quantity of AIM2expression was measured by Real-Time PCR. Results: The results indicate that Pediococcus acidilactici, makes significant difference between the cuprizone control group and treatment groups $(\mathrm{p}=0.000<0.05)$. Conclusion: We verify that Pediococcus acidilactici has potential effects as a novel probiotic on betterment of MS.

\section{P99}

Effects of Nicotinamide in the Protection of Apoptosis and Necrosis in a Stroke

Neshat Ebrahimi, Zahra Farjami, , 3, Negar Khodaeinia ${ }^{3}$, Amirhossein Ashnaei², Zeinab Sadat Hosseini ${ }^{4}$

${ }^{1}$ Laboratory of Cedars-Sinai Medical Center, Los Angles, California, USA

${ }^{2}$ Department of Modern Sciences and Technologies; Faculty of Medicine, Mashhad University of Medical Sciences, Mashhad, Iran

${ }^{3}$ National Research Center of Genetic Engineering and Biotechnology (NIGEB), Department of Medical Genetics, Tehran, Iran

${ }^{4}$ Student Research Committee, Mashhad Branch, Islamic Azad University, Mashhad, Iran

The Neuroscience Journal of Shefaye Khatam, 2019; 7(3): P99

Introduction: Nicotinamide is a form of vitamin B3 which is thought to have a potential role in the treatment of Alzheimer's disease. This substance may have neuroprotective properties, which may protect brain against damage in ischemia-reperfusion. However, the dosage of therapy is not truly clarifying. Materials and Methods: Transient focal cerebral ischemia was persuaded by middle cerebral artery obstruction for $70 \mathrm{~min}$. Several doses of nicotinamide were administered upon reperfusion. Neurological detection scores and infarct volumes were recorded. Results: The scores of neurological deficiency and volumes of infarction were significantly decreased by nicotinamide therapy. Nicotinamide administrated during the first $4 \mathrm{~h}$ of reperfusion can suppress the progress of brain damage. Conclusions: Poly ADP-ribose polymerase (PARP) has a potential effect in repairing the DNA in stroke. Nicotinamide provides energy as a suppressor of PARP. The results propose that initial use of nicotinamide at an appropriate dosage meaningfully improves apoptotic and necrotic brain damage after focal ischemia-reperfusion. 


\section{P100}

Effect of 3-Dimensional Transplantation of Adult Human Brain Derived Neural Stem Cells Seeded in PuraMatrix in Animal Model of Spinal Cord Injury

Sara Abdolahi ${ }^{1,2}$, Azizollah Khodakaram- Tafti ${ }^{1}$, Hadi Aligholi $^{3}$, Ali Gorji ${ }^{2,4,5}$

${ }^{1}$ Department of Pathobiology, Faculty of Veterinary Medicine, Shiraz University, Shiraz, Iran

${ }^{2}$ Shefa Neuroscience Research Center, Khatam Alanbia Hospital, Tehran, Iran

${ }^{3}$ Department of Neuroscience, Faculty of Advanced Medical Sciences and Technologies, Shiraz University of Medical Sciences, Shiraz, Iran

${ }^{4}$ Epilepsy Research Center, Department of Neurology, Westfälische Wilhelms-Universität Münster, Münster, Germany ${ }^{5}$ Department of Neuroscience, Mashhad University of Medical Sciences, Mashhad, Iran

The Neuroscience Journal of Shefaye Khatam, 2019; 7(3): P100

Introduction: A spinal cord injury (SCI) is damage to any part of the spinal cord or nerves at the end of the spinal canal that causes temporary or permanent changes in its function. Therapeutic strategy for SCI includes stabilization surgery, pharmacotherapy and physical therapy. However, there is still no gold standard treatment with sufficient evidence. Inefficiency and adverse effects of standard therapy led to an interest in developing novel therapies for the condition. Stem cellbased therapies hold promise for SCI. Among different cell types, human neural stem cells cultured in selfassembling peptide scaffolds have been suggested as a potential novel method for cell replacement treatment in SCI. Materials and Methods: In this study, we accessed the effects of human neural stem/progenitor cells (hNS/PCs) derived from epileptic human brain seeded in PuraMatrix hydrogel (PM) in an animal model of SCI. In the present study, also we investigated the efficiency of a recombinant lentivirus vector expressing green fluorescent protein (GFP) for genetic engineering of human NS/PCs obtained during brain operation on patients with medically intractable epilepsy. Results: Transplantation of hNS/PCs seeded in PM improved functional recovery, decreased lesion volume and reduced the reactive gliosis at the injury site. Our in vitro study revealed that lentivirus vector expressing GFP efficiently transduced about $80 \%$ of human NS/ PCs. Conclution: The data suggest the transplantation of hNS/PCs cultured in PM as a promising treatment option for cell replacement therapy in SCI.

\section{P101}

Effect of Subdural KCL Application on Trigeminal Nucleus Firing Pattern: A Single Unit Recording Study
Shima Shirzad ${ }^{1}$, Farzaneh Vafaee ${ }^{2}$, Nahid Nasrabadi, Hassan Abbassian ${ }^{2}$

${ }^{1}$ Department of Biology, Faculty of Sciences, Mashhad Branch, Islamic Azad University, Mashhad, Iran

${ }^{2}$ Department of Neuroscience, Faculty of Medicine, Mashhad University of Medical Sciences, Mashhad, Iran

${ }^{3}$ Department of Periodontology, Faculty of Dentistry, Mashhad University of Medical Sciences, Mashhad, Iran

The Neuroscience Journal of Shefaye Khatam, 2019; 7(3): P101

Introduction: Spreading depression (SD) in the brain, plays an important role in some disorders including migraine aura, cerebrovascular diseases, head injury and transient global amnesia. Potassium has a role for $\mathrm{SD}$ in various animal models. It is common to apply high concentrations of $\mathrm{KCl}$ injection for triggering SD. Dural stimulation produces headache-like pain in humans and a well-known pain pathway exists from the dura to the brain via trigeminal nerve and its ganglion. The aim of this study is to investigating changes in the trigeminal nucleus activity pattern due to the use of $\mathrm{KCl}$ solution in subdural space in rat. Materials and Methods :Experiments were performed on 18 adult male rats. For recording EEG, 3 screws on skull, using "elab", Science Beam instruments. apparatus were used. The electroencephalogram was filtered between 1 and $100 \mathrm{~Hz}$ and was continuously monitored via an analog monitor scope. Application of KCL $0.2 \mathrm{ml}$ via subdural injection at 15 minutes of session performed for each rat via a foramen in skull in fronto-parital area and firing rate was recorded for subsequent period of up to 45 minutes. To record single unit activity of trigeminal nucleus a tungsten electrode inserted as stereotaxic coordination $(\mathrm{DV}=8.4, \mathrm{AP}=-8.88, \mathrm{~L}=2.2)$ in same side. Results: The recorded traces showed that when we apply KCL in all cells under recording the frequency and amplitudes were increased significantly. Some change in pattern of firing was seen as ramp increase in amplitudes or frequency and also some burst firing before permanent increase in frequency were seen. Conclusion: Changes in the fairing pattern and frequency of trigeminal nucleus could be a target for therapeutic or research purpose and need to further consideration.

\section{P102}

Rheum Turkestanicum Reduces Glutamate Toxicity in PC12 and N2a Cell Lines

Azar Hosseini', Hamid-Reza Sadeghnial, ${ }^{1,}$ Maliheh Moradzadeh $^{4}$, Arezoo Rajabian ${ }^{1}$

'Pharmacological Research Center of Medicinal Plants, Mashhad University of Medical Sciences, Mashhad, Iran

${ }^{2}$ Department of Pharmacology, Faculty of Medicine, Mashhad University of Medical Sciences, Mashhad, Iran

${ }^{3}$ Neurocognitive Research Center, Faculty of Medicine, Mashhad University of Medical Sciences, Mashhad, Iran 
${ }^{4}$ Golestan Rheumatology Research Center, Golestan University of Medical Sciences, Gorgan, Iran

The Neuroscience Journal of Shefaye Khatam, 2019; 7(3): P102

Introduction: Glutamate, as an important endogenous excitatory neurotransmitter, has major physiological functions in the central nervous system. Nevertheless, glutamate accumulation may result in neuronal damage and cell death under different pathological conditions via various mechanisms, mainly oxidative damage and excitotoxicity. Materials and Methods: In this study, we investigated the neuroprotective effects of Rheum turkestanicum in the glutamate-induced rat pheochromocytoma (PC12 cells) and mouse neuroblastoma (N2a) cell lines. Rutin as an antioxidant was used as positive control. For MTT/ROS and MDA assays, the cells were respectively cultured in 96- and 24-well plates, and for apoptosis assays, the cells were seeded in 24-well plates (100 000 cells/well); it should be noted that all treatments were performed in triplicate. For two hours, the cells were pretreated with only the extract $(6-200 \mu \mathrm{g} / \mathrm{mL})$. Incubation was carried out with the extract for 24 hours, with or without glutamate $(8$ $\mathrm{mM}$ ). Finally, rutin was used to pretreat the cells for two hours, and then, incubation was performed with glutamate for 24 hours. Results: Glutamate cytotoxicity was accompanied by an increment of malondialdehyde (MDA) content, ROS generation and apoptosis induction. However, pretreatment with the root extract of Rheum turkestanicum significantly reduced MDA content, ROS generation and apoptotic cell death. Also rutin at dose of $100 \mu \mathrm{M}$ reduced ROS production and protected against glutamate toxicity. Also the quantification of rutin in R.turkestanicum extract was achieved and was about $0.11 \% \pm 0.01 \mathrm{w} / \mathrm{w}$. Conclusion: All these findings indicated that $\mathrm{R}$. turkestanicum protected $\mathrm{PC} 12$ and $\mathrm{N} 2 \mathrm{a}$ cells against glutamate-induced oxidative cell death and apoptosis and might raise the possibility of R. turkestanicum usage as a neuroprotective agent.

\section{P103}

Investigating the Effect of Lithium on Differentiation of Epidermal Neural Crest Stem Cell into Neural Cells

\section{Shirin Ahmadi, Mohammad Nabiuni, Mohammad} Tahmaseb, Elaheh Amini

Department of Cellular and Molecular Biology, Faculty of Biological Sciences, Kharazmi University, Tehran, Iran

The Neuroscience Journal of Shefaye Khatam, 2019; 7(3): P103

Introduction: Due to the complexity of conditions in neurodegenerative diseases and also key challenge for how to regenerate neurons after injury, combination therapy including cell therapy and drug therapy is important as a new therapeutic strategy. Epidermal neural crest stem cells (EPI-NCSCs) are as one of the best option in cell therapy for various neurological diseases, including spinal cord injury (SCI). In this study, the effect of lithium carbonate with considering of their effects on cellular signaling pathways and neuroprotective/ neurogenesis properties were investigated on expression of neurotrophic factors BDNF, NGF and GDNF in EPI-NCSCs. Materials and Methods: After EPINCSCs isolation of hair follicle bulge and culture cells in collagen-coated culture plates $(1 \mathrm{mg} / \mathrm{ml}$ ) (collagen extracted from rat tail at Kharazmi University).Then, the cells were treated with different concentrations of Lithium carbonate $(0.2,0.4,0.6,0.8,1,1.2,1.4,1.6$, $1.8,2 \mathrm{mM}$ ), after 72 hours trial doses was selected by MTT assay. EPI-NCSCs were treated with selective concentrations for 7 days. During this time, environment of samples was renewed to enhance the effectiveness of the drugs. Then the effect of drug on expression of selective factors was investigated with Real Time PCR. Results: Obtained results indicated an enhancement in the expression of BDNF, NGF and GDNF in treated cells in a dose-dependent pattern $(* \mathrm{p}<0.05, * * \mathrm{p}<0.01$ and $\left.{ }^{* * *} p<0.001\right)$. Conclusion: The results in this study confirmed the neuroprotective/neurogenesis effects of Lithium carbonate, in addition have shown this drug can be used to increase EPI-NCSCs differentiation potential into neural cells in combination therapy of neurodegenerative diseases such as SCI.

\section{P104}

Effects of Propylthiouracil-Induced Hypothyroidism on Learning and Memory in Neonatal and Juvenile Growth in Rats

\section{Saeideh Saadat, 2, Farimah Beheshti, 4, Hassan Azhdari-Zarmehri,}

'Department of Physiology, Faculty of Medicine, Mashhad University of Medical Sciences, Mashhad, Iran

${ }^{2}$ Neurogenic Inflammation Research Center, Mashhad University of Medical Sciences, Mashhad, Iran

${ }^{3}$ Neuroscience Research Center, Torbat Heydariyeh University of Medical Sciences, Torbat Heydariyeh, Iran

${ }^{4}$ Department of Physiology, Faculty of Paramedical Sciences, Torbat Heydariyeh University of Medical Sciences, Torbat Heydariyeh, Iran

The Neuroscience Journal of Shefaye Khatam, 2019; 7(3): P104

Introduction: The role of brain tissues oxidative damage in learning and memory impairments has been well documented. It is also well known that thyroid hormones have a critical role for the brain functions. The purpose of this study was to investigate the role of brain tissues oxidative damage as a possible mechanism of deleterious effects of propylthiouracil (PTU) - induced hypothyroidism on learning and memory in neonatal and juvenile growth in rats. Materials and Methods: 
Fourteen pregnant female Wistar rats were kept in separate cages. After delivery, they were randomly divided into two groups including control and PTU. Rats in the control group received normal drinking water, whereas the second group received drinking water supplemented with $0.05 \%$ PTU from the first day after delivery through the first two months of the life of offspring (the pups of rats). After 60 days, nine male offspring of each group were randomly selected and tested in the Morris water maze (MWM). Then, samples of blood were collected to measure thyroxine. Finally, the brains were removed and total thiol groups and malondialdehyde (MDA) concentrations were determined. Results: Compared to the control group's offspring, serum thyroxine levels in the PTU group's off spring were significantly low $(\mathrm{P}<0.001)$. In MWM, the escape latency and traveled path in the PTU group were significantly higher than that in the control group $(\mathrm{P}<0.01-\mathrm{P}<0.001)$. In $\mathrm{PTU}$ group, the total thiol concentrations in both cortical and hippocampal tissues were significantly lower and MDA concentrations were higher than control group $(\mathrm{P}<0.001)$. Conclusion: It seems that deleterious effect of hypothyroidism during neonatal and juvenile growth on learning and memory is at least in part due to brain tissues oxidative damage.

\section{P105}

Effect of Artemisia Persica Essential Oil on Memory Deficits, Depression and Anxiety in Pentylenetetrazole (PTZ) Kindled Male Mice

\section{Mahbubeh Setorki}

Department of Biology, Izeh Branch, Islamic Azad University, Izeh, Iran

The Neuroscience Journal of Shefaye Khatam, 2019; 7(3): P105

Introcdution: Memory deficits and mood disorders such as depression and anxiety are commonly seen in patients with epilepsy. The aim of this study was to evaluate the effect of Iranian Artemisia essential oil on memory deficits, depression and anxiety in PTZ kindled male mice. Materials and Methods: In this experimental study, 70 male mice were randomly divided into 7 groups. In order to induce seizure, PTZ (35 mg/kg) was injected every other day for 9 day and then at a dose of $60 \mathrm{mg} / \mathrm{kg}$ on the 10th day. Control group received normal saline and treatment groups received essential oil at doses of 50,75 and $100 \mathrm{mg} / \mathrm{kg}$ daily. The positive control group received essential oil $(100 \mathrm{mg} / \mathrm{kg})$ daily and diazepam on the 10th day. Flumazenil group received essential oil $(100 \mathrm{mg} / \mathrm{kg})$ daily and flumazenil on the 10 th day. Results: Artemisia persica essential oil $(50 \mathrm{mg} / \mathrm{kg}$ ) significantly decreased seizure threshold in PTZ- treated mice $(\mathrm{P}<0.05)$. Artemisia persica essential oil at a dose of $50 \mathrm{mg} / \mathrm{kg}$ significantly reduced secondary latency time in the shuttle box test $(\mathrm{P}<0.05)$. Different doses of
Artemisia persica essential oil showed no significant effect on immobility time in the tail-suspension test. The frequency of entry into the closed arms of the elevated plus maze in group treated with $50 \mathrm{mg} / \mathrm{kg}$ of Artemisia persica essential oil was significantly lower than that in PTZ-treated group ( $\mathrm{P}<0.05)$. Conclusion: Artemisia persica essential oil at a dose of $50 \mathrm{mg} / \mathrm{kg}$ effectively reduced seizure threshold, significantly increased the avoidance memory and decreased anxiety in mice receiving PTZ.

\section{P106}

The Effect of Noggin on the Differentiation of Human Amniotic Epithelial Cells into Neuronal Fate

\section{Asma Manzari-Tavakoli', Hassan Niknejad ${ }^{2}$, Ali Moghimi ${ }^{1}$}

${ }^{1}$ Department of Biology, Faculty of Sciences, Ferdowsi University of Mashhad, Mashhad, Iran

${ }^{2}$ Department of Pharmacology, Faculty of Medicine, Shahid Beheshti University of Medical Sciences, Tehran, Iran

The Neuroscience Journal of Shefaye Khatam, 2019; 7(3): P106

Introduction: Stem cells are promising tool for treatment of a variety of diseases such as neurodegenerative diseases (e.g. Spinal cord injury, Multiple Sclerosis, and Parkinson disease). Using proper cell source for this purpose is very important. hAECs, isolated from human amniotic membrane, have favorable and unique characteristics that make them highlighted and attractive for cell therapy. Materials and Methods: In this study, we have investigated the effects of noggin (as BMP antagonist) on differentiation of hAECs (Human Amniotic Epithelial Cells) into Neuronal Fate after 21 days. The amnion layer was mechanically peeled off from the chorion and human amniotic epithelial cells were isolated with trypsin-EDTA in $37^{\circ} \mathrm{C}$. Then, cells were exposured by differentiation medium include noggin (50 and $100 \mathrm{ng} / \mathrm{ml}$ ). After 21 days, cells were fixed and characteristic analysis of AEC-derived Neuronal Fate (neurons) was performed by flow cytometry technique. The following primary antibody was applied for flow cytometer: Map2 (neuron marker). Results: The effect of noggin (BMP antagonist) on MAP-2 expression was confirmed before and after differentiation for 21 days by flow cytometry analysis. The results indicated that noggin increases MAP2 neuronal marker. There are no significant difference between 50 and $100 \mathrm{ng} / \mathrm{ml}$ noggin concentrations. The percentage of MAP2 expression was $74.07 \pm 13.33 \%$ for $50 \mathrm{ng} / \mathrm{ml}$ noggin treated group and $66.82 \pm 4.275 \%$ for the $100 \mathrm{ng} / \mathrm{ml}$ noggin group. Conclusion: Together the results of this study showed that noggin as BMP antagonist acts a critical role in differentiation of hAECs into neuronal fate and hAECs 
were capable of being differentiated to neuronal cells. hAECs is a proper candidate for cell therapy in neural degenerative diseases.

\section{P107}

Strategies for Treatment and Care of Patient with Alzheimer's Disease

\section{Bahareh Zeynalzadeh Ghoochani, Seyed Alireza Derakhshanrad}

Faculty of Rehabilitation Sciences, Shiraz University of Medical Sciences, Shiraz, Iran

The Neuroscience Journal of Shefaye Khatam, 2019; 7(S1): P107

Introduction: Most elderly people with Alzheimer's disease live with family and not in institutions. Because of this, the rehabilitation team needs to include the caregivers and the patient as much as possible in treatment planning. The goal of rehabilitation is to ensure that the patient remains safe, independent, and able to perform ADLs. The planning to reach these goals is best done within the context of the patient's social support system. Materials and Methods: The rehabilitation process begins while the diagnostic workup is still in progress. At this stage of treatment, the rehabilitation plan includes basic training for the patient in performing and adapting the ADLs. It also includes caregiver training and support for significant others so they can make needed environmental modifications to ensure the safety of the patient with dementia. Results: Once the diagnosis is established, treatment planning for longterm care must be carefully made. No matter where the patient will be living, involvement of the caregivers and significant others is essential to maximizing functional outcomes. The emotional, physical, and financial resources of the patient and family must be ascertained. A review of the caretakers' willingness to perform basic tasks or make visits, their willingness to learn and teach the necessary skills, and the realistic need for respites must be determined. Conclusions: The same detailed orientation is needed for institutional staff who care for elderly patients with dementia. The structure and process of care can help patients be maximally active in their self-care and prevent unnecessary anxiety and catastrophic reactions.

\section{P108}

Introduction of a Central ER Stress-Induced Spatial Memory Impairment in Rats: a Model for Studying Alzheimer's Disease

\section{Sahar Askari', Pegah Javadpour ${ }^{1}$, Rasoul Ghasemi ${ }^{2}$}

${ }^{1}$ Department of Physiology, Faculty of Medicine, Shahid Beheshti University of Medical Sciences, Tehran, Iran

${ }^{2}$ Neurophysiology Research Center, Shahid Beheshti University of Medical Sciences, Tehran, Iran
The Neuroscience Journal of Shefaye Khatam, 2019; 7(3): P108

Introduction: Endoplasmic reticulum (ER) is a key cellular organelle involved in many physiological functions. One of the most important ER duties is protein folding. Debility of ER in this duty is known as ER stress. ER stress participates in a wide range of diseases including neurodegeneration diseases. Accumulation of misfolded protein in the ER of neurons is a common feature in Alzheimer's disease (AD) to that extend that $\mathrm{AD}$ is one of the protein misfolding disorders. But so far no model has been designed to make memory impairment similar to AD just by ER stress induction and in previous models of memory impairment, ER stress has been made indirectly. Therefore, the aim of this study was to design a pharmacological AD like memory impairment model by induction of central ER stress. Materials and Methods: In order to induce central ER stress, different doses (5, 10 microgram) of Thapsigargin (TG) were administered to the male Wistar rats through Intracerebroventricular (icv) injection. Cognitive function of animals was evaluated by Morris water maze test. As an indicator for ER stress, hippocampal CHOP protein was assessed by western blotting. Results: The results showed that TG impaired spatial dose-learning and memory in a dose-dependent manner. Meanwhile, molecular results also showed a concurrent increased level of hippocampal CHOP with memory impairment. Conclusion: This study introduces, for the first time, a pure ER stress induced model of memory impairment, which can be used as a basis for future studies on the role of ER stress in AD.

\section{P109}

Effect of Crocus Sativus Extract and Crocin on Oxidative Damage and Inflammation in Streptozotocin-Diabetic Rats

Marzie Ahmadi', Z Rajaei', MA Hadjzadeh', H Nemati $^{1}, \quad M$ Hosseinia ${ }^{1}$

${ }^{1}$ Neurocognitive Research Center, Department of Physiology, Faculty of Medicine, Mashhad University of Medical Sciences, Mashhad, Iran

${ }^{2}$ Department of Physiology, Faculty of Medicine, Isfahan University of Medical Sciences, Isfahan, Iran

The Neuroscience Journal of Shefaye Khatam, 2019; 7(3): P109

Introduction: Diabetes is characterized by high glucose concentrations that lead to an increased production of reactive oxygen species. The resulting oxidative stress can play a key role in diabetes pathogenesis and development of late complications of diabetes, including retinopathy, nephropathy, inflamation, cardiovascular diseases and cognitive disorders. The aim of the present study was to investigate the antihyperglycemic effect, protective potential and memory improvement of the saffron extract and crocin in streptozotocin induced- 
diabetic rats. Materials and Methods: Male Wistar rats $(300 \pm 30 \mathrm{~g})$ were randomly divided into 7 groups, including: control, diabetic (Streptozotocin, $55 \mathrm{mg} / \mathrm{kg}$ ) and diabetics treated intraperitoneally with crocin (at doses of 15,30 and $60 \mathrm{mg} / \mathrm{kg}$ ) or saffron extract (at doses of 50 and $100 \mathrm{mg} / \mathrm{kg}$ ) for 6 weeks. The blood glucose levels were spectrophotometrically measured in all groups at weeks 0 (before diabetes induction), 3 and 6. The levels of thiobarbituric acid reactive substances (TBARS) and total thiol (SH) groups were measured in $10 \%$ liver, kidney and cerebral cortex homogenates at the end of 6 th week. Results: Diabetic rats showed an elevated serum glucose level over those of control rats at weeks 3 and $6(\mathrm{P}<0.001)$. Under our experimental conditions, crocin at dose of $60 \mathrm{mg} / \mathrm{kg}$ and saffron at dose of $100 \mathrm{mg} / \mathrm{kg}$ was found to significantly reduce the blood glucose level in diabetic animals (respectively, $\mathrm{P}<0.05, \mathrm{P}<0.001$ ) at the end of week 6 . In addition, there was a significant increase in TBARS levels in the liver, kidney and cerebral cortex of diabetic animals (respectively, $\mathrm{P}<0.001, \mathrm{P}<0.01, \mathrm{P}<0.005$ ) and decrement in total thiol concentrations in the liver of diabetics $(\mathrm{P}<0.01)$ compared to controls. Treatment of diabetic rats with crocin at doses of 30 and $60 \mathrm{mg} / \mathrm{kg}$ for 6 weeks reduced TBARS content in the liver and kidney (respectively, $\mathrm{P}<0.01, \mathrm{P}<0.001$ ), and crocin at doses of 15 and $30 \mathrm{mg} / \mathrm{kg}$ decreased TBARS levels in the cerebral cortex $(\mathrm{P}<0.01)$ compared to diabetic rats. Treatment of diabetic rats with saffron at doses of 50 and $100 \mathrm{mg} / \mathrm{kg}$ did not change the TBARS and total thiol levels in the tissues compared to diabetics. Conclusions: Our findings suggest that crocin has the hypoglycemic, antiinflamtory properties in streptozotocin-induced diabetes and it can be used with some profit in the treatment of diabetic patients.

\section{P110}

Silymarin-Loaded Chitosan Nanoparticles Attenuate Depressive-Like Behaviors in Rat a Model of Global Cerebral Ischemia/Reperfusion

Seyyed Reza Mokhtari Sangdehi ${ }^{1}$, Akbar Hajizade ${ }^{h}$ Moghaddam $^{1}$, Mojtaba Ranjbar ${ }^{2}$

${ }^{1}$ Department of Biology, Faculty of Basic Sciences, University of Mazandaran, Babolsar, Iran

${ }^{2}$ Faculty of Biotechnology, Amol University of Special Modern Technologies, Amol, Iran

The Neuroscience Journal of Shefaye Khatam, 2019; 7(3): P110

Introduction: Ischemic brain disease is one of the most frequent causes of mortality and disability. Patients who survive global brain ischemia or hypoxia commonly develop cognitive deficit and depression. Chitosan is a family of linear polysaccharides that is used as carrier nanoparticles to transfer drugs to target organs. Silymarin (SM) is one of the polyphenolic antioxidants and extracted from the seeds of Silybum marianum. Despite all medicinal advantage, silymarin has a low bioavailability. Aim of this study was antidepressive effects of silymarin-loaded chitosan nanoparticles (SMCH-NPs) in a rat model of global cerebral ischemia/ reperfusion (I/R). Materials and Methods: The rats were randomly divided into 5 groups (10 rats per group): control; vehicle-treated I/R, $15 \mathrm{mg} / \mathrm{kg}$ aspirin-treated $\mathrm{I} / \mathrm{R} ; 15 \mathrm{mg} / \mathrm{kg}$ SM-treated I/R, $15 \mathrm{mg} / \mathrm{kg}$ SM-CH-NPstreated $\mathrm{I} / \mathrm{R}$. Cerebral I/R injury rats were induced by bilateral common carotid artery occlusion (BCCAO) for $10 \mathrm{~min}$ followed by reperfusion. Aspirin, SM and SM-CH-NPs were oral administrated to the rats 14 days before I/R induction. 24 hours after I/R induction, all rats were subjected to a forced swimming test to determine depression. Results: Our study showed a significant increase and decrease in the immobility and climbing times in I/R damage group compared with the control group $(p<0.01)$. SM and SM-CH-NPs treatment reversed depression-like behaviors to the normal level. Conclusion: Taken together these data demonstrate that SM and SM-CH-NPs attenuates the depressive like behavioral induced by global cerebral ischemia in rat.

\section{P111}

Effects of Peripheral Lipopolysaccharide Administration on Spatial Memory and Passive Avoidance Learning in Rats

Amir Reza Beheshti', Motahareh Rouhi Ardeshiri², Esmaeil Akbari ${ }^{2}$

${ }^{1}$ Faculty of Medicine, Mazandaran University of Medical Sciences, Sari, Iran

${ }^{2}$ Department of Physiology, School of Medicine, Mazandaran University of Medical Sciences, Sari, Iran

The Neuroscience Journal of Shefaye Khatam, 2019; 7(3): P111

Introduction: Neuro-inflammation, is one of an early events in the pathogenesis of Alzheimer's disease (AD) as well as loss of synapses, microglial activation, and accumulation of amyloid beta $(A \beta)$. Stimulating the immune responses in the brain and releasing inflammatory mediators cause to generate oxidative stress agents. Lipopolysaccharide (LPS), a toxic part of gram negative bacterial cells which are found in the outer membrane of these cells, has been classically proposed as trigger for immune system to release the pro-inflammatory cytokines such as TNF- $\alpha$, IL- $1 \beta$, and IL-6. The purpose of this study was to assess, the effects of LPS on memory impairment in rats. Then, oxidative stress biomarkers in both cortex and hippocampal formation were evaluated. Materials and Methods: The experimental animals were described as: (1) control, (2) LPS. In order to measure the biochemical markers, the brain tissues were removed. ( $\mathrm{n}=10$ in each group). Results: Time spent in target quadrant in the Morris 
water maze task and the delay time to enter the dark part in the passive avoidance learning task were significantly shorter in LPS treated group compared to the control group. The concentration of Malondialdehyde (MDA), in the cortex and hippocampus of the LPS treated rats significantly increased compared to the control group. The total content of the thiol group, the activities of catalase (CAT) and superoxide dismutase (SOD) in cortical and hippocampal tissues of LPS treated rats decreased regarding to the control group. Conclusion: These results indicated that LPS could disturb the brain's immune system towards memory destruction by increasing oxidative stress biomarkers.

\section{P112}

Effects of Cholestasis on Passive Avoidance Memory in Bile Duct Ligated Male Rats: Role of Opioidergic Systems

\section{Maryam Moghimian, Mahsa Pureidi, Seyed Hosein Abtahi Ivary, Masoumeh Fani}

Department of Basic Sciences, Faculty of Medicine, Gonabad University of Medical Sciences, Gonabad, Iran

The Neuroscience Journal of Shefaye Khatam, 2019; 7(3): P112

Introduction: Memory is impaired in patients with liver disease. Opioidergic systems are known to be involved in cholestasis. The aim of this study was to investigate the possible effect of this system in BDLinduced memory formation in the male rat. This study investigated the impact of cholestasis progression on the acquisition and retention times in the passive avoidance test. Materials and Methods: Cholestasis was induced in male Wistar rats by ligating the main bile duct. Male rats divided into none operated controls, sham-operated, and BDL groups. Memory was assessed by the passive avoidance learning test at day 7 , day 14 , and day 21 post-bile duct ligation. The serum levels of bilirubin, alanine aminotransferase, and alkaline phosphatase were measured. Results: The results showed initial latency was not affected till 21 post-bile duct ligation whereas decreases memory retrieval 7, 14 and 21 days post BDL. Therefore, short-term memory was affected in the early stage of cholestasis. Intraperitoneal injection of naloxone ( 0.6 and $0.8 \mathrm{mg} / \mathrm{kg})$ ), $30 \mathrm{~min}$ before testing, 7 days after bile duct ligation did not show a significantly altered in memory acquisition, whereas injection of morphine $(4 \mathrm{mg} / \mathrm{kg}$ and $6 \mathrm{mg} / \mathrm{kg}) 30 \mathrm{~min}$ before testing 7 days after bile duct ligation, showed a significantly increased in acquisition time. Both doses of morphine could restore cholestasis - induced amnesia in stepthrough passive avoidance test. Also, injection of a dose of naloxone $(0.6 \mathrm{mg} / \mathrm{kg}) 15 \mathrm{~min}$ before the injection of a dose of morphine $(6 \mathrm{mg} / \mathrm{kg})$ exerted no effect on memory acquisition in the 7 days post-BDL rat. Conclusion: The result show impaired of memory happens in the early stage of cholestasis that injection of pre-test morphine caused the facilitation of memory retrieval. There also appears to be an effect of the opioidergic system on memory acquisition in the cholestatic male rat.

\section{P113}

Dextran- Curcumin Downregulates Tumor Necrosis Factor- $\alpha$ and Interleukin-6 Expression and Protects Rat Brains Against Global Ischemia

Homa Talebi', Akbar Hajizadeh Moghaddam ${ }^{1}$, Mahbobe Zare ${ }^{2}$, Mojtaba Ranjbar ${ }^{2}$ Sedigheh Khanjani Jelodar ${ }^{3}$

${ }^{1}$ Department of Biology, Faculty of Basic Sciences, University of Mazandaran, Babolsar, Iran

${ }^{2}$ Faculty of Herbs, Amol University of Special Modern Technologies, Amol, Iran

${ }^{3}$ Faculty of Biology, Shahid Beheshti University, Tehran, Iran

The Neuroscience Journal of Shefaye Khatam, 2019; 7(3): P113

Introduction: Stroke is neurodegenerative disease with pathophysiological cascade, such as oxidative stress and inflammatory event. The inflammatory response following ischemic stroke with pathological features such as necrotic cells and increased reactive oxygen species, tumor necrosis factor- $\alpha(\mathrm{TNF}-\alpha)$, interleukin$1 \beta$ (IL-1ß) and interleukin-6 (IL-6), by a variety of activated cell types and expand an infarct initiated by ischemic insult. Curcumin, a natural phenolic compound, has been pharmacological effects such as anti-inflammatory, antioxidant and anti-apoptosis. The loss of water-solubility and its low absorption inside the body include problems with the use of curcumin. So recently by various novel drug delivery methods improved its bioavailability and absorption. Dextran is a highly biocompatible and biodegradable neutral bacterial exopolysaccharide with simple repeating glucose subunits with highly suitable as nanomedicine, nanodrug. Most importantly, it is extremely water soluble and shows no post drug delivery cellular toxicity. The purpose of this study was to investigate the anti-inflammatory effects of Dextran-curcumin conjugate in animal model of ischemia. Materials and Methods: pre-treatment group, was treated oral administration with curcumin or dextran- curcumin for 30 days and vehicle group received distilated water. For induction of ischemic model, rats were anaesthetized and carotid arteries were selected and clamped for 5 min (time of ischemia), There after the vascular clamps were removed for the next $10 \mathrm{~min}$ (time of reperfusion), and both carotid arteries were clamped again for $5 \mathrm{~min}$. In order to investigate the anti-inflammatory effects of Dextran- curcumin conjugate, expression of IL-6 and TNF- $\alpha$ gene was performed using RT-PCR method. Results: Our study indicated that TNF $\alpha$ and IL-6 levels increace in ischemic groupe and dextran curcumin 
conjugate Improve effects after ischemic stroke $(\mathrm{p}<$ 0.01). Conclusion: According to our research, Dextrancurcumin conjugate has protective effects on ischemia stroke.

\section{P114}

Impact of Transcranial Direct Current Electrical Stimulation on Two Patients with Parkinson's Disease

Hamid Mirhosseini ${ }^{1}, \quad$ Reza Bidaki ${ }^{1}, \quad$ Zahra Torkashvand ${ }^{2}$, Marzie Abutorabi-Zarchi', Nahid $\operatorname{Zare}^{4}$

${ }^{1}$ Research Center of Addiction and Behavioral Sciences, Shahid Sadoughi University of Medical Sciences, Yazd, Iran

${ }^{2}$ Department of Surgical Technologies and Anesthesiology, Shahid Sadoughi University of Medical Sciences, Yazd, Iran ${ }^{3}$ Department of Neurology, Shahid Sadoughi University of Medical Sciences, Yazd, Iran

${ }^{4}$ Department of Occupational Therapy, University of Social Welfare and Rehabilitation Sciences, Tehran, Iran

The Neuroscience Journal of Shefaye Khatam, 2019; 7(3): P114

Introduction: The motor function is associated with the activity of both the motor and prefrontal cortices. The efficacy of Transcranial Direct Current Stimulation (tDCS) over specific brain cortices has been examined in many psychiatric and neurologic disorders. The aim of this study is report of the TDCS effects on two female of advanced age, with idiopathic Parkinson's disease (PD). Materials and Methods: We considered 50-minute sessions of bi-lateral primary motor cortices as well as left DLPFC anodal stimulation using tDCS with passive stretching exercises simultaneously for a total of 20 sessions in 7 weeks. Clinical signs and EEG waveform were assessed at distinct times. Results: Both of the two patients showed improvement in motor function for a short time. EEG changes to some extent were in relation to clinical states. Conclusion: It seems that tDCS can be an auxiliary treatment for motor dysfunction in Parkinson's disease, however, further studies must be carried out in order to prove the claim

\section{P115}

Effects of Chronic Uncontrolled Hyperglycemia on Ultrastructure of Hippocampus Astrocytes

\section{Shahriar Ahmad Poor', Elham Hemati Zade ${ }^{2}$}

${ }^{1}$ Department of Anatomy, North Khorasan University of Medical Sciences, Bojnourd, Iran

${ }^{2}$ North Khorasan University of Medical Sciences, Bojnourd, Iran

The Neuroscience Journal of Shefaye Khatam, 2019; 7(3): P115

Introduction: Altered function of astrocytes has been noticed in diabetes mellitus type1. The aim of our study was to determine the effects of chronic uncontrolled hyperglycemia on ultrastructure of hippocampus astrocytes. Materials and Methods: Experimental diabetes was induced in adult male wistar rats by application of streptozotocin $(60 \mathrm{mg} / \mathrm{kg})$. Eight weeks after application of streptozotocin, the hippocampi were removed and the number of astrocytes and degenerated neurons were studied using transmission electron microscopy. Results: The astrocytes exhibited structural changes, including reduced electron density of the nucleus, electro-lucent heterochromatin, and vacuolated cytoplasm. The mean number of astrocytes in diabetic group significantly increased compared to the controls. Conclusion: Our data revealed that chronic diabetes mellitus type 1 is associated with increase in the mean number of astrocytes and ultrastructural astrocyte changes in the hippocampus.

\section{P116}

91Fingolimod Improves the Functional Recovery of Optic Pathway and Alleviates the Expression Level of Histone Deacetylase in Focal Demyelination Model of Rat's Optic Chiasm

Mona Hashemian 1, 2, Maryam Ghasemi-Kasman ${ }^{3,4}$, Hadi Parsian', 3, Farzin Sadeghi, 5

${ }^{1}$ Student Research Committee, Babol University of Medical Sciences, Babol, Iran

${ }^{2}$ Department of Clinical Biochemistry, Faculty of Medicine, Babol University of Medical Sciences, Babol, Iran

${ }^{3}$ Cellular and Molecular Biology Research Center, Health Research Institute, Babol University of Medical Sciences, Babol, Iran

${ }^{4}$ Neuroscience Research Center, Health Research Institute, Babol University of Medical Sciences, Babol, Iran

${ }^{5}$ Department of Medical Microbiology, Faculty of Medicine, Babol University of Medical Sciences, Babol, Iran

The Neuroscience Journal of Shefaye Khatam, 2019; 7(3): P116

Introduction:Fingolimod (FTY720) as a sphingosine 1-phosphate (S1P) receptor agonist, has been introduced as the first oral medicine for relapsing-remitting multiple sclerosis (MS). Besides immunomodulatory role, FTY720 exerts beneficial effects on remyelination process in central nervous system (CNS). In this study, the effect of FTY720 on conductivity of visual signals, myelin repair, glial activation and expression levels of histone deacetylase 1 (HDAC1)/ S1P1R has been evaluated in lysolecithin (LPC)-induced demyelination model in optic chiasm. Materials and Methods: In order to induce demyelination model, LPC $(1 \%, 2 \mu \mathrm{l})$ was injected into rat's optic chiasm. Visual evoked potential recording (VEP) was used to measure the latency of visual waves. The extent of demyelination area and levels of glial activation were assessed using immunostaining. Gene expression analysis was performed to evaluate the expression levels of HDAC1, S1P1Rand Olig2 in the optic chiasm. Results: Analysis 
of electrophysiological data showed that injection of LPC increased the latency of visual signals and application of FTY720 significantly improved the functional recovery of visual pathway and alleviated the levels of glial activation in the optic chiasm. FTY720 enhanced the myelin repair and upregulated the expression levels of Olig2. Additionally, the expression levels of HDAC1/ S1P1R were significantly reduced in animals treated with FTY720. Conclusion: Cumulatively, the results of the present study demonstrate that FTY720 application improves the functional recovery of optic pathway through enhancement of remyelination, alleviation of glial activation and downregulation of S1P1R/HDAC1.

\section{P117}

The Serum Mineral Level: Mediatory Role on Discharge Disability in Stroke Patients, North of Iran

Ali Janahmadiahangar', Soraya Khafri', Payam Saadat, Shayan Alijanpour', Mansor Babaei, Mohammadali Bayani, Alireza Firouzjahi ${ }^{4}$, Farshad Fadaee Jouybari, Sepideh Hosseini Shirvani, Fatemeh Frajzadeh ${ }^{4}$

${ }^{\prime}$ Mobility Impairment Research Center, Babol University of Medical Sciences, Babol, Iran

${ }^{2}$ Department of Biostatistics and Epidemiology, Babol University of Medical Sciences, Babol, Iran

${ }^{3}$ Student Research Committee, Isfahan University of Medical Sciences, Isfahan, Iran

${ }^{4}$ Babol University of Medical Sciences, Babol, Iran

The Neuroscience Journal of Shefaye Khatam, 2019; 7(3): P117

Introduction: stroke is one of the most leading cause of mortality and disabilities associated with neurological disorders. Due to less availability of data on the associations between mineral serum level and stroke, this study was conducted. Materials and Methods: This cross-section analytical study on stroke patients referred to the Ayatollah Rouhani Hospital of Babol over one year conducted. Demographic characteristic of patients, risk factors, types, admit score, and discharge scale of stroke were determined and mineral serum level of patients measured. A path analysis and logistic regression in spss v23 used. Results: Of 216 stroke patients, and $185 \mathrm{cases}$ $(85.6 \%)$ were ischemic. The discharge scale of 138 cases (64.4\%) mild, 49 cases (22.7\%) moderate and 29 cases $(12.9 \%)$ were sever or death. Patients with moderate and severe admit score, with hemorrhagic stroke type, hypertension and diabetes mellitus and lives in village have significantly more discharge scale. Low serum level of Magnesium and Calcium statistically significant with discharge disability status. Logistic regression modeling result for predicting H.T.N, ischemic type and discharge scale in studied patients. About H.T.N, Ca, age older than 60 were the effective factors and about ischemic type; I.H.D, $\mathrm{Mg}$ and $\mathrm{Ca}$ were effective factors
.In addition, $\mathrm{Mg}, \mathrm{H} . \mathrm{T}$. $\mathrm{N}$, admit score and ischemic type were the predictors of the discharge scale. Conclusion: Hypomagnesaemia play role in discharge disability status, this new clue can be effective in determination of prognosis and in some aspects of therapeutic measures.

\section{P118}

Alteration Level of Hippocampus BDNF Expression and Long Term Potentiation Following Microinjection 5-HT1F Receptor Agonist in the Reinstatement of Methamphetamine Behavior

Reihaneh Sadeghian', Siamak Shahidi', Alireza Komaki', Sara Soleimani Asl ${ }^{2}$

${ }^{1}$ Neurophysiology Research Center, Hamadan University of Medical Sciences, Hamadan, Iran

${ }^{2}$ Department of Anatomy, Faculty of Medicine, Hamadan University of Medical Sciences, Hamadan, Iran

The Neuroscience Journal of Shefaye Khatam, 2019; 7(3): P118

Introduction: Methamphetamine's (METH) reinstatement affects the function of the serotonergic system that it is important for brain-derived neurotrophic factor (BDNF) level and synaptic plasticity. At the moment, the physiological function of 5-HT1F receptor is unknown although the distribution of this receptor in the memory and reward regions is known. Herein, agonist effects of this receptor (LY 344864) on behavior, longterm potentiation (LTP) and BDNF level in reinstated METH-rats were assessed. Materials and Methods: Conditioned place preference induced by the injection of METH $(5 \mathrm{mg} / \mathrm{kg}$; i.p) or saline on the conditioning days. On the last day of extinction, rats obtained METH [Simultaneously with LY $(2 \mu \mathrm{g} / 5 \mu \mathrm{l}$; i.c.v $)$ or vehicle]. In control or sham groups, rats received Saline or Saline +vehicle instead methamphetamine, respectively. On the reinstatement day, preference scores were calculated. Then, electrophysiology and western blotting methods were performed for measurements LTP components, and expression of BDNF, respectively. Results: The results showed the conditioning scores of priming METH treatment significantly increased more than other groups and pre-treatment with LY significantly reduced the reinstatement of priming METH seeking. Furthermore, PS amplitude and EPSP slope in vehicle +priming METH rats were more than others. Also, PS amplitude in LY + METH group was lower than priming METH group. Likewise, BDNF expression considerably increased in METH group although treatment with LY was no different to sham group. Conclusion: These results suggest serotoninergic system plays regulatory roles in synaptic plasticity and there is the vital effect of synergism among these systems in METH-reinstatement behaviors.

\section{P119}


The Effects of Olibanum and Its Constituents on Memory Impairement Induced by Lipopolysaccharide in Rats

Narges Marefati ${ }^{1,2}$, Mahmoud Hosseini, ${ }^{1,2}$

${ }^{1}$ Neurogenic Inflammation Research Center, Department of Physiology, Faculty of Medicine, Mashhad University of Medical Sciences, Mashhad, Iran

${ }^{2}$ Neurocognitive Research Center, Department of Physiology, Faculty of Medicine, Mashhad University of Medical Sciences, Mashhad, Iran

The Neuroscience Journal of Shefaye Khatam, 2019; 7(3): P119

Introduction: Since ancient times, the therapeutic properties of resinous of Boswellia serrata were known. The resin obtained from the bark of trees are known as frankincense, olibanum or incense. More than 200 constituents have been known in these resin that can responsible for therapeutic properties in various inflammatory disease. Meanwhile, boswell Acid and incensole Acetate have distinct anti-inflammatory properties. Materials and Methods: 40 rats were divided into 6 groups $(\mathrm{n}=10)$ : Control, LPS, boswellic acid 5 (BA 5), BA 10, incensole acetate 2.5 (IA 2.5) and IA 5. For 6 days, the animals received $1 \mathrm{mg} / \mathrm{kg}$ LPS (i.p) with extract solventor (DMSO-saline), LPS along with treatments or DMSO and saline without LPS injection. LPS was also administered two $h$ before the behavioral experiments and treatments were injected 30 minutes before LPS. Animals were tested with Morris Water maze (MWM) and Passive avoidance tests. In the last day, the hippocampus were removed. Results: LPS administration increased the duration and distance to find the platform in the MWM test in compare to control group in 5 days $(\mathrm{P}<0.05$ to $\mathrm{P}<0.001)$. While, LPS decreased the latency to enter to the dark compartment after receive the sock in PA $(\mathrm{P}<0.001)$. Pretreatment with the both two doses of all treatments enhanced performances of the rats in MWM $(\mathrm{P}<0.05$ to $\mathrm{P}<0.01)$ and $\mathrm{PA}$ test $(\mathrm{P}<0.01$ to $\mathrm{P}<0.001)$. LPS also increased hippocampal IL-6, TNF- $\alpha$, NO, GFAP and MDA levels $(\mathrm{P}<0.001)$ while, decreased IL-10, BDNF, CAT, SOD and thiol $(\mathrm{P}<0.001)$. All treatments increased hippocampal IL-10, BDNF, CAT, SOD and thiol groups $(\mathrm{P}<0.01$ to $\mathrm{P}<0.001)$. Conclusion: We found for the first time that these resin and its constituents could attenuated the generation of pro-inflammatory, oxidant agents and reinforce the anti-inflammatory cytokines and anti-oxidant agents and improve memory impairment induced by LPS.

\section{P120}

The Role of Nitric Oxide in the Dorsolateral Periaqueductal Grey in the Central Regulation of Cardiovascular Activity in Rat

Amirsadra Zangouei ${ }^{1}$, Reza Shahrokhnezhad ${ }^{1}$, Reza

\section{Mohebbati ${ }^{2}$, Mohammad Naser Shafei ${ }^{3}$}

${ }^{1}$ Student Research Committee, Faculty of Medicine, Mashhad University of Medical Sciences, Mashhad, Iran

${ }^{2}$ Department of Physiology, Faculty of Medicine, Mashhad University of Medical Sciences, Mashhad, Iran

${ }^{3}$ Neurogenic Inflammation Research Center, Mashhad University of Medical Sciences, Mashhad, Iran

The Neuroscience Journal of Shefaye Khatam, 2019; 7(3): P120

Introduction: Nitric oxide (NO) is an important neurotransmitter in the central nervous system which is involved in different processes like cardiovascular regulation. The presence of nitrergic neurons in the dorsolateral periaqueductal grey (dlPAG) has been determined. However, the impact of the nitrergic system of dIPAG in central cardiovascular regulation is unknown. In the current study, we aimed to evaluate the cardiovascular responses by microinjecting NGnitro-L-arginine methyl ester (L-NAME) (an inhibitor of NO synthase) and L-arginine (L-Arg) (a precursor of NO) into dlPAG. Materials and Methods: Eighteen male Wistar rats were divided into 3 groups: 1) Control, 2) L-NAME (90 nmol), and 3) L-Arg (60nmol). After anesthesia with urethane $(1.5 \mathrm{~g} / \mathrm{kg}$, i.p), the femoral artery was isolated and cannulated with angiocath. The skull was drilled using stereotaxy over dlPAG. The considered drugs were microinjected into the dlPAG using a micropipette. The cardiovascular parameters including systolic blood pressure (SBP), mean arterial pressure (MAP), and heart rate (HR) were evaluated by Lab Chart software. The data was obtained and comparison was performed by repeated measures ANOVA test. Results: Our findings showed that the HR in the L-Arg group significantly decreased and in the L-NAME group significantly increased as compared to the control group. In addition, the vascular parameters including SBP and MAP meaningfully increased in the considered groups in comparison with the control group. Conclusion: According to our results, we suggest that the nitrergic neurons of the dlPAG is involved in central cardiovascular regulation.

\section{P121}

Bone Marrow Stem Cell Therapy in Combination with Caffeic Acid Phenethyl Ester Improves Manifestations of Parkinson Disease in Rat Models

Manoochehr Safari', Zahra Pakfetrat ${ }^{2}$, Sam Zarbakhsh ${ }^{1}$ Khojaste Rahimi Jaberi ${ }^{2}$

${ }^{1}$ Research Center of Nervous System Stem Cell, Department of Anatomy, Semnan University of Medical Sciences, Semnan, Iran ${ }^{2}$ Shiraz Nephro-Urology Research Center, Shiraz University of Medical Sciences, Shiraz, Iran

The Neuroscience Journal of Shefaye Khatam, 2019; 7(3): P121

Introduction: Parkinson's disease (PD) is a progressive 
neurodegenerative motor control disorder and the second most debilitating disease of the nervous system, this disease mainly characterized by four specific symptoms: hands and feet tremor in rest, slowness of movement or bradykinesia, poor balance and stiffness in limbs. Propolis is a waxy substance produced by bees. It has anti-bacterial, anti-fungal, anti-parasitic, antioxidant and anti-inflammatory properties. Caffeic acid phenethyl ester (CAPE) is one of Propolis compounds which exerts an antioxidant compound. Stem cells have the capability to self-renew and differentiate to all types of cells, including blood, nervous and cartilage cells. Materials and Methods: In this study, the effect of CAPE and bone marrow stem cells in rat model of Parkinson disease were evaluated. Adult male albino Wistar rat weighing 200$250 \mathrm{~g}$ were procured from the animal center of Semnan University of Medical Sciences. Rats were randomly divided into 6 groups $(n=7)$. Rats were treated by intranasal administration of 1-methyl-4-phenyl-1, 2, 3, 6-tetrahydropyridine (MPTP). In this study PD rats were examined against the therapeutic groups that received CAPE and bone marrow stem cells. Results: Our study confirmed that intranasal administration of MPTP cause dopaminergic neuron reduction in substantia nigra. By intra peritoneal administration of CAPE $(10 \mu \mathrm{M})$ and injection of $2 \times$ bone marrow stem cell, number of dopaminergic neuron increased, number of apoptotic neurons decreased and behavioral symptoms of rats were improved. Conclusions: Our data provide evidence, that bone marrow stem cells and pretreatment with CAPE, is beneficial treatment for Parkinson disease.

\section{$\mathbf{P} 122$}

Neuroprotective Effects of Imipramine Against Memory Impairment and Caspase-3 Cleavage in STZ Induced Alzheimer's Disease

\section{Pegah Javadpour', Sahar Askari', Leila Dargahi',} Abolhassan Ahmadiani', Rasoul Ghasemi ${ }^{1,4}$

\author{
${ }^{1}$ Department of Physiology, Faculty of Medicine, Shahid Beheshti \\ University of Medical Sciences, Tehran, Iran \\ ${ }^{2}$ Neurobiology Research Center, Shahid Beheshti University of \\ Medical Sciences, Tehran, Iran \\ ${ }^{3}$ Neuroscience Research Center, Shahid Beheshti University of \\ Medical Sciences, Tehran, Iran \\ ${ }^{4}$ Neurophysiology Research Center, Shahid Beheshti University \\ of Medical Sciences, Tehran, Iran
}

The Neuroscience Journal of Shefaye Khatam, 2019; 7(3): P122

Introduction: Dysregulation in the metabolism of sphingolipids has been reported in various diseases, including Alzheimer's disease (AD). Ceramides are one of the most important bioactive members of this family that an increase in its amount was detected in AD brain. Tricyclic antidepressants (TCAs) are a class of medications which negatively regulate Ceramide levels.
So far, there is no study about the effect of these drugs on memory impairment and apoptosis in STZ-induced AD model. Materials and Methods: In this study, male wistar rats were administrated STZ intracerebroventricularly (icv, day 0 and day 2) and treated with intraperitoneal (ip) injection of imipramine (10 and $20 \mathrm{mg} / \mathrm{kg}$ ) for 14 consecutive days. Then spatial learning and memory were assessed by Morris water maze on days 15-18. After that, the hippocampi of rats were isolated and the amount of the cleaved caspase-3 was analyzed using western blotting. Results: The results showed that while STZ administration caused memory impairment, the treatment of animals with imipramine could prevent memory impairment dose-dependently. Furthermore, at the molecular level, imipramine reduced the amount of cleaved caspase-3. Conclusion: Our findings, for the first time, showed that imipramine has effects other than previous known effects including memory improvement. Since imipramine can inhibit ceramide synthesis, these results imply that normalized ceramide level might play role in such neuroprotective effects.

\section{P123}

\section{Nimodipine Promotes Regeneration of Peripheral} Nerve

\section{Arash Abdolmaleki ${ }^{1,2}$}

${ }^{1}$ Department of Engineering Sciences, Faculty of Advanced Technologies, University of Mohaghegh Ardabili, Namin, Iran ${ }^{2}$ Bio Science and Biotechnology Research Center (BBRC), Sabalan University of Advanced Technologies (SUAT), Namin, Iran

The Neuroscience Journal of Shefaye Khatam, 2019; 7(3): P123

Introduction: Acellular nerve graft is an alternative to autograft for the repair of short gaps associated with peripheral nerve injury. It provides a suitable threedimensional structure that supports and guides axonal regeneration. However, outcomes associated with the use of acellular nerve grafting are often inferior to those achieved with autografts, particularly over long lesion gaps. Therefore, this experimental study was conducted to evaluate the effects of nimodipine on the efficacy of acellular nerve allografts seeded with bone marrow stem cells (BMSCs) to bridge a $15-\mathrm{mm}$ sciatic nerve gap. Materials and Methods: Sixty rats were randomly allocated into six groups ( $\mathrm{n}=10$ per group), including the healthy control group, sham surgery group, autograft group, acellular nerve scaffold (ANS) group, ANS + BMSCs group and ANS + BMSCs + 10 $\mathrm{mg} / \mathrm{kg}$ nimodipine group. The experimental groups was treated daily with nimodipine at the dose of $10 \mathrm{mg} / \mathrm{kg}$ for two weeks. After implantation, motor function was assessed and electrophysiological, histomorphometry, and molecular tests were performed. Results: Animals treated with nimodipine immediately after implantation 
showed significantly better regeneration and motor function outcome compared with ANS group, and ANS + BMSCs group. No significant difference was observed between the nimodipine treatment group and the autograft group. Conclusion: These findings suggest that nimodipine treatment resulted in improved regenerative properties of cell-seeded nerve allografts, likely via increasing the viability and retention of transplanted BMSCs.

\section{P124}

Nursing Intervention and Self-Care Ability of Stroke Survivors, Isfahan, Iran

\section{Nasrollah Alimohammadi ${ }^{1}$, Naireh Vahid Dastjerdi, Saieed Pahlevan Zadeh ${ }^{3}$, Shayan Alijanpour ${ }^{4,5}$}

${ }^{1}$ Nursing and Midwifery Care Research Center, Faculty of Nursing and Midwifery, Isfahan University of Medical Sciences, Isfahan, Iran

${ }^{2}$ Critical Care Nursing, Faculty of Nursing and Midwifery, Isfahan University of Medical Sciences, Isfahan, Iran

${ }^{3}$ Department of Psychiatric Nursing, Faculty of Nursing and Midwifery, Isfahan university of Medical Sciences, Isfahan, Iran ${ }^{4}$ Critical Care Nursing, Students Research Committee, Faculty of Nursing and Midwifery, Isfahan University of Medical Sciences, Isfahan, Iran

${ }^{5}$ Pre-Hospital Emergency Organization and Emergency Medical Service Center, Babol University of Medical Sciences, Babol, Iran

The Neuroscience Journal of Shefaye Khatam, 2019; 7(3): P124

Introduction: There is limited data on identifying the factors affecting the perceived self-care ability of stroke survivor. Therefore, the current study was conducted to identify the impact of nursing intervention about selfefficacy on self-care ability. Materials and Methods: This clinical trial which includes three stages with a test plan before, immediately after and one month after stroke occurrence in Isfahan. Stroke survivors were selected by the simple continuous method and randomized in the intervention ( 5 groups with 5 cases with 6 sessions intervention) and control groups. Then, Mini-mental state examination and Barthel questionnaire were completed for both groups before, after intervention and one month after. then data were analyzed by Chi-square, t-test and Man-Whitney test in SPSS version 23. Results: Fifty stroke survivor equally divided into the intervention and control groups. The difference in mean scores of self-care before the intervention was not significant between two groups $(52 \pm 6.6$ intervention group vs. $53.8 \pm 5.5$ control group, $\mathrm{P}=0.30)$ but immediately $(63.8 \pm 14.8$ intervention group vs. $54.6 \pm 12.1$ control group, $\mathrm{p}=0.02$ ) and 1 month after the intervention $(90.4 \pm 16.1$ intervention group vs. $59.2 \pm 13.9$ control group, $\mathrm{P}<0.001)$ was significant. the mean scores of selfcare patients in the intervention group $(52 \pm 6.6$ before,
$63.8 \pm 14.8$ after the intervention, $90.4 \pm 16.1$ one month after, $\mathrm{P}<0.001$ ) were statistically significant with each other in all intervals time. Conclusion: the deployment of self-efficacy intervention can improve self-care ability in stroke patients despite the various problems that patients are involved.

\section{P125}

Effect of 5-HT1D Receptor Agonist on Hippocampal Long-Term Potentiation and Level of BDNF in Reinstated-Methamphetamine Rats

Reihaneh Sadeghian ${ }^{1}$ Siamak Shahidi', Alireza Komaki', Sara Soleimani Asl ${ }^{2}$

${ }^{1}$ Neurophysiology Research Center, Hamadan University of Medical Sciences, Hamadan, Iran

${ }^{2}$ Department of Anatomy, Faculty of Medicine, Hamadan University of Medical Sciences, Hamadan, Iran

The Neuroscience Journal of Shefaye Khatam, 2019; 7(3): P125

Introduction: Methamphetamine (METH) is a psychostimulant that the precise mechanisms of its effects remain unknown and current relapse treatments have low efficacy. However, brain-derived neurotrophic factor (BDNF) and neuronal plasticity are essential contributors, despite paradoxical reports and a lack of comprehensive studies. Serotonin is a key neuromodulator in this challenge. While there is a known distribution of 5-HT 1 receptor in the reward and memory areas, the physiological function of this receptor is currently unknown. Here, we evaluated effect of a $5-\mathrm{HT}_{1 \mathrm{D}}$ receptor agonist (PNU142633) on the electrophysiology recording and level of BDNF. Materials and Methods: Rats were implanted with a cannula into lateral ventricle. After recovery, animals treated with saline or METH $(5 \mathrm{mg} / \mathrm{kg})$ during acquisition of conditioned place preference (CPP). On day 13 extinction, METH groups divided into four groups. They received METH (0: saline, 1, or $2.5 \mathrm{mg} / \mathrm{kg}$; i.p.) + vehicle $(5 \mu \mathrm{l} / \mathrm{rat})$ or a priming dose of METH + PNU (2 $\mu \mathrm{g} / 5 \mu \mathrm{L}$; i.c.v.) and conditioning scores were calculated on reinstatement day. Finally, electrophysiology and western blotting tests were performed for measurements LTP components and expression of BDNF, respectively. Results: The results showed pre-treatment with PNU significantly reduced the reinstatement of priming METH seeking and level of BDNF expression was more than other groups. Furthermore, PS amplitude and EPSP slope in vehicle + priming METH rats were more than others. Also, PS amplitude in PNU+METH group was lower than priming METH group. Conclusions: These findings suggest that PNU can alter synaptic transmission and level of BDNF. Subsequently have therapeutic potential for preventing METH relapse. 
Recurrent Optic Neuritis and Neuromyelitis Optica Spectrum Disorders (NMOSD): a Case Report

\author{
Arian Saeidi ${ }^{1}$, Morteza Saeidi ${ }^{2}$ \\ ${ }^{1}$ Faculty of Medicine, Mashhad University of Medical Sciences, \\ Mashhad, Iran \\ ${ }^{2}$ Department of Neurology, Mashhad University of Medical \\ Sciences, Mashhad, Iran
}

The Neuroscience Journal of Shefaye Khatam, 2019; 7(3): P126

Neuromyelitis optica spectrum disorder (NMOSD) is severe rare chronic inflammatory disease of CNS that may be presents with blindness and paraparesis and is characterized by demyelinating lesions in spinal cord and optic nerve. In most patients we can find seropositivity for IgG_Abs against AQP4 that make us distinguish the disorder from MS. These autoantibodies target AQP4 on astrocytes, choroid plexus epithelial cells, ependymal cells, and Müller cells. NMOSD showed atrophy in brain regions related to clinical manifestations (visual cortex), but also at the level of areas with high expression of Aquaporin-4 antibodies (thalami and fourth ventricle). Certain clinical presentations which are Suggestive for NMOSD include: 1) Bilateral or unilateral optic neuritis which involves the optic chiasm or optic nerves (usually posterior parts), which can induce different varieties of visual loss or some degrees of visual field defects. 2) Complete or incomplete spinal cord syndrome especially presents with paresthesia, weakness of lower and upper limbs, urinary retention or incontinence, paroxysmal tonic spasm, sensory level and other clinical symptoms. 3) The involvement of Area postrema (up to one third of patients) which can induce severe and intractable hiccups, vomiting and nausea. Current strategies for NMO treatment are general immunosuppression (steroid therapy, cytotoxic agents), B_cell therapy (Rituximab) and plasma exchange. In this study we present some cases of recurrent optic neuritis, different degrees of paresthesia and also weakness of lower or upper limbs with relapsing manner (simultaneous or unsimultaneous), negative or positive NMO ab test which represent NMO spectrum disorders in seronegative or seropositive patterns.

\section{P127}

Nicorandil Effects on the Acute Brain Ischemia in MCAO-Induced Stroke Rat Model

\section{Khastkhodaei Ardekani ${ }^{1,2}$, A Safari ${ }^{3}$, Mohammad Reza Namavar ${ }^{2,4}$}

${ }^{1}$ Department of Anatomical Sciences, Faculty of Medicine, Shiraz University of Medical Sciences, Shiraz, Iran

${ }^{2}$ Histomorphometry and Stereology Research Centre, Shiraz University of Medical Sciences, Shiraz, Iran

${ }^{3}$ Stem Cells Technology Research Center, Shiraz University of Medical Sciences, Shiraz, Iran
${ }^{4}$ Clinical Neurology Research Center, Shiraz University of Medical Sciences, Shiraz, Iran

The Neuroscience Journal of Shefaye Khatam, 2019; 7(3): P127

Introduction: Stroke is the second leading cause of death worldwide. There is no effective and generally accepted treatment for the acute ischemic stroke except thrombolysis with recombinant tissue plasminogen activator that has many limitations. Nicorandil, a potassium ATP channel opener, decreases inflammation and prevents cell death. The aim of this study was to evaluate nicorandil effects on the acute brain ischemia. Materials and Methods: 28 male Sprague Dawley rats weighing 200-250g were randomly divided into four groups: the sham group that tolerated anesthesia and surgery without middle cerebral artery occlusion (MCAO) and drug or vehicle. The control, nicorandil 1 and $3 \mathrm{mg} / \mathrm{kg}$ groups underwent MCAO and received nicorandil solvent, nicorandil 1 and $3 \mathrm{mg} / \mathrm{kg}$ intraperitoneally, respectively. The behavioral recovery of rats was evaluated using Garcia score test. The ischemic size, the numerical density of neuron and dead neuron in cortex, striatum and hippocampus calculated by using stereological methods. Results: Behavioral tests showed that nicorandil improved neurological functions. Although nicorandil did not show significant changes in the brain total volume, it significantly decreased ischemic size that was more obvious in higher dose. Furthermore, the numerical density of neurons in treatment groups was significantly higher than the control group in higher dose and numerical density of dead neurons in treatment groups was significantly lower than the control group. Conclusion: Nicorandil improved not only the sensory and motor behaviors, but also it reduced ischemic size and these improvements were dose dependent. It also increased neuronal number in different parts of the brain and reduced number of dead neurons. Furthermore, the neurological improvements were correlated with histological findings. These results may show neuroprotective and anti-apoptotic effects of this drug, but it did not support its anti-inflammatory effect, at least in the acute phase of brain ischemia.

\section{P128}

The Effect of Dopaminergic Receptors Antagonist and CCPA (A1 Adenosine Receptor Agonist) or SCH58261 (A2a Adenosine Receptor Antagonist) on Morphine Withdrawal Syndrome

\section{Mojgan Rajabi ${ }^{1}$,Gisou Mohaddes ${ }^{1,2}$, Rasi $\mathbf{S}^{1}$}

${ }^{1}$ Neuroscience Research Center, Tabriz University of Medical Sciences, Tabriz, Iran

${ }^{2}$ Drug Applied Research Center, Tabriz University of Medical Sciences, Tabriz, Iran

The Neuroscience Journal of Shefaye Khatam, 2019; 7(3): P128

Introduction: Morphine withdrawal induces a state of 
neuronal hyperexcitability in the brain which has been linked to changes in a number of second messenger systems and neurotransmitters. In the present study, the effects of chlorpromazine (dopamine receptor antagonist) alone or in combination with CCPA (A1 adenosine receptor agonist) or SCH58261 (A2a Adenosine receptor antagonist) were examined on morphine withdrawal syndrome in mice. Materials and Methods: In this study, 80 NMRI male mice were allocated in 8 groups $(n=10)$ : saline, chlorpromazine $(0.25,0.5,1.0 \mathrm{mg} / \mathrm{kg})$, CCPA $(0.5 \mathrm{mg} / \mathrm{kg}), \mathrm{SCH} 58261$ $(0.1 \mathrm{mg} / \mathrm{kg})$, chlorpromazine $(0.5 \mathrm{mg} / \mathrm{kg})+\mathrm{CCPA}$, and chlorpromazine $(0.5 \mathrm{mg} / \mathrm{kg})+\mathrm{SCH}$. Animals were received different doses of morphine sulphate subcutaneously (s.c) and the withdrawal syndrome was evoked by naloxone injection (4 mg/kg i.p.). Animals were observed for $30 \mathrm{~min}$ for jumping behavior and diarrhea. CCPA (0.5 mg/kg i.p.) and SCH 58261 (1 $\mathrm{mg} / \mathrm{kg}$ i.p.) were given $10 \mathrm{~min}$, and chlorpromazine $30 \mathrm{~min}$ before naloxone. In combination therapy micetreated with chlorpromazine $0.5 \mathrm{mg} / \mathrm{kg}$ and CCPA or SCH58261 before naloxone. Results: Administration of the dopamine D1/D2 receptor antagonist or chlorpromazine $(0.25,0.5$ and $1 \mathrm{mg} / \mathrm{kg})$ significantly decreased jumping $(\mathrm{p}<0.05)$ and diarrhea $(\mathrm{p}<0.001)$. Both CCPA and SCH 58261 significantly reduced jumping $(p<0.001)$ and diarrhea $(p<0.001)$. Combination therapy of chlorpromazine and CCPA or SCH58261 also significantly decreased jumping and diarrhea $(\mathrm{p}<0.001)$. Conclusion: It is concluded that blockade of both dopamine receptors with chlorpromazine may suppress naloxone-induced jumping and diarrhea in morphinedependent mice. Effect of combination therapy of chlorpromazine and CCPA or SCH58261 on jumping and diarrhea were not additive.

\section{P129}

Protective Effect of Silymarin on LipopolysaccharideInduced on Learning and Memory Deficiency in Male Rat

\section{Fahimeh Nourbakhsh ${ }^{1}$, Samaneh Borooni ${ }^{2}$, Elaheh Tajbakhsh}

${ }^{1}$ Department of Pharmacodynamics and Toxicology, Faculty of Pharmacy, Mashhad University of Medical Sciences, Mashhad, Iran

${ }^{2}$ Department of Biology, Nourdanesh Institute of Higher Education, Esfahan, Iran

${ }^{3}$ Departments of Microbiology, Faculty of Basic Sciences, Shahrekord Branch, Islamic Azad University, Shahrekord, Iran

The Neuroscience Journal of Shefaye Khatam, 2019; 7(3): P129

Introduction: Lipopolysaccharide (LPS) or bacterial endotoxin triggers production of ROS and inflammatory cytokines. LPS is a major constituent of the outer membrane of gram-negative bacteria. Several studies have shown that, LPS induce oxidative stress in the hippocampus. Nowadays S. marianum have been shown to treat on the learning, memory deficiency and other organ toxicity. The purpose of this study is to verify the protective effect of S. marianum against LPS -induced memory deficiency in male rat. Materials and Methods: In this research, 40 male Wistar rats were divided into 4 groups ( $\mathrm{n}=9$ in each) in standard conditions: The animals were treated with $\mathrm{S}$. marianum for a 2 weeks before the biochemical tests, includes measurements of inflammatory and oxidative stress. Finally, initial (acquisition index) and step-through latencies (retention and recall index) were measured using passive avoidance test and alternation behavior percentage as an index of spatial memory was determined using $\mathrm{Y}$ maze. The level of malondialdehyde (MDA) in the homogenate hippocampus tissue of the animal's brains was measured. Results: The results of our investigation show that, LPS induced motor deficit as evidenced by histopathological damages and biochemical abnormalities $(\mathrm{P}<0.01)$. Data showed that MDA level was significantly increased in the hippocampus of LPS-treated rats but protective group's adjusted MDA level content $(\mathrm{P}<0.05)$. In addition, destructive effects of LPS on histopathological and biochemical parameters were improved. Western blot analysis show that S. marianum treatment inhibited apoptosis stimulated by LPS through decreasing the caspase 3, 8, 9 ratio level in the hippocampus tissue. Conclusion: The results of this research demonstrated that S. marianum could exert protective effects against toxic effects of LPS in rat hippocampus tissue. Antiinflammatory drug can play a protective role in attenuating of the hippocampus inflammation induced by LPS injection.

\section{P130}

Effects of Platelet-Rich Plasma (PRP) on the B92 Cell Primed with Killed Preparation of Escherichia Coli

\section{Mahtab Pourkamalzadeh, Seyyed Meysam Abtahi Froushani, Alireza Mahmoudian}

Department Microbiology, Faculty of Veterinary Medicine, Urmia University, Urmia, Iran

The Neuroscience Journal of Shefaye Khatam, 2019; 7(3): P130

Introduction: Some evidence indicated that PlateletRich Plasma (PRP) possesses anti-inflammatory and regenerative benefits. The purpose of the present study was to investigate the protective effects of PRP on the rat Glial/neuronal cell line B92 after exposure with killed preparation of Escherichia Coli. Materials and Methods: B92 cells were cultured in the absence or presence of E. coli (1:100 ratio) for $24 \mathrm{~h}$ in DMEM medium supplemented with FBS and different concentration of PRP $(0,5,10$ and $20 \%)$.Cellular cytotoxicity was measured by MTT assay. Results: 
Killed preparation of $E$. Coli reduced the proliferation of B92 cells in a concentration- dependent manner. PRP at 10 or $20 \%$ concentrations could reverse this reduction so that in the presence of $20 \%$ PRP, the proliferation of B92 cells were more profound that un-pulsed B92 with E.coli. Nevertheless, PRP at 5\% didn't show any significant effects on the proliferation of B92 cells primed with $E$. coli. Conclusion: In vitro data showed that PRP has protective effects against cell death induced by E. coli in Glial/neuronal cell line B92.

\section{P131}

Evaluation of Attention in ADHD Children in Comparison with Healthy Subjects Using EventRelated Potentials (ERPs)

\section{Farnoosh Atarodi, Majid Ghoshuni}

Department of Biomedical Engineering, Mashhad Branch, Islamic Azad University, Mashhad, Iran

The Neuroscience Journal of Shefaye Khatam, 2019; 7(3): P131

Introduction: Attention Deficit/Hyperactivity Disorder (ADHD) is one of the common mental disorders in childhood. ADHD is a disorder caused by brain malfunction, so electroencephalogram (EEG) analysis can be a useful ADHD diagnosis. On the other hand, using event related potentials (ERPs) for diagnosing ADHD has been one of the significant issues in previous studies. ERP components reflect neural activity pattern based on the perception of a given stimulus, so ERP can measure brain activity during intellectual processes in visual and auditory system. In this study, the correlation between ERP components and attention index has been investigated using Integrated Visual and Auditory Test (IVA). Materials and Methods: 9 healthy children ( 1 female) and 8 ADHD children (2 females) with an average age of 7.11 years participated in this study. EEG data were recorded by the Mitsar202 EEG system and the International Standard 20-10 was used for electrode placement. All of the 19 electrodes, including $\mathrm{Fz}, \mathrm{Cz}$, Pz, O1, O2, C3, C4, T3, T4, Fp1, Fp2, P3, P4, F3, F4, $\mathrm{F} 7, \mathrm{~F} 8, \mathrm{~T} 5, \mathrm{~T} 6$ have been used to record the brain signal and the mean of $\mathrm{A} 1$ and $\mathrm{A} 2$ for the left and right ears are used as reference. The subject has been asked to participate in the IVA test, and simultaneously ERP was recorded. Results: According to the results, there was a significant correlation $(\mathrm{r}=0.74$ and $\mathrm{P}=0.0005)$ between the delay time of the P300 component of the ERP signal in the P3 channel for auditory stimulation and the correct response of the subjects, Also, the correct response for auditory stimulus in the ADHD group was significantly lower than in the healthy children. Conclusion: The P300 component of the ERP signal can be considered as an appropriate tool for determining the level of auditory attention in ADHD children and distinguish them from healthy people.

\section{P132}

Combretastatin A-4 (CA-4) Induce P-53 Independent Apoptosis in Glioblastoma Cell Line (U-87MG)

Mostafa Karimi Roshan ${ }^{1,2}$, Sajjad SahabNegah ${ }^{4}$, Amir Reza Afshari', Mohammad Soukhtanloo'

${ }^{1}$ Department of Medical Biochemistry, Faculty of Medicine, Mashhad University of Medical Sciences, Mashhad, Iran

${ }^{2}$ Student Research Committee, Faculty of Medicine, Mashhad University of Medical Sciences, Mashhad, Iran

${ }^{3}$ Department of Pharmacology, Faculty of Medicine, Mashhad University of Medical Sciences, Mashhad, Iran

${ }^{4}$ Department of Neurosciences, Faculty of Medicine, Mashhad University of Medical Sciences, Mashhad, Iran

The Neuroscience Journal of Shefaye Khatam, 2019; 7(3): P132

Introduction: Glioblastoma is the most prevalent primary malignant brain tumor. Survival is mostly less than one year, and most patients die within two years. Standard therapy includes surgical resection and generally followed by radiotherapy and chemotherapy. CA4 as an antimitotic agent can strongly cause vascular shutdown and cell death in tumors by binding the colchicine binding site of tubulin to block microtubule assembly. The aim of this stud was to investigate the apoptotic and cytotoxic effect of CA4 in glioblastoma multiforme (U87) cell line. Materials and Methods: Cellular cytotoxicity was measured by rezasurin assay and intracellular active oxygen species were measured using DCFDA (2 'and 7'-dichloro fluorescein diacetate) assay kit. In addition, apoptotic cells were detected with an Annexin V-FITC early apoptosis staining and cell cycle assay was investigated by propidium iodide (PI). Furthermore, Reatime PCR used to detect the alteration in gene expression of Apoptotic genes. Results: CA4 reduced the proliferation of U87 cells in a concentrationdependent manner. IC50 was 14 and $12.5 \mu \mathrm{g} / \mathrm{mL}$ after 24 and 48 hours, respectively. No significant increase observed in reactive oxygen spices level. Cell cycle distribution analysis showed significant increase in cell population in sub G1 area in concentration-dependent manner. Moreover, Anexin/PI analysis showed an increase in apoptotic cells after treatment by CA4. Significant alteration is not observed in gene expression of Bax, Bcl-2, and P-53. Conclusion: The study show that CA-4 can induce apoptosis in glioblastoma cells which seems it is p53-independent.

\section{P133}

The Effect of Ethyl Acetate Extract of Oleo-GumResin of Ferula Assa-Foetida L. on Painful Diabetic Neuropathy in Adult Male Rats

Samad Nazemi ${ }^{1}$, Hamid Rudsarabi ${ }^{2}$, Bahareh Amin ${ }^{3}$, Hasan Azhdari Zarmehri ${ }^{4}$ 
'Department of Physiology and Pharmacology, Faculty of Medicine, Sabzevar University of medical Sciences, Sabzevar, Iran

${ }^{2}$ Student Research Committee, Sabzevar University of Medical Sciences, Sabzevar, Iran

${ }^{3}$ Cellular and Molecular Research Center, Faculty of Medicine, Sabzevar University of Medical Sciences, Sabzevar, Iran

${ }^{4}$ Department of Basic Sciences and Neurosciences Research Center, Torbat Heydariyeh University of Medical Sciences, Torbat Heydariyeh, Iran

The Neuroscience Journal of Shefaye Khatam, 2019; 7(3): P133

Introduction: Painful diabetic neuropathy is a wellknown and often progressive complication in both types of diabetes which does not respond well to the common pain medications. Using medicinal herbs can help to find new pain medications with more efficacy and less side effects. This study aimed to evaluate the effect of ethyl acetate extract of Asafoetida on painful diabetic neuropathy induced by streptozotocin (STZ) in adult male rats. Materials and Methods: In this experiment,40 male Wistar rats (230-280 g) were used. Diabetes was induced by intraperitoneal (ip) injection of single dose of STZ $(60 \mathrm{mg} / \mathrm{kg})$. Animals were randomly divided into five groups including: control, diabetic and 3diabetic groups were treated with the Asafoetida extract at dose of $25,50,100 \mathrm{mg} / \mathrm{kg}$ for 25 days. To evaluate the neuropathic changes, Tail-flick and vonFrey tests were performed on post diabetic days (PDD) 0, 7, 14, 21 and 28, $(\mathrm{P}<0.05)$. Results: Behavioral results showed that, diabetic neuropathy was established in PDD 21. Asafoetida extract with the dose of $50 \mathrm{mg} /$ $\mathrm{kg}(P<0.05)$ and $100 \mathrm{mg} / \mathrm{kg}(P<0.01)$ prevented the induction of thermal hyperalgesia, whereas this response was observed only for the dose of $100 \mathrm{mg} / \mathrm{kg}$ in the case of mechanical allodynia $(P<0.05)$. In addition, blood glucose concentration in the extract treated groups was decreased in a dose-dependent manner $(P<0.05)$. Conclusion: Ethyl acetate extract of asafoetida can prevent the development of painful diabetic neuropathy. Further studies are required to identify the bioactive components present in this extract and their mechanisms of action in reducing diabetic neuropathic pain.

\section{P134}

Alterations of Neuro-Immune Cells Density and ProInflammatory Cytokines in Response to Thimerosal in Prefrontal Lobe of Male Rat

Kobra Afsordeh ${ }^{1}$, Yousef Sadeghi', Abdollah Amini $^{1}$, Zahra Namvarpour ${ }^{2}$, Mohammad-Amin Abdollahifar', Abass Aliaghaei ', Hojjat-Allah Abbaszadeh ${ }^{I}$

${ }^{1}$ Department of Biology and Anatomy, Faculty of Medicine, Shahid Beheshti University of Medical Sciences, Tehran, Iran ${ }^{2}$ Institute for Cognitive Science Studies (ICSS), Tehran, Iran
The Neuroscience Journal of Shefaye Khatam, 2019; 7(3): P134

Introduction: There is much evidence that indicates the effect of heavy metals on neuro-immune cells, which lead to neurogenic inflammatory responses. In this study, the immune cells (mast cells (MCs), and microglia), and the pro neuroinflammation cytokines (Interleukin-1b (IL-1b) and Tumor Necrosis Factor- $\alpha$ (TNF- $\alpha)$ ) were assessed in the prefrontal lobe of rat brain exposed to Thimerosal $(49.55 \%$ ethyl-mercury by weight) in different timeframes. Materials and Methods: 108 neonatal Wistar rats were divided into three groups with three subgroups : 1) The experimental groups taked a single dose of Thimerosal $(300 \mu \mathrm{g} / \mathrm{kg})$ on postnatal days 7, 9, 11and 15. 2) The Vehicle groups received PBS (Ph. =7.4) on the same order and 3) The control groups received nothing. The samples of the prefrontal cortex were collected and prepared for soteriological, Immunohistochemistry and molecular studies at different timeframe including $12 \mathrm{~h}, 48 \mathrm{~h}$ (as acute phases), and 8 days (as subchronic phase) after the last injection, respectively. Results: The average density of microglial and mast cells was significantly increased in experimental groups. This increase was more dominant in $48 \mathrm{~h}$. In 8 days after injection, there was a significant decrease in density of mast cells in compared with $12 \mathrm{~h}$ and $48 \mathrm{~h}$. Alterations of pro inflammatory cytokines were significant in all timeframes. This increase was more dominant in $48 \mathrm{~h}$ after last injection and also, there was a significant decrease in both neuro- inflammatory cytokines after 8 days. Conclusion: Ethyl-mercury led to abnormal neurogenic inflammatory reactions and that alterations in neuro-immune cells can be remained for longer period in brain than blood.

\section{P135}

Feeding Vitamin C During Neonatal and Juvenile Growth Improve Learning and Memory of Rats

\section{Masoumeh Gholami ${ }^{1,2}$, Mahmoud Hosseini ${ }^{3}$, Farimah Beheshti $^{1,2}$}

${ }^{1}$ Neuroscience Research Center, Torbat Heydariyeh University of Medical Sciences, Torbat Heydariyeh, Iran

${ }^{2}$ Department of Physiology, Faculty of Paramedical Sciences, Torbat Heydariyeh University of Medical Sciences, Torbat Heydariyeh, Iran

${ }^{3}$ Department of Neurocognitive Sciences, Psychiatry and Behavioral Sciences Research Center, Mashhad University of Medical Sciences, Mashhad, Iran

The Neuroscience Journal of Shefaye Khatam, 2019; 7(3): P135

Introduction: We investigated the effects of feeding vitamin $\mathrm{C}$ (Vit $\mathrm{C}$ ) during neonatal and juvenile growth on learning and memory of rats. Rats after delivery were randomly divided into four groups and treated. Materials and Methods: Rats after delivery were randomly divided into four groups and treated. Group 1, control group, 
received normal drinking water. Groups 2-4 received Vit C 10, 100, and $500 \mathrm{mg} / \mathrm{kg}$, respectively, from the first day. After 8 weeks, 10 male offspring of each group were randomly selected and tested in the Morris water maze (MWM) and passive avoidance (PA) tests. Finally, the brains were removed for biochemical measurement. Results: In MWM, 10-500 mg/kg Vit $\mathrm{C}$ reduced the latency and traveled distance and increased time spent in the target quadrant. In PA, 10 and $100 \mathrm{mg} /$ $\mathrm{kg}$ of Vit $\mathrm{C}$ increased the latency; $10-500 \mathrm{mg} / \mathrm{kg}$ of Vit $\mathrm{C}$ decreased the malondialdehyde (MDA) in the brain tissues and increased thiol and catalase (CAT) activity compared to the control group. Conclusion: We showed that feeding rats Vit $\mathrm{C}$ during neonatal and juvenile growth has positive effects on learning and memory.

\section{P136}

Comparison the Effect of Pentylentetrazole and Kainic Acid Convulsive Potential

Giti Nasudi', Mahmoud Elahdadi Salmani', Narges Hoseinmardi ${ }^{2}$, Taghi Lashkarbolouki ${ }^{1}$, Iran Goudarzi ${ }^{1}$

'Department of Animal Biology, Faculty of Biology, Damghan University, Damghan, Iran

${ }^{2}$ Department of Physiology, Faculty of Medicine, Shahid Beheshti University of Medical Sciences, Tehran, Iran

The Neuroscience Journal of Shefaye Khatam, 2019; 7(3): P136

Introdution: The brain is affected by severe seizure activities which can damage the brain and change its structure and function. Seizure models affect the brain differently which may have a negative impact on the outcome of the experiments. The greater the severity of the seizure, the greater the damage to the neuronal network. Therefore, in this study, the convulsive effects of kainic acid and pentyltentetrazole will be compared. Materials and Methods: In this experimental study, 24 adult male Wistar rats were allocated into two groups. In the first group, seizure was induced by a single intravenous injection petylentetrazole $(30 \mathrm{mg} / \mathrm{Kg}$ i.v). The second group, a single injection of the kainic acid inside the hippocampus $(4 \mu \mathrm{g} / 5 \mu \mathrm{l})$ provoked the seizure. The behavioral profile was characterized by stages defined as scores: (0) short swim, (1) increased swimming activity and high frequency of opercular movement, (2) erratic movements, (3) circular movements, (4) clonic seizurelike behavior, (5) fall to the bottom of the tank and tonic seizure-like behavior, (6) death. Results: The results of this study showed, the latency 1,2 for the onset of seizure induced by pentyltentetrazole decreased significantly compared with the kainic acid group $(\mathrm{P}<0.01)$. Duration of seizure $(\mathrm{P}<0.01)$, the percentage of tonic - clonic seizures and mortality rate in the pentyltentetrazole group increased significantly. Conclusion: Based on the results of this study, the pentylentetrazole seizure activity are stronger and faster than the kainic acid and may have a stronger impact on the neural functions.

\section{P137}

Evaluation of the Prevalence of Multiple Sclerosis and its Important Risk Factors in Iran from 2005 through 2018: "a Systematic Review"

\section{Parisa Mamivand ${ }^{1}$, Arman Jafari ${ }^{2}$}

${ }^{1}$ Research Committee, kashan University of Medical Sciences, Kashan, Iran

${ }^{2}$ Shoushtar Faculty of Medical Sciences, Shoushtar, Iran

The Neuroscience Journal of Shefaye Khatam, 2019; 7(3): P137

Introduction: Multiple sclerosis is a demyelinating disease which is the most common immune-mediated disorder. The epidemiology of MS is vary from region to region in Iran. but several studies show that this disease has been increasing all over the country. In this study the aim was to provide a comprehensive review, regarding the prevalence of MS across Iran. Materials and Methods: The present study uses systematic searches of articles in the internal database (SID, Iran Medex and Magiran) and external authoritative databases (Google Scholar, Pub Med, Science Direct, MEDLINE, CINAHL and EMBASE) using keywords including "prevalence"," Iranian"," Multiple Sclerosis "and" Incidence "have been gathered since2005 to 2018. In this study 62 articles were collected. Finally, 16 studies were used. Results: The prevalence of MS in Iran according to the articles ranged from 14 in 100,000 to 116 in 100,000 people. The highest prevalence was in Tehran in 2015 (115.94 per $100,000)$ and the lowest prevalence in Sistan-Baluchistan (13.9 per 100,000) .The prevalence of MS in Iran has increased in recent decades, which can be attributed to air pollution. Also among Iranian relatives, people with Lor's parents are more likely to have this disease compare to other ethnicties. In addition, the heterogeneity of the ethnicity of the parents, increases the risk of MS .The prevalence rate in women is about three times as high as in men in the last decade. The high prevalence in women may have association with gender issues such as the onset of menstruation, the age of the first pregnancy, and use of OCPs. Another factor of this disease is vitamin $\mathrm{D}$ deficiency in women due to lifestyle in Iran and less exposure to sunlight. Conclusion: Due to the relatively high prevalence of this disease, it is important to do the necessary actions to prevent the disease. Also, people with vitamin D deficiency, which is one of the predisposing factors, can be cured. According to the findings, inheritance is one of the factors of the disease that health care administrators should identify susceptible families, and advise them to take preventive and therapeutic measures. Also, due to the high prevalence of women, it is necessary for authorities to pay more attention to women.

\section{P138}

The Effects of Nickel Oxide Nanoparticles on Tau Protein and Neuron-Like Cells: Biothermodynamics 
and Molecular Studies

Mozhdeh Hajimohammadjafartehrani, ${ }^{1}$ Sara Hajihosseinali $^{2}$ Abolfazl Dehkohneh ${ }^{3}$, Pegah Ghoraeian $^{2,4}$, Mahsaale-Ebrahim ${ }^{1}$, Keivan Akhtari ${ }^{3,4}$, Koorosh Shahpasand ${ }^{5}$, Ali Akbarsaboury, Farnoosh Attar $^{7}$ Mojtaba Falahati ${ }^{8}$

${ }^{1}$ Department of Cellular and Molecular Biology, Faculty of Advanced Science and Technology, Islamic Azad University, Tehran, Iran

${ }^{2}$ Department of Genetics, Faculty of Advanced Science and Technology, Islamic Azad University, Tehran, Iran

${ }^{3}$ Department of Biotechnology, Faculty of Advanced Science and Technology, Islamic Azad University, Tehran, Iran

${ }^{4}$ Department of Physics, University of Kurdistan, Sanandaj, Iran

${ }^{5}$ Cell Science Research Center, Royan Institute for Stem Cell Biology and Technology (ACECR), Department of Brain and Cognitive Sciences, Tehran, Iran

'Institute of Biochemistry and Biophysics, University of Tehran, Tehran, Iran

${ }^{7}$ Standard Research Institute (SRI), Department of Biology, Faculty of Food Industry and Agriculture, Karaj, Iran

${ }^{8}$ Department of Nanotechnology, Faculty of Advanced Science and Technology, Islamic Azad University, Tehran, Iran

The Neuroscience Journal of Shefaye Khatam, 2019; 7(3): P138

Herein, the thermodynamic parameters of tau upon interaction with NiO NPs were determined by fluorescence spectroscopy. Also, molecular docking studies were run to explore the binding affinities of $\mathrm{NiO}$ NPs clusters with different sizes of $30 \AA$ and $50 \AA$ toward tau. Also, cytotoxic activity of $\mathrm{NiO} \mathrm{NPs}$ against SH-SY5Y was determined by MTT, LDH, caspase-9/3 activity, and expression of apoptotic Bax and Bcl-2 genes assays. DLS study showed that $\mathrm{NiO}$ solution had a good colloidal stability. Fluorescence study revealed that $\mathrm{KSV}$ values were $2.95 \pm 0.35 \times 104,3.31 \pm 0.59 \times 104$ and $3.92 \pm 0.65 \times 104$ at $298 \mathrm{~K}, 310 \mathrm{~K}$ and $315 \mathrm{~K}$, respectively. Also, $\Delta \mathrm{G}^{\circ}(\mathrm{kJ} / \mathrm{mol}), \Delta \mathrm{H}^{\circ}(\mathrm{kJ} / \mathrm{mol})$ and $\mathrm{T} \Delta \mathrm{S}^{\circ}(\mathrm{kJ} / \mathrm{mol})$ values were $-13.27 \pm 1.57,1.98 \pm 0.14$, $15.25 \pm 2.01$, respectively at $298 \mathrm{~K}$. Theoretical studies depicted that affinity of 5O3T segment toward NiO NP (30 $\AA)$ is higher than NiO NP (50 $\AA$ ) and the proportion of Lys residues are higher in the docked pose of NiO NP $(30 \AA) / 5 \mathrm{O} 3 \mathrm{~T}$ complex than NP $(50 \AA) / 5 \mathrm{O} 3 \mathrm{~T}$ complex. Moreover, NiO NPs demonstrated a significant increase in the mortality of SH-SY 5Y cells in an apoptotic manner. This study determined that NiO NPs may mediate the formation of electrostatic interactions with tau and induction of undesired harmful effects on neurons.

\section{P139}

C. Aurantium Peel and Seed Extracts Exhibit Neuroprotective Effect Against Glutamate-Induced Toxicity in PC12 Neuronal Cells

Arezoo Rajabian', Azar Hosseini', Hamid Reza

\section{Sadeghnia}

${ }^{1}$ Pharmacological Research Center of Medicinal Plants, Faculty of Medicine, Mashhad University of Medical Sciences, Mashhad, Iran

${ }^{2}$ Department of Pharmacology, Faculty of Medicine, Mashhad University of Medical Sciences, Mashhad, Iran

The Neuroscience Journal of Shefaye Khatam, 2019; 7(3): P139

Introduction: Neurodegenerative diseases have been associated with glutamatergic dysfunction. Recent studies have demonstrated several pharmacological effects including anti-inflammatory, neuroprotective, and anticonvulsant properties of Citrus aurantium. In this study, we aimed to investigate the effects of $C$. aurantium peel and seed extracts against glutamateinduced oxidative damage and apoptosis. Materials and Methods: The cultured PC12 cells were pretreated $(2 \mathrm{hr})$ with varying concentrations of the extract (6 to $200 \mu \mathrm{g} / \mathrm{ml})$, followed by exposure to glutamate $(8 \mathrm{mM})$ for $24 \mathrm{hr}$. The cells viability was then measured using the MTT assay. Intracellular reactive oxygen species (ROS) and lipid peroxidation were evaluated using flurimetric assays as well as apoptosis was detected by PI staining. Results: Glutamate cytotoxicity in PC12 cells was accompanied by an increment of MDA content, ROS generation, and apoptotic induction ( $\mathrm{p}<$ 0.01 ). However, pretreatment with peel and seed extracts of $C$. aurantium significantly increased cell viability following glutamate insults at concentrations higher than $12 \mu \mathrm{g} / \mathrm{ml}(\mathrm{p}<0.01)$. The cytoprotective potential of $C$. aurantium was also accompanied by decreased ROS accumulation, malondialdehyde level $(\mathrm{p}<0.01$ 0.001 ), and apoptotic cells following glutamate insults. The results suggest that $C$. aurantium protects against glutamate-induced PC12 cells injury by decreasing oxidative stress and subsequently inhibiting apoptosis. Conclusion: $C$. aurantium peel and seed extracts could inhibit the glutamate-induced neurotoxicity, and these extracts have the potential to be used as a new therapeutic agent for neurodegenerative disorders. The exact mechanisms and the active compounds that are responsible for the anticonvulsive effect need to be clarified in future studies.

P140

Preparation and Evaluation of Nanoliposomal Formulation of Glatiramer Acetate for Treatment of Multiple Sclerosis

\section{Niloufar Rahiman, Mahmoud Reza Jafari}

Department of Pharmaceutical Nanotechnology, Faculty of Pharmacy, Mashhad University of Medical Sciences, Mashhad, Iran

The Neuroscience Journal of Shefaye Khatam, 2019; 7(3): P140

Introduction: Multiple sclerosis (MS) is an 
autoimmune disease of the CNS in which cellular immunity and inflammatory cytokines lead to loss of myelin. Glatiramer acetate is a random copolymer of 4 amino acids, approved for treatment of MS. Due to site and glomerular elimination of glatiramer acetate after subcutaneous injection, we determined to design its liposomal form in which some properties of drug such as size are modulated to prevent its hydrolysis and also leading to stimulation of humoral immunity. Materials and Methods: The liposomes were produced through film method with various percentage of different phopholipids. Then size, zeta potential, percentage of encapsulation were evaluated. Cellular cytotoxicity will measured by MTT assay. The liposomal formulation of Glatiramer Acetate will be injected subcutaneously to $\mathrm{C} 57 \mathrm{BL} / 6$ mice to evaluate immunological response and titration of cytokines. The effectiveness of this new formulation then will be compared to the conventional form of drug by inducing experimental autoimmune encephalomyelitis (EAE) in the aforementioned mice. Results: Three best formulation of liposomal Glatiramer Acetate (Dipalmitoylphosphatidylcholine D i pa $1 \mathrm{~m}$ it oy $1 \mathrm{phos} \mathrm{phat}$ id y lgly cerole, D i st e a r o y $1 \mathrm{phos}$ h h a t d y l c holi n e, Distearoylphosphatidylglycerol, Dimyristoyl phosphatidylcholine, Dimyristoylphosphatidylglycerol and Cholestrole) had the $Z$ Average of about 300nm, PDI of about 0.1 and zeta potential of about 25 . These three formulations reduced the proliferation in macrophage and fibroblast cells (J774 and NIH) in a concentration- dependent manner. Conclusion: It seems that liposomal form of glatiramer acetate has the potential to use as novel form of drug for treatment of MS with more efficacy and more duration of action than the conventional form of Glatiramer Acetate that will enhance compliance of patients.

\section{P141}

\section{Quercetin-Loade Chitosan-Alginate-STPP Nanoparticles Ameliorate Memory Deficits and Reduce Glial Activation in Pentylenetetrazol- Induced Kindling Model of Epilepsy}

Atefeh Akbari', Mona Hashemian', Seyed Raheleh Ahmadian', Maryam Ghasemi-Kasman ${ }^{2,3}$

\begin{abstract}
'Student Research Committee, Babol University of Medical Sciences, Babol, Iran

${ }^{2}$ Cellular and Molecular Biology Research Center, Health Research Institute, Babol University of Medical Sciences, Babol, Iran

${ }^{3}$ Neuroscience Research Center, Health Research Institute, Babol University of Medical Sciences, Babol, Iran
\end{abstract}

The Neuroscience Journal of Shefaye Khatam, 2019; 7(3): P141

Introduction: Despite several beneficial effects of quercetin, its medical application has been hampered due to low water solubility. To improve the aqueous solubility of quercetin, it has been loaded on chitosan (CS)-alginate (ALG) - sodium tripolyphosphate (STPP) nanoparticles (NPs). Then, the effect of quercetin NPs on memory improvement and glial activation was investigated in pentylenetetrazol (PTZ)-induced kindling model. Materials and Methods: Male NMRI mice have received the daily injection of quercetin NPs at dose of 25 or $50 \mathrm{mg} / \mathrm{kg}$. All interventions were injected intraperitoneally (i.p), 10 days before PTZ administration and the injections were continued until $1 \mathrm{~h}$ before each PTZ injection. Animals have received 12 injections of $\mathrm{PTZ}$ and then, brain tissues homogenates were provided for assessment of oxidative stress markers, including thiobarbituric acid-reactive substances (TBARS) indicator of lipid peroxidation (LPO) and ferric-reducing ability of plasma (FRAP) indicator of total anti-oxidant capacity. Also, brain tissues were removed for histological evaluation. Nissl staining was used to determine the level of cell death in hippocampus and immunostaining method was performed against NeuN and GFAP/Iba1 for assessment of neuronal density and glial activation respectively. Results: Behavioral results showed that quercetin NPs exhibit anticonvulsant activity and prevent cognitive impairment in fully kindled animals. The level of cell death and glial activation reduced in animals which have received quercetin NPs compared to those received free quercetin. The level of TBARS decreased and FRAP increased in animals which have received quercetin NPs. Conclusion: To conclude, these findings suggest that quercetin NPs effectively ameliorate memory impairment and attenuate the level of activated glial cells in a mice model of chronic epilepsy.

\section{P142}

The Effects of Curcuma Longa and its Major Component, Curcumin on Nervous System Diseases: Anti-Inflammatory Effects

\section{Pouya Farahmand Niya ${ }^{1}$, Hamed Tahmasbi ${ }^{2}$, Farimah Beheshti ${ }^{3}$}

${ }^{1}$ Student Research Committee, Torbat Heydariyeh University of Medical Sciences, Torbat Heydariyeh, Iran

${ }^{2}$ Neuroscience Research Center, Department of Medical Basic Sciences, Torbat Heydariyeh University of Medical Sciences, Torbat Heydariyeh, Iran

${ }^{3}$ Psychiatry and Behavioral Sciences Research Center, Mashhad University of Medical Sciences, Mashhad, Iran

The Neuroscience Journal of Shefaye Khatam, 2019; 7(3): P142

Introduction: Curcuma longa is extensively used as a spice, food preservative and coloring material in Asia. it has been used in traditional medicine as a household remedy for various diseases. Curcumin also known as an antioxidant, anti-inflammatory and has a potential role in the prevention and treatment of nervous system diseases. Materials and Methods: We searched the 
literature available in Scopus, PubMed and Science Direct databases for English articles published until 1990. For this purpose we used 5 keywords for curcumin and Curcuma longa, 10 for animal models and 7 for nervous system disorders. Results: This review article summarized a variety of in vitro and in vivo studies in order to find out the effects of Curcuma longa extract and curcumin on neuroinflammatory disorders. The results of several important studies have shown that curcumin has more beneficial effects in different nervous system diseases including Alzheimer's disease (AD), cerebral edema (caused by traumatic brain injury) dementia, anxiety, depression, epilepsy and migraines than Curcuma longa. They can decrease IL (interleukin)-6, TNF (Tumor necrosis factor)- $\alpha$ and IL-1 $\beta$ and increase IL-10. Conclusion: The present review is an effort to provide a detailed scientific literature survey about anti-inflammatory effect of Curcuma longa extract and curcumin. Results showed that curcumin which founds in Curcuma longa can be considered as promising agents in the treatment of neuroinflammatory disorders.

\section{P143}

Effect of Sex Difference in HPA Axis Response to Involuntary Moderate Exercise in PTSD Rats

Sakineh Shafia ${ }^{1}$ Ali Rashidy-Pour ${ }^{2}$, Abbas Ali Vafaei $^{2}$, Simin Ehsani

${ }^{1}$ Department of Immunology Physiology, Faculty of Medicine, Mazandaran University of Medical Sciences, Sari, Iran

${ }^{2}$ Department of Physiology, Faculty of Medicine, Semnan University of Medical Sciences, Semnan, Iran

The Neuroscience Journal of Shefaye Khatam, 2019; 7(3): P143

Introduction: Post-traumatic stress disorder (PTSD) is a condition that develops after an individual has experienced a major trauma and have special symptoms for example: inability to extinguish fear memories, increase behavioral anxiety, disturbance in HPA axis and BDNF decrease. Moderate response rates and side effects to common treatment indicate an urgent need for the development of new treatment. Physical activity is known to improve symptoms of certain neuropsychiatric disorders. Interaction between stress, fear and sex are related to activity in HPA axis, and Stress response is moderated by these factors. This regulation is damaged in PTSD. Response to stress is not to same in male and female. In female this axis have faster responses and release more stress hormones than male. Estrogen and testosterone control HPA axis response. Testosterone inhibit HPA axis response and estrogen increasing activity in this axis. The present study investigated the effects of moderate treadmill exercise on HPA axis dysfunction in PTSD rats. Materials and Methods: We used to single prolonged stress (SPS) model as animal model for PTSD. The rats divided two groups; SPS and Sham group. Rats in SPS group were exposed to (restraint for $2 \mathrm{~h}$, forced swimming for $20 \mathrm{~min}$ and ether anesthesia) and were then kept undisturbed for 14 days. After that, SPS rats were subjected to moderate treadmill running (4 weeks, 5 days per week) and the followed by HPA axis activity in male and female PTSD rats by DST. Results: SPS male and female rats exhibited enhanced negative feedback on the HPA axis following a dexamethasone suppression test. Conclusion:Moderate treadmill exercise alleviated the SPS-induced alterations in HPA axis inhibition and there was no significant difference between male and female rats response to exercise.

\section{P144}

The Role of Mineral Serum Level in Risk Factor of Stroke. Babol, North of Iran

Alijan Ahmadi Ahangar', Soraya Khafri ${ }^{2}$, Payam Saadat ${ }^{1}$, Shayan Alijanpour ${ }^{3}$

${ }^{1}$ Mobility Impairment Research Center, Babol University of Medical Sciences, Babol, Iran

${ }^{2}$ Department of Biostatistics and Epidemiology, Babol University of Medical Sciences Babol, Iran

${ }^{3}$ Critical Care Nursing, Student Research Committee, Isfahan University of Medical Sciences. Isfahan, Iran

The Neuroscience Journal of Shefaye Khatam, 2019; 7(3): P144

Introduction: The relationship between stroke and mineral status is inconsistent. With regard to doubt in the role of mineral, this study was conducted. Materials and Methods: This cross-sectional study on 216 stroke patients who referred to the Ayatollah Rouhani Hospital of Babol over one-year period were conducted. Serum level of calcium, magnesium, Vitamin D (VD) were measured. Chi-square or Fisher exact test in spss 23 used. Results: Difference of hemorrhagic subtype was statistically significant in hypertensive patients (20(87\%) ICH vs. 1(12.5\%) SAH, p=0.001). In thrombotic patients, 98 cases $(89 \%)$ low VD vs. 12 cases $(10.9 \%)$ normal VD, $(p=0.001)$ were seen. Also, low VD was more seen significantly in DM patients $72(90 \%)$ in compare to non-DM patients $96(72.7 \%),(\mathrm{p}=0.003)$. Of 120 cases $(56.3 \%)$ who had HTN, 103 cases $(85.8 \%)$ low VD vs. 17 cases $(14.2 \%)$ normal VD, $(\mathrm{P}=0.01)$, 68 cases $(57.1 \%)$ hypo calcemic vs. 45 cases $(37.8 \%)$ normal $\mathrm{Ca}$ serum level, $(\mathrm{P}<0.0001)$ and 57 cases (49.1\%) hypomagnesemia vs. 59 cases $(50.9 \%)$ normal magnesium $(p=0.04)$ were seen. Difference in sex was statistically significant with hypomagnesemia (49 cases (53.8\%) male vs. 38 cases $(33.9 \%)$ female, $p=0.007)$. Hypomagnesemia were seen in 87 cases $(40.8 \%)$ that 49 cases of this were hypo-calcemic $(56.3 \%),(p=0.03)$. Differences in magnesium with VD was statistically significant (170 cases (79.4\%) low VD vs. 44 cases (20.6\%) normal, $p=0.007)$. Conclusion: Low VD in thrombotic and diabetic patients, low $\mathrm{Ca}$ and VD in 
hypertensive patients and hypocalcemia were more seen in hypermagnesemic patients. Preventive policies such as screening of mineral serum level are suggested.

\section{P145}

What Is the Combined Effect of Intense Intermittent Exercise and Ginkgo Biloba Plant on the Brain Neurotrophic Factors Levels, and Learning and Memory in Young Rats?

\section{Masoumeh Sadeghinejad ${ }^{1}, Z^{2}$ hra Soltani ${ }^{2}$ \\ 'Department of Sport Sciences, University of Birjand, Birjand, Iran \\ ${ }^{2}$ Endocrinology and Metabolism Research Center, Institute of Basic and Clinical Physiology Sciences, Kerman University of Medical Sciences, Kerman, Iran}

The Neuroscience Journal of Shefaye Khatam, 2019; 7(3): P145

Introduction: The present study was conducted to investigate the effect of intense intermittent exercise and Ginkgo biloba on the hippocampal levels of brainderived neurotrophic factor (BDNF) and neurotrophin-4 (NT-4) and also memory and learning in young rats. Materials and Methods: Forty- two eight-week-old rats were randomly divided into six groups including control, low dose of Ginkgo biloba (65 mg/kg), high dose of Ginkgo biloba (100 mg/kg), exercise, exercise + low dose of Ginkgo biloba, exercise + high dose of Ginkgo biloba. The exercise protocol or Ginkgo biloba administration was six days a week for six weeks. The hippocampal levels of BDNF and NT-4 were measured by ELISA method, and learning and memory were evaluated by Morris water maze test in all groups. Data were analyzed using SPSS software. Results: Increase in hippocampal levels of BDNF and NT-4 appeared following exercise $(p<0.01)$. The levels do not change following exercise + Ginkgo biloba administration. However, the NT-4 level decreased in the high dose of Ginkgo biloba group $(p<0.01)$. Disorder in learning and memory was indicated following the use of low dose of Ginkgo biloba or exercise + low dose Ginkgo biloba administration $(p<0.001)$. Learning elevated in the exercise group $(p<0.05)$. Conclusion: Exercise in young rats may increase brain neurotrophin levels and lead to improved learning. The preventative or protective role of Ginkgo biloba against some diseases has been suggested, but its consumption in young athletes is recommended with caution.

\section{P146}

The Effect of Air-Pollution on MS Patient's Relapses

\section{Amirhossein Ghaedaminiasadabadi}

Student Research Committee, Shahrekord University of Medical Sciences, Shahrekord, Iran

The Neuroscience Journal of Shefaye Khatam, 2019; 7(3): P146
Introduction: Multiple sclerosis is an autoimmune chronic inflammatory demyelinating disease of the central nervous system. Its prevalence and incidence rate has increased globally, consequently in Iran. Although some studies implicated the association between MS and concentration of vitamin D, sunlight, lifestyle, smoking, genetic predisposition (HLA allele), infections(EBV), climate and seasonal changes and number of siblings, triggers of MS relapsing are still essentially unknown. In this study the effect of air-pollution on MS relapsing $\mathrm{s}$ investigated. Materials and Methods: (air-pollution, Multiple Sclerosis and relapsing) were searched as keywords in (Pub med, Google scholar, Scopus) databases. Results: 100 articles were found at last 12 of them were selected (2011-2018). These studies reveals that the air-pollution ingredients such as $\mathrm{Fe}, \mathrm{Cu}, \mathrm{Cd}$, $\mathrm{PM} 10, \mathrm{SO} 2, \mathrm{NOX}, \mathrm{Pb}, \mathrm{Ni}, \mathrm{Mn}, \mathrm{Zn}$ and $\mathrm{O} 3$ especially in industrial areas are associated with CNS oxidative stress, neuro-inflammation, neuronal damaging, endothelial cell damage in the cerebral vasculature and blood brain barrier. In addition, they can have various effects by their interactions in multi-pollutant models, for instance different effects by season changing. Conclusion: The exposure to the air-pollution in particular PM10 could have direct relation with MS relapsing. Carrying out such researches in this field to making suitable models describing prognosis of air-pollution effect on MS can even reach to triggers of MS relapsing and decrease its complications.

\section{P147}

Effects of Vitamin E Supplementation on Amyotrophic Lateral Sclerosis

Ronak Jalali ${ }^{1}$, Marzieh Akbarzadeh ${ }^{2}$, Mohammad Jalali $^{2}$

${ }^{1}$ Faculty of Gorgan Medical, Gorgan University of Medical Sciences, Gorgan, Iran

${ }^{2}$ Faculty of Nutrition and Food Sciences, Shiraz University of Medical Sciences, Shiraz, Iran

The Neuroscience Journal of Shefaye Khatam, 2019; 7(3): P147

Amyotrophic lateral sclerosis (ALS) is a progressive neurodegenerative disease. Oxidative stress, apoptosis and neuro-inflammation are involved in the pathogenesis of ALS. Antioxidants are hypothesized to be effective in prevention and slowing down the progression rate of the disease. The aim of the present study was to systematically review current literature on the impact of vitamin E supplementation on ALS. PubMed, Scopus, Embase and Web of Science were searched for clinical trials until 10 March 2019 using the following keywords: "Amyotrophic lateral sclerosis", "ALS", "Immune disorder", "Vitamin E", "Tocopherols", "alpha-Tocopherol", "beta-Tocopherol", "gammaTocopherol" and "Antioxidant" without any filters activated. Two clinical trials and two cohort studies 
remained. Survival rate was considered as the primary outcome in clinical trials. And in cohort studies, ALS development in healthy subjects was considered. In clinical trials, vitamin E supplementation with the dose of 400 and $5000 \mathrm{mg} /$ day was not effective in slowing down the progression rate of the disease. But in both cohort studies, $50 \mathrm{mg}$ /day vitamin E supplementation was effective in prevention of ALS development, and the best result was seen in those who took vitamin $\mathrm{E}$ supplement regularly for more than 10 years. Vitamin E supplementation is effective in preventing ALS but it has no effect on slowing the progression rate of the disease. Further studies are needed to assess the effects of vitamin E supplement on disease progression in early stages of ALS.

\section{P148}

The Application of Transcranial Electrical Stimulation to Improve Alzheimer Symptoms: A Case Report

\section{Neda Mohammadi}

Department of Neuroscience, Faculty of Advanced Medical Sciences and Technologies, Shiraz University of Medical Sciences, Shiraz, Iran

The Neuroscience Journal of Shefaye Khatam, 2019; 7(3): P148

Alzheimer disease (AD) a multifactorial complex disorder is accompanied by decline of brain functioning, progressive cognitive deficit and can effect on mental abilities. Loss of memory and forgetfulness is known as a sign of $\mathrm{AD}$ onset. Therefore, the faster diagnosis and treatment of amnesia can prevent AD progress. Recent studies have suggested different ways such as meditation, exercise, trying new tasks to improve memory. In addition some studies have claimed that transcranial Direct Current Stimulation (tDCS) noninvasive technique is able to enhance memory and is safe for human use. In this case study we investigated the efficacy of the use of tDCS along with behavioral interventions in amnesia. The purpose of this study was to enhance the cognitive and memory functions of an individual by using tDCS. The patient was a 45-year-old woman and was chosen from clients who came to the neuroscience laboratory with a one-year history of memory problem. She had received 12 sessions of tDCS 3 times per week every other day. tDCS electrodes were placed based on the 10-20 EEG international system. Simultaneously, Cambridge brain sciences (CBS), Purdue pegboard test and Register maze memory test as behavioral interventions were used for memory and behavioral assessment and remediation. The referral was followed up for one year after tDCS application. The results of our study showed a positive effect of using combination of behavioral interventions and tDCS in order to improve memory and following that preventing AD. Further research is needed for study the effectiveness of $\mathrm{tDCS}$ for $\mathrm{AD}$.

\section{P149}

The Effect of HTLV-1 Virulence Factors (HBZ, Tax, Proviral Load), and Expression of CXCR3 and CCR6 and Their Ligands on Manifestation of HTLV-1 Associated Myelopathy Tropical Spastic Paraparesis

Sanaz Ahmadi Ghezeldasht, 2, Hanieh Tarokhian', Arman Mosavat, Hooshang Rafatpanah', Seyyed Abdolrahim Rezaee ${ }^{2}$

${ }^{1}$ Blood Borne Infections Research Center, Academic Center for Education, Culture and Research (ACECR), Mashhad, Iran

${ }^{2}$ Immunology Research Center, Department of Inflammation and Inflammatory Diseases, Mashhad University of Medical Sciences, Mashhad, Iran

${ }^{3}$ Kermanshah University of Medical Sciences, Kermanshah, Iran

The Neuroscience Journal of Shefaye Khatam, 2019; 7(3): P149

Introduction: Previous studies have suggested debatable roles of Tax and HBZ gene expression as virus factors and chemokines and chemokine receptors in host for induction of inflammatory response in HTLV1-associated myelopathy/tropical spastic paraparesis (HAM/TSP). In the present study HTLV-1 and host interactions, chemokine and chemokine receptors involved in trafficking of lymphocytes to the CNS were measured. Materials and Methods: A cross-sectional study was conducted on $33 \mathrm{HAM} / \mathrm{TSP}$ patients and 38 HTLV-1 asymptomatic carriers (ACs). Proviral load (PVL) were assessed using the quantitative real-time PCR (TaqMan), HTLV-1-Tax, HBZ gene expression, and, CCR6, and CXCR3 mRNA expression, and CXCL9 and CXCL10 protein levels and HLA-I were evaluated. Results: The HTLV-1 PVLs in HAM/TSP and ACs were $306 \pm 360.741$ copies/104 PBMCs and 250.98 \pm 629.94 copies/104 PBMCs, respectively; the PVL was higher in HAM/TSP than that in ACs $(p=0.004)$. HTLV-1 Tax and HBZ expression in HAM/TSP was higher than that in ACs, wherein only the Tax expression was statistically significant $(\mathrm{p}=0.039)$. In contrast to Japanese HTLV-1-infected subjects, HLA-A*02, HLA-A*24, HLA-Cw*08, and HLA-B*5401 did not exhibit preventive effects for HAM/TSP manifestation. CCR6 expression was higher in HAM/TSP patients and in ACs compared to the healthy controls $(\mathrm{P}=0.005$ and $\mathrm{P}=0.04$, respectively). A significant difference was observed in CCR6 expression when a combination of HAM/TSP patients and ACs were compared to the healthy individuals $(\mathrm{P}=0.005)$. Furthermore, there was a significantly lower CXCR3 expression between $\mathrm{HAM} / \mathrm{TSP}$ and control groups $(\mathrm{P}=0.001)$, and between the ACs and healthy controls $(\mathrm{P}=0.001)$. However, the increased CXCR3 expression in ACs compared to HAM/TSP patients was not significant. Furthermore, the CXCL10 protein levels in HAM/TSP patients was higher than in controls $(\mathrm{P}=0.012)$, and CXCL9 protein levels was also higher in the HAM/TSP and ACs 
groups than in the controls $(\mathrm{P}=0.001$ and $\mathrm{P}=0.004$, respectively). Conclusion: Overall, HTLV-1 PVL and Tax may be the valid predictors of disease development, but Tax is more helpful than PVL for the monitoring of HTLV-1-infected patients. It seems that decreased expression of CXCR3 and higher expression of CCR6 were associated with HTLV-1 infection, what indicate that these alterations may favor virus dissemination but not disease manifestation.

\section{P150}

The Effectiveness of Body Psychotherapy on the Amplitude of all Brain Waves of a Child with Tic Disorder: a Case Report

\section{Ali Ghanaei Chamanabad, Fatemeh Ghanaei}

Ferdowsi University of Mashhad, Mashhad, Iran

The Neuroscience Journal of Shefaye Khatam, 2019; 7(3): P150

People with a tic cannot stop their body from doing so. For example, a person with a motor tic might keep blinking over and over again. Recording of neuronal activity from multiple brain regions performed, this model during the expression of motor tics showed that tics are associated with phasic changes of neuronal activity throughout the Cortico Basal Ganglia pathway culminating in the disinhibition of the cortex and release of a tic. The similarity of obsessive-compulsive disorder with tick disorder has led to the suggestion that in both of the excessive disorder and the intensity of the waves in the Medial orbital frontal cortex and Basal ganglia are more than normal people. The purpose of this research is to determine whether Body psychotherapy (BP) can affect the reduction amplitude of the all brain waves. This research was conducted on a 10-year-old boy Which was diagnosed by a psychiatrist with motor tic disorder. brain waves were recorded in the different brain areas before the beginning of Body psychotherapy (BP) sessions by the neurofeedback. This session was held for 50 sessions 3 days a week and each session for an hour and thirty minutes. After completing this sessions, were recorded again from the same brain area and it was observed that significant decrease in the amplitude of all brain waves was created and also at the end of the sessions, there was no motor tic in the child. Further research is needed for study the effectiveness of Body psychotherapy (BP) for Tic disorder.

\section{P151}

The Protective Effect of Zataria Multiflora on the Embryotoxicity Induced by Bisphenol A in the Brain of Chicken Embryos

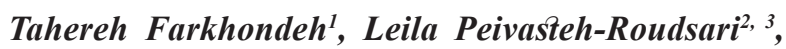
Saeed Samarghandian ${ }^{4,5}$

${ }^{1}$ Cardiovascular Diseases Research Center, Birjand University of
Medical Sciences, Birjand, Iran

${ }^{2}$ Department of Environmental Health, Faculty of Public Health,

Tehran University of Medical Sciences, Tehran, Iran

${ }^{3}$ Tehran University of Medical Sciences, Tehran, Iran

${ }^{4}$ Noncommunicable Disease Research Center, Neyshabur University of Medical Sciences, Neyshabur, Iran

${ }^{5}$ Department of Basic Medical Sciences, Neyshabur University of Medical Sciences, Neyshabur, Iran

The Neuroscience Journal of Shefaye Khatam, 2019; 7(3): P151

Introduction: Bisphenol A (BPA), is one of the main industrial chemicals synthesized for various purposes. Materials and Methods: In the present study, the brain tissue of chicken embryos was used to evaluate the embryotoxic effects of BPA and also the preventive effects of Zataria multiflora. To that end, fertile eggs were categorized into four groups $(n=10)$. After four days, BPA (200 ppm) was injected into the air sacs of the eggs of the experimental groups. The Zataria multiflora (100 and $200 \mu \mathrm{g} / \mathrm{egg}$ ) was administered into chick embryos 6 hours prior to administration of BPA. The control group simply was treated with olive oil. The eggs were placed in an incubator with a temperature of $37^{\circ} \mathrm{C}$ and a humidity of $63 \%$. After 20 days, the embryos were beheaded and the brains were gathered for biochemical measurements. Results: The findings indicated that Zataria multiflora (200 $\mu \mathrm{g} / \mathrm{egg})$ significantly reversed the increased levels of MDA $(p<0.05)$ and protein $(p<0.001)$ in the brain of BPA-exposed groupand also the decreased levels of total antioxidant and GSH as well as the activities of SOD and CAT in the brain of BPA-exposed group $(\mathrm{p}<0.01$, $\mathrm{p}<0.05, \mathrm{p}<0.05, \mathrm{p}<0.05$, respectively). Conclusion: Zataria multiflora reversed the toxic effects of BPA on the embryonic development stages of the brain via modulating oxidative stress.

\section{P152}

Curcumin-Loaded Nanoparticles Attenuate Cognitive Impairment and Astrocytes Activation in Chemical Kindling Model of Epilepsy

Seyed Raheleh Ahmadian', Shahram Ghasemi², Mona Hashemian, Atefeh Akbari ${ }^{2}$

${ }^{1}$ Student Research Committee, Babol University of Medical Sciences, Babol, Iran

${ }^{2}$ Faculty of Chemistry, University of Mazandaran, Mazandaran, Iran

The Neuroscience Journal of Shefaye Khatam, 2019; 7(3): P152

Introduction: Epilepsy is one of the most common chronic neurological disorders whose prevalence is significantly. Many different pro-inflammatory factors normally present in small amounts with in the brain and increase after pathologic complications including during epileptic seizures that can act as transient and longterm pathologic factors. So, use of anti-inflammatory 
agents such as curcumin has been regarded to reduce the epilepsy symptoms. But its medical application has been hampered due to low water solubility. To improve the aqueous solubility of curcumin, it has been loaded on chitosan -alginate nanoparticles (NPs). In this study, the effect of curcumin NPs on memory improvement and glial activation was investigated in pentylenetetrazol (PTZ)- induced kindling model. Materials and Methods: Male NMRI mice have received the daily injection of curcumin NPs at dose of 12.5 or $25 \mathrm{mg} / \mathrm{kg}$. All interventions were injected intraperitoneally (i.p), 10 days before PTZ administration and the injections were continued until $1 \mathrm{~h}$ before each PTZ injection. Spatial learning and memory was evaluated using Morris water maze test after the 7th PTZ injection. Animals have received 10 injections of PTZ and then, brain tissues were removed and immunostaining method was performed against NeuN and GFAP/Iba1 for assessment of neuronal density and glial activation respectively. Results: Behavioral results showed that curcumin NPs exhibit anticonvulsant activity and prevent cognitive impairment in fully kindled animals. The level of neuronal cell death and glial activation reduced in animals which have received curcumin NPs compared to those received free curcumin. Conclusion: To conclude, these findings suggest that curcumin NPs effectively ameliorate memory impairment and attenuate the level of activated glial cells in a mice model of chronic epilepsy.

\section{P153}

Anti-Autism Potential of Hypercin Using Surface Decorated Multi-Walled Carbon Nanotubes

\section{Sedigheh Khanjani Jelodar', Mohammadreza Bigdeli ${ }^{1}$, Akbar Hajizadeh Moghaddam ${ }^{2}$}

${ }^{1}$ Department of Animal Sciences, Faculty of Biological Sciences, Shahid Beheshti University, Tehran, Iran

${ }^{2}$ Department of Biology, Faculty of Basic Sciences, University of Mazandaran, Babolsar, Iran

The Neuroscience Journal of Shefaye Khatam, 2019; 7(3): P153

Introduction: Although the cause of autism remains unknown, both genetics and early postnatal environmental exposures, are known to cause ASD. Daily maternal separation during the postnatal period appears to be stressful to the pup and leads to changes in many behavioral, such as impaired social interactions, learning, and enhanced repetitive or stereotyped behavior as well as the impairment of neurogenesis. Materials and Methods: Newborn rats in all group expect control group subjected to individual isolations from their mother and nest for $1 \mathrm{~h}$ per day from postnatal days $1-9$. From the end of the lactation day, rats were treated with Hypersin and Hypersin Carbon nanotubes for two weeks. From postnatal days 36-40, we carried out neurobehavioral tests. Results: Here, we reported that newborn rats subjected to individual isolations from their mother and nest for $1 \mathrm{~h}$ per day from postnatal days 1-9 displayed apparent autistic-like symptoms including social deficits, excessive repetitive selfgrooming behavior, and increased anxiety- and impaired in learning and memory tested in young adult (postnatal days 36-40) compared to normal controls. Furthermore, these behavioral changes were accompanied by increase in neuroinflammation and impaired adult hippocampal and cerebellum neurogenesis. More importantly, chronic administration of carbon nanotube loaded hypercin, a clinically used anti-depression and mood stabilizer, decreased neonatal isolation-induced autistic-like behaviors, and neuroinflammation restored cerebellum neurogenesis $(p<0.01)$. Conclusion: This study indicate that carbon nanotube loaded hypercin may be a potential therapeutic agent against autism spectrum disorders (ASD) during development and carbon nanotubes are nanotechnology-enabled drug delivery systems (DDS) have emerged as promising agents to host, protect and transport the loaded drug to the target site at the required rate.

\section{P154}

\section{New Rehabilitation Management of Multiple Sclerosis}

\section{Salman Nazary-Moghadam}

Faculty of Paramedical Sciences, Mashhad University of Medical Sciences, Mashhad, Iran

The Neuroscience Journal of Shefaye Khatam, 2019; 7(3): P154

Introduction: Chronic neurodegenerative conditions, such as MS, result in a loss of physical and cognitive function from the destruction of neurons and from a lack of activation of the affected systems. People with MS experience physical and cognitive impairments potentially leading to inactivity and resultant deconditioning. This often becomes a cycle that is difficult to break. One question that frames the rehabilitation strategy chosen is whether the focus should be compensation for or restoration of lost function. Materials and Methods: Compensation includes interventions such as wheelchairs or walkers to assist with mobility or braces for absent or inadequate muscle power. Restoration is aimed at increasing the capacity of the system-for example, maximizing cardiovascular endurance by increasing maximal oxygen uptake or restoring full ROM. Results: Prescribing programs, activities, and exercises that provide an adequate stimulus to produce adaptation is critical to restore function or improve motor and cognitive performance. Although each patient case is unique, the most likely answer is that both strategies will be employed. The challenge for rehabilitation teams is to sort out how much of a 
patient's dysfunction arises from neurodegeneration, which necessitates compensation, and how much occurs from inactivity and system deconditioning, in which case system capacity can be restored to some extent. Conclusion: Rehabilitation professionals must choose therapeutic interventions based on whether compensation or restoration is the goal.

\section{P155}

Evaluation of the Relationship Between OSA Signs and Carotid Artery Atherosclerotic Characteristics Using Stop-BANG Questionnaire

\section{Vahid Mahdavizade ${ }^{1}$, Shokat Nazemian ${ }^{2}$, Fariborz Rezaeitalab ${ }^{3}$}

'Student Research Committee, Mashhad University of Medical Sciences, Mashhad, Iran

${ }^{2}$ Mashhad University of Medical Sciences, Mashhad, Iran

${ }^{3}$ Department of Neurology, Faculty of Medicine, Mashhad University of Medical Sciences, Mashhad, Iran

The Neuroscience Journal of Shefaye Khatam, 2019; 7(3): P155

Introduction: Obstructive sleep apnea (OSA), the most prevalent sleep related breathing disorder is characterized by partially or complete airflow cessation during sleep. Several studies have shown that there is a relationship between OSA and the risk of stroke. OSA, especially if accompanied by loud snoring, is proposed to be a risk factor for developing carotid artery sclerosis, which can be measured by carotid intimamedia thickness (IMT). This study aimed to examine the association between the risks of OSA achieved by STOP-BANG questionnaire, and IMT in patients with ischemic stroke. Materials and Methods: Patients with ischemic stroke enrolled in a cross sectional study. Every patients underwent ultrasound evaluation of carotid arteries of both sides by a neuro-sonologist. The patients were asked to answer to the standard questionnaire of STOP-BANG, which assesses the risk of OSA through eight Yes/No questions. Three or more positive responses to this questionnaire is considered as OSA and more positives is associated with higher risks of OSA. Results: Our study was comprised of 119 participants, $73(61.3 \%)$ of which were male and $46(38.7 \%)$ were female. The mean of Right IMT in the group who had apnea was 0.008 less than those who did not have apnea and this difference was significance. $(\mathrm{P}=0.047)$ Among the 66 subjects who had apnea, 25 $(37.9 \%)$ had $50-70 \%$ stenosis and $7(10.6 \%)$ had a stenosis of more than $70 \%$. The results of the Fisher's exact test showed a significant relationship between apnea and stenosis of carotid artery $(\mathrm{P}<0.001)$. Conclusion: The present study showed that there was a higher Right and Left IMT in apneic patients independent of the risk factors. Apnea was also shown to be related to carotid artery stenosis.

\section{P156}

PuraMatrix Hydrogel Improved Proliferation of Motor Neuron-Like Cells

Marzieh Darvishi 1, 2, Ardeshir Moayeri', Hatef Ghasemi Hamidabadi, 4, Taki Tiraihi', Javad Mirnajafi-Zadeh', Amir Shojaei, Farzane Abbasi Nezhad', Sajad Sahab-Negah ${ }^{7}$

${ }^{1}$ Department of Anatomy, Faculty of Medicine, Ilam University of Medical Sciences, Ilam, Iran

${ }^{2}$ Shefa Neuroscience Research Center, Khatam Alanbia Hospital, Tehran, Iran

${ }^{3}$ Department of Anatomy and Cell Biology, Faculty of Medicine, Mazandaran University of Medical Sciences, Sari, Iran

${ }^{4}$ Immunogenetic Research Center, Department of Anatomy and Cell Biology, Faculty of Medicine, Mazandaran University of Medical Sciences, Sari, Iran

${ }^{5}$ Department of Anatomical Sciences, Faculty of Medical Sciences, Tarbiat Modares University, Tehran, Iran

${ }^{6}$ Department of Physiology, Faculty of Medical Sciences, Tarbiat Modares University, Tehran, Iran

${ }^{7}$ Neuroscience Research Center, Mashhad University of Medical Sciences, Mashhad, Iran

The Neuroscience Journal of Shefaye Khatam, 2019; 7(3): P156

Introduction: Development of neural tissue engineering approach for differentiation of stem cells into spinal motor neurons in the injury site is a new perspective for neurodegenerative diseases. Numerous scaffolds produced from a variety of biomaterials can be used to improve cell differentiation in the field of tissue engineering. Materials and Methods: We obtained neural stem cells-derived motor neuron-like cells (MNLCs) from rat adipose tissue and evaluate proliferation and differentiation on $2 \mathrm{D}$ and 3D (PuraMatrix hydrogel) culture systems. Results: Our results indicated that $3 \mathrm{D}$ environment increase in the expression of mature motor neuron markers. Also results of the current study showed that proliferation and adhesion of MNLCs in 3D environment is more than 2D. Conclusion: Taken together, this study suggested that $3 \mathrm{D}$ environment can be considered as a potent therapeutic option for neural tissue regeneration.

\section{P157}

Effect of Intraperitoneal Injection of Conditioned Medium of Mesenchymal Stem Cells in the Chronic Constriction Injury (CCI) Model of Neuropathic Pain in Male Rat

Samad Nazemi', Mahtab Helmi², Mohammad Shafie Mojaddadi $^{1}$, Marzieh Kaffami ${ }^{1}$

${ }^{1}$ Cellular and Molecular Research Center, Faculty of Medicine, Sabzevar University of Medical Sciences, Sabzevar, Iran ${ }^{2}$ Student Research Committee, Sabzevar University of Medical 
Sciences, Sabzevar, Iran

The Neuroscience Journal of Shefaye Khatam, 2019; 7(3): P157

Introduction: Neuropathic pain is one of the most important chronic pain results from injury to nervous system. Inflammatory mediators are involved in creating neuropathic pain. Mesenchymal stem cells (MSCs) by having anti-inflammatory properties can be a potential option in alleviating or treatment of neuropathic pain. Hence, this study aimed to investigate the effect of MSCs supernatant on neuropathic pain in chronic constriction injury (CCI) model. Materials and Methods: In this study, 24 male Wistar rats (6-8 weeks old, 200-250 gr) was used and randomly divide in 3 experimental group (Sham, CCI+MSCs and CCI+DMEM). MSCs were isolated from rats' tibia bone marrow and cultured in laboratory. The MSCs supernatant was harvested at passage 5 . Neuropathic pain was induced by CCI method. Experimental group received intraperitoneally $1 \mathrm{ml}$ of MSCs supernatant on days $-1,1$ and 2. Control group received DMEM as the same manner. In all groups, mechanical allodynia and thermal hyperalgesia was performed on days $-1,2,6,10$ and 14 . The results were analysis by mixed mode31 ANOVA $(\mathrm{P}<0.05)$. Results \& Conclusion: The results of behavioral studies revealed that in MSCs group allodynia and hyperalgesia significantly decrease in comparison to DMED group, on day 6 after CCI and this effect was extending until day 14. It seems that preemptive injection of MSCs supernatant can be effective in prevention of neuropathic pain in CCI induced neuropathic pain in rat.

\section{P158}

Compare the Resilience of Multiple Sclerosis Patients with Healthy People in Ahwaz

\section{Khadijeh Bazyari}

Department of Clinical Psychology, Payam Noor University, Ramshir, Iran

\section{The Neuroscience Journal of Shefaye Khatam, 2019; 7(3): P158}

Introduction: The aim of this research was to compare the resilience of MS patients with healthy people in Ahwaz. Materials and Methods: 100 people (50 patients and 50 healthy people) were selected by convenience sampling. Measurement instruments include the Multiple Sclerosis Resilience Scale for Adults (RSA) for Stressful Situations. Data were analyzed using (ANOVA) and multi analysis of variance (MANOVA). Results: Results showed there is no significant difference between resilience $(\mathrm{F}=. / 887, \mathrm{P} . / 349)$ of $\mathrm{MS}$ patients and healthy people. Conclusion: increasing resilience may help individuals overcome stressful and traumatic events and experience quality of life with disability. Due to the effects of resiliency on mental and physical health of MS patients, the more researches need in this area.

\section{P159}

Training Performance's Satisfaction of Empowered Nurses from Stroke Patients Viewpoints

\section{Nasrollah Alimohammadi ${ }^{1}$, Fariba Taleghani ${ }^{2}$, Zahra Aslani $^{3}$, Shayan Alijanpou ${ }^{3}$}

${ }^{1}$ Nursing and Midwifery Care Research Center, Faculty of Nursing and Midwifery, Isfahan University of Medical Sciences, Isfahan, Iran

${ }^{2}$ Department of Adult Health Nursing, Faculty of Nursing and Midwifery, Isfahan University of Medical Sciences, Isfahan, Iran ${ }^{3}$ Students Research Committee, Faculty of Nursing and Midwifery, Isfahan University of Medical Sciences, Isfahan, Iran

The Neuroscience Journal of Shefaye Khatam, 2019; 7(3): P159

Introduction: Stroke is a major cause of disability worldwide. Some studies have shown that nurses do not have sufficient knowledge and skills to educate and manage such patients. So, patients suffer from many problems. We investigated the effects of the nursing empowering on stroke patients satisfaction. Materials and Methods: This cross-sectional study was conducted on 29 patients with stroke who had referred to Alzahra hospital in Isfahan during 2013-2014 with the simple method. Workshops, pamphlets and booklet were used for empowering nursing. The empowered nurses trained stroke patients and finally patients filled in the satisfactions questionnaire at the time of discharge. The data gathering tool was a researchermade that consists of characteristics of patients, the participants' satisfaction with self-care training and nurses' performance. Data were analyzed by T-test. Results: 27 cases $(93.10 \%)$ mentioned that "they would try to follow all the training which received from nurses". 23 cases $(73.10 \%)$ were satisfied with self-care training. Majority of patients, 25 participants (85\%) were satisfied with the nurses' training performance and 24 cases $(82.80 \%)$ were highly satisfied with the nurses training method. Conclusions: Most patients were satisfied with self-care training held by nurses. From stroke patients' viewpoints, the nurses' performance and method were associated with high satisfaction. Therefore, improving nurses' training role by empowering nurses' is suggested to increasing patient's satisfaction.

\section{P160}

Nanocurcumin Decreases Pro-Inflammatory Mirnas Expression Profile in Multiple Sclerosis

\section{Sanam Dolati, ${ }^{1,2}$}

${ }^{1}$ Department of Immunology, Faculty of Medicine, Tabriz University of Medical Sciences, Tabriz, Iran

${ }^{2}$ Aging Research Institute, Tabriz University of Medical Sciences, Tabriz, Iran 
The Neuroscience Journal of Shefaye Khatam, 2019; 7(3): P160

Introduction: Multiple sclerosis (MS) is a demyelinating inflammatory disorder of the central nervous system. The dysregulated expression of miRNAs has been established to be accompanying with diverse pathologies of autoimmune diseases such as MS. The aim of the current study was to assess the effects of nanocurcumin on the expression levels of pro-inflammatory miRNAs in PBMCs derived from MS patients. Materials and Methods: In this study we investigated the miRNA expression profiles in 35 healthy controls, and from 25 patients at baseline and after 6 months of nanocurcumin treatment and also from 25 patients whose received placebo by real-time PCR. Results: Our data revealed that the expression of miR-16, miR-17-5p, miR-17-92, miR-29b, miR-128, miR-145, miR-155, miR-326, miR-550, and miR-340 was significantly increased in CD4+ T cells of untreated RRMS patients compared with healthy controls. The expression of this pro-inflammatory miRNAs including miR-16, miR-17-92, miR-29b, miR-128, miR-132, miR-145, miR-155, miR-326, miR-550, and miR-340 was significantly decreased in nanocurcumin-treated RRMS patients $(p=0.004 ; p=0.03 ; p=0.003 ; p=0.009$; $p=0.001 ; p=0.0009 ; p=0.0006 ; p=0.0007 ; p=0.0003$; $\mathrm{p}=0.001)$ compared with placebo group. Conclusion: This is a new finding that might represent the possible involvement of these miRNAs to MS pathogenesis. In this clinical trial, it was found that the six-month oral administration of nanocurcumin could decrease mRNA expression levels of pro-inflammatory miRNAs in MS patients. Our results established that nanocurcumin is an effective agent for the treatment of MS patients with immune cell abnormalities.

\section{P161}

Effect of Induction of Spreading Depression by KCL on Caudate Nucleus Firing Pattern: A Single Unit Recording Study

\author{
Abedi $M^{1}$, Nemati $A^{2}$, Vafaee F1, Abbassian $H^{1}$ \\ ${ }^{1}$ Department of Neuroscience, Faculty of Medicine, Mashhad \\ University of Medical Sciences, Mashhad, Iran \\ ${ }^{2}$ Department of Reconstructive Dentistry, Faculty of Dentistry, \\ Mashhad University of Medical Sciences, Mashhad, Iran
}

The Neuroscience Journal of Shefaye Khatam, 2019; 7(3): P161

Introduction: Spreading depression (SD), a phenomenon with ionic and hemodynamic changes in the brain. Studies into this experimental event provides evidence for its involvement in brain pathophysiology. SD plays an important role in some clinical disorders including migraine, cerebrovascular diseases and amnesia. However, further studies are needed to demonstrate the involvement of SD in these clinical disorders. In this study we aim to see what possible changes may occur in firing pattern of caudate nucleus in single cell recording invivo. Materials and Methods: Rats (aged 16-20 weeks) provide from animal house of medical school of Mashhad. Experiments done under ketamine plus xylazine (100 and $20 \mathrm{mg} / \mathrm{kg}$ i.p.) anesthesia and application of KCL solution on cerebral surface. For recording EEG, 3 screws, one in frontal and 2 in posterior parieto-occiput area using "elab, Science Beam instruments. Iran partodanesh co.” apparatus was used. The electroencephalogram was filtered between 1 and $100 \mathrm{~Hz}$ and was continuously monitored via an analog monitor scope. For single cell recording from caudate neurons, the electrode lowered through a small burr hole in the skull by Stereotactic coordinates: (AP= 1.2, $\mathrm{L}=2.2$ and $\mathrm{DV}=4$ ). The electrode after isolating active neuron, subdural injection of KCL $0.2 \mathrm{ml}$ at 15 minutes of session performed for each rat via a foramen in skull between EEG electrodes in fronto-parital area and firing rate was recorded for a subsequent period of up to $60 \mathrm{~min}$. Unit records filtered between 300 and 3k Hz. Results: Data shows application of KCL take vigorous effect on firing frequency and some aspect of pattern like increase frequency and amplitudes. Also some very fast burst firing after minutes of KCL seen. Conclusion: This study shows very direct and fast link between stimulation of cortex and caudate nucleus.

\section{P162}

Pregnant Effect on Multiple Sclerosis: A Case Report

\section{Mohammad Reza Khojasteh ${ }^{1,2}$, Abbas Norian ${ }^{3}$}

${ }^{1}$ Mashhad Branch, Islamic Azad University, Mashhad, Iran

${ }^{2}$ Student Research Committee, Mashhad Branch, Islamic Azad University, Mashhad, Iran

${ }^{3}$ Department of Neurology, Islamic Azad University, Mashhad, Iran

The Neuroscience Journal of Shefaye Khatam, 2019; 7(3): P162

A 46 housekeeper woman who had 3 children 17 years ago refers to neurologist with insensibility, depression, dysphagia, spastic hemiparesis and central hemiplegia complaint. After neurologist examination and MRI she hospitalized in Aria Hospital for 7 days. She release hospital with Multiple Sclerosis (MS) diagnosis and Rebif were started for her. In 1 years follow up she had more tireless and forgetfulness some times. 2 years after MS diagnosis she was get unwanted pregnancy; for ethical reasons she cannot abort the child. In fives month of pregnancy she punctuate all drug without any consulting. In 45 th week of pregnancy labor a normal baby. After child birth she just had lumbago and pain in foots but all other complications were effectively reduce. She only mentions urinary incontinence. The patient started the simvastatin two years after giving birth and did not mention a particular problem. A 
year later she afflicted asthma. Two years later, with paresthesia, pain in the lower member of body and headache with the possibility of recurrence of the MS disorder she referenced to neurologist. The patient said she has not taken his medicines regularly. She was under medical treatment again. She is getting better with the use of medications and as soon as the drug is stopped, the symptoms of the patient recurrence again. The patient did not referring to the neurologist for 3 years and discontinues his treatment in a crosssectional manner at his own discretion. After this time, with headache, nausea, photophobia, and lumbar pain, she were referred to a doctor again and get treatment with amitriptyline, simvastatin and calcium drugs. The patient was better with taking medication to now, but as soon as the drug is stopped, she experiences all Previous symptoms.

\section{P163}

Identification of New Causative Genetic Mutations of Familial Early Onset Parkinsonism in Iranian Population Using Next Generation Sequencing Technique (NGS)

\section{Mohammad Soudyab, Ariane Sade-Nabavi}

Department of Medical Genetics, Mashhad University of Medical Sciences, Mashhad, Iran

The Neuroscience Journal of Shefaye Khatam, 2019; 7(3): P163

Introduction: Parkinsonism is a chronic disorder with considerable burden worldwide. This neurogenetic disease is the most common movement disorder and the second most common neurodegenerative disorder after Alzheimer disease. The approximate prevalence of Parkinsonism is reported to be 572 per 100,000 individual older than 45 years of age. The cardinal manifestations include muscle rigidity, bradykinesia, postural instability, and resting tremor. Parkinsonism's manifestations occur before the age of 50 in about $10 \%$ of patients. Genetic causes play a major role in Parkinson and account for up to $15 \%$ of cases. Moreover, most of the hereditary forms of Parkinson categorized in the early-onset group. However, most of the genetic causes are still unknown. So, finding the genetic basis of earlyonset Parkinsonism will be helpful in determining the true nature of this disorder and providing new insights for further therapies in the near future. Material and Methods: Forty-one familial early-onset Parkinson patients who were referred to the genetic clinic of Ghaem hospital were enrolled in the present study. After taking a brief history and performing a complete neurologic physical examination by a neurologist, the patient's pedigree drowns by a geneticist. Only the patients who had at least 2 family members with Parkinsonism will be included and those patients with a history of head trauma or using any medication were not included. Whole exome sequencing with the depth of $150 \mathrm{X}$ will be performed for each patient and homozygosis mapping will be performed for each family. Then, the putative variants will be confirmed by Sanger sequencing and segregation analysis. Results: In the six families that have been studied so far, we found two completely pathogen mutations in the known genes of this disease (PARK2 \& PINK1). There are also several variants of unknown significance (VUS) that have a strong correlation with patient phenotypes. In one of our families, we found a mutation in a new possible causative gene that has not been reported in studies. All mutations were confirmed by analysis in families. Conclusion: Better understanding the etiology of this disease helps to better diagnose and treat it. Due to the high rate of consanguineous marriage in Iran, there are many undiscovered recessive genes that make it difficult to genetic cause's diagnosis. genetic causes of a high proportion of Iranian families are not recognized by present genetic panels. The aim of this study is to make patients' genetic recognition panels more complete and to find new therapeutic targets.

\section{P164}

Dose-Dependent Protective Effects of Diazepam on Ischemic Hypoxic Rats Brain

\section{Akbari Rad $\mathrm{M}^{1}$, Abbassian $\mathrm{H}^{2}$}

${ }^{1}$ Department of Internal Medicine, Faculty of Medicine, Mashhad University of Medical Sciences, Mashhad, Iran

${ }^{2}$ Faculty of Medicine, Mashhad University of Medical sciences, Mashhad, Iran

The Neuroscience Journal of Shefaye Khatam, 2019; 7(3): P164

Introduction: Normal neuronal function depends on the integration between a high and low level of membrane potential and firing thresholds witch integrated by excitatory and inhibitory neurotransmitters. GABA receptors play inhibitory roles in the central nervous system by ligand-gated chloride channels. Diazepam is a medication which acts on GABA receptors allosterically and can lower excitability of neurons via altering chloride channel gating phases. One of the potential Targets for Neuroprotection is GABA receptor activators, thus we investigate the comparative effects of increasing doses of Diazepam in the present study. Materials and Methods: Wistar male Rats weighing about 250-350g and were kept in standard condition, are chosen for the current study. The animals were divided into 5 groups of 8 including Group 1, Sham-operated without induction of ischemia. Group 2, Negative control with application brain global ischemia and $1 \mathrm{mg}$ normal saline injected intraperitoneal. Group 3, 4, and 5 were injected Diazepam intraperitoneal 2, 5 and $10 \mathrm{mg} /$ $\mathrm{kg}$ respectively. Brain global ischemia was done using Four Vessel Occluding (4VO) method expatiated by 
Pulsinelli with some modification. Tissue prepared After 72 hours by removing brains and fixed in formaldehyde $10 \%$. Slices (2-4 Microns) of tissues stained for H\&E. Results: Damaged neuronal cells were seen in the various area of brain tissues. Necrosis cells were seen in the cerebellum, basal ganglia, and cortex. In normal saline injection, ischemia and necrosis of hippocampal area CA1-4 occurred. In Diazepam injected groups, significant decreasing neuronal damages according to pathologic criteria such as degrees of ischemic markers are seen in light microscopic fields, but the results were not dose-related. Conclusion: Our results demonstrated that administration of Diazepam can improve the survival of ischemic cells in brain tissues. Further investigations especially in tissue and molecular level needed to survey possible dose-dependent changes.

\section{P165}

Neuroprotective and Therapeutic Effects of Garlic Extract on Midbrain Dopaminergic Neuron in 6OHDA-Induced Rat Model of Parkinson's Disease

\section{Maryam Bigham ${ }^{1}$, Abbas Mohammadipour ${ }^{1}$, 2, Mahmoud Hossaini, Alireza Ebrahimzadeh- Bideskan ${ }^{1, \quad 2}$}

'Department of Anatomy and Cell Biology, Faculty of Medicine, Mashhad University of Medical Sciences, Mashhad, Iran

${ }^{2}$ Microanatomy Research Center, Faculty of Medicine, Mashhad University of Medical Sciences, Mashhad, Iran

The Neuroscience Journal of Shefaye Khatam, 2019; 7(3): P165

Introduction: Parkinson's disease is an age-related disorder characterized by a progressive degeneration of dopaminergic neurons of Substantia nigra(pc). On the other hand, Garlic Extract is known for its anti-oxidative and anti-inflammatory effects. It also increases important neurotrophic factors of brain such as BDNF and NGF. The aim of this research was to evaluate neuroprotective and therapeutic effects of Garlic Extract on density of dopaminergic neurons in 6OHDA-induced model of Parkinson's disease. Materials and Methods: 30 male Wistar Rats were divided randomly into 3 groups. 6OHDA group: After one week of Normal Saline administration, $60 \mathrm{HDA}(80 \mathrm{Mg} / \mathrm{kg})$ was injected into the left MFB by mean of Hamilton syringe. Neuroprotective group: After one week of Garlic Extract administration, 6 OHDA $(80 \mathrm{Mg} / \mathrm{kg})$ was injected into the left MFB by mean of Hamilton syringe. Therapeutic group: After one week of Garlic Extract administration, 6OHDA $(80 \mathrm{Mg} / \mathrm{kg})$ was injected into the left MFB by mean of Hamilton syringe. Administration of Garlic Extract was continued for one week after surgery. Apomorphineinduced rotational test was performed two weeks after 6OHDA injection in order to confirm parkinsonian model. Behavioral tests such as Open field, Rotarod, Hanging and Pole were performed in order to assess the motor impairments four weeks after surgery. Shuttle box test was performed in order to assess the memory six weeks after surgery. Results: It is expected to improve motor impairments and memory in Neuroprotective and Therapeutic groups compared to 6OHDA group. In addition, dopaminergic neurons were increased in Neuroprotective and Therapeutic groups compared to 6OHDA group. Conclusion: After behavioral tests, density of dopaminergic neurons in Substantia nigra were detected and calculated by IHC staining and stereological methods respectively in different groups.

\section{P166}

Bioinformatics Evaluation and Study of Mirnas Regulatory Effect on Degenerative and Cancerous Diseases

Masoumeh Massah ${ }^{1,2}$, Reza Basiri Kejany, Maryam Jalali $^{4}$, Mansoureh Azadeh

${ }^{1}$ Department of Biochemistry, Islamic Azad University, Najafabad, Iran

${ }^{2}$ Researcher Biotechnology Institute, Department of Biotechnology, Nonprofit University of Ashrafi Esfahani, Esfahan, Iran

${ }^{3}$ Department of Basic Sciences, Najafabad Branch, Islamic Azad University, Najafabad, Iran

${ }^{4}$ Department of Biochemistry, Najafabad Branch, Islamic Azad University, Najafabad, Iran

${ }^{5}$ Zist Fanavary Novin, Biotechnology Institute, Department of Biochemistry, Islamic Azad University, Najafabad, Iran

The Neuroscience Journal of Shefaye Khatam, 2019; 7(3): P166

Introduction: Neurodegenerative diseases include parkinson, Multiple Sclerosis (MS), Amyotrophic Lateral Sclerosis (ALS) and Alzheimer that expect for the rest, it occurs as Alzheimer disorder in the muscles and the limbs. In addition, ALS and MS are autoimmune disease. These disease are progressive and with its development in various organs, looking for many psychological complications including depression. Their prevalence its increasing in the world and unfortunately, so far no definitive treatment has been obtained for them. MicroRNAs are a group of small non-coding RNAs that involve in regulation processes by translation inhibition. They binding to complementary sequences located in 3UTR of target mRNA. Materials and Methods: The databases of articles NCBI, MalaCards was obtained from the genes involved in these diseases in this study, databases such as miRBase, miRNASNP, MiRWALK2 and DAVID were used. Results: According to the data, were evaluated 10 genes have single-nucleotide polymorphisms (SNP)s with high frequency, and 25 miRNAs for each disease. The results of the bioinformatics data showed that of these, 2miRNA: has-miR-548d-5p and $3 p$ and has-miR$3117-3 p$ and $5 p$ have the maximum power to regulate the genes desired. Example of these genes are parkinson: GBA, TBP, MAPT, GLID2, LRRK2, Alzheimer: SOX9, 
SOX2, APP, A2M, PLAU, MS: MBP, IL7R, HLADRB1, PDCD1, CCR5, ALS: SOD1, FUS, DCTN1, TBK1, C9ORF72. Then it was determined by checking the directions that theirs is a close relationship between the genes of these disease together, as well as cancer genes, it also affects important cancer genes such as $\mathrm{K}-\mathrm{Ras}, \mathrm{MDM} 2$. In addition, they also affect the genes that activate the immune system such as GRB2, CAN, NFAT. Conclusion: According to the results, it can be predicted that these miRNAs are effective in controlling the expression of genes involved in neurological diseases, different types of cancers especially Leukemia as well as immune system diseases.

\section{P167}

New Approaches in Rehabilitation of Parkinson Disease

\section{Afsaneh Zeinalzadeh \\ Faculty of Paramedical Sciences, Mashhad University of Medical Sciences, Mashhad, Iran}

The Neuroscience Journal of Shefaye Khatam, 2019; 7(3): P167

Introduction: Physical rehabilitation is effective in the treatment of people with Parkinson disease. The results are greater when treatment is started early. Incorporates complex, sequential movements with multiple sensory inputs creates responses that are environmentally appropriate, challenges balance, uses large-amplitude movements, and is effective. Animal research indicates that exercise may protect the dopaminergic neurons. Materials and Methods: First, the activities selected must engage the patient: the patient must find the activities interesting enough to do them regularly. Variety is important to facilitate shifts in movement as well as in thought. And movements must be big! (In fact, one treatment technique even uses that word in its name.) Activities that are designed to improve balance are valuable even in the early stages of the disease. Programs that emphasize sensory-motor integration, agility, and motor learning demonstrate decreased progression of disease and improved motor function. Programs that involve the coordination of dual motor-cognitive tasks and complex sequences of movements and that force the participant to quickly change movements. Results: As the person progresses, practice of precise ADLs is advisable. These include rising from a chair, getting out of bed, turning in bed, adjusting covers, and being aware of posture. At the later stages of the disease, breathing exercises will need to be a more prominent aspect of treatment. Conclusions: Big movements still need to be stressed. At these later stages of the disease, use of assistive equipment may also need to be taught. What follows are ideas for treatment of more specific aspects of the disease. These are ideas and are not exhaustive. The words big, fun, and novel are good words to remember when planning treatment.

\section{P168}

The Study of How to Articulate the Expression of Key Genes in Alzheimer's Disease (AD) Bioinformatics Approaches and Analyzing Pathways Involved in Them

Masoumeh Massah", 2, Nioosha Ataee ${ }^{2}$, Niloofar Ataee $^{3}$, Sara Nezarati Zadeh ${ }^{1}$, Samira Rahimirad ${ }^{4,5}$, Mansoureh Azadeh ${ }^{1,2}$

${ }^{1}$ Department of Biochemistry, Najafabad Branch, Islamic Azad University, Najafabad, Iran

${ }^{2}$ Department of Biotechnology, Nonprofit University of Ashrafi Esfahani, Esfahan, Iran

${ }^{3}$ Department of Genetics, Falavarjan Branch, Islamic Azad University, Esfahan, Iran

${ }^{4}$ Zist Fanavary Novin, Biotechnology Institute, Esfahan, Iran

${ }^{5}$ Parand Branch, Islamic Azad University, Parand, Iran

The Neuroscience Journal of Shefaye Khatam, 2019; 7(3): P168

Introduction: $\mathrm{AD}$ is a Multifaceted cognitivebehavioral disorders disease and its prevalence is increasing in today's societies. $\mathrm{AD}$ is due to the accumulation of a myloid proteins in the form of neurofibricular cavities (NFT) inside the neurons, and eventually disruption occurs in the cerebral blood flow. The patient suffers from a lack of memory, cognitive and behavioral abilities. Because of the disorder in life, treatment is of great importance. Materials and Methods: By sites PubMed, Google scholar and MalaCards identified key genes of AD. Then the biology pathways of these genes were obtained and evaluated by NCBI. To investigate the role of miRNAs in AD miRWALK2.0 and miRNASNP databases were served to predict miRNA-target genes interactions. Results: The data showed that SOX9, SOX2 and CR1 genes are important in CNS that mutation causes oxidative stress, inflammatory processes and NFT generation. According to bioinformatics databases in SOX9 selected 3 single-nucleotide polymorphisms (SNP): rs1042673, rs1042667, rs74999341 and 3miRNA: hsa-let-7d-3p and $-5 \mathrm{p}$, has-miRNA-1181, hsa-miR-3117-3p and 5p. In SOX2 selected rs191376028 and hsa-miR-548d-5p and 3p. In CR1 selected 3SNP: rs47274776, rs66149234, rs41274776 and 3miRNA: hsa-miR-548o-3p, hsamiR-665, hsa-miR-1323. The most Pathways biological include SOX9: cAMP and Hippo signaling organism specific biosystem, CR1: Legionellosis conserved biosystem and Hematopoietic cell lineage organism specific biosystem, SOX2: Regulating pluripotency of stem cells organism specific biosystem. Then the best interconnection networks were draw up. Conclusion: One can predict that by increasing the performance of these miRNAs can be set correctly biological pathways. 
Combined Estrogen and Progesterone Effect on Inflammatory Cytokines Gene Expression and Lymphocyte Migration to Penumbra Region after Ischemic Stroke Model in Rat

\section{Zahra Alibolandi', Abolfazl Azami Tameh ${ }^{2}$}

${ }^{1}$ Student Research Committee, Kashan University of Medical Sciences, Kashan, Iran

${ }^{2}$ Anatomical Sciences Research Center, Kashan University of Medical Sciences, Kashan, Iran

The Neuroscience Journal of Shefaye Khatam, 2019; 7(3): P169

Introduction: Ischemic stroke is the most leading cause of death and long-term disability due to middle cerebral artery occlusion, which result in inflammation process in the affected area of the brain. Although many attempts have been made to treat stroke, therapeutic modalities to fight stroke are still limited.However, mounting evidence have documented that estrogen and progesterone hormones have neuroprotective effects. In the present study the effect of combined dose of estrogen and progesterone on gene expression level of inflammation cytokine including TNF-a, IL-6 and IL-1B as well as the migration of lymphocytes to the marginal region, in the MCAO model have been investigated. Materials and Methods: In this study, 40 adult male Wistar rats were kept in a controlled environment and then randomly divided into three groups: ischemia, hormone and sham. The MCAO model was performed within $1 \mathrm{~h}$ and estrogen and progesterone combination were immediately injected after MCAO subcutaneously. TTC staining was used for measurement of infarct volume. Moreover, IHC technique carried out in order to evaluate lymphocyte migration rate to pneumobra region. Besides, real-time PCR and western blot analysis was used to explore gene and protein expression level of TNF- $\alpha \cdot$ IL- $1 \beta$ and IL- 6 respectively. P value less than 0.5 was statically considered significant. Results: Combination of estrogen $(25 \mu \mathrm{g} / \mathrm{kg})$ and progesterone (10 $\mathrm{mg} / \mathrm{kg}$ ) injected immediately after focal ischemia could significantly reduce lesion volume, migration of CD4 +lymphocytes to pneumobra region and thus reduced inflammation. It also significantly increased the score of behavioral tests. Conclusion: The results of this study proved the anti-inflammatory effect of combined dose of estrogen and progesterone. Furthermore, the findings indicated such combination significantly reduces inflammation and migration of CD4 +lymphocytes to pneumobra region after $\mathrm{MCAO}$

\section{P170}

Neurotoxic Effects of Stanozolol on Male Rats' Hippocampi: Does it Cause Apoptosis?

Faezeh Nemati Karimooy ${ }^{1}$, Alireza Ebrahimzadeh Bideskan', Abbas Mohammadi Pour ${ }^{2}$, Seyed Mahmoud Hoseini ${ }^{3}$
${ }^{1}$ Binaloud Healthcare Network, Mashhad University of Medical Sciences, Mashhad, Iran

${ }^{2}$ Department of Anatomy and Cellular Biology, Faculty of Medicine, Mashhad University of Medical Sciences, Mashhad, Iran

${ }^{3}$ Department of Physiology, Faculty of Medicine, Mashhad University of Medical Sciences, Mashhad, Iran

The Neuroscience Journal of Shefaye Khatam, 2019; 7(3): P170

Introduction: Stanozolol is the most appealing drug of Anabolic-Androgenic Steroids that is abused by athletes in high doses for improving their energy, appearance and physical size. Being proved before, Stanozolol causes changes in behaviour and has various physical effects. Studies have been conducted on its neurotoxic impacts on CNS that are mostly psychological-based. Current study was performed to investigate the apoptotic effect of Stanozolol on different parts of rat's hippocampus. Materials and Methods: Sixteen male Wistar rats were divided randomly and equally into two groups (control and experimental). The experimental group received subcutaneous injections of Stanozolol $(5 \mathrm{mg} / \mathrm{Kg} /$ day $)$ for 28 days uninterruptedly. The control group was treated with normal saline in the same manner. After routine procedures, the coronal sections of rats' brains were stained with Toluidine blue and TUNEL for pre-apoptotic and apoptotic cell detection respectively. In order to compare the groups, the mean number of TUNELpositive and pre-apoptotic neurons per unit area were calculated and analyzed. Results: Our histopathological examination revealed the mean number of pre-apoptotic and apoptotic neurons in the CA1, CA2, CA3 and DG area of hippocampus have significantly increased in Stanozolol group. Conclusion: It can be said that abusing Stanozolol may induce pre-apoptotic and apoptotic cell formation in different regions of hippocampus.

\section{P171}

Effect of Aspirin and Conditioned Medium Derived Adipose Mesenchymal Stem Cells co-Administration on Myelin Repair in Lysolecithin-Induced Demyelination Model

Adel Galeshi', Maryam Ghasemi Kasman', Farideh Fayzi ${ }^{2,3}$

${ }^{1}$ Student Research Committee, Babol University of Medical Sciences, Babol, Iran

${ }^{2}$ Cellular and Molecular Biology Research Center, Health Research Center, Babol University of Medical Sciences, Babol, Iran

${ }^{3}$ Department of Anatomical Sciences, Faculty of Medicine, Babol, Iran

The Neuroscience Journal of Shefaye Khatam, 2019; 7(3): P171

Introduction: Several lines of evidence showed the beneficial impacts of aspirin and mesenchymal stem 
cell (mscs) on myelin repair. In the preset study, coapplication of aspirin and conditioned media from Mscs on remyelination has been evaluated in lysolecithin (LPC)-induced demyenation of optic chiasm. Materials and Methods: All expremental procedure were carried out adult male wistar rats. LPC $(1 \%, 2 \mu \mathrm{l})$ was injected into the optic chiasm to induce. The local demyelination model. Animals underwent daily injection of conditioned medium (CM) and oral gavage of aspirin at dose of $25 \mathrm{mg} / \mathrm{kg}$. The conductivity visual signals was assessed using visual evoked potential recordings (VEP) before LPC injection and also on days7 and 14 post lesion. Results: Our results showed that application of CM and aspirin could significantly reduce the latency of VEP waves in LPC receiving animals. Conclusion: The number of oligodendrocyte precursor cells ( opcs) was also significantly increased in optic chiasm of overall, the results of this study suggest that co-administration of aspirin and $\mathrm{CM}$ enhance the myelin repair of optic chiasm in LPC- induced.

\section{P172}

Evaluation of Vitamin E Effects on Behavioral, Motion and Cognitive Function in Autistic Model Rats

Mehrnoush Rahmani, Fatemeh Rezamohammadi, Abbas Ali Vafaei, Katayoun Sedaghat, Abbas Ali Taherian, Ali Rahidypour

Student Research Committee, Department of Research Center and Physiology, Faculty of Medicine, Semnan University of Medical Sciences, Semnan, Iran

The Neuroscience Journal of Shefaye Khatam, 2019; 7(3): P172

Introduction: Previous studies have shown that autism, is a disorder of the advanced neurodegenerative group that is characterized by disturbances in social and communication relationships, often associated with repetitive and stereotypical behaviors and patterns. Some studies have suggested that vitamin E may have a beneficial effect on autism-related disorders. The aim of this study was to determine the effect of this vitamin administration on behavioral, motor and cognitive functions in autistic model rats. Materials and Methods: In this experimental study, valproic acid was injected on day 12 of pregnancy (when the embryonic development of the neural tube occurs) for induction of autism. Also, some groups were injected with Vitamin E (5 and 10 micrograms per kilogram of animal weight) or vehicle were placed intraperitoneally. On the other hand, all mice were tested on days 30 and 60 (Maze Plus, Hot plate, Rotarod, Open field, Morris Water Maze). Results: Data analysis showed that injection of valproic acid can cause behavioral disorders including decreased social interactions, increased repetitive behaviors, increased pain tolerance thresholds, increased anxiety, decreased behavioral behaviors, reduced balance power and motor learning, and cognitive change in rat infants undergo postnatal evaluation, which is usually done on days 30 and 60, and the administration of Vitamin E can significantly modify these abnormalities. $(\mathrm{P}<0.05)$. Conclusion: These findings showed that Vitamin $\mathrm{E}$ can modify behavioral, motor and cognitive functions in autistic model mice and may be effective in the pharmacological treatment of autistic dysfunction.

\section{P173}

Maternal Exposure to Atrazine Induces Hippocampus Neuroinflammation in Mice Offspring and Impairs Their Learning and Spatial Memory

\section{Hamidreza Rastegar Moghaddam ${ }^{1}$, Abbas Mohammadipour ${ }^{1}$, Mahmoud Hosseini, ${ }^{3}$ Alireza Ebrahimzadeh-Bideskan ${ }^{1}$,}

'Department of Anatomy and Cell Biology, Faculty of Medicine, Mashhad University of Medical Sciences, Mashhad, Iran

${ }^{2}$ Microanatomy Research Center, Faculty of Medicine, Mashhad University of Medical Sciences, Mashhad, Iran

${ }^{3}$ Psychiatry and Behavioral Research Center, Department of Neurocognitive Sciences, Mashhad University of Medical Sciences, Mashhad, Iran

The Neuroscience Journal of Shefaye Khatam, 2019; 7(3): P173

Introduction: Atrazine as an herbicide is widely used. Atrazine can be transferred from mother to their offspring via the placenta and the breast milk. Entrance of atrazine to the body may produce neuroinflammation in which effect on learning and memory. In this regard, we evaluated the effects of atrazine exposure during pregnancy and lactation on spatial memory and learning in mice offspring. Materials and Methods: 24 Pregnant $\mathrm{Balb} / \mathrm{C}$ mice were divided into three groups including atrazine $10 \mathrm{mg} / \mathrm{kg}$, atrazine $50 \mathrm{mg} / \mathrm{kg}$ and normal saline $(n=8)$. Daily administration of atrazine or normal saline began from gestational day (GD) 6 and continued until postnatal day (PD) 23 via gavage. At the end of the administration, from each mother two male offspring were selected randomly and then their brain were removed, fixed in formalin and prepared according to routine histological methods. The tissue sections stained with TUNEL and Toluidine blue to detect apoptotic cells and dark neurons in their hippocampus respectively. Finally, the stained slides were photographed and apoptotic cells and dark neurons were counted per unit area in the CA1, CA2, CA3 and DG hippocampus regions by using the soteriological methods. Moreover, remaining offspring were weaned and housed until adulthood (PD75) and then two male offspring from each mother were selected randomly for learning and spatial memory assessment by using Morris water maze (MWM) and passive avoidance (PA) tests. Results: The results of this study showed that apoptotic cells and dark 
neurons were significantly increased in CA1 and CA3 regions of the mice offspring hippocampus in atrazineexposed groups comparing to normal saline group $(p<0.05)$. Furthermore, the results of the behavioral tests indicated that learning and memory of offspring in the atrazine groups was significantly lower than normal saline group $(p<0.05)$. Conclusion: impairment of memory and learning in behavioral tests and also the significant alteration of apoptotic cells and dark neurons in the hippocampus of atrazine groups, may be resulted from inflammation in the brain of male offspring.

\section{P174}

The Protective Activity of Nanomicelle Curcumin in Bisphenol A-Induced Neurotoxicity Following Subacute Exposure in Rats

\section{Mahmoud Gorji Valokola ${ }^{1}$, Somayeh Nazari ${ }^{1}$, Mohsen Imenshahidi ${ }^{1,2}$}

${ }^{1}$ Department of Pharmacodynamics and Toxicology, Faculty of Pharmacy, Mashhad University of Medical Sciences, Mashhad, Iran

${ }^{2}$ Pharmaceutical Research Center, Pharmaceutical Technology Institute, Mashhad University of Medical Sciences, Mashhad, Iran

The Neuroscience Journal of Shefaye Khatam, 2019; 7(3): P174

Introduction: Bisphenol A (BPA), a nonsteroid endocrine-disrupting chemical (EDCs) and a xenoestrogen, is used in production of polycarbonate plastics and epoxy resins. Curcumin, a bright yellow pigment and the active component of turmeric, is a strong protective compound against brain disorders. The purpose of the study the protective property of nanomicelle curcumin on BPA induced sub-chronic neurotoxicity in rats was assessed. Materials and Methods: Rats were divided into six groups including control ,BPA ०•) $\mathrm{mg} / \mathrm{kg}$,gavage ,(nanomicelle curcumin $\bullet \cdot$ ) $\mathrm{mg} / \mathrm{kg}$, gavage, (nanomicelle curcumin $(10,25$, and $50 \mathrm{mg} / \mathrm{kg})$ along with BPA $50 \mathrm{mg} /$ $\mathrm{kg}$. The treatments were continued for 4 weeks. Biochemical analysis (SOD, CAT, GST, GPx and GR), measurement of MDA concentration, and GSH content were evaluated. Results: Results displayed that BPA significantly increased malondialdehyde concentration ,SOD and GST enzymes activity and decreased glutathione content ,CAT ,GPx and GR enzymes activity in comparison with control group .However ,nanomicelle curcumin ०•) $\mathrm{mg} / \mathrm{kg}($ significantly improved these toxic effects of BPA in rat cerebellum, cortex ,and hippocampus tissues. Conclusion: The results of this study support the idea that nanomicelle curcumin exhibited preventive effects on sub-chronic exposure to BPA induced neurotoxicity in the brain tissue in rats.

\section{A Girl with Regression and Chorea: A Case Report \\ Farnoosh Ebrahimzadeh, Mehran Beiraghi Toosi \\ Department of Internal Medicine, Faculty of Medicine, Mashhad University of Medical Sciences, Mashhad, Iran}

The Neuroscience Journal of Shefaye Khatam, 2019; 7(3): P175

We report an 8-year old girl with progressive movement disorder and worsening in school performances. Her grandfather was lost in 55 years old and her father had early dementia and lost in 32 years old. She was the first child of twin delivery without any problem and was good up to 6 years old. Her sister (the second twin) had developmental delay and worsened from 6 years old. In MRI she had only mild atrophy in head of caudate nucleus. According to history and physical examination we checked for Huntington disease that was strongly positive. Now in 13 years old, she and her sister are alive and under treatment of drugs and botulinum toxin. Huntington disease is a rare progressive autosomal dominant brain disorder ( 3 to 7 per 100,000 people) that causes abnormal movements, emotional problems, and loss of cognition. The most common form of Huntington disorder is adult form that starts in thirties or forties. In this form, early signs and symptoms include mainly irritability and depression. They may have mild abnormal movements, poor coordination, and also trouble in learning new information. The juvenile form begins in childhood or adolescence and is less common form. They mainly have movement disorder and sometimes mental and emotional problems and decline in school performance. Juvenile Huntington disease tends to progress more quickly than the adultonset form; affected individuals usually live 10 to 15 years after signs and symptoms appear. Normally, the CAG segment (nucleotides) is repeated 10 to 35 times within the gene. In people with Huntington disease, the CAG segment is repeated 36 to more than 120 times. Patients with the adult-onset form, typically have 40 to 50 CAG repeats in the HTT gene, while children with the juvenile form, tend to have more than $60 \mathrm{CAG}$ repeats (anticipation phenomenon).

\section{P176}

The Effect of Combined Dose of Estrogen and Progesterone on the Expression of TNF-A and IL-1及 Inflammatory Cytokines and The Rate of Migration of Lymphocytes to The Ischemic Margin After the Creation of Stroke Model in Rat

Parvin Lotfi, Homayon Naderian, Mahdi Rafiyn, Mohammadali Atlasi, Abolfazl Azami

Anatomical Research Science Center, Kashan University of Medical Sciences, Kashan, Iran

The Neuroscience Journal of Shefaye Khatam, 2019; 7(3): P176 
Introduction: Stroke is a major cause of death and long-term disability due to cerebrovascular obstruction which triggers inflammation in the damaged area of the brain. Although many attempts have been made to treat stroke an effective treatment hasn't been found. Based on neuroprotective effect of estrogen and progesterone hormones, in this study the effect of their combined dose on the expression of TNF- $\alpha$ and IL- $1 \beta$ inflammatory cytokines and the rate of CD4+ cells in the ischemic margin after the creation of MCAO was investigated. Materials and Methods: 27 Wistar male rats were studied by dividing into three groups: ischemia, hormone and sham (control group). the middle cerebral artery was closed for one hour and immediately a combination dose of estrogen and progesterone was injected to the animal. The volume of infarct area was measured by TTC staining and the rate of migration of lymphocytes to the pneumobra area was investigated using IHC techniques and expression of TNF- $\alpha$ and IL- $1 \beta$ genes using the realtime PCR technique. The result were evaluated using ANOVA and Tukey test. Results: Injection of combined dose of estrogen $(25 \mu \mathrm{g} / \mathrm{kg})$ and progesterone $(10 \mathrm{mg} /$ $\mathrm{kg}$ ) Immediately after focal ischemia significantly decreased the area of the infarct, the expression of TNF- $\alpha$ and IL- $1 \beta$ genes, CD4+ cells in the ischemic margin and as a result, inflammation has been reduced. also it significantly increased the behavioral test score. Conclusion: The results showed that the combined dose of estrogen and progesterone hormones had antiinflammatory effect and reduced CD4+ cells in ischemic margin after focal ischemia.

\section{P177}

The Role of Wnt/ $\beta$-Catenin Signaling Pathway in Early Dopaminergic Differentiation of Trabecular Meshwork-Derived Mesenchymal Stem Cells

Faezeh Sahebdel', Ehsan Arefian', Azita Parvaneh Tafreshi ${ }^{3}$, Bahman Zeynali ${ }^{1}$

${ }^{1}$ Faculty of Biology Sciences, University of Tehran, Tehran, Iran ${ }^{2}$ Department of Microbiology, Faculty of Biology Sciences, University of Tehran, Tehran, Iran

${ }^{3}$ National Research Institute of Genetic Engineering and Biotechnology, Department of Basic Sciences, Tehran, Iran

The Neuroscience Journal of Shefaye Khatam, 2019; 7(3): P177

Introduction: Parkinson's disease is the second most common neurodegenerative disorder. Degeneration of dopaminergic neurons in the Substansia nigra causes the loss of dopaminergic function. It has been shown that Wnt/ $\beta$-catenin signaling pathway is involved in differentiation of dopaminergic neurons. Our preliminary study showed that the neural crest-derived trabecular meshwork MSCs (TM-MSCs) could be an appropriate source of stem cells to differentiate into neurons. Therefore, the aim of this study was to examine neural differentiation potential of these cells compared to two other sources of MSCs including adiposederived and bone marrow MSCs and to determine the role of $\mathrm{Wnt} / \beta$-catenin signaling pathway in early dopaminergic differentiation. Materials and Methods: To address these questions, the cells were cultured in the presence and absence of neural induction medium for six days and were analyzed by Real time PCR and immunofluorescence staining. Results: Morphometric analysis showed that TM-MSCs exhibited a better neural characteristic morphology than the other two sources of cells. Real time PCR studies revealed the expression of neural markers, Nurr1 and Map2, were upregulated in neural induced TM-MSCs compared to cells cultured at the absence of it. Plus, we studied the expression of Nurr1 in TM-MSCs after dopaminergic differentiation by immunofluorescence staining, and it was upregulated in treated samples. CHIR was used to induce $\mathrm{Wnt} / \beta$ catenin signaling pathway. In the presence of both CHIR $(3 \mu \mathrm{M})$ and neural induction medium, the expression of neural markers was increased compared to cells cultured only at the presence of neural induction medium. Expressions of $\mathrm{Wnt} / \beta$-catenin signaling target genes, c-Myc and CyclinD1, were increased in CHIR treated TM-MSCs, indicating the activation of $\mathrm{Wnt} / \beta$-catenin signaling in these cells. Conclusion: Our results suggest that TM-MSCs are a better candidate for dopaminergic differentiation than the other commonly used MSCs and Wnt/ $\beta$-catenin Signaling pathway has an important role during this differentiation.

\section{P178}

The Relationship Between Spiritual Health and Mental Health in Women with Multiple Sclerosis of Arak in 1397

\section{Shadi Moghaddam ${ }^{1}$, Sepideh Khanlari' ${ }^{2}$ Fatemeh Ranjbar ${ }^{2}$}

${ }^{1}$ Department of Nursing, Faculty of Medical Sciences, Arak Branch, Islamic Azad University, Arak, Iran

${ }^{2}$ Student Research Committee, Department of Health, Arak Branch, Islamic Azad University, Arak, Iran

The Neuroscience Journal of Shefaye Khatam, 2019; 7(3): P178

Introduction: The lack of spiritual health, especially in chronic diseases, provides many psychological problems and physical complications. Since spirituality plays an important role in mental health, it can be an effective source of adaptation to stressful events. nowadays, the number of people who believe that spirituality is the way to treat their spiritual and spiritual disorders. Chronic diseases and its treatments have a negative effect on the various physical, psychological and social aspects of an individual's life. Multiple sclerosis is the most common cause of progressive neurodegenerative diseases; which women are 2 to 3 times more likely than men with the 
disease. The purpose of this study was to determine the relationship between mental health and mental health in women suffering from multiple sclerosis in Arak city in 1397. Materials and Methods: In this descriptive cross-sectional correlation study, 290 female MS patients who were in advanced stage of the disease were referred to Valiasr Hospital in Arak, they were selected randomly in a proportional volume using Cochran formula in 1397. The instruments used in the research were personal information questionnaire, GHQ-28 general mental health questionnaire, and PulootzinEllison's 20-item questionnaire on spiritual well-being. After collecting data, the data were analyzed by SPSS16 statistical software, correlation and regression tests. Results: The results of this study showed that there is a significant relationship between mental health and its dimensions with mental health $(p<0.01)$. As with increasing spiritual well-being, the level of depression and anxiety decreased in patients $(\mathrm{p}<0.05)$. There is a significant relationship between level of education, marital status and mental health in patients $(p<0.05)$. Conclusion: Considering the positive and significant role of spiritual health in mental health, by promoting the spiritual dimension, mental health of women with multiple sclerosis can be improved.

\section{P179}

Assessment the Activity of HTLV-1 in HAM/TSP Patients Using Tax mRNA Expression and Proviral Load After in Vitro Lymphocyte Activation

Sanaz Ahmadi Ghezeldasht, ${ }^{1}$, Atefeh Yari ${ }^{3}$, Arman Mosavat ${ }^{1}$, Seyyed Abdolrahim Rezaee ${ }^{2}$

${ }^{1}$ Blood Borne Infections Research Center, Academic Center for Education, Culture and Research (ACECR), Mashhad, Iran ${ }^{2}$ Immunology Research Center, Department of Inflammation and Inflammatory Diseases, Mashhad University of Medical Sciences, Mashhad, Iran

${ }^{3}$ Department of Virology, Faculty of Public Health, Tehran University of Medical Sciences, Tehran, Iran

The Neuroscience Journal of Shefaye Khatam, 2019; 7(3): P179

Introduction: HTLV-1 is the first human retrovirus recognized and associated with the HAM / TSP and ATLL. Studies have shown that that approximately ninety-five percent of carriers infected with HTLV-1 remain asymptomatic and less than five percent develop an inflammatory neurologic disease, mainly HTLV-1 Associated Myelopathy/Tropical Spastic Paraparesis (HAM / TSP). Therefore, proviral loading with Tax may affect cellular genes such as cytokines and oncogenes, while being implicated in pathogenicity. Materials and Methods: To measure HTLV-1 activity, thirty HAM/ TSP patients, thirty HTLV-1 healthy carriers, and MT-2 cell line were evaluated. PBMCs were isolated and activated using PMA and ionomycine. Real-time PCR and TaqMan methods were carried out using specific primers and fluorescence probes for Tax expression and proviral load assessment. B2microglobulin ( $\beta 2 \mathrm{~m})$ and albumin were used as controls in Tax expression and in proviral load, respectively. Results: An insignificant increase in Tax expression was observed in HAM/TSP patients compared to healthy carriers. However, after lymphocyte activation there was a significant increase in Tax expression in HAM/TSP patients $(\mathrm{P}=0.042)$. The Proviral load in patients was significantly higher than in carriers. Moreover, there was a significant correlation between Tax mRNA expression in activated PBMCs and proviral load $(\mathrm{R}=0.37, \mathrm{P}=0.012)$. Conclusion: Even if proviral load was considered as a valuable index for monitoring HTLV-1 infected individuals, the results of this study demonstrated that Tax expression in activated PBMCs along with proviral load assessment in HAM/ TSP patients are a more reliable factor for determining the prognosis and monitoring healthy carriers and HAM/ TSP patients.

\section{P180}

The Effect of Ghrelin Intrahippocampal Injection on Motor Balance, Memory in Rat

\section{Vahideh Sahraiian, Homayoun Khazali}

Faculty of Biological Sciences, Shahid Beheshti University, Tehran, Iran

The Neuroscience Journal of Shefaye Khatam, 2019; 7(3): P180

Introduction: Today, memory impairment in neurodegenerative diseases Like Alzheimer, is one of the major dilemmas. Ghrelin is a peptide hormone that is synthesized by hypothalamus, pituitary, and some other tissues. This hormone may affect learning and especially motion memory in the hippocampus. The aim of this study was to investigate the effects of ghrelin intrahippocampal injection otor coordination, memory. Materials and Methods: 36 male rats were divided into 6 groups, including control, saline, ghrelin $(0.3$, 1.5 or $3 \mathrm{nmol}$ ) and citicoline. All groups were trained in the rotarod test for 3 times to each rat. Results: the result showed that dosage $3 \mathrm{nmol}$ of ghrelin significantly can be improved motor coordination and memory in competition to citycholine $(\mathrm{P}<0.0001)$. Conclusion: It seems that ghrelin could be used to treat memory disorders such as Alzheimer's

\section{P181}

Effects of Berberine on Glutamate-Induced Toxicity in PC12 and N2a Cells

Fatemeh Forouzanfar', Hamid Reza Sadeghnia ${ }^{2}$, Hossein Hosseinzadeh ${ }^{3}$ Monireh Kolangikhah ${ }^{1}$, Elham Asadpour ${ }^{4}$

${ }^{1}$ Department of Neuroscience, Faculty of Medicine, Mashhad 
University of Medical Sciences, Mashhad, Iran

${ }^{2}$ Pharmacological Research Center of Medicinal Plants, Mashhad University of Medical Sciences, Mashhad, Iran

${ }^{3}$ Department of Pharmacodynamics and Toxicology, Faculty of Pharmacy, Mashhad University of Medical Sciences, Mashhad, Iran

${ }^{4}$ Anesthesiology and Critical Care Research Center, Shiraz University of Medical Sciences, Shiraz, Iran

The Neuroscience Journal of Shefaye Khatam, 2019; 7(3): P181

Introduction: Glutamate is the main excitatory neurotrans-mitter in the brain. Neurodegenerative diseases have been associated with glutamatergic dysfunction. Berberine, an isoquinoline alkaloid broadly present in different medicinal herbs, has been reported to have neuroprotective effect. In the present study, the effects of berberine against glutamate-induced oxidative damage and apoptosis were investigated. Materials and Methods: The cultured PC12 and N2a cells were pretreated $(2 \mathrm{hr})$ with varying concentrations of berberine (50-1000 $\mu \mathrm{M})$, followed by exposure to glutamate (10 $\mathrm{mM}$ ) for $24 \mathrm{hr}$. The cells viability, intracellular reactive oxygen species (ROS), lipid peroxidation, glutathione (GSH) content, superoxide dismutase (SOD) activity, DNA fragmentation and the expressions of pro-apoptotic (cleaved caspase-3 and bax) and anti-apoptotic (bcl2) proteins were then measured. Results: In both cell lines, pretreatment with berberine (especially at low concentrations) significantly decreased ROS generation, lipid peroxidation, and DNA fragmentation, while improving glutathione content and SOD activity in glutamate-injured cells. Moreover, berberine showed anti-apoptotic effects by reducing the glutamate-evoked caspase-3 and bax/bcl-2 overexpression. Conclusion: The results of present study suggest that berberine protects against glutamate-induced $\mathrm{PC} 12$ and $\mathrm{N} 2$ a cells injury by decreasing oxidative stress and subsequently inhibiting apoptosis. This is relevant to berberine treatment in neurodegenerative disorders, such as dementia (Alzheimer's disease), seizures, and stroke.

\section{P182}

Effect of Recombinant Human Erythropoietin on Zinc-Induced Traumatic Brain Injury in Rats

\section{Zahra Poustchian}

Department of Neuroscience, Mashhad University of Medical Sciences, Mashhad, Iran

The Neuroscience Journal of Shefaye Khatam, 2019; 7(3): P182

Introduction: Traumatic Brain Injury (TBI) is one cause of neuronal death that results in losing memory, anxiety, and depression. Researches indicate that zinc contributes to transmission in synapses in the central nervous system(CNS) but is released in toxic level from neurons in TBI synaptically. Releasing zinc from the presynaptic neuron in TBI aggravates inflammation in brain. The aim of this study is assessing the effect of Recombinant Human Erythropoietin (RhEpo) on neuronal survival after TBI. In this present case-control study, 30 male Sprague-Dawley rats with weight 250300 gr were divided into three groups: RhEpo, control, and sham. Hippocampal neurons of rats in control and RhEpo groups were cultured and exposure to $200 \mu \mathrm{M}$ $\mathrm{ZnCl} 2$ in 20 minutes. This procedure caused neuronal injury that was revealed by observing nerve morphology. Mitochondrial function measurement showed that 24 hours before zinc exposure, using RhEpo resulted in a remarkable increase of neuron survival in RhEpo group (0.6007) vs in the control group $(0.2333, p<0.01)$. Also study showed that application of RhEpo 30 minutes after TBI stopped neuronal death which was revealed by ZP2, a zinc-specific fluorescent sensor to show neuronal damage. However mechanism of RhEpo function in TBI is not clear, data's show a significant effect of RhEpo on decreasing neuronal death $(p<0.01)$. These results demonstrate RhEpo can dramatically reduce $\mathrm{Zn} 2+$ releasing in the hippocampus after TBI. Understanding optimal RhEpo and zinc serum level in persons with TBI can help to treatment strategies.

\section{P183}

Early Intervention in Inflammatory Processes During Febrile Seizures, Prevent Subsequent Memory Deficits in Wistar Rat Nosaibeh Riahi Zaniani', Ali Moghimi', Ali
Roohbakhsh

${ }^{1}$ Rayan Center for Neuroscience and Behavior, Deptartment of Biology, Ferdowsi University of Mashhad, Mashhad, Iran ${ }^{2}$ Pharmaceutical Research Center, Pharmaceutical Technology Institute, Mashhad University of Medical Sciences, Mashhad, Iran

The Neuroscience Journal of Shefaye Khatam, 2019; 7(3): P183

Introduction: Febrile seizures are the most common type of seizures that $2-14 \%$ of infants and preschool children suffering from it in the world. Increasing evidence indicates close relation between Epileptogenesis, pathogenesis of seizures and inflammatory processes. Based on associated studies, some of the inflammatory mediators such as prostaglandins, cytokines and complement factors, play a more noted role in promoting inflammation and seizure. These mediators of inflammation apply detrimental effects on learning and memory. It seems plausible that interfering with these molecules or their receptors can affect seizures consequences. We intend to determine the relationship between Toll-like receptor 4 antagonizing in rat pups with febrile seizure and amelioration of memory deficits in adulthood. Materials and Methods: Complex febrile seizure was induced for three Consecutive sessions 
during p9-p11 in wistar male rats by a heated chamber. The animals were divided into 7 groups ( $\mathrm{n}=16$ for each): Control (completely intact), Sham (hyperthermia that received Dimethyl sulfoxide as a solvent). 3rd group was hyperthermia without any injections. In other 3 experimental groups (treatments) different doses of TLR4 receptor antagonist (TAK-242) was injected via IP route. 7 th group was positive control (pups received the effective dose of antagonist without hyperthermia). Prominent neuroinflammatory factors and receptor (levels of IL-1 $\beta$, TNF- $\alpha$ and TLR4, respectively) were measured by immunohistochemistry and western blot in ratpup's hippocampi.Also P11 rats were exposed to videoEEG monitoring and after 50 days' adult rat exposed to the Novel Object Recognition Task to test learning and memory. Results: Evaluation of the ictogenic cytokines IL-1 $\beta$ and TNF- $\alpha$ also TLR4 expression post febrile seizure induction proved inflammation progression in the brain of sham and hyperthermic groups. The results in treatment groups showed that TAK-242 injections dose-dependently significant amelioration of inflammatory factors. Behavior of adult rats in NORT showed displayed cognitive impairment in sham and patient groups $(\mathrm{P}<0.05)$ while the rat's performance in treatment with TLR4 antagonist showed significant improvement $(\mathrm{P}<0.01)$. Conclusion: It is suggested that TLR4 antagonist can be exploited not only as a Preventative barrier for seizure progression but also, could be considered as reliable route to improve febrile seizure consequences like memory deficits.

\section{P184}

Does IL-18 Promoter Gene Polymorphisms Increase Risk of Multiple Sclerosis?

Sadra Ansaripour ${ }^{1}$, Niloofar Safaei ${ }^{1}$, Niloofar Nezaminia ${ }^{1}$, Parna Alikhani ${ }^{1}$, Najme Bagheri ${ }^{1}$, Nahid Jivad $^{2}$

${ }^{1}$ Student Research Committee, Shahrekord University of Medical Sciences, Shahrekord, Iran

${ }^{2}$ Department of Neurology, Faculty of Medicine, Shahrekord University of Medical Sciences, Shahrekord, Iran

The Neuroscience Journal of Shefaye Khatam, 2019; 7(3): P184

Introduction: Multiple sclerosis(MS) is an inflammatory and degenerative disease of the central myelin, which can lead to a significant disability. The aim of this study was to investigate IL-18 gene polymorphisms in multiple sclerosis patients referring to Shahrekord health centers.

Materials and Methods: This case-control study was carried out on 57 relapsing remitting multiple sclerosis (RRMS) patients and 57 healthy individuals as control group in Shahrekord university of medical sciences. Blood samples were collected and DNA was extracted by salting out method. The alleles of each of the IL-18 genes polymorphisms were determined using a specific two-stage allele polymerase chain reaction. All PCR reactions were carried out in a mix volume of $25 \mu \mathrm{l}$. PCR was performed for rs187238 and rs1946518 and the PCR products were analyzed on $2 \%$ agarose gel stained with ethidium bromide. Collected data were analyzed by SPSS software. Results: Among the studied patients, $69.3 \%$ were female and $30.7 \%$ were male. The frequency of $\mathrm{GG}, \mathrm{CC}$, and $\mathrm{GC}$ genotypes for IL-18137 gene was $61.4 \%, 12.3 \%$ and $26.3 \%$, respectively, in $\mathrm{MS}$ patients versus $54.4 \%, 8.8 \%$ and $36.8 \%$ in the control group $(\mathrm{P}>0.05)$. Frequency of $\mathrm{AA}, \mathrm{AC}$ and $\mathrm{CC}$ genotypes for IL-18-607 gene was $21.1 \%, 38.6 \%$ and $40.4 \%$ in patients with MS and $17.5 \%, 38.6 \%$ and $43.9 \%$ in the control group $(\mathrm{P}>0.05)$. All possible genotypes were detected in the control group, while CC / CC and GC / CC genotypes were not observed in patients with MS. There was no significant difference in the frequency of combined genotypes between patients with MS and control group $(\mathrm{P}>0.05)$. Conclusion: There was no significant difference in distribution of genotypes and alleles of IL18-137 and IL18-607 genes as well as their combined genotypes. Also, similar studies on different ethnic populations can help to clarify the association between IL-18 polymorphisms and MS disease.

\section{P185}

The Effects of PPAR- $\gamma$ Agonist Pioglitazone on Hippocampal Cytokines, Brain-Derived Neurotrophic Factor, Memory Impairment, and Oxidative Stress Status in LipopolysaccharideTreated Rats

Farimah Beheshti, ${ }^{1,}$, Mahmoud Hosseini', Milad

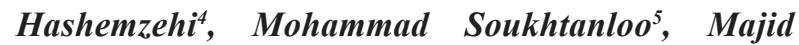
Khazaei $^{6}$, Mohammad Naser Shafei ${ }^{6}$

${ }^{1}$ Neuroscience Research Center, Department of Basic Sciences, Torbat Heydariyeh University of Medical Sciences, Torbat Heydariyeh, Iran

${ }^{2}$ Department of Physiology, Faculty of Paramedical Sciences, Torbat Heydariyeh University of Medical Sciences, Torbat Heydariyeh, Iran

${ }^{3}$ Psychiatry and Behavioral Sciences Research Center, Department of Neurocognitive Sciences, Mashhad University of Medical Sciences, Mashhad, Iran

${ }^{4}$ Iranshahr University of Medical Sciences, Iranshahr, Iran ${ }^{5}$ Department of Biochemistry, Faculty of Medicine, Mashhad University of Medical Sciences, Mashhad, Iran

${ }^{6}$ Neurogenic Inflammation Research Center, Faculty of Medicine, Mashhad University of Medical Sciences, Mashhad, Iran

The Neuroscience Journal of Shefaye Khatam, 2019; 7(3): P185

Introduction: In the present study, the effects of pioglitazone on hippocampal cytokines, brain-derived neurotrophic factor (BDNF), oxidative stress status and memory in lipopolysaccharide (LPS) treated rats were investigated. Materials and Methods: The rats were 
divided into five groups ( $\mathrm{n}=10$ in each) :(1) control, (2) LPS ( $1 \mathrm{mg} / \mathrm{kg}$, two hours before behavioral tests), (3-5) LPS-Pio $10 \mathrm{mg}$, LPS-Pio $20 \mathrm{mg}$ and LPS-Pio $30 \mathrm{mg}$ groups $(10,20$ or $30 \mathrm{mg} / \mathrm{kg}$ pio $30 \mathrm{~min}$ before LPS). Finally, we removed brain tissue for biochemical measurements. Results: In LPS group, the escape latency in Morris water maze test was longer while, the delay timeto enter the dark compartment in passive avoidance test were significantly shorter than in the control. Treatment by 20 and $30 \mathrm{mg}$ of pioglitazone corrected these parameters. Malondialdehyde (MDA), nitric oxide metabolites, interleukin-6 (IL-6) and tumor necrosis factor- $\alpha$ (TNF $\alpha$ ) concentration in the hippocampus of LPS group were higher than control group while, in LPS-Pio20mg an LPS-Pio30mg group they were lower than LPS group. The thiol content, IL10 concentration and the activities of catalase (CAT) and superoxide dismutase (SOD) in hippocampal tissues of LPS group reduced compared to control group. These factors enhanced in LPS-Pio20 mg and LPS-Pio30 mg groups compared to LPS. The hippocampal content of BDNF in LPS group was significantly lower compared to the control group. All treated groups had higher BDNF content in comparison to LPS group. Conclusion: The findings indicated that protective effects of pioglitazone against LPS-induced memory impairment were accompanied by decreasing of inflammatory cytokines, oxidative stress and increasing of BDNF.

\section{P186}

Diagnosis of Epilepsy by Using of Electroencephalogram and Estimation of Higher Order Spectrum

\section{Maryam Naghavizadeh, Seyed Amirhosein Mousavi}

Department of Biomedical Engineering, Faculty of Engineering, Mashhad Branch, Islamic Azad University, Mashhad, Iran

The Neuroscience Journal of Shefaye Khatam, 2019; 7(3): P186

Epilepsy is not a specific disease; it is a brain disorder caused by neuronal activity in the brain. There are six types of common epileptic seizures, including epilepsy with seizure and fainting, tonic, tonic epilepsy, with seizure and fainting, muscle tension, distress and weak attacks. All attacks cause loss of consciousness. Tensileelastic attacks accompanied by shrinkage of the organs and then their elongation, and the waist simultaneously, which lasts 10 to 30 seconds, is a tensile phase. A cry is heard from the contraction of the chest muscles. In this study, we used an estimate of the high-order spectrum and extracted characteristics to obtain an automatic classification between seizure and non-seizure state with a precision of $99.9 \pm 0.05$.

\section{P187}

The Beneficial Effect of Aminoguanidine on Learning and Memory Impairment in lipopolysaccharide Treated Rats

Vajiheh Alikhani, Farimah Beheshti ${ }^{2,3}$, Mahmoud Hosseini, Majid Khazaei ${ }^{4}$, Hamid Reza Sadeghnia ${ }^{5}$, Mohammad Soukhtanloo ${ }^{6}$

${ }^{1}$ Neurogenic Inflammation Research Center, Mashhad University of Medical Sciences, Mashhad, Iran

${ }^{2}$ Neuroscience Research Center, Torbat Heydariyeh University of Medical Sciences, Torbat Heydariyeh, Iran

${ }^{3}$ Department of Physiology, Faculty of Paramedical Sciences, Torbat Heydariyeh University of Medical Sciences, Torbat Heydariyeh, Iran

${ }^{4}$ Psychiatry and Behavioral Sciences Research Center, Department of Neurocognitive Sciences, Mashhad University of Medical Sciences, Mashhad, Iran

${ }^{5}$ Pharmacological Research Center of Medicinal Plants, Mashhad University of Medical Sciences, Mashhad, Iran

${ }^{6}$ Department of Biochemistry, Faculty of Medicine, Mashhad University of Medical Sciences, Mashhad, Iran

The Neuroscience Journal of Shefaye Khatam, 2019; 7(3): P187

Introduction: In the present study, the effect of aminoguanidine (AG) as inducible NO synthase (iNOS) inhibitor on lipopolysaccharide (LPS)-induced learning and memory impairment was evaluated. Materials and Methods: The rats were divided into 5 groups and treated as follow: 1) Control (Saline), 2) LPS (1 mg/ $\mathrm{kg}$ ), 3-5) AG 50, 100 and $150 \mathrm{mg} / \mathrm{kg} 30 \mathrm{~min}$ before LPS injection. The treatment was started 5 weeks before the behavioral experiments and continued during the behavioral tests (LPS injection two $h$ before each behavioral experiment). Results: The escape latency in Morris water maze (MWM) test and the latency to enter the dark compartment in Passive avoidance (PA) test in LPS group were significantly higher than in control $(\mathrm{P}<0.001)$ whereas, in AG100-LPS and AG150-LPS groups they were shorter than LPS group $(\mathrm{P}<0.001)$. Conclusion: It is suggested that LPS induced learning and memory impairments are preventable by aminoguanidine as an iNOS inhibitor.

\section{P188}

Interactive Involvement of Hippocampal cAMP/ PKA and Cyclooxygenase-2 Signaling Pathways in Spatial Learning in the Morris Water Maze

Sahar Fanoudi, Kaveh Tabrizian', Mahmoud Hashemzaei ${ }^{2}$

${ }^{1}$ Department of Pharmacology, Faculty of Medicine, Mashhad University of Medical Sciences, Mashhad, Iran

${ }^{2}$ Department of Pharmacology and Toxicology, Faculty of Pharmacy, Zabol University of Medical Sciences, Zabol, Iran

The Neuroscience Journal of Shefaye Khatam, 2019; 7(3): P188

Introduction: Accumulated evidence shows that 
the cAMP-PKA signaling pathway plays a key role in memory functions. Cyclooxygenase-2, a critical player in neuroinflammation, has been confirmed in the pathogenesis of neurodegenerative diseases. This study is aimed to assess the effect of the interaction of cAMPPKA and cyclooxygenase pathways on spatial memory acquisition in animal models. Materials and Methods: In the present study, the effects of the four-day bilateral intra-hippocampal infusions of $\mathrm{H}-89$ as a protein kinase AII inhibitor $(10 \mu \mathrm{M} /$ side $)$, celecoxib $(0.1 \mathrm{M} /$ side $)$ as a selective cyclooxygenase-2 inhibitor, celecoxib/H-89 and bucladesine $(10 \mu \mathrm{M} /$ side $) /$ celecoxib/H-89 on spatial memory acquisition in the Morris water maze were investigated. Control animals received bilateral intrahippocampal infusions of dimethyl sulfoxide. Rats were trained for 4 days; each day included one block of four trials. Post-training probe trial tests were performed on day five. Results: A bilateral intra-hippocampal infusion of H-89 and celecoxib led to a significant impairment in spatial learning compared to the controls through a notable decrease in escape latency and traveled distance. But, combination treatment of animals with celecoxib/H-89 and bucladesine/celecoxib/H-89 could considerably reverse celecoxib and $\mathrm{H}-89$-induced spatial memory acquisition impairments in the Morris water maze. Conclusion: These results indicate the probable regulatory effects of cAMP/PKA and cyclooxygenase-2 signaling pathways on spatial memory acquisition in the Morris water maze.

\section{P189}

\section{Inclusion Body Myositis; in the Differential Diagnosis of Other Inflammator Muscles Diseases}

\section{Shadi Zamanian, Pourakbar Ebrahim}

Department of Neurology, Mashhad University of Medical Sciences, Mashhad, Iran

The Neuroscience Journal of Shefaye Khatam, 2019; 7(3): P189

Introduction: Incusion body myositis (IBM), a condition characterized by progressive muscle weakness and inclusion bodies visible on muscle biopsy, is the most common type of myopathy in patients over 50 years of age. However, it is not only underdiagnosed but frequently misdiagnosed as polymyositis and hence wrongly treated with steriods. Treatment-resistant polymyositis in patients over 50 years of age is often IBM. Case Presentation: An 58-year-old male, presented with progressive painless weaknesses and atrophy on both forearms, hands and lower extremities for 3 years ago. Two years earlier, he had been independent but now required assistance for most activities of daily living such as transfers from bed to chair, climbing stair and toileting. Family history was negative. In medical history , 6 months later he was treated by corticosteroid but suddenly exacerbated the symptoms and showed an inappropriate response. In Neurological examination he had no sensory abnormalities. Deep tendon reflexes were decreased and force reduced in upper and lower limbs in proximal and distal, especially in flexor digital and quadriceps muscles. CPK and LDH were 2504/380 IU/L and Aldolase :7.7, Anti HTLV and muscle PCR were positive other laboratory findings were within normal limits. EMG revealed fibrillation and myotonic discharge at rest and low amplitude motor unit potential with early recruitment. The result of EMG suggested myopathy with inflammation (Irritable myopathy). Biopsy from the left biceps muscle was done. pathological findings were, severe inflammatory myopathy with RED-RIMMED vacuoles and rare congophilic inclusions associated with some ragged red fibers and endomysial fibrosis as well as adipose tissues replacement compatible with inclusion body myositis. The patient was treated with IVIG, responed to treatment with IVIG was minimal improvemented weakness of upper extremities but weakness of lower limbs remained.

\section{P190}

Chitosan and its Anti-Inflammatory Role in CNS Damage

\section{Pegah Khosravian ${ }^{1}$, Moosa Javdani ${ }^{2}$}

${ }^{1}$ Medical Plants Reserch Center, Basic Health Sciences Institute, Shahrekord University of Medical Sciences, Shahrekord, Iran ${ }^{2}$ Department of Clinical Sciences, Faculty of Veterinary Medicine, Shahrekord University, Shahrekord, Iran

The Neuroscience Journal of Shefaye Khatam, 2019; 7(3): P190

Strategies and mechanisms used in order to protect apoptosis, against neuronal damage, degeneration and/ or dysfunction in the central nervous system (CNS) are called as neuroprotection. The target of neuroprotection is to bound neuronal death or dysfunction after CNS damage in a try to maintain the highest possible honesty of cellular interactions in the brain, thus minimizing disorder to neural role. According to its mechanism, neuroprotection can be classification into some mechanisms such as: anti-inflammatory, antioxidant (scavenger/free radical trapper), apoptosis inhibitor, antiexitotoxic, etc. Chitosan is a linear polysaccharide that consists of $\beta$ - $(1 \rightarrow 4)-2$-amino-d-glucose and $\beta$ - $(1 \rightarrow 4)$ 2 -acetamido-d-glucose units derived from partial deacetylation of chitin. Both Chitooligosaccharides (COS) and chitosan are known to possess a lot of biological activities such as anti-inflammatory activities, antioxidant, antibacterial, immunoenhancing, etc. The first response of a body's immune system to pathogens or irritation is inflammation. Some studies are discovering blinding communications between a number of neurodegenerative disorders and chronic inflammation. The neuroinflammation process plays a substantial activity in the progression and initiation of different neurodegenerative diseases. Recently, several studies have found anti-neuroinflammatory role of 
chitosan and its derivatives. One study reported that high molecular weight water soluble chitosan (WSC) inhibits the production of pro-inflammatory cytokine in human astrocytoma cells activated by interleukin-1 $\beta$ (IL-1 $\beta$ ) and $A \beta$ peptide $25-35$ (A $\beta 25-35)$. Another study focused on the anti-neuroinflammatory effect of chitosan and its derivatives on NT2 neuronal cells. Chitosan employs anti-neuroinflammatory role by inhibits the activation of NF- $\mathrm{NB}$ and upregulation of heat shock protein 70 (Hsp-70). The anti-inflammatory mechanism of Hsp-70 is mediated by the binding of Hsp-70 to NF- $\kappa \mathrm{B}$ and its following inhibition. A pre-treatment with 0.1 and $0.5 \%$ (w/v) chitosan prior to $\mathrm{FeSO} 4$ and $\mathrm{H} 2 \mathrm{O} 2$ exposure has been proved to increase the level of heat shock protein (Hsp-70) to 1.4- and 1.6-times, respectively.

\section{P191}

The Importance of Microglia and Mast Cells in Alzheimer's Disease

Parsa Bathhaee ${ }^{1,2}$, Behzad Ensan 1, 2, Mohammad Bagher Soltanian", 2, Mahshid Safari Ghalezoul, 2, Nima Zafari, ${ }^{1,2}$

${ }^{1}$ Student Research Committee, Faculty of Medicine, Mashhad University of Medical Sciences, Mashhad, Iran

${ }^{2}$ Department of Neurology, Faculty of Medicine, Mashhad University of Medical Sciences, Mashhad, Iran

The Neuroscience Journal of Shefaye Khatam, 2019; 7(3): P191

Introduction: Alzheimer's disease (AD) is the most common neurodegenerative disease of the central nervous system (CNS), results in dementia, memory and cognitive impairment. Main pathological characteristics ofAD are extracellular deposits (senile plaques) offibrillar amyloid b-peptide $(A \beta)$ and intracellular neurofibrillary tangles hyperphosphorylated tau protein, alongside signs of activated Microglia and Astrocytes which is often related to $A \beta$ deposition. Neuroinflammation is the immune reactions jeopardize CNS components. $\mathrm{AD}$ is considered as a neuroinflammatory disease involving immune components of CNS. Microglia plays a macrophage-like role in the CNS immune defense. Microglia are associated with the production and release of pro-inflammatory cytokines, most importantly tumor necrosis factor (TNF)- $\alpha$, IL-6, IL-23, IL-1 $\beta$, IL-12, nitric oxide (NO), and chemokines. TLR4 (Toll-like receptor 4 ) is one of many receptors Microglia uses to detect $A \beta$ deposition. Activated Microglia secretes neurotrophic factors and diminishes the $A \beta$ deposits to protect neurons. Although primary activation of Microglia is beneficial, chronic exposure of TLR4 to A $\beta$ can result in TLR signaling dysfunction and inflammation. Mast cells (MCs) are innate immune system cells, present adjacent to blood vessels of most tissues including CNS. MCs are capable of producing a vast range of mediators including cytokines ((TNF- $\alpha)$, IL-1, 2,3,5,6,7,9,13,16 and 33) chemokines, growth factors and etc. MCs are a key component of allergic and inflammatory reactions by rapid degranulation and then slow release of newly synthesized pro-inflammatory mediators and cytokines. MCs have a close connection with Microglia in inflammatory sites of CNS and they are also capable of detecting $A \beta$ deposition and responding to them through inflammatory mediators. Conclusion: Despite MCs close attachment to Microglia and their important role in inflammation, MCs have been neglected. Further studies on MCs and their relation with Microglia as an inseparable inflammatory unit, may lead to a better understanding of $\mathrm{AD}$ and its progression and innovative therapeutic ways.

\section{P192}

Protective Effects of Crocus Sativus (Saffron) and its Constituents on Neurodegenerative Disorders

\section{Mohammad Reza Khazdair ${ }^{1}$ Mohammad Hossein Boskabady', Mahmoud Hosseini ${ }^{3}$}

${ }^{1}$ Torbat Jam Faculty of Medical Sciences, Torbat Jam, Iran ${ }^{2}$ Neurogenic Inflammation Research Center, Department of Physiology, Faculty of Medicine, Mashhad University of Medical Sciences, Mashhad, Iran

${ }^{3}$ Neurocognitive Research Center, Department of Physiology, Faculty of Medicine, Mashhad University of Medical Sciences, Mashhad, Iran

The Neuroscience Journal of Shefaye Khatam, 2019; 7(3): P192

Introduction: Saffron or Crocus sativus L. (C. sativus) has been widely used traditionally as a medicinal plant to promote human health, especially in Asia. The main components of saffron are crocin, and safranal. The median lethal doses (LD50) of C. sativus are $200 \mathrm{mg} / \mathrm{ml}$ and $20.7 \mathrm{~g} / \mathrm{kg}$ in vitro and in animal studies, respectively. Saffron has been suggested to be effective in the treatment of a wide range of disorders including coronary artery diseases, hypertension, dysmenorrhea learning and memory impairments. In addition, different studies have indicated that saffron has anti-inflammatory, and antiatherosclerotic activities. Anti-tussive effects of stigmas and petals of C. sativus and its components, safranal and crocin have also been demonstrated. The anticonvulsant and anti-Alzheimer properties of saffron extract were shown in human and animal studies. The efficacy of C. sativus in the treatment of mild to moderate depression was also reported in clinical trial. Administration of C. sativus and its constituents increased glutamate and dopamine levels in the brain in a dose-dependent manner. It also interacts with the opioid system to reduce withdrawal syndrome. Therefore, in the present article, the effects of C. Conclusion: sativus and its constituents on the nervous system and the possible underlying mechanisms are reviewed. Our literature review showed that $\mathrm{C}$. sativus and its components can be considered as promising agents in the treatment of nervous system disorders. 


\section{P193}

\section{Investigation the Association of Epilepsy with Autism Spectrum Disorders in Children}

\author{
Zeinab Boostani', Zahra Easy ${ }^{2}$, Sepide Cheraghi ${ }^{1}$ \\ ${ }^{1}$ Student Research Committee, Faculty of Basic Sciences, \\ Mashhad Branch, Islamic Azad University, Mashhad, Iran \\ ${ }^{2}$ Student Research Committee, Faculty of Nursery and Midwifery, \\ Mashhad Branch, Islamic Azad University, Mashhad, Iran
}

The Neuroscience Journal of Shefaye Khatam, 2019; 7(3): P193

Introduction: Epilepsy is a series of chronic medical or long term neurological disorders characterized by an epileptic seizure. About $1 \%$ of world population (65million) is epilepsy and almost $80 \%$ of cases are in developing countries. autism is a type of developmental disorders (of a type of social relationship) characterized by abnormal communication and verbal behaviors. Of every 60 to 70 births in the word one has autism .The results showed that among 190 children with $\mathrm{ASD}$ (autism spectrum disorders) $10.5 \%$ had epileptic seizure and $6.3 \%$ had epilepsy.In two articles abnormal physical activity and hearing impairment in ASD patients with epilepsy before the age of one year have been reported. in another article the rate of autism in epilepsy in people with intellectual disabilities is much higher than in those without intellectual disabilities.also the coexistence of autism and epilepsy diseases depends on genetic and environmental factors. It has been stated in most articles that there is a relationship between epilepsy and autism in children. It is also suggested that diagnostic services and treatment of these patients be presented in a dermatologic and neuropsychiatric environment so that the disease can be detected early in the young and treatments fit into that. Conclusion: However, there is still no definitive conclusion and further studies are needed.

\section{P194}

Combining Nanotechnology and Herbal Drug Delivery in Treatment of Alzheimer's Disease

\section{Elahe Alizadeh}

Faculty of Medicine, Zabol University of Medical Sciences, Zabol, Iran

The Neuroscience Journal of Shefaye Khatam, 2019; 7(3): P194

Introduction: Inflammation is implicated in the diverse neurodegenerative disorder. In Alzheimer disease (AD), beta-amyloid peptides accumulate in the brain that activates microglia, produces cytokines and causes oxidative damage. Drug that control inflammation due to lack of penetration in the blood-brain barrier (BBB), wasting drug and clinical interaction is less effective. Pharmaceutical nanotechnology means the production of pharmaceutical products with dimensions ranging from 1 to $100 \mathrm{~nm}$. It is used to control drug release and increase its solubility. In this method, the plants used in the phytosome form or encapsulated forms such as a liposome. Many of the materials that come from the plants, such as flavonoids, play an important role in antiinflammatory and antioxidant activities and inhibit the activity of acetylcholinesterase enzyme which increases communication between nerve cell. If medicinal plants are used in the form of nanomedicine, they will absorb better and increase the stability of drugs and biocompatibility and reduce the dose required and side effects. These drugs improve cognitive functions such as episodic memory, language, and executive abilities. The limitation of herbal nano drugs is that quickly passes through the membrane and its toxicity is not fully understood. If their size is inappropriate, it creates problems in the capillary and renal system. Conclusion: In conclusion, because the failure of pharmacotherapy in the treatment of $\mathrm{AD}$, nanotechnology should be combined with plants to reduce the adverse effects and also drug delivery to the brain will be more effective.

\section{P195}

Speech and Language Impairments as the Primary Presentation of Creutzfeldt Jakob Disease

\section{Azadeh Abedinzadeh ${ }^{1}$, Behnoosh Tahanzadeh ${ }^{2}$}

${ }^{1}$ Faculty of Rehabilitation, Ahvaz Jundishapur University of Medical Sciences, Ahvaz, Iran

${ }^{2}$ Department of Speech Therapy, Faculty of Rehabilitation, Ahvaz Jundishapur University of Medical Sciences, Ahvaz, Iran

The Neuroscience Journal of Shefaye Khatam, 2019; 7(3): P195

Introduction: Creutzfeldt-Jakob disease (CJD) is a neurodegenerative disorder and caused by a proteinaceous infectious agent called a "prion" results in the accumulation of prion protein (PrPsc) in the brain. Reports of this disease include people of every two gender and are more frequent in the fifth and sixth decades of life. Given that the population goes to aging, the study of the diseases of this period finds special significance and given the progressive nature of the CJD disease, attention to symptoms is very important for early diagnosis and early treatment. Rapidly progressing dementia, ataxia, muscle tone alterations, and myoclonus are the manifestations of this disease, however, often CJD is associated with atypical clinical presentation and/ or inconclusive initial workup. Some studies reported speech and language signs in CJD patients and has been found one of the initial manifestation of CJD was language and speech difficulties that progressed worse. Linguistic symptoms in these patients include some types of aphasia, especially progressive aphasia over time, and dysarthria, apraxia, and stuttering are mentioned for speech symptoms. Conclusion: Speech and language problems in patients with CJD are important from this 
point of view, which can be detected at the first step of the disorder, therefore, consideration of speech and language problems that progressively worsen along with other symptoms associated with CJD can be a guide to early diagnosis of this disease.

\section{P196}

Efficacy of Balance Training in Falling and the Improvement of Postural Control in Parkinson Disease: a Review of Literature

\section{Narges Shakerian ${ }^{1}$, Anahita Hashempoor ${ }^{2}$}

${ }^{1}$ Student Research Committee, Faculty of Rehabilitation, Ahvaz Jundishapur University of Medical Sciences, Ahvaz, Iran

${ }^{2}$ Student Research Committee, Faculty of Medicine, Ahvaz Jundishapur University of Medical Sciences, Ahvaz, Iran

The Neuroscience Journal of Shefaye Khatam, 2019; 7(3): P196

Introduction: Parkinson disease(PD) is a progressive neurodegenerative disorder which manifests through symptoms including bradykinesia, gait difficulties, and rigidity. Postural instability which is the main reason for falling is also common in PD. The balance training program is undertaken as a plan of treatment to reduce these deliberating symptoms, so the porpuse of this study is accumulating the evidence on the role of balance training in falling and amelioration of postural control in patients with PD. We extracted the articles from PubMed and Scopus by use of standard search MESH terms including Parkinson disease, balance training, falling and postural control. Not only the postural response has been improved, but treatments focused on balance also attenuated the rate of falling in patients with PD. The application of challenging balance training exercises exerted a reduction in fear of falling during daily living activities. Conclusion: Furthermore, two of the articles assessed home-based balance training claimed to improve the postural control via increasing the time of single leg stance and stride velocity of gait. Overall different types of balance training with or without other treatments, are beneficial approaches to decline the manifestation of PD. Considering all aspects of different balance training in patients with cognitive problems and severe grades of PD would be the purpose of future studies as it has not been investigated seriously.

\section{P197}

Role of Glial Fibrillary Acidic Proteins (GFAP) in Alexander's Disease

\section{Moosa Javdani ${ }^{1}$, Abolfazl Barzegar Bafrouei ${ }^{2}$}

'Department of Clinical Sciences, Faculty of Veterinary Medicine, Shahrekord University, Shahrekord, Iran

${ }^{2}$ Faculty of Veterinary Medicine, Shahrekord University, Shahrekord, Iran

The Neuroscience Journal of Shefaye Khatam, 2019; 7(3): P197
Introduction: In addition to neurons, there are various protective cells such as astrocytes which allocate an important part of both central and peripheral neural tissue. These cells are to times more than neurons, and have different types. Astrocytes are responsible for a wide range of tasks in nervous system. It sets neurotransmitter system, synaptic information, energy metabolism, disposal of antioxidant and inflammatory responses. There are two types of astrocyte; fibrous astrocytes and protoplasm astrocyte named velvet astrocytes. Glial fibrillary acidic proteins (GFAP) is an acid protein that in normal situation exists in both of astrocyte. Glial fibrillary acidic protein is an intermediate filament protein that is expressed by numerous cell types of the central nervous system (CNS) including astrocytes and ependymal cells during development. Although it's more in fibrous astrocytes and it increases in neural damages. Children with Alexander disease develop, psychomotor retardation, seizures and a leukoencephalopathy with macrocephaly, leading to death usually within the first decade; patients with juvenile or adult forms typically experience bulbar signs, ataxia and spasticity, and a more slowly progressive course. The pathological Sign of all types of Alexander disease is the existence of Rosenthal fibers, cytoplasmic inclusions in astrocytes that contain the intermediate filament protein GFAP in association with small heat-shock proteins. Mutation in the encoding gene of the GFAP leads to a fatal disease called "alexander disease". Researches have shown that it's possible to control GFAP. This control will improves the patients suffering alexander disease. Alexander disease has depends directly on GFAP and a rare genetic disorder. Conclusion : GFAP levels are already used as a marker of neurologic injure in adults who suffer strokes and traumatic brain damage .

\section{P198}

A Brief Description a Bout Nanotherpy for Alzheimer's Disease

\section{Maryam Etemadi}

Department of Basic Sciences, Faculty of Biology, University of Zabol, Zabol, Iran

The Neuroscience Journal of Shefaye Khatam, 2019; 7(3): P198

Introduction: 46/7 million people suffer from Alzheimer's which is estimated to reach $131 / 5$ million by 2050. Alzheimer's is a neurodegenerative disorder(ND) that is identified by the presence of amyloid plaques in brain as the main Symptoms. Blood Brain Barrier (BBB), is one of the most important challenges to drug delivery for such as Alzheimer's, which limits the therapeutic effects. Hence, the development of Therapeutic systems based on nanoparticles(NPS), allows better and more targeted release for brain as a new strategies for brain disorders. The available drugs for Alzheimer's treatment only reduce the symptoms and prevent the progression of 
the disease, but definitive treatment for Alzheimer's has not identified yet, and BBB is one of the barriers to drug delivery to the brain. Nanomedicines can be introduced as an effective way to treat Alzheimer's. the ideal feature of NPS for the treatment of Alzheimer is crossing beyond $\mathrm{BBB}$ and establishment of an appropriate interaction with markers. Nanotechnology uses special polymer nanomaterials to design nanomaterials with the smallest application size that are capable to interact with biological systems at the molecular level, Which is responsible for stimulating, responding, interacting with target cells or tissues by inducing the desired physiological responses with the least side effects. Different classes of nanostructures can help improve the treatment through a gene or cell. Conclusion: As the nervous disorders are caused by several factors in NDs, the death of a specific type of neuron is caused by a cascade of several molecular and cellular events, nanotherapy and side effects of drugs in each of these pathways should be investigated. In vivo investigation can reveal long term effects of NPS, toxicity and their side effects. Early diagnosis of Alzheimer's results in more effective treatment.

\section{P199}

Infectious Agents and Neurodegeneration, HSV-1 and HHV-6 in Alzheimer's Disease

\section{Seyyede Nafise Vakili', Mohamadreza Diba ${ }^{2}$, Zahra Meshkat ${ }^{3}$}

${ }^{1}$ Department of Virology, Faculty of Medicine, Shiraz University, Shiraz, Iran

${ }^{2}$ Department of Biochemistry, Faculty of Medicine, Shiraz University, Shiraz, Iran

${ }^{3}$ Department of Microbiology and Virology, Faculty of Medicine, Mashhad University, Mashhad, Iran

The Neuroscience Journal of Shefaye Khatam, 2019; 7(3): P199

Introduction: Many studies were demonstrated that chronic bacterial and viral infections as possible risk factors for neurodegenerative diseases, including Parkinson's disease, amyotrophic lateral sclerosis and Alzheimer's disease. Infections of the central nervous system may produce multiple damages in infected and neighbouring normal cells. The activation of immune responses and inflammatory processes cause chronic damage resulting in alterations of neuronal function and viability. Viral agents have been reported to produce and deposit of molecular hallmarks of neurodegeneration, such as misfolded protein aggregates, oxidative stress, deficient autophagic processes, synaptopathies and neuronal death. Also, these effects may act in synergy with other risk factors, such as aging, concomitant metabolic diseases and the host's specific genetic signature in patients. Much evidence demonstrates that pathogens directly and indirectly induced Alzheimer's disease pathology, including amyloid accumulation, phosphorylation of tau protein, neuronal injury, and apoptosis. Many studied were showed that herpes simplex virus type ' (HSV'-) is a major risk for Alzheimer's disease (AD). This concept proposes that latent HSV' - in the brain of carriers of the type $₹$ alleles of the apolipoprotein $\mathrm{E}$ gene is reactivated by different events such as immunosuppression, peripheral infection, and inflammation, the consequent damage accumulating, and culminating eventually in the development of AD. Also, Human herpesvirus type 1 (HHV ${ }^{-}$) is another virus investigated as a potential contributor to Alzheimer's disease. However, it is uncertain whether its presence and activity is a cause or a consequence of the disease and it may be that $\mathrm{HHV}^{\top}$ - merely exacerbates the potentially harmful effects of HSV'- in patients. Conclusion: This review will focus on the role of HSV1 and HHV-6 in Alzheimer>s disease progression.

\section{P200}

\section{Manganese and Neuroinflammation}

\section{Seyedeh Negar Assadi}

Social Determinants of Health Research Center, Mashhad University of Medical Sciences, Mashhad, Iran

The Neuroscience Journal of Shefaye Khatam, 2019; 7(3): P200

Introduction: There are a few toxins can cause neuroinflammation in the environment. One of these toxins is manganese as a toxic metal in some doses. This study try to find the neurological effects of manganese in the neuroinflammation field. In some references researchers studied about the neurological effects of toxic metals such as lead and mercury but a few worked about the manganese. Toxicological and environmental medicine references had been reviewed such as agency for toxic substances and disease registry and environmental protection agency. These are showed neurological effects of manganese in moderate to high doses of exposure. Specially inhalational exposure in the environment and workplace. Manganese can cause neuroinflammation, synaptic injury, neurotransmitter disturbances, parkinsonism and parkinson's disease. The patient has tremors, disruption of movement, problems in walking and rigidity. Prevention is included use of perfect ventilation systems and suitable respirators with specific cartridge in inhalational exposure. Prevention of eating or swallowing things with moderate to high dose of manganese and it's compound. Prevention of skin contact by attention to hygiene guidance and wearing of specific gloves. If the patient is diagnosed in early stage of toxicity he or she get out from exposure and will be healthy. Screening of toxicity by biologic monitoring must be recommended. Blood sample in specific and periodic times can be helpful for this reason. Conclusion: Manganese can cause neuroinflammation and damage synaptic activity and neuronal disruptions. Prevention of exposure to moderate to high level of 
manganese must be implemented.

\section{P201}

Astrocytes: Consequences for Neuroinflammatory Pathogenesis of Alzheimer's Disease

Negar Khodaeinia ${ }^{1}$, Zahra Farjami ${ }^{1,2}$, Amirhossein Ashnaei $^{2}$, Neshat Ebrahimi ${ }^{3}$, Zeinab Sadat Hosseini ${ }^{4}$

${ }^{1}$ National Research Center of Genetic Engineering and Biotechnology (NIGEB), Department of Medical Genetics, Tehran, Iran

${ }^{2}$ Department of Modern Sciences and Technologies, Faculty of Medicine, Mashhad University of Medical Sciences, Mashhad, Iran

${ }^{3}$ Laboratory of Cedars-Sinai Medical Center, Los Angles, California, USA

${ }^{4}$ Student Research Committee, Mashhad Branch, Islamic Azad University, Mashhad, Iran

The Neuroscience Journal of Shefaye Khatam, 2019; 7(3): P201

Introduction: Alzheimer's disease (AD) is a neurodegenerative disease with main clinical signs of dementia and cognitive impairment. Neuroinflammation is involved in the onset of numerous neurodegenerative disorders. Astrocyte is the most common kind of glial cells in the central nervous system (CNS) and seems to be involved in the induction of neuroinflammation. Traumatic conditions make astrocytes to overexpressed proinflammatory chemokines and cytokines, which are related to the pathogenesis of $\mathrm{AD}$. Cytokines and associated molecules have significant roles in both neuroprotection and neurodegeneration in the CNS. During early $\mathrm{AD}$ pathogenesis, amyloid beta $(\mathrm{A} \beta)$, S $\cdots$ B and IL $-\beta$ could take about a vicious cycle of $A \beta$ generation between astrocytes and neurons that is leading to progressive and chronic neuroinflammation. In progressive stages of AD, TRAIL secreted from astrocytes have been revealed to bind to death receptor - (DR $\left.{ }^{\circ}\right)$ on neurons to activate apoptosis in a caspase- $\Lambda_{\text {- }}$ dependent way. Also, astrocytes could be reactivated by TGF $\beta$ \ to make more $A \beta$ and to undergo the aggravating astrogliosis. TGF $\beta^{r}$ was also detected to cooperate with $A \beta$ to make neuronal demise. Conclusion: According to the above expressions, it is probable that treatments effective at neuroprotection also improve astrocyte-neuron interactions. Though, this possibility has not been completely studied. Astrocytes secreting chemokines and cytokines in the early stage of AD make a novel potential therapeutic target and suggestion the possibility of developing novel medications to cure AD by intervening neuroinflammation.

\section{P202}

The Effect of Herbal Medicines on the Treatment of Multiple Sclerosis

Mahdi Ardakani ${ }^{1}$ Mohammad Salari ${ }^{2}, \quad$ Ali
Honarmand $^{2}$ Rasul Saberi ${ }^{2}$

${ }^{1}$ Department of Medicine, Faculty of Medical Sciences, Islamic Azad University, Mashhad, Iran

${ }^{2}$ Department of Biology, Faculty of Basic Sciences, Islamic Azad University, Mashhad, Iran

The Neuroscience Journal of Shefaye Khatam, 2019; 7(3): P202

Introduction: Today, due to the widespread and complex symptoms of multiple sclerosis and side effects after chemotherapy And stem cell transplantation, the use of medicinal herbs and supplements can be very effective. According to a study conducted at Pubmed and Google Scholar, from 2014 to 2019,78 articles were found with entry criteria, of which 18 articles showed positive results and were examined.Recent studies have shown that compounds in some plants such as sulforaphane (SFN),flavonoids, icariin and berberine have antineopatologic,anti-oxidative and anti-inflammatory properties And the ability of some of them to prevent side effects due to endometrial endometrium reticulum (ER), hydrogen peroxide, benomotanic acid and homocysteine Can be used as preventive and therapeutic agents for central nervous system (CNS) disorders.Medicinal plants such as Epimedium, Achillea millefolium, Androg raphiculata, Ruta graveolens, Aloysia citrodora, Ginkgo biloba,MS14,Gastrodia elata, Vitis vinifera, Piper methysticum, Vaccinium macrocarpon,Nigella sativa,Zingiber officinale, Curcuma longa and Hypericum perforatum have different therapeutic effects in treating MS. Also, Chinese Medicinal Plants (CHM) such as Radix glycyrrhizae, Ordinally radix Angelicae sinensis, Bombyx Batrycatus and Tripterygium wilfordii Hook $\mathrm{f}$ (TwHF) have shown better results than the western methods of treatment for annual returns and annual period and reduces the side effects of taking chemical medications such as interferon beta. Vitamin D, Melatonin, Carnitine and Coenzyme Q10 can also be used as supplements for boosting the immune system and vitamin B12 in the CNS function. Conclusion: It can be said that the use of herbal medicines and foods that contribute to the functioning of the central nervous system in the early stages of multiple sclerosis can slow down or even stop the disease. Also, using these drugs and supplements can avoid the side effects of chemical drugs Of course, in this treatment, it is important to recognize early illness.

\section{P203}

New Horizons to Manage Epilepsy Based on an Integrated Comprehension of Complex Systems Theory and Persian Medicine

\section{Majid Anushiravani, Nima Mafakheri', Zeinab Jaberzadeh ${ }^{3}$}

${ }^{1}$ Department of Persian Medicine, Faculty of Persian and Complementary Medicine, Mashhad University of Medical 
Sciences. Mashhad, Iran

${ }^{2}$ Center for Computational Neuroscience Research, Department of Biomedical Engineering, Imam Reza International University, Mashhad, Iran

${ }^{3}$ Department of Psychology, Torbat-e Jam Branch, Islamic Azad University, Torbat-e Jam, Iran

The Neuroscience Journal of Shefaye Khatam, 2019; 7(3): P203

Introduction: Medical management of Epilepsy can be based on two different theoretical explanatory models of the brain behavior. Conventional approach to the brain is based on linear causality, certainty, reductionistic analytic modeling, and one-to-one correspondence between brain structures. Another approach is based on complex systems theory that explains the brain behaviors relying on circular causality, uncertainty, holistic emergent modeling, and one-to many/many-to-one correspondence between the brain and other organs. The first approach is the basis of current clinical interventions to control epilepsy. The latter approach understands the neurologic phenomena as highly-complex events arising from dynamic interactions and counteractions in the brain and its extensive inner and outer exposome. In this viewpoint the brain is a master organ involved in a web of interacting organs with various types of biologic information including mechanical, neurochemical, hormonal, electromagnetic, etc. so all the elements of the human bio-system including the organs, energy, and information are needed to provide multi-stability in both physiological and pathological states of the brain. Additionally sage physicians in Hikmat-based Persian Medicine like Avicenna have presented wide interactions between the brain and other organs including gut, uterus, heart, liver, spleen, etc., and the great influence of their healthy/unhealthy dynamics on the brain behavioral patterns. Converging the conceptual principles of complex systems theory, ethio-semiologic teachings about epilepsy in medical manuscripts of Persian Medicine, and also regarding the recent supporting evidences on organ-brain axes, our understanding about epilepsy process, seizure susceptibility and epileptogenesis would shift to a different perspective. Conclusion: Our medical experiments in academic clinical settings show the considerable outcomes of this interdisciplinary insight to promote medical treatments and improve the quality of life in epileptic people.

\section{P204}

Introns That Make Change; MicroRNAs and Synaptic Plasticity

Behzad Ensan, 2, Mohammad Bagher Soltanian, 2, Mahshid Safari Ghalezou, 2, Nima Zafari', 2, Parsa Bathhaee $^{1,2}$

${ }^{1}$ Student Research Committee, Faculty of Medicine, Mashhad University of Medical Sciences, Mashhad, Iran

2Department of Neurology, Faculty of Medicine, Mashhad
University of Medical Sciences, Mashhad, Iran

The Neuroscience Journal of Shefaye Khatam, 2019; 7(3): P204

Introduction: Synapses are functional units, transfer and store information within nervous system. Synaptic plasticity is characterized as modification of synapses in response to stimulation they receive. Functional plasticity refers to electrophysiological or chemical signaling, while structural plasticity denotes fundamental changes of number or structure of neurons. This process constitutes underlying mechanism in memory, learning and also drug addiction. Furthermore, synaptic plasticity plays an essential role in post-stroke rehabilitation. As well as many other mechanisms, synaptic plasticity originate from neuronal-plasticity genes. Small, noncoding miRNAs emerged as principal regulators, exert their influence beyond transcription. Although rarely they can promote translation, miRNAs predominantly function as post-transcriptional inhibitory molecules. These molecules base-pair with corresponding sequences in 3'-UTR within mRNA and either suppress mRNA translation or promote deadenylation and degradation of them. Plenty of these bio-molecules have been detected in mammalian brains, participating in various functions. miRNA and precursor-miRNA has been identified in axons and dendrites, far away from the soma. They control the expression of NMDA receptors, AMPA receptors and signaling molecules. In addition, miRNAs can be locally regulated by synaptic activity. For instance, recent evidence suggests that NMDA receptors modulate processing of dendritic precursormiRNA. Besides functional plasticity, contribution of miRNAs like miRNA-188 and miRNA-132 to dendritic spine number and morphology has been demonstrated. These findings are in accord with considerable literature signifies the role of miRNAs in long-term potentiation (LTP) and long-term depression (LTD). Recently, miRNAs have drawn great deal of attraction. Despite considerable number of identified miRNAs, just a few of them have been investigated functionally. As a gene is targeted by multiple miRNAs and synaptic activity alters several miRNAs simultaneously, new laborious technologies are required to be developed. Conclusion: Since these bio-regulators possess undeniable role in neuroplasticity, they can be reliable pharmaceutical targets for treatment of addiction, stroke and other neurodegenerative diseases.

\section{P205}

A Brief Description about Alzheimer's Disease and Gene Therapy

\section{Maryam Etemadi}

Department of Basic Sciences, Faculty of Biology, University of Zabol, Zabol, Iran

The Neuroscience Journal of Shefaye Khatam, 2019; 7(3): P205 
Introduction:Alzheimer's disease is a neurodegenerative disorder. Neuropathologic symptoms of Alzheimer's are extracellular amyloid plaques and intracellular phosphorylation of tau proteins. Gene therapy is a new form of molecular medicine will have a great impact on human's health in the next century. A gene therapy technique mediated by a vector-driven genes, transfer DNA or RNA to target cells and increase the expression of therapeutic proteins or protectors, sometimes silence certain virulence genes. Gene delivery and stable production is the Achilles Heel in gene therapy methods. Since amyloid- $\beta$ is continuously produced in the brain, the amount of production and degradation of it should be reached stable. Gene therapy can directly increase enzymatic activity, through the transfer of a recombinant gene by the vector. Most vectors are viruses and the gene is expressed within it. One types of gene therapy is gene transferring to engineered cells and then inject them into the central nervous system. The unclear Alzheimer's main pathogen is one of the limitations of gene therapy. An effective gene therapy needs seeking for main target genes. With the completion of the human genome project and the discovery of important genes, we are nearly close to the definitive Alzheimer's treatment. Exception of safety, other important aspects of gene therapy for alzheimer's include the correct gene dosage and the proper targeting of gene expression. Conclusion: More recognition of viruses, let us improve and modify highly site-specific expression and adjustable expression level.

\section{P206}

The Effect of Psychological Interventions on Improving the Working Memory of Children with Epilepsy

\section{Sahar Sadat Nazm Bojnourdi}

Department of Cognitive Sciences, Cognitive Psychology, Faculty of Psychology and Educational Sciences, Mashhad University of Medical Sciences, Mashhad, Iran

The Neuroscience Journal of Shefaye Khatam, 2019; 7(3): P206

Introduction: Working memory is a temporary system that retrieves information during a cognitive assignment such as perception, learning, argumentation of long-term memory. In the study of people with epilepsy, the focus of which is the hippocampus, memory retrieval has undergone significant changes. This can be attributed to the destruction of hippocampal synapses during epileptic seizures. The purpose of this paper is to investigate the effect of working memory rehabilitation on the improvement of synapses in children with epilepsy based on research evidence. This case-control study was performed on 30 children with epilepsy with an average of 10 years. Subjects were examined using available sampling and diagnosis of neuropathologist. Working memory performance through error score using work memory (n-back test) and executive functions that included error components, phase response time Third, the Stroop test and the number of Wisconsin test card categories were determined. After two months and an average of 8 hours of work with a training test, which is a n-back exam test, cognitive rehabilitation and memory enhancement strategies as games and entertainment, subjects were re-tested with n-back tests. The results showed that there was a significant difference between the working memory of patients. Neurogenesis can be an effective factor in positive changes in working memory, which is closely related to rehabilitation in the process of treating the brain as a whole unit on its own position, the hippocampus. Brain application areas are likely to allow neurons to be retrieved and expanded during the rehabilitation process. Conclusion: Given the available evidence, rehabilitation in children with epilepsy can be considered as a route to improve memory processes, which structurally increases the chance of plasticity and the production of new synapses.

\section{P207}

\section{Investigating the Relationship Between Migraine and Menopause}

Mahya Azimi', Farahnaz Farnia ${ }^{2}$, Arefeh Khaleghi', Seyed Matin Arman ${ }^{1}$, Mohsen Lazemi', Mohadese Taghi Molaei ${ }^{1}$

${ }^{1}$ Student Research Committee, Faculty of Nursing and Midwifery, Shahid Sadoughi University of Medical Sciences, Yazd, Iran ${ }^{2}$ Department of Nursing, Faculty of Nursing and Midwifery, Shahid Sadoughi University of Medical Sciences, Yazd, Iran

The Neuroscience Journal of Shefaye Khatam, 2019; 7(3): P207

Introduction: Migraine is a cerebrovascular disorder characterized by frequent and progressive headaches and is reported to be three times more likely than men in women.Menopause means permanent cessation of menstruation and is associated with hormonal changes. Menopause may have an effect on this complication. Therefore, the purpose of this study is to review the research carried out in this field. This review study was conducted by searching the Science Direct, Scopus, PubMed, and Google scholar databases using the "migraine" menopausal keyword "Women" during the 2009 to 2019 returns. Non-English articles or main purpose They were headaches and were excluded As a result, 10 articles found entry conditions. There is a significant and positive correlation between migraine and menopause in most studies. In general, because of estrogen hormone stability during menopause, there is a potential for improvement in migraines. Of course, the type of menopause (natural-induced) also affects it, and people with spontaneous menopause experience a more favorable period of migraine, and in the postmenopausal phase (perimenopause) increases 
the probability of migraine attacks due to hormonal fluctuations. In a study, the effect of menopause on migraine is attributed to the vulnerability of individuals in this period, and another study states that many women believe that their headache, especially migraine, ends after menopause, which in many cases is not the case. Conclusion: According to the findings, it is concluded that during the menopause, the number of attacks decreased, but this number increased during the menopausal period, but further studies are needed due to the lack of studies.

\section{P208}

Investigating the Relationship between the Expression of e-5NT and the Development and Progression of Neurological Diseases: Systematic Review

\section{Zeinab Boostani, Mohammad Reza Khojasteh}

Student Research Committee, Mashhad Branch, Islamic Azad Univercity, Mashhad, Iran

The Neuroscience Journal of Shefaye Khatam, 2019; 7(3): P208

Introduction: Neurological diseases affect central nervous system with inflammation including parkinson and multiple sclerosis. Ecto-5'-nucleotidase (e-5NT) is an enzym that in humans is encoded by the e-5NT gene. E-5NT commonly serves to convert adenosine monophosphate to adenosine. Although up-regulation of e-5NT was repeatedly reported in several vivo models of brain injury, the regulation of its expression and function remains largely unknown. Therefore,the purpose of this study was to determine the relationship between the expression of e-5NT and the development of neurological diseases. We searched Pubmed, Google scholar, Cochrane, SID and ISC databases until December 2018 with neurological diseases, ecto-5'nucleotidase and development keywords. By searching these databases, only 25 articles were found in Pubmed and 100 articles were found in Google scholar. From 125 articles with investigating title we exclude 96 articles and with reading abstract we exclude 18 articles. Finally, we include 11 articles in to our study. 9 articles showed increasing the expression of e-5NT, leading to the development of neurological diseases, as well as changes in the level of the enzyme on neuroinflammation and immune function. The low level of activity for this enzyme in $\mathrm{T}$ cells can be related to continuous anomalies in the function of T cells in the clinical course of MS. In one article, inhibiting the formation of e-5NT may reduce musculoskeletal disorder in patients with epilepsy and provide new therapies for drug-resistant seizures in these patients. In another article, it was stated that a change in e-5NT expression could cause neuromuscular enhancement in myasthenia gravis with minimal disorder. Conclusion: Our findings imply that differential expression of e-5NT in astrocytes at different phases during the course of neuroinflammation may have a pivotal role in the induction, amplification and counterregulation of inflammatory response in the central nervous system. It is suggested that by blocking e-5NT receptors by selective antagonists, nerve stimulation can be eliminated in some neurological diseases.

\section{P209}

Interplays between Nociceptors and Immune Cells; Mast Cells

\section{Abolfazl Barzegar Bafrouei, Moosa Javdani ${ }^{2}$}

${ }^{1}$ Faculty of Veterinary Medicine, Shahrekord University, Shahrekord, Iran

${ }^{2}$ Department of Clinical Sciences, Faculty of Veterinary Medicine, Shahrekord University, Shahrekord, Iran

The Neuroscience Journal of Shefaye Khatam, 2019; 7(3): P209

Introduction: In response to damage, resident immune cells are activated and blood borne immune cells are recruited to the site of damage. Immune cells also initiate the sensitization of peripheral nociceptors. The immune system also reduces sensitization by producing immune-derived anti-inflammatory and analgesic or proresolution agents. Mast cells are granulated denizen immune cells that are separate into connective tissue and mucosal subtypes and are locate close to capillaries. Mast cells degranulation needs direct interaction between peripheral nerve terminals mast cells, which is indirect by the calcium-dependent cell adhesion molecule $\mathrm{N}$-cadherin. They participate in innate host defense and allergic excitations and are de-granulated within a few minutes of an inflammatory reaction, resulting in liberate of bradykinin, histamine and other mediators that association to vasodilation. Once activated by damage, vascular endothelial cells, and immune and immune-related cells such as keratinocytes also synthesize and exude anti-inflammatory cytokines, pro-resolution lipid mediators and opioid peptides to suppress pain. Mast cells are contribute to nociceptor sensitization in a number of contexts and locate close to primary nociceptive neurons. Reported injection of the secretagogue compound $48 / 80$ promotes degranulation of mast cells in the dura and leads to stimulation of meningeal nociceptors. Mast cell degranulation also contributes to the fast onset of nerve growth factorinfluenced thermal hyperalgesia. Pelvic pain associated with neurogenic cystitis was removed in mice missing mast cells. Conclusion: Although these findings demonstrate that resident mast cells sensitize peripheral nociceptors, it is unclear which chemical mediator(s) branched from mast cells are basic for this effect.

P210

Dementia and Persian Medicine

Sadegh Shokri 
Department of Persian Medicine, Faculty of Persian Medicine, Birjand University of Medical Sciences, Birjand, Iran

The Neuroscience Journal of Shefaye Khatam, 2019; 7(3): P210

Introduction: As the increased incidence and mortality of noncommunicable diseases and changing the age pyramid of societies towards old age, the prevalence and complications of dementia are increasing. Regardless of the negative and harmful effects of this disorder on the quality of life and the imposition of high costs, effective treatment has not been found so far. At the same time as the global utilization of other medical schools, such as traditional and complementary medicine, due to the chronic nature of the disorder, patients and their relatives also turn to these medical and seek ways to treat and reduce their complications. Traditional Persian medicine (TPM) with its long and ancient history also provides ideas and strategies about this disorder. The purpose of this article is to examine these ideas and approaches and adapt it to the up to date scientific evidences .The (TPM) is based on the theory of humors and temperament. From this perspective, dementia, and particularly Alzheimer's disease, may be considered as a disease with a cold physiopathological mechanism.If this adaptation is correct, management of this disorder will mainly be based on lifestyle modification and some traditional treatments and non-medical interventions .Modify nutrition and nutrition content, modify physical activity, sleep modifying, modify mental and emotional events, environment improvement, and take medications such as lemon balm, lavender, grape(currant), sweet almonds, Pistachios, Fig, cinnamon, olive, chufa sedge, Chamomile, hazelnut and Boswellia depending on the responsible mechanism have been raised Most of them have an acetylcholine esterase inhibitor effect and may be effective on dementia Conclusion: Revising the scientific texts of (TPM) and adapting it to new findings may offer new solutions to this disorder.

\section{P211}

Review of the Anti-Inflammatory Effect of Antioxidants on Multiple Sclerosis

\section{Hamideh Asadinezhad}

Jahrom University of Medical Sciences, Jahrom, Iran

The Neuroscience Journal of Shefaye Khatam, 2019; 7(3): P211

Introduction: Multiple sclerosis (Ms) is a chronic autoimmune disease in the central nervous system, which is a demyelinating disorder due to myelin involvement. It affects more than 2.3 million people in the world, often aged between 20-40, and is increasing among the young population. The underlying cause of MS is still unclear. However, genetic factors, environmental contamination, and the E-B virus are among the factors mentioned. Research conducted in recent years suggests that the main causes of nerve scarring, can be free radicals such as reactive oxygen species (ROS) and reactive nitrogen species (RNS). The source of (ROS) and (RNS) can be the intrinsic factors, such as mitochondrial activity, phagocytic cells or external factors such as contamination, tobacco smoke, heavy metals, some drugs Like Halothane. The human body with antioxidant enzymatic and non-enzymatic systems cleans up these agents. free radicals, especially ROS, in case of excessive growth in the body, cause nerve inflammation in the site, and in addition to increasing monocytes and Macrophages it causes functional impairment in the blood-brain barrier (BBB). On the other hand, these factors increase Tcell and Bcell and ultimately lead to demyelination. The research materials were extracted from SID, Science Direct, Google Scholar from 20 papers using Multiple Sclerosis, Neuronal Inflammation, Oxidative Stress, Demyelination, Antioxidant therapy. Conclusion: External antioxidants such as flavonoids, Carotenoid, selenium, etc. can help the body's antioxidant systems to more effectively remove the free radicals from the body, so antioxidants, by their anti-inflammatory properties, reduce the rate of demyelination and degradation in the area. These materials also modify immune system activity and prevent frequent attacks of the immune system to the nerves. According to the statement, the use of appropriate antioxidants can be useful in treating MS.

\section{P212}

Increased Risk of Ischemic Stroke among Patients with Multiple Sclerosis: A Systematic Review

\section{Melika Farshidianfar, Fahime Akbarpour}

Student Research Committee, Islamic Azad University of Medical Sciences, Mashhad, Iran

The Neuroscience Journal of Shefaye Khatam, 2019; 7(3): P212

Introduction: Multiple sclerosis is known to be an autoimmune disease that reduces the life expectancy of patients with comorbidities such as ischemic stroke. The aim of this systematic review is to estimate the increased risk of ischemic stroke in patients with multiple sclerosis. Literature review was conducted using bibliographic databases, Pubmed, Google Scholar, SID, Iranmedex and ISC (from April 2014 to March 2019) on studies related to increased risk of ischemic stroke in patients with multiple sclerosis. One reviewer abstracted data using a standardized data collection form, while the second reviewer verified the abstraction. From 620 articles that were reviewed by title, 29 were extracted and after reading the abstractions, five studies were included in the study. Five cohort studies with a total of approximately 35 thousand MS patients and a minimum of three years follow-up were included. Only one study was particularly focused on the risk of ischemic stroke and others studied cardiovascular diseases and comorbidities in MS patients. A 
New York City based study investigated vascular disease among hospitalized multiple sclerosis patients. They reported that MS patients were less likely to be hospitalized for ischemic heart disease but more likely to be hospitalized for ischemic stroke. Overall, MS patients had higher incidence of ischemic stroke and hospitalization compared to the control groups. Conclusions :This literature review concludes that MS patients are at a higher risk of ischemic stroke. Nonetheless, there is still insufficient understanding of the nature of these findings. Therefore, we suggest in-detailed studies designed to understand the high prevalence of ischemic stroke amongst MS patients.

\section{P213}

\section{Forest Fire in Human Brain}

\section{Fatemeh Babaie}

Cellular and Molecular Biology Student Biology Group, Basic Science Department, Faculty of Cellular and Molecular Biology, Semnan University, Semnan, Iran

The Neuroscience Journal of Shefaye Khatam, 2019; 7(3): P213

Introduction: Despite recent failures in the development of targeted drugs for Alzheimer's disease, If you try to just treat plaques in those people, it's like trying to put out forest fire by blowing out a match and it means that the drugs is not enough. Some people can have brains clogged with amyloid plaques and tau tangles and still think and behave perfectly normally. What made those people resilient was lack of neuroinflammation. Conclusion: This study explored the effects of including of gamma and theta wave on the human brain. This involved a group of 42 adults, aged 20 to 29, and 42 others aged 60 to 76 years. When the gamma and theta interact the over-60s suddenly had performances similer to those under 30 . The test includes exercises consisted of images presented every few second. Also an ABA single-subject design was used to evaluate the effects of $40 \mathrm{~Hz}$ flickering light inside prototypic goggles on the power spectral density of gamma waves in brain of the subject measured by 64-channel EEG (Electroencephalogram).

\section{P214}

Investigation of Microrna-326 Changes in Multiple Sclerosis Patients: Systematic Review

\section{Parsa Shoqi ${ }^{1,2}$, Mohamad Reza Khojasteh ${ }^{1,2}$, Valareza Alizadeh ${ }^{1,2}$}

${ }^{1}$ Student Research Committee, Mashhad Branch, Islamic Azad university, Mashhad, Iran

${ }^{2}$ Department of Mashhad Neuroscience Research, Mashhad Branch, Islamic Azad University, Mashhad, Iran
Introduction: Multiple Sclerosis (MS) is an inflammatory autoimmune disease with immune related pathogenesis process that caused myelin degeneration of the central nervous system(CNS). microRNAs(miRNAs) are class of short, non-coding RNAs that play role in post-transcriptional gene expression. Studies showed that miRNAs play important roles in autoimmune disease by participating in differentiation and maturation of immune cells. miR-326 identified as a fundamental key for developing of T cell and differentiation of Th17. We searched PubMed and science direct search engines with the miR-326 and Multiple Sclerosis key words. We found 17 articles in PubMed until April 2019 and after reading the titles and abstracts and excluding reviews, we include 8 articles. There were 26 articles in science direct since 2015 till April 2019 with research articles filter that after reading titles and abstracts there were only 3 articles left. Studies assess the level of miR-326 in serum of MS patients and all the articles except one of them found overexpression of miR-326 in MS patients compared to controlled group. In one studies announced that relapse and remit phase of MS are distinguishable on the basis of miR-326 in serum. In some treatment methods (natalizumb and glucocorticoid) lower level of miR-326 showed after treatment. Most of the studies suggested that due to difficulties of current diagnostic methods of MS that based on symptoms, MRI and CSF analysis, we can evaluate miR-326 in patients' blood more convenient. Conclusion: on the basis of included articles, there is a correlation between MS and overexpression of miR-326. So miR-326 is a good biomarker for prognosis and monitoring of MS patients. Because of convenient detection of miRNAs as biomarkers for MS we suggest more studies in this field for other miRNAs which may have the same role.

\section{P215}

Combination of Exosomes, Gene Vectors and Stem Cells, As A Novel Therapy for Neuroinflammation

\section{Masood Ghodsi Moghadam}

Faculty of Medicine, Mashhad University of Medical Sciences, Mashhad, Iran

The Neuroscience Journal of Shefaye Khatam, 2019; 7(3): P215

Introduction: In the treatment field of neuroinflammation diseases, three themes attracted researchers: extracellular vesicles (particularly exosomes), gene delivery vectors and stem cells (SCs). These approaches were applied in many studies, alternatively. Although each was observed to have meaningful results, some problems like immunogenicity, passing the blood-brain barrier (BBB), tumor potential, remained. It seems that combining these major strategies makes treatment more effective. this article reviewed hybrid strategies utilized for treating Alzheimer, Parkinson, multiple sclerosis, as follows: 
Liposomes have some restrictions for plasmid, RNAs, proteins delivery. Liposomes cannot cross the BBB, may stimulate the immune system against themselves, thus having low-efficiency. Exosomes don't have these weaknesses and probably are better choice for treatment. Micro-vesicle associated AAV (adeno-associated virus) vectors were observed as a novel method for gene delivery since they are covered by membrane, they can be targeted for specific cells and escape from the immune system. SCs are increasingly used for cell formation in neurodegeneration. Moreover, by inducing genetic vectors into autologous SCs, they are modified (ex vivo) to be cellular carriers of the genes and be able to decelerate the disease process, too. In another study, scientists transfected M (mesenchymal)SCs with lentiviruses, therefore inside glioma cells medium, MSCs expressed shRNA for silencing Huntington protein. They observed that MSC-derived exosomes, acted as indirect shRNAs carriers. This approach used all of the three mentioned themes. Applying neural SCs with different vectors may result significantly in clinical trials. This article also hypothesized that exosomes may solve some challenges related to human induced pluripotent SCs usage. Special exosomes transfer tumor regression factors, so possibility of tumor development can be eliminated by them. Conclusion: Furthermore, SCs variability is another major challenge in tissue transplantation. Exosomes which carry epigenetic factors, induce better differentiation by reducing chance of variability.

\section{P216}

Correlation between Seizure and Arrhythmia in Children: A Review

\section{Zahra Rezaei ${ }^{1}$, Hosseinn Amini-Khoei ${ }^{2}$}

${ }^{1}$ Student Research Committee, Shahrekord University of Medical Sciences, Shahrekord, Iran

${ }^{2}$ Medical Plants Research Center, Shahrekord University of Medical Sciences, Shahrekord, Iran

The Neuroscience Journal of Shefaye Khatam, 2019; 7(3): P216

Introduction: Seizure is a sudden, excessive and abnormal electric discharge of brain cells that leads to disturbance in cortical function. It is evident that seizure can affect heart signals. A hypothesis expresses that seizure and arrhythmia both are manifestations of a channelopathy because heart and brain both have ion channels, but seizure relation with heart electrical activity in children is not understood well yet. ECG and Echocardiography in children with seizure show a significant decrease in LV systolic function, RV diastolic function and significant long TP intervals. Increased heart rate variability, beat-to-beat $Q_{-} T$ interval, and ventricular repolarization variability have also been reported. Anti N_methyl_d_aspartate receptor encephalitis (NMDARE) which includes seizure causes worsening of tachyarrhythmia, bradyarrhythmia, and asystole episodes. Left ventricular end diastolic dimension (LVEDD) and left ventricular systolic end dimension (LVESD) are Echocardiography findings in children with seizure. Hypocalcemia, an important etiology in seizure prolongs corrected Q-T. Dysautonomia is another common finding. In the other hand hypoglycemia induced by beta blockers in young patients with long Q T syndrome causes seizure and in these children death by hypoglycemic seizure occurs by using Implantable Cardioverter Defibrillator (ICD). Our results show a correlation between arrhythmia and seizure. Conclusion: The possibility of the occurrence of arrhythmia in children with seizure suggests heart condition and ECG monitoring as a rapid method for serum $\mathrm{Ca} 2+$ evaluation and prevention of seizureinduced sudden death.

\section{P217}

\section{The Role of Nutrition in Multiple Sclerosis: A Systematic Review}

\section{Mahdi Isazadeh, Mohammad Javad Sadeghi}

Student Research Committee, Faculty of Medicine, Aja University of Medical Sciences Tehran, Iran

The Neuroscience Journal of Shefaye Khatam, 2019; 7(3): P217

Introduction: Multiple sclerosis (MS) is a chronic, inflammatory, and autoimmune disease of the central nervous system (CNS), leading to widespread focal degradation of the myelin sheath, variable axonal and neuronal injury, and disabilities in young adults, mostly women. Recent research in preclinical models, epidemiologic studies, and limited prospectively followed cohorts provide preliminary evidence that dietary factors influence MS incidence, disease course and symptomatology. Mechanistic experiments highlight potential effects of diet on both immune modulatory and neurodegenerative processes in MS. There is not one MS treatment that works well for everyone, since people may have different symptoms. People with multiple sclerosis are at greater risk for being low in some vitamins and minerals, including the following: calcium and vitamin D, Vitamin B12, Selenium and Zinc. Many types of special diets have been suggested to help manage MS. Some special diets may be harmful because they include potentially toxic amounts of certain vitamins, or exclude important nutrients. That's why it's important to consult with patient's healthcare professional before starting any diet that includes nutritional supplements or vitamins. It seems that we can decide to help people stay healthy by providing nutritional guidance and physical activity opportunities. For the moment, there are only good prospects for improving the wellbeing of patients with MS. Since each person's experience with MS is a little 
bit different, we need to find a nutrition plan that works for people with multiple sclerosis. No special diets been proven to prevent MS or affect the way it may develop. Conclusion: A healthy lifestyle will benefit not only how patients feel physically but it can help emotional health too.

\section{P218}

Physiotherapy in a Patient after Multiple Meningioma Craniotomy: ACase Report

\section{Mahdieh Hamedfar, Mandana Rezaei, Hanieh Javan,} Zhinous Sheikholeslami

Department of Physiotherapy, Faculty of Rehabilitation Sciences, Tabriz University of Medical Sciences, Tabriz, Iran

The Neuroscience Journal of Shefaye Khatam, 2019; 7(3): P218

Introduction: Meningioma is one of the most common brain tumors that originate from arachnoid cells. The incidence rate is 6.59 per 100,000. Meningioma is more prevalent in females. Medical treatments include surgery, chemotherapy, radiotherapy, and physical therapy. The purpose of this study was to report physiotherapy in a patient after craniotomy of meningeal tumor. The patient was a 53-year-old woman with a history of craniotomy and discharge of meningioma in the left fronto-parietal lobe who referred to the physiotherapy clinic. The patient's chief complaint was an imbalance during walking and a major weakness in the lower extremity muscles. In the clinical evaluation, mild cognitive and perceptual impairments were identified. Other findings were postural, balance and walking impairments, need for assistance in most transfer activities, and muscle weakness mainly in the ankle dorsi flexors, hamstring, and quadriceps muscles. The range of ankle dorsiflexion movement was limited to $-35^{\circ}$. The patient had no sensory problems, but in the cranial nerves, the optic nerve was involved. Hypertonicity in ankle plantar flexors, shoulder internal rotators, abductors and flexors and elbow flexors was evident. The abnormal synergy patterns were not observed. The primary score of the patient's independence level was 60 based on the Barthel index. Twenty two sessions of physiotherapy were applied primarily to increase the independence level of the patient in daily activities. Reassessment at the end of the physiotherapy revealed significant improvement in the strength of lower extremity muscles which was followed by an increase in the range of ankle joint dorsiflexion $\left(-10^{\circ}\right)$. Hypertonicity was decreased in the involved muscles. Balance, posture, and transfer were also getting better scores. Conclusion: The score of the patient's independence level increased to 95. Rehabilitation, including physical therapy interventions, after brain tumor can be effective in improving function, quality of life and the patient's independence level.

\section{P219}

The Safety of Cannabinoid for the Treatment of Multiple Sclerosis

Amirhossein Ashnaei, Zahra Farjami', Neshat Ebrahimi $^{2}$, Negar Khodaeinia ${ }^{3}$ Zeinab Sadat Hosseini ${ }^{4}$

${ }^{1}$ Department of Modern Sciences and Technologies, Faculty of Medicine, Mashhad University of Medical Sciences, Mashhad, Iran

${ }^{2}$ Laboratory of Cedars-Sinai Medical Center, Los Angles, California, USA

${ }^{3}$ National Research Center of Genetic Engineering and Biotechnology (NIGEB), Department of Medical Genetics, Tehran, Iran

${ }^{4}$ Student Research Committee, Mashhad Branch, Islamic Azad University, Mashhad, Iran

The Neuroscience Journal of Shefaye Khatam, 2019; 7(3): P219

Introduction: The document for the therapeutic effect of cannabinoid in the treatment of Multiple Sclerosis(MS) is enhancing but is not as yet persuasive. Also, most of the tests have reported no considerable effect, the large part of the evidence that supports a useful effect on spasticity and pain is based on the subjective measurement in tests was likely to be a problem. The accessible clinical trial study offer that the adverse side effects related to using cannabinoid based medical extracts are generally mild. However, several of these tests were review a period of months and it is possible that other adverse side effects, not shown in these short term data, could increase with long term use. Further there is no document to offer that their effects on balance and motor control, or immune function, maybe clinically considerable. Also, there is reason to be concerned about the use of therapeutic cannabinoid people amenable to psychosis and by pregnant woman, given the enhancing document of their adverse effects on the embryo. Conclusion: The modest therapeutic effects of cannabinoid showed the risk of long term adverse side effects. There is reason to be continuous about their use in the treatment of MS.

P220

MS14 a Marine-Herbal Medicine, an Immunosuppressive Drug in Multiple Sclerosis: A Narrative Review

Mohammad Bagher Soltanian1, 2, Mahshid Safari Ghalezou $^{1,2}$, Nima Zafari ${ }^{1,2}$, Parsa Bathhaee ${ }^{1,2}$, Behzad Ensan ${ }^{1,2}$

${ }^{1}$ Student Research Committee, Faculty of Medicine, Mashhad University of Medical Sciences, Mashhad, Iran

${ }^{2}$ Department of Neuroscience, Faculty of Medicine, Mashhad University of Medical Sciences, Mashhad, Iran 
The Neuroscience Journal of Shefaye Khatam, 2019; 7(3): P220

Introduction: Multiple sclerosis (MS) is an autoimmune disorder that distinguished by destruction of myelin sheath in the central nervous system. This damage disturbs the ability of parts of the CNS to communicate, resulting in a range of signs and symptoms, including physical, mental, and sometimes psychiatric problems, such as loss of sensitivity or changes in sensation, muscle weakness, blurred vision, very pronounced reflexes, muscle spasms, or difficulty in moving; difficulties with coordination and balance (ataxia); problems with speech or swallowing, visual problems (nystagmus, optic neuritis or double vision), feeling tired, acute or chronic pain, and bladder and bowel difficulties. There are multiple drugs that suppress the progression of MS like: interferon beta-1a, interferon beta-1b, glatiramer acetate, mitoxantrone, dimethyl fumarate, alemtuzumab, and ocrelizumab. Not only are there lots of side effects for people using these drugs but also these kinds of drugs are not cost benefit for economically poor patients. Herbal drugs can be a helpful alternative for curing MS patients. Among several herbal drugs, MS14 is an inexepensive and easy-access one that include: $90 \%$ Penaeus latisculatus, 5\% Apium graveolens and 5\% Hypericum perforatum. This Iranian marine-herbal drug has antiinflammatory and immunomodulatory activities, which shows a significant effect on animal model with Experimental Allergic Encephalomyelitis (EAE). EAE is a demyelinating model that mimics many features of MS. In mice with EAE the level of Interlukine-6 (IL6 ) is higher than normal mice. Several researches has shown that enrichment of mice's food with MS14 is effective in decreasing the level of IL-6. Conclusion: Further research based on animal model of EAE and role of MS14 in patient with MS will be likely useful.

\section{P221}

Smoking and Its Relation with Amyotrophic Lateral Sclerosis: A Systematic Review and Meta-Analysis

Neshat Ebrahimi', Zahra Farjami', 3, Amirhossein Ashnaei $^{2}$, Negar Khodaeinia ${ }^{3}$,Zeinab Sadat Hosseini ${ }^{4}$

${ }^{1}$ Laboratory of Cedars-Sinai Medical Center, Los Angles, California, USA

${ }^{2}$ Department of Modern Sciences and Technologies, Faculty of Medicine, Mashhad University of Medical Sciences, Mashhad, Iran

${ }^{3}$ National Research Center of Genetic Engineering and Biotechnology (NIGEB), Department of Medical Genetics, Tehran, Iran

${ }^{4}$ Student Research Committee, Mashhad Branch, Islamic Azad University, Mashhad, Iran

The Neuroscience Journal of Shefaye Khatam, 2019; 7(3): P221

Introduction: Epidemiologic investigations have provided unpredictable consequences on the relation of cigarette smoking with the prevalence of amyotrophic lateral sclerosis (ALS). In this systematic review and meta-analysis study, we evaluated this association. A systematic search was done using Embase, PubMed, Google Scholar and Science Direct for publications from 2012 to 2017. All retrospective, or prospective observational studies and cross-sectional studies, of amyotrophic lateral sclerosis in relation to smoking exposure were included. Data extraction was done using a standardized data extraction tool in Microsoft Office Excel 2013. All the analyses were done using Stata V.15.0. . 15 investigations reported the relation between ALS and smoking risk in 3 cohort studies and 12 case control studies. The pooled RR (95\% CI) of ALS was 1.46 (0.80 to 1.78$)$ for present vs. nonsmokers and $1.52(0.59$ to 1.63$)$ for ever vs. nonsmokers. The estimated RR of ALS for ever vs. nonsmokers was 0.69 (0.53 to 1.19) in men and 1.36 (1.20 to 2.15$)$ in women. Conclusion: According to the results, smoking increases the risk of ALS especially in women.

\section{P222}

The Role of Long Non-Coding RNA in Regulation and Development of Function in Neurodegenerative Diseases

Fahimeh Nourbakhsh ${ }^{1}$, Samaneh Borooni ${ }^{2}$, Elaheh Tajbakhsh

${ }^{1}$ Department of Pharmacodynamics and Toxicology, Faculty of Pharmacy, Mashhad University of Medical Sciences, Mashhad, Iran

${ }^{2}$ Department of Biology, Nourdanesh Institute of Higher Education, Esfahan, Iran

${ }^{3}$ Departments of Microbiology, Faculty of Basic Sciences, Shahrekord Branch, Islamic Azad University, Shahrekord, Iran

The Neuroscience Journal of Shefaye Khatam, 2019; 7(3): P222

Introduction: Long noncoding RNAs (lncRNAs) also participate in a wide array of subcellular processes; have gained widespread attention in recent years as a potentially new and crucial layer of biological regulation. It was investigated by numerical research that, lncRNAs refer to a group of noncoding RNAs (ncRNAs) more than 200 nucleotides in length in eukaryotic cells IncRNAs including the formation and function of cellular organelles which regulate the expression of the genes related to central nervous system (CNS). Neurodegenerative diseases are associated with multiple clinical manifestations, brain pathologies, and health consequences. Increasing evidence has suggested that lncRNAs are involved in the pathogenesis of neurodegenerative diseases. This review summarizes data on lncRNAs expression in the central nervous system (CNS) and neurodegenerative diseases and focuses on the role of some specific lncRNAs, which may provide new insights into our 
understanding of the etiology and pathophysiology of the neurodegenerative diseases. Several studies have examined the role of lncRNAs and autophagy on cerebral ischemia. For instance, it was reported that lncRNAs H19 as important lncRNAs in CNS promotes autophagy and upregulated in cerebral ischemia and reperfusion (I/R). Conclusion: The evidence from this study highlights that; lncRNAs has important role in the $\mathrm{CNS}$ and is an important factor in neurodegenerative disorders.

\section{P223}

The Posthemorrhagic Neuroprotective Effects of Minocycline by Inducing M2 Microglia Polarization in $\mathrm{ICH}$

\section{Faezeh Sadat Hasheminezhad Hoseini, ${ }^{1,2}$, Leila Amini Noghondar ${ }^{1,2}$, Niluofar Jafari ${ }^{1,2}$}

${ }^{1}$ Student Research Committee, Faculty of Medicine, Mashhad University of Medical Sciences, Mashhad, Iran

${ }^{2}$ Department of Neurology, Faculty of Medicine, Mashhad University of Medical Sciences, Mashhad, Iran

The Neuroscience Journal of Shefaye Khatam, 2019; 7(3): P223

Introduction: Intracerebral hemorrhage (ICH) is the deadliest and lethal subtype of stroke which accounts for $15-20 \%$ of all strokes. ICH is appraised to influence over a million people worldwide each year which is expected to rise substantially as the population ages. Despite the many studies that have been done, there is no authentic treatment for ICH. Therefore, the incessant research for new methods of treating ICH continues, with the hope of ameliorating both the quality and profitability of therapies. Microglial polarization is an innate immune response to $\mathrm{ICH}$. Microglia can be polarized and developed classic M1 (proinflammatory) or M2 (anti-inflammatory) phenotypes by blood components. The M1 phenotype can produce factors that lead to neuroinflammation (which is a major contributor to ICH progression), iron accumulation, and reactive oxygen species (ROS) production that exacerbate brain injury and finally cause brain damage. The M2 microglia can produce anti-inflammatory factors (IL-10, transforming growth factor beta (TGF $\beta)$ ) and promote hematoma clearance through phagocytosis and promotion of angiogenesis. The variant impacts of microglial polarization in $\mathrm{ICH}$ recommend that restraint of M1 microglial activation in the early phase of ICH and promotion of a shift from M1 to M2 phenotype in the late stage could be a challenging therapeutic target for ICH treatment.Current evidence recommends that the antibiotic minocycline (a tetracycline derivative) could be a potential therapeutic drug for neuroinflammatory disease treatment owing to its anti-neuroinflammatory effects. Minocycline is capable of crossing the blood brain barrier which limits inflammation by reducing M1 microglia marker protein
(CD68, CD16) expression and inducing M2 microglial polarization via upregulation of the TrkB/BDNF pathway. Conclusion: Therefore, anti-inflammatory agents such as minoycline which are involved in microglial polarization process are welcomed in the continuing struggle against inflammatory disease.

\section{P224}

Cannabinoids in the Treatment of Epilepsy: Systematic Review and Meta-Analysis

Neda Rabeei, Aghile Heydari, Zeinab Sadat Hosseini

Student Research Committee, Mashhad Branch, Islamic Azad University, Mashhad, Iran

The Neuroscience Journal of Shefaye Khatam, 2019; 7(3): P224

Introduction: Epilepsy is the sudden and abnormal discharge of the nervous tissue of the brain. It is a debilitating and chronic condition and it is the most common disease of the nervous system which it's prevalence is $0.5-1 \%$ in people. Despite Existing treatments $30 \%$ of patients still suffer from epileptic seizures. Cannabinoids seem to have neuroprotective and anti-epileptic effects. Our goal is to systematically review the effect of cannabinoids in the treatment of epilepsy. Systematic search of Embase, PubMed, Science Direct and Google Scholar databases was done between 20132018 with the terms of "Cannabis" OR "Cannabinoids" AND "Epilepsy". We included epidemiological and experimental studies about the medical effects of cannabinoids on the treatment of epilepsy including quasi-experimental, RCTs, non-RCTs, cohort studies, analytical cross-sectional studies and case-control studies. Data extraction was done using a standardized data extraction tool in Microsoft Office Excel 2013. All the analyses were done using Stata V.15.0. . 28 studies were identified including 19 observational studies, and 9 randomized controlled trials (RCTs). The mean age of participants was 18.7 years. Using Cannabinoids $18 \mathrm{mg} / \mathrm{kg}$ per day had more effective role in reducing the seizure than placebo (frequency by $50 \%+\mathrm{RR} 1.53,95 \%$ CI 1.15 to 2.580). The number of Cannabinoids needed for treatment of one person to experience $50 \%+$ reducing the seizure was $6(95 \% \mathrm{CI} 4$ to 12$)$. By investigating the 12 observational studies, $53.2 \%(95 \%$ CI $29.01 \%$ to $64.05 \%$ ) of patients reported $50 \%+$ reducing the seizure. In 7 observational studies $7.7 \%$ (95\% CI $4.8 \%$ to $19.0 \%$ ) were seizure-free. Conclusion: According to the studies were reviewed, RCT investigations are rare in pediatric samples and severe epilepsy syndromes; RCT studies other syndromes and cannabinoids are required.

\section{P225}

\section{PTSD's Promising Treatment}

Negar Khoshghamat, Niloufar Jafari', Seyedeh Motahareh Mirdoosti ${ }^{1}$, Sajad Sahab Negah ${ }^{2}$ 
'Student Research Committee, Faculty of Medicine, Mashhad University of Medical Sciences, Mashhad, Iran

${ }^{2}$ Department of Neuroscience, Mashhad University of Medical Sciences, Mashhad, Iran

The Neuroscience Journal of Shefaye Khatam, 2019; 7(3): P225

Introduction: PTSD is one of the trauma and traumabased disorders with 5-8\% lifetime prevalence in the general population. It occurs following exposure to traumatic events outside the normal range. Main symptoms include re-experiencing, avoidance, numbing, and hyperarousal. Duration of the symptoms must be at least 1 month, along with social and personal dysfunctions. The statics of the 8-year war and enormous earthquakes shows the high level of PTSD vulnerability in Iran. Selective-serotonin reuptake inhibitors and serotonin-norepinephrine reuptake inhibitors are the first-line treatments which can reduce PTSD symptoms as well as associated disorders. Benzodiazepines, Sodium valproate and pregabalin are also suggested for the treatment of PTSD. Previous treatments limitations such as the variety of proposed drugs, safety concerns, adverse side effects, limited efficacy, low tolerability and also controversial results of previous studies led us to design a study to further explore in this issue. Due to the similarity of the pathogenicity mechanisms in depression and PTSD, it is hypothesed that Saffron may have beneficial effects on PTSD. Saffron, the dried stigmas of Crocus sativus L., highly valued agricultural product that largely cultivated in Iran, utilizes as a medicinal herb for various ailments. According the human tendency toward using plant-derived compounds instead of chemical-medicines, special attention must be focused to link the worth of saffron, from basic sciences to patients' bed. Antidepressant and antianxiety effects of saffron have been shown in previous animal studies along with the few clinical trials. Hence it has been suggested as a potential efficient and tolerable treatment with fewer side effects. Conclusion: For this purpose, nanoparticle delivery methods are proved to be more promising, usable and less invasive than any other available methods for the treatment neurological diseases. Although this issue has been examined in animal models with the admissible result, further studies are needed.

\section{P226}

Investigation of p2x7 Receptor Expression in Multiple Sclerosis Patients (Systematic Review)

\section{Elham Safari', Mohammad Reza khojasteh ${ }^{2}$, Elahe Safari $^{1}$}

${ }^{1}$ Student Research Committee, Faculty of Nursery and Midwifery, Mashhad Branch, Islamic Azad University, Mashhad, Iran

${ }^{2}$ Student Research Committee, Faculty of Medicine Mashhad Branch, Islamic Azad University, Mashhad, Iran
The Neuroscience Journal of Shefaye Khatam, 2019; 7(3): P226

Introduction: Multiple sclerosis (MS) is a chronic autoimmune inflammatory disease of the nervous system which is demyelinating neurons.MS usually occurs at the age of 20-50 and is more common in women than men. $\mathrm{P} 2 \mathrm{x} 7$ receptor $(\mathrm{p} 2 \mathrm{x} 7 \mathrm{R})$ which is expressed neurons plays a role in regulation of inflammatory processes. Furthermore studies have shown that $\mathrm{p} 2 \mathrm{x} 7 \mathrm{R}$ is involved in the pathology of MS, so the aim of this study is to checking the expression of $\mathrm{p} 2 \mathrm{x} 7 \mathrm{R}$ in MS patients. This review was performed within articles published at PubMed, Google scholar, Cochrane and SID from 2000 to 2019. The keywords were $\mathrm{p} 2 \mathrm{x} 7$ receptor, multiple sclerosis, expression. By searching this database, 74 articles were found, 36 of them were not related with investigating and 28 of them by reading abstract were removed. All articles chosen from English and Persian articles. Finally, 10 articles were included in our study, in 9 of them reported the expression of $\mathrm{p} 2 \times 7 \mathrm{R}$ in microglial of patients with MS was increased. In more than $95 \%$ of cases, this receptor inhibited the activity of ATP and significantly reduced the ability of phagocytes the neuron cells. In addition, in one of the studies that MS patients were treated with Glatiramer acetate (GA), it was concluded expression of P2X7R in these persons reduced compared to the control group, which showed the effect of GA on inhibiting the P2X7R expression. However, in one study was showed that the expression of P2X7R declined during the acute phase of MS disease. Conclusion: It seems the expression of $\mathrm{p} 2 \mathrm{x} 7 \mathrm{R}$ increase in MS patient and other inflammatory processes, so we can use $\mathrm{p} 2 \times 7 \mathrm{R}$ as a diagnostic and therapeutic method. Therefore, need to be more research done at this topic.

P227

Herbal Therapy: A New Pathway for the Treatment of Multiple Sclerosis

\section{Sara Banaeeyeh, Hosseinzadeh Hosein}

Department of Pharmacodynamics and Toxicology, Faculty of Pharmacy, Mashhad University of Medical Sciences, Mashhad, Iran

The Neuroscience Journal of Shefaye Khatam, 2019; 7(3): P227

Introduction: Multiple sclerosis (MS) is a multifactorial, inflammatory, and neurodegenerative disease of the central nervous system, where environmental factors interact with genetic susceptibility. The aim of the present article was to review the evidence related to medicinal plants in the management of MS symptoms. The literature review was conducted using the electronic databases including Cochrane Library, Web of Science, Pubmed, Scopus and Google Scholar. The key words used as search terms were multiple sclerosis, experimental autoimmune encephalomyelitis, theiler's murine encephalomyelitis virus, cuprizone, lysolecithin, 
medicinal plants, herbal therapy, phytomedicine, and pharmacognosy. No time limitation was considered in this study. Different medicinal plants have shown positive effects on MS including Ginkgo biloba (ginkgo), Zingiber officinale (ginger), Curcuma longa (turmeric), Crocus sativus (saffron), Hypericum perforatum (St John's Wort), Vaccinium macrocarpon (cranberry), Nigella sativa (black seed) and etc. The beneficial effects of herbal medicines in MS disease are occurred by the reduction of CNS inflammation and demyelination. They also show antioxidant and anti-apoptotic properties in neural tissue. Some plant extracts modulate the expression of IL-27, IL-33, enhanced remyelination in the cerebellum and reduce the expression of TGF $\beta$, inhibit the proliferation of $\mathrm{T}$ cells, and the production of inflammatory cytokines as well as selective inhibition of potassium channels of isolated axons. Medicinal plants have demonstrated a new horizon in curing neurodegenerative disorders such as MS. Conclusion: Further studies are needed to disclose the exact mechanisms of action through which medicinal plants exhibit their anti-inflammatory and neuroprotective effects against MS disease.

\section{P228}

\section{A Review of Reducing Vascular Dementia's complications}

\section{Mohammad Saki}

English teaching Department, Hazrat Rasul Akram of Farhangian University, Ahvaz, Iran

The Neuroscience Journal of Shefaye Khatam, 2019; 7(3): P228

Introduction: Vascular dementia (VaD) is recognised as the second most common cause of dementia after Alzheimer's disease (AD), responsible for around $15 \%$ of cases. However, unlike AD, there arecurrently no licensed treatments for $\mathrm{VaD}$. Progress in the field has been difficult due to uncertainties over nosology and diagnostic criteria, controversy over the exact nature of the relationship between cerebrovascular pathology and cognitive impairment, and the lack of identifiable tractable treatment targets. Though there is an established relationship between vascular and degenerative (Alzheimer) pathology, the mechanistic link between the two has yet to be identified. Conclusion: This review critiques some of the key areas and controversies, summarises treatment trials to date and makes suggestions for what progress is needed to advance our understanding of pathogenesis and so maximise opportunities for the search for new and effective management approaches.

\section{P229}

The Study of Neural Stem Cells Improve Cognition Via BDNF in a Transgenic Model of Alzheimer Disease
Sajjad Abbasi', Ali Motamed-sanaye ${ }^{1}$, Mahtab Rahmati', Maryam Moghimian', Masoumeh Fani ${ }^{3}$

${ }^{1}$ Student Research Committee, Gonabad University of Medical Sciences, Gonabad, Iran

${ }^{2}$ Faculty of Medicine, Gonabad University of Medical Sciences, Gonabad, Iran

${ }^{3}$ Department of Anatomical Sciences, Faculty of Medicine، Gonabad University of Medical Sciences, Gonabad, Iran

The Neuroscience Journal of Shefaye Khatam, 2019; 7(3): P229

Introduction: Alzheimer, is a chronic neurodegenerative disease that usually starts slowly and gradually worsens over time. It is the cause of $60-70 \%$ of cases of dementia. Neural stem cell (NSC) transplantation represents an unexplored approach for treating neurodegenerative disorders associated with cognitive decline such as Alzheimer disease (AD). NSC transplantation can improve function by providing missing or defective enzymes or modulating inflammation. used aged triple transgenic mice $(3 \times \mathrm{Tg}-\mathrm{AD})$ that express pathogenic forms of amyloid precursor protein, presenilin, and tau to investigate the effect of neural stem cell transplantation on AD-related neuropathology and cognitive dysfunction. hippocampal neural stem cell transplantation rescues the spatial learning and memory deficits in aged 3xTg$\mathrm{AD}$ mice. Remarkably, cognitive function is improved without altering $A B$ or tau pathology. Instead, the mechanism underlying the improved cognition involves a robust enhancement of hippocampal synaptic density, mediated by brain-derived neurotrophic factor (BDNF). BDNF is decreased within the brains, serum, and CSF of patients with mild cognitive impairment and AD. lossof-function studies show that depletion of NSC-derived BDNF fails to improve cognition or restore hippocampal synaptic density. Conclusion: neural stem cells can ameliorate complex behavioral deficits associated with widespread Alzheimer disease pathology via BDNF. The aim of study is to try Clinical trial of this article.

\section{P230}

\section{Effects of Gluten Free Diet on Multiple Sclerosis}

Zahra Ghodoosi', Marzieh Akbarzadeh ${ }^{2}$, Mohammad Jalali $^{2}$

${ }^{1}$ Faculty of Midwife and Nurse, Shiraz University of Medical Sciences, Shiraz, Iran

${ }^{2}$ Faculty of Nutrition and Food Sciences, Shiraz University of Medical Sciences, Shiraz, Iran

The Neuroscience Journal of Shefaye Khatam, 2019; 7(3): P230

Introduction: Multiple sclerosis (MS) is a chronic autoimmune disorder characterized by demyelinated lesions in the Central Nervous System. Autoimmune disorders such as MS are hypothesized to be kind of atypical celiac disease. Gluten sensitivity or celiac 
disease is a common condition that affects $1-2 \%$ of the population worldwide. Atypical form of celiac can present with extra-intestinal manifestations such as autoimmune diseases. A strict Gluten Free diet is the prominent treatment for celiac disease and also the autoimmune disorders which arises secondary to celiac. The aim of the present study was to systematically review current literature on the impact of gluten free diet on multiple sclerosis. PubMed, Scopus, Embase and Web of Science were searched for clinical trials until 5 March 2019 using the following keywords: "MS", "Multiple Sclerosis", "Autoimmune", "Gluten free", "Free of Gluten", "Gluten Free Diet", "Diet", "GFD" without any filters activated. Fatigue Severity Scale, Mental Quality and Annual Relapse Rate were considered as the primary outcome. In 2 studies, gluten free diet was effective in decreasing patients scores of Fatigue Severity Scale. Also increased patient's quality of life in physical subscales was seen. The duration of diet implementation in these two studies was 3.5 and 12 months. Only in one study, mental quality of life increased with gluten free diet. Another study revealed that after 4.5 years of gluten free diet, lesion activity and also values of Expanded Disability Scale decreased significantly. Gluten free diet might lead to decreased fatigue, and increased quality of life in multiple sclerosis patients. Also it may be effective in decreasing disease activity. Conclusion: Further studies are needed to determine effects of gluten free diet on relapse rate and long term quality of life.

\section{P231}

Caffeine Consumption and Its Association with Parkinson's Disease: Systematic Review and MetaAnalysis

Amirhossein Ashnaei1, Zahra Farjami', Neshat Ebrahimi $^{2}$ Negar Khodaeinia ${ }^{3}$ Zeinab Sadat Hosseini ${ }^{4}$

${ }^{1}$ Department of Modern Sciences and Technologies, Faculty of Medicine, Mashhad University of Medical Sciences, Mashhad, Iran

${ }^{2}$ Laboratory of Cedars-Sinai Medical Center, Los Angles, California, USA

${ }^{3}$ National Research Center of Genetic Engineering and Biotechnology (NIGEB), Department of Medical Genetics, Tehran, Iran

${ }^{4}$ Student Research Committee, Mashhad Branch, Islamic Azad University, Mashhad, Iran

The Neuroscience Journal of Shefaye Khatam, 2019; 7(3): P231

Introduction: According to the recent studies, many scientists indicated the inverse relation between caffeine consumption and the risk of Parkinson's disease (PD). Though, heterogeneity consequences between studies impede a precise estimation of this association. We conducted a systematic review and meta-analysis of epidemiological investigations to estimate the effect of caffeine exposure on PD. Systematic search of Embase, PubMed, Science Direct and Google Scholar databases was done between 2015-2018 with the terms of " Parkinson's disease "AND "Caffeine". We included Cohort, case-control and cross-sectional studies. Data extraction was done using a standardized data extraction tool in Microsoft Office Excel 2013. Heterogeneity was quantified using the I 2 statistic. Of 20 articles, 15 ones had the qualitative criteria for inclusion in our study. The summary RR for 8 cohort studies indicated the relation of caffeine consumption and PD was 0.65 (95\% CI, 0.58$0.72]$, with moderate heterogeneity $(\mathrm{I} 2=27.3 \%)$. For 4 case-control studies, the RR was 0.73 (95\%CI: 0.65-80; $\mathrm{I} 2=6.5 \%$ ), and for 3 cross-sectional studies, the RR was 0.66 (95\%CI: $0.59-0.80 ; \quad \mathrm{I} 2=45.6 \%)$. Conclusion: This study confirms an inverse relation between caffeine consumption and the risk of incidence with PD.

\section{P232}

\section{Hypothalamic Dysfunction; Causes and Solutions}

\section{Shima Sadat Hashemi Madani, Mansoureh Shakeri}

${ }^{1}$ Student Research Committee, Qom University of Medical Sciences, Qom, Iran

${ }^{2}$ Clinical Research Development Center, Qom University of Medical Sciences, Qom, Iran

The Neuroscience Journal of Shefaye Khatam, 2019; 7(3): P232

Introduction: Food provides metabolism, and developing countries are always faced with famine and food shortages. This scourge and deficiency cause many problems in the human body, one of which is a disruption to the functioning of the hypothalamus. In this study, articles published in the PubMed and Congress Library of the Web and the Web of Science were reviewed. Articles related to the purpose of the study were selected. The present article is the result of the study and the abstracts of relevant articles available. As the age grows, the hypothalamus needs support to maintain its normal level of performance .Conclusion: The hypothalamus controls and monitors the autonomic nervous system as a controller and repository for the body's intravenous device, and plays a vital role in certain metabolic processes, and the pituitary gland secretions are regulated by this gland. Thirst, hunger, sleep, emotional behaviors such as anger, temperature and blood pressure, cardiovascular function, etc. are controlled by the hypothalamus. Malnutrition, the normal functioning of many parts of the body, including the hypothalamus, disrupts the body and does not cause harmful nutrients to the body to the hypothalamus. Disruption of the functioning of the hypothalamus leads to depression or abnormal reactions to stress. 


\section{P233}

\section{A Mediated Autonomic Compensatory Response to Afferent Multisensory Integration}

\section{Mohammad Dawood Rahimi ${ }^{1}$, Ruhollah Kashiri ${ }^{2}$}

'Department of Cognitive Psychology, Ferdowsi University of Mashhad, Mashhad, Iran

${ }^{2}$ Mashhad University of Medical Sciences, Mashhad, Iran

The Neuroscience Journal of Shefaye Khatam, 2019; 7(3): P233

Introduction: Migraine is a common and multifactorial complication. Internal energy crisis during multisensory integration in migraine brains is apparent. Different migraine brains respond to different sensory inputs differently: One is sensitive to all sensory inputs, one is to some, and none is insensitive. To do a systematic review - in the present study - the researchers, set the goal on a multi-axial perspective: The hypothalamicpituitary-adrenal (HPA), HPA-Autonomic Compensatory Response (ACR), and HPA-ACR and MitochondrialATP axes. Therefore, in the present study, the researchers reviewed the findings of studies of these axes, their domain interplayers, and multisensory integration-related studies. Their domain interplayers include cortisol, glucocorticoids, reactive oxygen species, oxidative stress, corticotropin releasing factor, brain-derived neurotrophic factor, calcitonin gene-related peptides, and their mediators such as cytokines, interleukins, and mast cells. In the present systemic review, the researchers included English language literature - Google Scholar - published from 2010 to 2019. The researchers further reviewed the keywords such as pathophysiology and underlying mechanism of migraine. After removing duplications, the researchers gathered 417 related articles. Studying these articles, the researchers also found 132 that were cited in these 417 articles. Although there were some contradictions with the relation of these axes, their domain interplayers, and multisensory integration, based on the keywords that were search almost 146 of them emphasized on the relation between migraine and HPA-axis, 13 were on the relation between ACR and migraine, and mitochondrial-ATP related articles were 67. Multisensory integration and migraine related articles were 3 . The other 320 articles were about the abovementioned mediators and domain interplayers. Conclusions: The existence of strong evidence implies that there is a particular relationship between all these axes and mitochondrial or energy crisis which symptomizes before the migraine attacks in the form of fatigue or mood changes. In addition, in the present study, the researchers identified proinflammatory cascade of events that support the idea of migraine as a mediate autonomic compensatory response to afferent multisensory integration. Furthermore, central sensitization and increased activation of neural activities before and during migraine attacks confirm ACR mediation.

\section{P234}

A Review of Therapeutic and Rehabilitation Approaches in People with Congenital Insensitivity to Pain with Anhidrosis

\section{Sahar Sadat Nazm Bojnourdi}

Department of Cognitive Sciences, Cognitive Psychology, Faculty of Psychology and Educational Sciences, Mashhad University of Medical Sciences, Mashhad, Iran

The Neuroscience Journal of Shefaye Khatam, 2019; 7(3): P234

Introduction: The main mechanism of the alarm is the occurrence of pain in the body. Our pain will further alert us to more harmful levels, thus helping us learn from prevention experiences, but how people respond to painful stimuli are different. Lack of sensitivity to pain and reduction of response to painful stimuli and excessive sweating in children can lead us to a point of congenital anesthetic pain (CIPA). The disease is recognized as a type 6 sensory and autonomic neuropathy. The purpose of this study is to assess the therapeutic and rehabilitation strategies of people with CIPA based on research evidence. This study is based on the review of bibliographic and digital resources, Persian journals and medical science databases, and Scopus with CIPA is the key word for rehabilitation and aerobics in 2010-2017. Among the 10 articles found, with emphasis on gene mutation, only 4 articles related Persian and English have been opened and reviewed based on the keywords. Research studies show that medical care and rehabilitation at birth or during postpartum can lead to longer life expectancies in patients. The role of physical rehabilitation along with drug therapy can be effective in improving the clinical symptoms of this disorder. According to available evidence, the use of rehabilitation as a low-cost supplementary therapy significantly improves the quality of life, emotional control, destructive behaviors and musculoskeletal problems in children with CIPA. Conclusion: This study showed that rehabilitation can provide a way for these people to deal with these problems to engage the community.

\section{P235}

Personalized Medicine Approach in Alzheimer's Disease: A Review

\section{Shahriar Kermani}

Student Research Committee, Shahrekord University of Medical Sciences, Shahrekord, Iran

The Neuroscience Journal of Shefaye Khatam, 2019; 7(3): P235

Introduction: Alzheimer's disease (AD), a polygenic and multifactorial disease, is a progressive neurodegenerative disorder and the most common cause of dementia. Amyloid deposition, neurofibrillary 
tangle formation and premature neuronal death are the major neuropathological hallmarks of AD. Personalized medicine is an approach aims to maximize the effectiveness of disease diagnosis, prevention and treatment and to minimize side effects of drugs in different patients with the help of high-throughput OMICS technologies. Although AD Drugs such as Donepezil, Rivastigmine, Galantamine, and Memantine have been developed but their effectiveness is directly related to early-stage AD diagnosis. However, these disease-modifying treatments were not fully satisfactory. Therefore, early-stage diagnosis and prevention of AD are of high importance. So genomics, proteomics and metabolomics studies are essential to identify reliable biomarkers for AD early-stage diagnosis. Genome wide association studies (GWAS), whole genome sequencing (WGS) and whole exome sequencing (WES) showed that Genetic variations in apolipoprotein E (APOE) and proximal genes (PVRL2, TOMM40, and APOC1) are associated with dementia, particularly AD. Epigenetic mechanisms such as DNA methylation and miRNAs may play a central role in the regulation of related gene expression. For instance, miRNA expression can impact the regulation of APP (amyloid beta A4 precursor protein). Also, polymorphisms in the apolipoprotein E and CYP2D6 genes are widely reported to predict therapeutic responses to Donepezil therapy. Conclusion: Taken all together, precision medicine approach in $\mathrm{AD}$ aims to design the best personalized therapeutic intervention by identifying a person's specific pattern of risk factors, related biomarkers and by administering a preventive or therapeutic intervention that is specifically personalized and optimized to the patient.

\section{P236}

\section{The Effect of Expression TWEAK Membrane Protein for Neuroinflammation in Neurological Diseases}

\author{
Samira Fathi', 2, Mohammad Reza Khojasteh ${ }^{1,2}$ \\ ${ }^{1}$ Student Research Committee, Mashhad Branch, Islamic Azad \\ University, Mashhad, Iran \\ ${ }^{2}$ Mashhad Branch Islamic, Azad University, Mashhad, Iran
}

The Neuroscience Journal of Shefaye Khatam, 2019; 7(3): P236

Introduction: The tumor necrosis factor like weak inducer of apoptosis (TWEAK) is a Protein of cell membrane in inflammation, proliferation, angiogenesis and cell death. Although different studies have been conducted around this marker, the exact effect of TWEAK on neuroinflammation continues to be discussed. In this study we aim to evaluate the effect of TWEAK expression on neuroinflammation in neurological disease. In this review we searched PubMed, Cochrane and ISC database during April 2019 with tumor necrosis factor like weak inducer of apoptosis (TWEAK), Nervous system disease and inflammation keywords. We obtained 21, 14 and 6 articles from PubMed, Cochrane and ISC databases respectively. At first step we exclude 21 articles by investigating the title and 13 articles by reviewing the abstracts. 2 articles were duplicated, so at the end we include 5 article in our study. In this study, 5 clinical trials were reviewer. In 4 studies, there is a significant relationship between neuroinflammation and TWEAK expression. So that the neuroinflammation in neurological diseases like MS, increased of TWEAK has been observed. The finding has been shown microglia in subpial cortical lesions and of macrophages in the inflamed meninges expresses TWEAK $(50 \%$ and $80 \%$ 90\%, respectively) indicates. But at the one study they can not see significant difference in the TWEAK protein levels and neurological diseases. Conclusions: According to the results, Evidence suggests TWEAK is involved in the development of neuroinflammation in neurological diseases But Further studies on the expression of inflammatory cytokines can be a good route for future research and Even a way to treat neurological diseases.

\section{P237}

The Study of Epilepsy from a Historical, Diagnostic and Therapeutic Point of View

\section{Zahra Zahadatpour}

Shiraz University of Medical Sciences, Shiraz, Iran

The Neuroscience Journal of Shefaye Khatam, 2019; 7(3): P237

Introduction: Epilepsy is one of the most commonly diagnosed severe neurological disorders in the world involving around 65 million people. It affects $1 \%$ of the population at age 20 and $3 \%$ of the population at age 75. This disease is more common in men than in men, although the overall difference is negligible.the main treatment of epilepsy is medication, antiepileptic drugs have increased in recent years, and are often controlled with an antiepileptic drug for epileptic seizures. A number of patients controlled with a drug have unwanted side effects and may occasionally have epileptic seizures. Approximately $30 \%$ of patients with focal epilepsy and $25 \%$ of patients with primary generalized epilepsy are not well controlled with medication. These patients often use multiple medications and are associated with debilitating epileptic seizures and side effects. Although second-generation drugs are more safe and acceptable, data from these benefits are not yet sufficient. In a major study on current standard drugs, control with two drugs in $40 \%$ of cases can improve, but only in $9 \%$ of cases get rid of epileptic seizures. An analysis of Meta shows that the use of new antiepileptic drugs has been able to reduce the incidence of epileptic seizures, even more, by adding to the previous drug, in $12-29 \%$ of cases. The diagnosis of epilepsy is generally based on the description of seizures and side 
events. Electroencephalography and imaging of the nervous system are also part of the diagnostic operation. Conclusion: It is not always possible to find a specific epileptic syndrome. Long term brain barrier testing with video may also be useful in some cases.

\section{P238}

How Would PLGA Nanoparticles Which Modified with Curcumin Can Help Alzheimer's Disease Therapeutic Intervention?

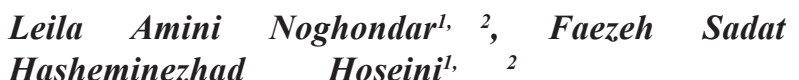

${ }^{1}$ Student Research Committee, Faculty of Medicine, Mashhad University of Medical Sciences, Mashhad, Iran

${ }^{2}$ Department of Neurology, Faculty of Medicine, Mashhad University of Medical Sciences, Mashhad, Iran

The Neuroscience Journal of Shefaye Khatam, 2019; 7(3): P238

Introduction: Alzheimer's disease(AD) is the most common type of dementia, a progressive, neurodegenerative and noncurable which there are no available treatments reverse the progression of the disease. $\mathrm{AD}$ is characterized by neuronal loss caused by amyloid $\beta(\mathrm{A} \beta)$ aggregation, tau hyperphosphorylation and cortical atrophy.AD affects nearly 5.5 million people. Unfortunately, conventional methods like acetylcholinesterase inhibitor drugs, are not so effective owing to restrictive mechanisms imposed at the blood-brain barrier (BBB), poor solubility, and low bioavailability. Also, BBB is a major obstacle facing brain diseases such as multiple sclerosis, brain tumors, and strokes, etc. Engineered nanomaterials are providing interesting biomedical tools potentially able to solve these problems. The important technological advantages of nanoparticles used as drug carriers are high stability, high carrier capacity, the feasibility of incorporation of both hydrophilic and hydrophobic substances, etc. Biodegradable nanoparticles such as PLGA, PLA, chitosan gelatin, etc. Herein PLGA (lactide-co-glycolic acid nanoparticles) remarkably decreased the level of $\mathrm{A} \beta$, reactive oxygen species (ROS), TNF- $\alpha$ and IL- 6 , and enhanced the activities of superoxide dismutase(SOD) and synapse numbers in vitro. Curcumin (Cur) has been proved to have potential use in AD with its antiamyloid, anti-inflammatory, and anti-oxidant properties, etc. But its low solubility and bioavailability hinder its application. Some studies have established that curcumin-encapsulated PLGA nanoparticles (CurPLGA-NPs) potently induce neural stem cells(NSC) proliferation and neuronal differentiation in vitro, as compared to uncoated bulk curcumin. It also hence drug delivery properties of curcumin nano-formulation in the Alzheimer's disease therapeutic interventions. for example: selenium nanoparticles (Se NPs), with B6 peptide and was loaded with Cur (PLGA-PEG-B6/Cur), with rosmarinic acid in form 83-14 MAb-RA-CURPAAM-CL-PLGA NPs and couple nanoparticle with Tet-1 peptide. Conclusion: Due to confirmed effective role of mefenamic acid in $\mathrm{AD}$ treatment we hypothesize that applying mefenamic acid in the curcumin nanoformulation may show promising result.

P239

Curcumin Suppresses Neuroinflammation; a Systematic Review of Preclinical Studies

Seyed Ali Ebrahimi, Mozhgan Yaghoubi, Anahita Ghazaghi

Student Research Committee, Mashhad Branch, Islamic Azad University, Mashhad, Iran

The Neuroscience Journal of Shefaye Khatam, 2019; 7(3): P239

Introduction: Curcumin, the active ingredient of turmeric, has showed anti-inflammatory properties in many disorders including esophagitis, rheumatoid arthritis and ulcerative colitis. However, poor bioavailability of curcumin in the brain has limited its use in neuroinflammatory disorders. Meanwhile, the protective actions of curcumin against some neuroinflammatory disorders have been documented, including Parkinson's disease and depression. The aim of this study was to review in vivo and in vitro studies that investigated the anti-neuroinflammatory properties of curcumin. We performed a systematic review to comprehensively assess the effects of curcumin in experimental models of neuroinflammation. We searched the PubMed, Cochrane and Scopus databases up to April 2019. Search keywords were curcumin, turmeric and neuroinflammation. Ninety-four studies met our inclusion criteria. Irrelevant titles were excluded and eventually 29 articles were reviewed. Only in one of these articles, curcumin did not show any anti-neuroinflammatory effect. Eight articles demonstrated protective effect of curcumin against Alzheimer's disease. In two of these articles, Nano particles were used to increase the poor bioavailability of curcumin, which was successful. Apigenin, showed synergistic effect with curcumin against Alzheimer's disaese. In addition, resveratrol and piperine showed synergistic effects with curcumin. Piperine increased the protective effects of curcumin in models of depression and Parkinson's disease. Furthermore, curcumin inhibited the neuroinflammation in models of Parkinson`s disease (four articles), radiculopathy (two articles), chronic alcohol-induced cognitive deficits (two articles), and epilepsy (one articles). Suppressing NF$\kappa \mathrm{B}$ signalling pathways in activated microglial cells, inhibiting oxidative-nitrosative stress, and protecting axons from degeneration, were the main mechanisms underlying neuroprotective and anti-neuroinflammatory properties of curcumin. Conclusion: current preclinical 
evidences show that curcumin has significant antineuroinflammatory and neuroprotective properties. Novel delivery systems, especially Nano technology, has improved its poor bioavailability. Hence, curcumin can be a potential candidate for developing new antineuroinflammatory drugs. Future clinical studies are required to confirm these results.

\section{P240}

\section{B Cell Therapy for Multiple Sclerosis}

\section{Morteza Saeidi}

Department of Neurology, Mashhad University of Medical Sciences, Mashhad, Iran

The Neuroscience Journal of Shefaye Khatam, 2019; 7(3): P240

Introduction: B lymphocytes and plasma cells play an important role in pathogenesis of multiple sclerosis and induce progression of disease by cytokine secretion, antibody production and antigen presentation. B cell directed therapies have very interested in recent years, B lymphocyte cell depletion therapy offers a compelling and promising new option for multiple sclerosis. $B$ cell activation in multiple sclerosis can induce tumefactive lesions, prominent GAD enhacing lesions, meningeal enhancement, subpial demyelination and... - Monoclonal antibodies targeting CD-20 positive $\mathrm{B}$ cells (such as rituximab), can reduce relapses of multiple sclerosis and also delay disability progression of the disease. Ocrelizumab which is a humanized anti CD-20 monoclonal antibody, reduces the inflammation in relapsing forms of multiple sclerosis and also reduces disability progression in primary progressive ms. Ocrelizumab, now approved for relapsing and primary progressive multiple sclerosis, differ from rituximab in that it has humanized antibody backbone. Other anti-CD20 monoclonal antibodies include ofatumumab (fully humanized with subcutaneous injection), Obinutuzumab (humanized Ig G1) and Ublituximab (an anti-CD20 antibody glycoenginered). Although most disease modifying therapies for MS have traditionally been conceptualized as functioning via $T$ cell based mechanisms, a growing body of data indicates that all have demonstrable effects on $\mathrm{B}$ cells as well, for example beta interferon, glatiramer acetate and fingolimd by shifting B-cell cytokines towards an anti-inflammatory tone. Conclusion: Other mechanism are increasing B-regs, decreasing class II MHC expression and costimulatory molecules on B cells required for Ag presentstion, sequestering B cells in lymphoid organs.

\section{P241}

Evaluation of Correlation Between T Helper Cells and Myasthenia Gravis

Zeinab Boostani ${ }^{1}$, Sepide Cheraghi', Zahra Easy ${ }^{1}$
${ }^{1}$ Student Research Committee, Faculty of Nursery and Midwifery, Mashhad Branch, Islamic Azad University, Mashhad. Iran

${ }^{2}$ Student Research Committee, Faculty of Basic Sciences, Mashhad Branch, Islamic Azad University, Mashhad, Iran

The Neuroscience Journal of Shefaye Khatam, 2019; 7(3): P241

Introduction: Myasthenia gravis (MG) mostly is an autoimmune disease, which the white blood cells start producing antibodies against the site of the nerve to the muscle, for reasons that are often unclear. The prevalence of this disease in different regions of Iran varies from 53 to 74 per 100000 people. T helper cells ( Th cells ) is one of the lymphocyte $\mathrm{T}$ subtypes that accompanies other white blood cells in immune processes including B cells maturation to plasmocytes and memory cells and activation of cytotoxic $\mathrm{T}$ cells and macrophages. The purpose of our study was to evaluate relationship between $\mathrm{T}$ helper cells and myasthenia gravis. We searched Pubmed, Google scholar, Cochrane, SID and ISC databases until December 2018 with myasthenia gravis, T helper cells and acetylcholine receptor keywords. By searching these databases, only 35 articles were found in Pubmed and 87 articles were found in Google scholar.From 122 articles with investigating title we exclude 98 articles and with reading abstract we exclude 16 articles.Finally we include 8 articles in to our study. Our findings demonstrated that certain subsets of $\mathrm{T}$ helper cells are involved in the pathogenesis of $\mathrm{MG}$, including $\mathrm{Th} 1$ and regulatory $\mathrm{T}$ cells ( Treg ). However Th17 cells which recently are identified have an important role in developing MG, it's prognose is still unknown.In one article, the increasing Th17 cells perhaps manifest the severity of disease that is valuable in the diagnosis and therapeutic strategy choice for patients with MG. Conclusion: There is a significant positive correlation between frequency of Th17 cells and anti-acetylcholine receptor antibodies concentration in MG patients. It is proposed to reconstruct the balance of T helper subsets to become a new therapeutic goal for MG. Although more studies are needed in this context.

\section{P242}

The Significant Impact of Apigenin on Different Aspects of Autoimmune Disease

Neda Kasiri', Leila Ahmadi', Mahshid Rahmati', Nahid Eskandari ${ }^{1,2}$

${ }^{1}$ Department of Immunology, Faculty of Medicine, Isfahan University of Medical Sciences, Isfahan, Iran

${ }^{2}$ Applied Physiology Research Center, Isfahan Cardiovascular Research Institute, Department of Physiology, Faculty of Medicine, Isfahan University of Medical Sciences, Isfahan, Iran

The Neuroscience Journal of Shefaye Khatam, 2019; 7(3): P242

Introduction: Systemic autoimmune diseases like Multiple sclerosis and Rheumatoid arthritis are among the highest diseases to diagnose and treat. The current "gold standard" of care for these diseases 
is immunosuppressive drugs which interfere with overall immune responses; their long-term high-dose treatments would expose the patient to opportunistic, life-threatening and long-term malignant infections. Considering the side effects and toxicity of these drug and also the beneficial effects of herbal compounds among their consumers, the professional investigation on the exact mechanism of the plant's major element has grown much attention in the last years. Apigenin as an extracting compound of plants, such as parsley and celery, which has a variety of biological effects, such as anti-inflammatory, anti-cancer and antioxidant effects. This review is intended to summarize the various effects of Apigenin on several autoimmune diseases such as multiple sclerosis which have been worked on so far. The pluralization of the obtained results has revealed Apigenin's effects on pro inflammatory cytokines such as IL-1 $\beta$ and IL6, chemokines such as ICAM-1, immune cells proliferation such as T cells, apoptosis, and various signaling pathways. Conclusion: According to these preclinical findings, we recommend that further robust unbiased studies should be done to use Apigenin as a supplementary or therapeutic element for treating the inflammatory autoimmune disease in the central nervous system or other organs.

\section{P243}

Investigating the Neuroprotective Effect of Oxytocin on Traumatic Brain Injury in Animal Models

\section{Erfan Ghadirzadeh, Ali Siahposht Khachaki, Seyed Pooria Salehi}

Faculty of Medicine, Mazandaran University of Medical Sciences, Sari, Iran

The Neuroscience Journal of Shefaye Khatam, 2019; 7(3): P243

Introduction: Despite all improvements in management and brain stroke care, traumatic injuries are still a major cause of mortality and illness. epidemiology and rate of traumatic brain injuries is significantly higher in men compared with women which shows that this difference might be as a result of sex hormones or estrogen precisely. estrogen regulates synthesis of oxytocin in many organs of body including brain. Estrogen is a normal stress factor which responds to many acute and chronic stimulus and regulates important physiological and biological activities in central nervous system and peripheral nervous system such as facilitating childbirth. Interleukin $\beta 1$ and interleukin $\beta 6$ and interferon $\tau$ and oxytocin regulate synthesis of estrogen receptors. Oxytocin protects tissue from inflammation caused by ischemia or oxidative stress and is compered with GABA signaling (which is a inhibitory neurotransmitter). hyperpolarization of membrane potential caused by GABA type A receptors is related to integral anion channel and therefore infiltration of chloride ions in the direction of electrochemical gradient. Proper balance of transferring inhibitory and stimulatory neurotransmitters help neural network to maintain the normal function of brain but imbalance between them after an ischemic shock results to oversecretion of stimulatory molecules and inhibition of GABA system and down-regulation of GABA type A receptors in plasmic membrane. recent information confirms that oxytocin can also have antiinflammatory and antioxidant effects. Oxytocin can reduce tissue damage in many types of animal injuries. in addition, using a oxytocin receptor antagonist simultaneously can stop antiischaemic effects of oxytocin in heart ischemia in rats. Protective actions of oxytocin can a company with decrease of pro-inflammatory circulating cytokines and filtration of neutrophils in harmed position. These studies provide evidence that oxytocin in regulates GABA type A receptors in their protective effects on traumatic brain injury. Conclusion: however the molecular mechanisms of these protective effects and interactions between oxytocin receptors and GABA type A receptors are still unknown and it has a bigger future than it is right now.

\section{P244}

Spinal Cord Neuropathic Pain and Key Role TLR-4

Roya Ghorbani Ghahfarokhi', Moosa Javdani', Fereshteh Safdari Deh Cheshmeh ${ }^{1}$

${ }^{1}$ Faculty of Veterinary Medicine, Shahrekord University, Shahrekord, Iran

${ }^{2}$ Department of Clinical Sciences, Faculty of Veterinary Medicine, Shahrekord University, Shahrekord, Iran

The Neuroscience Journal of Shefaye Khatam, 2019; 7(3): P244

Introduction: Pain is an unpleasant inward feeling that only the patient can describe. It is a kind of defense mechanism that will make the person aware of the damages however, this unpleasant feeling effects on the individual's life so requires more awareness and treatment. In a type of classification of the pain involves two types of neuropathic pain and physiological pain. Neuropathic pain results from direct damage to the central or peripheral nerves. Damaged nerves send wrong messages to the brain and the brain means these messages as pain. Microglial cells are a type of supporting cells in the central nervous system. These cells are active in response to many external factors and release substances from themselves. The secretion of factors such as chemokines and proinflammatory cytokines likes IL- $1 \beta$, IL-6, TNF- $\alpha$. These substances increase the spontaneous irradiation of neurons that contribute to neuropathic pain. Toll-like receptor4 (TLR-4) is a protein of the toll-like receptor family which is expressed on the microglial cells in the nervous system. Activation of tlr-4 leads to the production of cytokines. Studies have shown the prevention of gene expression TLR-4 causes Reduce significant pain in animal to suffer from spinal cord damage it also reduces the production of inflammatory mediators from microglia cells. The use 
of TLR-4 antagonists, such as (fp-1), was demonstrated in mice with neuropathic pain Due to the inactivation of NF-KB transcription factor and excessive production of TNF- $\alpha$ in the spinal cord injury and the clinical symptoms of neuropathic pain have also decreased. Conclusion: Neuropathic pain is often resistant to existing treatments therefore, the production of ligands for the TLR- 4 can be an important step in relieving pain.

\section{P245}

\section{A Patient with Combined Central and Peripheral Demyelination: A Case Report}

\section{Ali Shahini', Morteza Saeedi ${ }^{2}$}

Student Research Committee, Faculty of Medicine, Mashhad University of Medical Sciences, Mashhad, Iran

${ }^{2}$ Department of Neurology, Faculty of Medicine, Mashhad University of Medical Sciences, Mashhad, Iran

The Neuroscience Journal of Shefaye Khatam, 2019; 7(3): P245

Introduction: Inflammatory demyelinating diseases are, in general, a group of disorders caused by damage of myelin layer because of different factors, for example infections, genetic mutations or immune system's out-of-proportion activation against neuronal myelin, which leads to damage to axons and other neural units. Demyelination causes functional disability for patient that impose notable economic and social burden on countries. Impairment of the neural system are usually limited to the central or peripheral nervous system. Combined central and peripheral demyelination (CCPD) is rather uncommon, and the current information is based on case reports. CCPD is a disease that should not be considered as a simple blend of multiple sclerosis (MS) and chronic inflammatory demyelinating polyneuropathy (CIDP), an adventitious immune mediated disorder of the peripheral nerves system characterized by continuous loss of sensory and motor operations, because most of the patients have homogeneous clinical and laboratory data. A suspected CCPD individual ought to get magnetic resonance imaging (MRI) of CNS and electrophysiological testing to acquire an exact analysis of clinical manifestations. Clinical features are caused by neural demyelination and abnormal nerve conduction, including cranial nerve damage, paralysis, brain and spinal cord lesions, abnormal deep tendon reflexes and optic nerve disturbances. The aim of this research was to investigate clinical and para clinical features of a patient admitted to Mashhad's Ghaem hospital. The patient was 35 years old women suffering from quadriparesis, facial peripheral nerve damage and whole body pain following flu-like symptoms. Multiple abnormal sub cortical and para ventricular patches have been reported in her brain MRI. Conclusion: According to the patient's situation, CCPD has serious and annoying clinical complications for the patient. Therefore, we need to use appropriate diagnostic methods for early diagnosis and minimum complications.
P246

The Evaluation of Foxp3 Gene Expression in Control of $T$ Cells Functions in MS Disease: Systematic Review

\section{Amirhossein Mohajeri Khorasani', Mohammadreza Khojasteh $^{2}$}

${ }^{1}$ Mashhad Branch, Islamic Azad University, Mashhad, Iran

${ }^{2}$ Student Research Committee, Mashhad Branch, Islamic Azad University, Mashhad, Iran

The Neuroscience Journal of Shefaye Khatam, 2019; 7(3): P246

Introduction: Multiple Sclerosis (MS) is an inflammatory autoimmune disease of the central nervous system (CNS), which is more common in young adults. Disorder in tolerance mechanisms such as CD4+ CD25+ T regulatory cells (Treg) may lead to MS disease. In addition, CD8+ T cells may play a supporting role in MS, as they mediate between immunity and tolerance by the acquisition of suppressive activity. Forkhead box p3 (Foxp3) is known to confer suppressive activities in both CD4+ CD25+T cells and CD8 + T cells. We searched PubMed, Google Scholar and Cochrane library from January 2000 until January 2019. 1052 articles were found, which 962 articles were excluded by investigating topics and 81 articles by reading abstract. Finally, 9 articles included in our study. Results demonstrate that not only the Foxp3 mRNA and protein levels in CD4+CD25+ T cells are reduced in MS patients than health controls but also are lower in single-cell level, which result in reducing the suppressive function of $\mathrm{CD} 4+\mathrm{CD} 25+\mathrm{T}$ cells. Moreover, there is a correlation between Foxp3 expression and CBLB mRNA, ITCH mRNA and T helper type 2 cytokine mRNA on the CD4+CD25+ T cells surface. Conversely, the Foxp3 gene is highly expressed in CD8+T cells, involved in tolerance maintenance in MS. Conclusions: The assessment of the Foxp3 gene expression could be one of the options for identification of MS disease since expression variations of this gene are obvious. In addition, The Foxp3 gene expression in CD4+CD25+ T cells can be increased by new methods such as using MicroRNA in order to reduce the complications of this disease. The variations of Foxp3 gene expression in CD8+ T cells in MS need to be studied more, as these cells play an important role in tolerance maintenance in MS.

\section{P247}

The Role of Salt for Autoimmune Disease Exact Multiple Sclerosis: A Systematic Review

\section{Mohammad Ghaderi Zamharir}

Faculty of Medicine, AJA University of Medical Sciences, Tehran, Iran

The Neuroscience Journal of Shefaye Khatam, 2019; 7(3): P247

Introduction: An autoimmune disease is a condition 
arising from an abnormal immune response to a normal body part. In recent decades' scientists have observed a steady rise in the incidence of autoimmune diseases in the world. Since this increase cannot be explained solely by genetic factors, researchers hypothesize that the sharp increase in these diseases is linked to environmental factors. Recent studies provided evidence for an impact of dietary habits on the immune status and related disorders. Salt (sodium chloride; $\mathrm{NaCl}$ ) is one component prevalent in processed food frequently consumed. Studying the mechanisms behind the effects of sodium chloride on immune cells like T helper (TH) 17 cells and its implication as a risk factor for several diseases is a main goal. This essay was a systematic review of English articles published in PubMed, Nature, Semantic scholar and Research gate since 2010. Being up to date, matching with keywords and accessing the full text were incoming metrics. Interleukin(IL)-17 producing T helper cells (Th17 cells) plays a pivotal role in autoimmune diseases exactly multiple sclerosis(MS). Researchers show that increased salt concentrations found locally under physiological conditions in vivo dramatically boost the induction of murine and human Th17 cells. Most researches demonstrated that excessive salt enhances the differentiation of Th17 cells, inducing a highly pathogenic phenotype that aggravates experimental neuroinflammation. First human studies revealed an association of increased MS disease activity with elevated salt consumption, while more recent epidemiology studies in larger cohorts suggest no correlation between salt intake and MS. Recent data from long-term studies with volunteers subjected to constant sodium intake have shown that urine $\mathrm{Na}+$ concentrations oscillate between day to day measurements. However, it is known that ordinary urinary sodium analyses and nutritional questionnaires do not necessarily correspond to the actual sodium load and more sophisticated analyses are needed. Conclusions: Therefore, only further studies under less extreme conditions can show the extent to which increased salt intake actually contributes to the development of autoimmune diseases. Although more research is needed on the mechanisms underlying the impact of lifestyle on MS, studying the combined lifestyle factors simultaneously would help to enhance our understanding of the multifactorial impact on MS etiology.

\section{P248}

\section{The Cognition Impairments in Alzheimer's Disease}

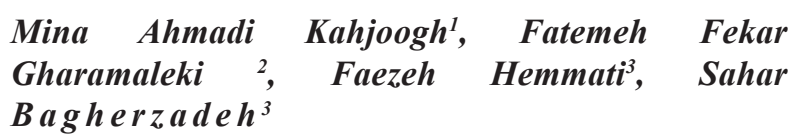

${ }^{1}$ Department of Occupational Therapy, Faculty of Rehabilitation, Tabriz University of Medical Sciences, Tabriz, Iran

${ }^{2}$ Department of Speech Therapy, Faculty of Rehabilitation, Tabriz University of Medical Sciences, Tabriz, Iran
${ }^{3}$ Faculty of Rehabilitation, Tabriz University of Medical Sciences, Tabriz, Iran

The Neuroscience Journal of Shefaye Khatam, 2019; 7(3): P248

Introduction: Alzheimer's disease (AD) is diagnosed when it has reached the stage at which cognitive (i.e., episodic memory impairment) and neuropsychiatric symptoms interfere with social functioning or activities of daily living. The dementia diagnosis is strongly based on clinical judgment, for which appropriate assessment instruments are of vital importance Diagnosis and treatment of AD at early stage is important issue in rehabilitation fields and speech and language pathology. We conducted a search in PMC, Web of Science, PubMed, Scopus, Medline, SID, Google Scholar and Ovid databases. The present information is available reviewing the articles from 1996 to 2019 through the search of the resources. Cognition analyses have already been used in patients with dementia. The disorder impairs the basic level of cognition skills as well as higher level. Therefore, AD patients is characterized by cognition problems such as memory, language and visuspatial functions. They have attention deficits include selective and sustain and divided attention problems. AD assessment is generally based on cognition and language ability. Speech therapy is one of the most important rehabilitation field for AD patients, which can help regain important cognitive and communication functions. An SLP will also teach to AD patients how to adapted and relearn speech and language, also can help AD patients regain cognitive function and memory, which can improve Activity of Daily Living. Speech therapy methods can decrease brain degeneration rate in Alzheimer's Disease. Conclusion: The aim of therapy is to recover as much of speech as possible and/or find alternative ways of communicating.

\section{P249}

The Effect of Traditional Medicine on Epilepsy Treatment

Ali Honarmand, Rasul Saberi, Mohammad Salari, Mohadese Feyzmohammadi

Biology Department, Faculty of Basic Sciences, Islamic Azad University, Mashhad, Iran

The Neuroscience Journal of Shefaye Khatam, 2019; 7(3): P249

Introduction: Epilepsy is one of the chronic neurological disorders. Today, more than 70 million people in the world are suffering from epilepsy using modern Western medicine. However, about $30-40 \%$ of patients are resistant to drugs and suffer from complications. A search in two PubMed and Google Scholar databases was conducted in the last five years, with a total of 84 articles found. Regarding their relationship with the subject, 23 articles were examined. The results of this study were from 
traditional Iranian and Chinese medicine. For example, by reviewing some of the most important botanical sources in Iran, such as Canon, al-Hawi, al-Abniah 'an Haqaeq al Adwia, Tuhfat al-Mu'minin, and Makhzan ul-Adwia, several herbs as herbal remedies for the treatment of epilepsy is identified. Traditional Chinese Medicine (TCM), which has been used centuries ago for the prevention and even treatment of epilepsy in China. The compounds obtained from the plants have antiinflammatory, antiseptic, and anticoagulant properties that have been shown to be positive in in vivo and in vitro experiments. In these compounds, the presence of Flavonoids, Furanocoumarins and Terpenoides is observed, which causes anticonvulsant effects in these plants. Gastrodin, Asarone, Rhynchophylline, Curcumin, etc are Chinese medicine monomers that, by regulating neuronal receptors, expressing inflammatory factors, express the apoptotic protein with anti-epileptic effects. Conclusion: Therefore, due to the severe side effects of the use of commonly used chemical drugs, as well as the efficacy of traditional medicine and herbs in epilepsy treatment, medicinal herbs can be the basis for discovering new antiepileptic drugs.

\section{P250}

\section{Physical Exercise as an Effective Factor in Alzheimer} Disease

Negar Khodaeinia', Zahra Farjami, ${ }^{1,2}$, Amirhossein Ashnaei $^{2}$, Neshat Ebrahimi ${ }^{3}$, Zeinab Sadat Hosseini ${ }^{4}$

${ }^{1}$ National Research Center of Genetic Engineering and Biotechnology (NIGEB), Department of Medical Genetics, Tehran, Iran

${ }^{2}$ Department of Modern Sciences and Technologies, Faculty of Medicine, Mashhad University of Medical Sciences, Mashhad, Iran

${ }^{3}$ Laboratory of Cedars-Sinai Medical Center, Los Angles, California, USA

${ }^{4}$ Student Research Committee, Mashhad Branch, Islamic Azad University, Mashhad, Iran

The Neuroscience Journal of Shefaye Khatam, 2019; 7(3): P250

Introduction: Alzheimer's disease (AD) is a progressive disease that destroys memory and other important mental activities. Scientists have found that remaining relatively active can lead to better brain activities in those at risk of developing AD. In some Meta-analyses of prospective investigations, a significantly reduced risk of dementia related to midlife exercise have been proven. Most studies have been performed on animal models about the effects of exercise on brain $\beta$-amyloid deposition, showed that the level of amyloid plaques are reducing significantly. In another study about the serum BDNF levels and exercise have recognized major transient increases of circulating BDNF with shortterm aerobic exercise. This information recommend that aerobic exercise is related to a decreased risk of cognitive impairment and dementia. A convergence of data from both human and animal studies proposes that aerobic exercise may reduce development of neurodegenerative processes and age-association loss of synapses. Conclusion: This may occur by a direct effect on neurodegenerative disease procedures or simplification of neuroprotective neurotrophic factors.

\section{P251}

Evaluating the Effects of Functional Electrical Stimulation on the Rehabilitation of Patients with Multiple Sclerosis: A Systematic Review

\section{Sara Saremi Nia ${ }^{1,2}$, Mohammad Reza Khojasteh ${ }^{1,2}$ \\ ${ }^{1}$ Department of Mashhad Neuroscience Research, Mashhad Branch, Islamic Azad University, Mashhad, Iran \\ ${ }^{2}$ Student Research Committee, Mashhad Branch, Islamic Azad University, Mashhad, Iran}

The Neuroscience Journal of Shefaye Khatam, 2019; 7(3): P251

Introduction: Functional electrical stimulation (FES) is a technology that can be used on paralyzed muscles to allow them to move. It has been shown to functionally restore and rehabilitate individuals with movement disorders, such as stroke, multiple sclerosis (MS) and traumatic brain injury. We searched Pubmed database until March 2019 with functional electrical stimulation, multiple sclerosis and rehabilitation keywords. By reading the titles, sixty-seven articles out of a total of 96 items were eliminated. Finally, we started the study with 29 remained articles that had relative abstracts. Twenty-nine relevant studies were reviewed which five were pilot, two were feasibility and one was preliminary studies but other studies are not categorized in these groups. All of these showed various benefits of using FES to rehabilitate patients and reduce some effects of MS. Twelve articles showed gait improvements and in seven articles foot-drop was studied. In Other articles, effects of FES on reducing chronic constipation of people with multiple sclerosis (pwMS), improvements in their activities of daily living, cardiorespiratory and physiological cost of gait and muscle metabolism were also mentioned. Conclusion: The evidence presented in this review suggests that FES has a positive effect on the rehabilitation of pwMS. There was an absence of the information about the exact time required for getting access to the final result. Also the cost and accessibility of using the device referring to the benefits of the result is not obvious enough. It seems that further studies should increase the number of participants and also find other aspects and effects of using FES on MS.

\section{P252}

Oral and Craniofacial Manifestations of Multiple Sclerosis

Zahra Ranjbar, Azita Azad 
Department of Oral and Maxillofacial Medicine, Faculty of Dentistry, Shiraz University of Medical Sciences, Shiraz, Iran

The Neuroscience Journal of Shefaye Khatam, 2019; 7(3): P252

Several neurologic disorders and painful conditions happen in MS and orofacial region may be affected by these disturbances. The disease and the drugs used to treat or control it can affect the oral status and oral health care. One research evaluates 500 patients with MS and a frequency of $88.6 \%$ for craniofacial manifestations was reported in this research. So multidisciplinary team is needed and dentist plays a significant role in the healthcare of these patients. Orofacial region can show initial signs and symptoms of neurologic disturbances. Intermittent unilateral facial numbness or pain (neuropathic or neuralgic pain), facial palsy $(19 \%)$ or spasm $(6-11 \%)$ and dysphagia $(26.6 \%)$ are the most common symptoms in orofacial region. Also, myofascial and neck pain has been seen and may due to a high frequency of temporomandibular disorders $(58.2 \%)$ in MS patients. Trigeminal neuralgia (TN) is the first manifestation of MS In $0.3 \%$ of cases and it is observed in $7.9 \%$ of MS patients. TN frequently affects the maxillary and mandibular division and rarely $(<5 \%)$ affects the first branch of the trigeminal nerve. In addition, some case reports show other orofacial problems, like glossopharyngeal neuralgia $(0.5 \%)$, paroxysmal cluster-like pain and trigeminal autonomic cephalgia. furthermore headache can be observed in MS patients with a frequency of 13 to $64 \%$. Also drugs taken by MS patients may lead to oral side effects like opportunistic infection, xerostomia, mucositis and gingival hyperplasia. In addition, orofacial pain may affect quality of life and the ability to do daily activities, such as effective oral hygiene. In conclusion, oral health care providers may faced with initial signs and symptoms in undiagnosed patients and have an important role in the management of orofacial problems of MS.

\section{P253}

Bevacizumab in Treatment of Glioblastoma: Recent Progress and Challenges

\section{Nima Zafari, , Parsa Bathhaee ${ }^{1,2}$, Behzad Ensan ${ }^{1,}$ 2, Mohammad Bagher Soltanian ${ }^{1,2}$, Mahshid Safari Ghalezou $^{1,2}$}

'Student Research Committee, Faculty of Medicine, Mashhad University of Medical Sciences, Mashhad, Iran

${ }^{2}$ Department of Neurology, Faculty of Medicine, Mashhad University of Medical Sciences, Mashhad, Iran

The Neuroscience Journal of Shefaye Khatam, 2019; 7(3): P253

Introduction: Grade IV glioma known as Glioblastoma multiforme (GBM); is the most malignant primary brain tumor in adults. Features of GBM include invasion, aggression and angiogenesis. GBM patients have median survival time of 14.6 months approximately, due to tumor recurrence. Common treatment is surgery followed by chemo radiation. Bevacizumab (BEV) is a recombinant humanized monoclonal antibody that targets vascular endothelial growth factor (VEGF). It has been studied in both recurrent and newly diagnosed GBM but, it is approved by U.S. Food and Drug Administration only for improving recurrent GBM patients. Main function of $\mathrm{BEV}$ is its antiangiogenic effect. A retrospective cohort study have indicated that use of angiotensin system inhibitors (ASI) or calcium antagonists with BEV therapy could be effective for recurrent GBM treatment. BEV with concomitant Temozolomide and radiotherapy was shown to prolong progression-free survival in also newly diagnosed GBM patients. Available documents signify that BEV treatment increased glioma invasion and made them more resistant so because of upregulation of metalloproteinase activity, a crucial regulator of GBM invasion, the tumor relapse after BEV therapy is represented as a non-enhancing infiltrative phenotype in the radiographic imaging. Recent studies signified that $\delta$-catenin is resulting BEV-induced glioma cell invasions; some others showed that stimulation of protein kinase B (AKT), a serine-threonine kinase is also implicated in it. Available documents signify that the combination of $\mathrm{BEV}$ with an integrin inhibitor (Cilengitide) suppressed the integrin-mediated cell adhesion pathway as an underlying mechanism of its anti-invasive effect so it exerted a significant anti-invasive effect. Conclusion: These findings like therapeutic targeting of AKT signal and $\delta$-catenin or use of cilengitide could be the subject of further researches for new methods of treatment.

\section{P254}

Reducing the Number of Deaths from Stroke: Emergency Care and Functioning

\section{Ehsan Sharifipoor ${ }^{1}$, Hoorolnesa Ameli ${ }^{2}$, Shima Sadat} Hashemi Madani ${ }^{3}$

${ }^{1}$ Department of Neurology, Qom University of Medical Sciences, Qom, Iran

${ }^{2}$ Department of Emergency Medicine, Qom University of Medical Scineces, Qom, Iran

${ }^{3}$ Qom University of Medical Scineces, Qom, Iran

The Neuroscience Journal of Shefaye Khatam, 2019; 7(3): P254

Introduction: Stroke is one of the leading causes of disability in the world. Despite all the improvements in the treatment of ischemic stroke, the proportion of patients receiving innovative therapies is very high, but the results are very poor. Cerebral vascular obstruction is very important during the first hours and up to 24 hours. The information is very important since the emergence of strategies for the care and treatment of ischemic stroke and haemorrhagic attack. This study highlights the need for population and health care. The diagnosis of stroke symptoms and immediate measures play an 
important role in improving the performance of rescue workers and all emergency medical personnel to reduce damage. Always talk about the dangers of a disease: It may be wise to minimize the risk of diseases such as stroke because it aims to provide scientific solutions to reduce the number of attacks throughout the country. Conclusion: With confidence, with the gap between the theory of knowledge and clinical practice narrowing, emergency solutions will be more effective.

\section{P255}

Tachykinins: The Key Players in Neurodegenerative Diseases

\section{Seyed Isaac Hashemy ${ }^{1,2}$}

${ }^{1}$ Department of Clinical Biochemistry, Faculty of Medicine, Mashhad University of Medical Sciences, Mashhad, Iran

${ }^{2}$ Surgical Oncology Research Center, Mashhad University of Medical Sciences, Mashhad, Iran

The Neuroscience Journal of Shefaye Khatam, 2019; 7(3): P255

Introduction: Tachykinins (TKs) are a family of neuropeptides widely distributed in the whole body, especially in the nervous system. Several pieces of evidence suggest that TKs could exhibit both neuroprotective and neurodegenerative properties in the central nervous system (CNS) and spinal cord. It is also now well established from a variety of studies that substance $\mathrm{P}$ (SP), as a pioneering neuropeptide of the TK family, is involved in the pathogenesis of neurodegenerative disorders (NDs), such as Alzheimer disease, Multiple Sclerosis, Parkinson's disease, Huntington's disease, and Amyotrophic lateral sclerosis. However, there is a huge amount of conflicting information about the level of SP in NDs that propose SP and its receptors might be prognostic or diagnostic factors for NDs. Conclusion: The present review article summarizes the roles of TKs in common neurodegenerative disorders.

\section{P256}

\section{Autistic Children; New Educational Techniques}

Samineh Saghafinya ${ }^{1}$, Shima Sadat Hashemi Madani ${ }^{2}$

${ }^{1}$ Iran University of Medical Sciences, Tehran, Iran

${ }^{2}$ Student Research Committee, Qom University of Medical Sciences, Qom, Iran

The Neuroscience Journal of Shefaye Khatam, 2019; 7(3): P256

Introduction: Autism or autism is a disease of developmental disorder in the central nervous system that is affected by genetic factors and is stimulated by environmental factors. This disruption of growth, in social events, brings with it many problems throughout life. In addition to timely diagnosis and treatment of this disease, it is necessary to design new and different diagnostic and therapeutic methods. Search was conducted in Pubmed / Medline databases. 20 studies aimed at familiarizing with the new methods of educational and therapeutic use of autistic children were studied and content was similar to the present study. It was included in this article. Improving the education and treatment of children with autism spectrum is essential for improving their social performance in the community. Conclusion: In patients with autism, there are behavioral, cognitive and early interventional disorders. Because this neuropathic disorder occurs first in childhood. Therefore, teaching and learning, especially with new methods, is very important in children with autism spectrum. The design of educational, therapeutic, recreational and computer simulators, in accordance with the physical and mental conditions of children, plays a significant role in the improvement of the disease. We also believe that research is needed from theory to implementation and application in the community and to reduce the many problems of autistic children.

\section{P257}

The Effects of Ethanol on the Nervous System in Adolescence and Youth: A Review Article

\section{Hossein Bakhtiari', Mahla Rezaei ${ }^{1}$, Farimah Beheshti}

${ }^{1}$ Student Research Committee, Torbat Heydariyeh University of Medical Sciences, Torbat Heydariyeh, Iran

${ }^{2}$ Neuroscience Research Center, Torbat Heydariyeh University of Medical Sciences, Torbat Heydariyeh, Iran

The Neuroscience Journal of Shefaye Khatam, 2019; 7(3): P257

Introduction: Adolescent brain development seems to be important for the maturation of brain structures and behavior. Intermittent binge ethanol drinking is common among adolescents, and this type of drinking can induce brain damage. We searched the literature available in Scopus, PubMed, Google scholar and Science Direct databases for English articles published until 1998. For this purpose, we used 3 keywords for ethanol, 4 for animal models and 8 for nervous system disorders. This review article summarized a variety of in vitro and in vivo studies in order to find out the effects of ethanol in nervous system. Based on the findings of this review study, persistent consumption ethanol, can induced memory abnormalities in adolescents. In addition, repeated consumption of ethanol leads to a deficiency of thiamine intake, an excess of $\beta$-amyloid peptide and resulting in oxidative stress in the brain. Even the results showed that brief contact with pregnant women in the second quarter with ethanol would have a negative effect on the neonatal performance of the offspring. Some other studies have shown that they may have a relationship between acetylcholinesterase, apoptosis and neuro-inflammation of neurons and nerves. Conclusion: These findings indicate that binge 
pattern exposure to ethanol during adolescence induces brain damage by inflammatory processes and causes long-lasting neurobehavioural consequences.

\section{P258}

Investigating the Potential Impact of Variables in non-Translocational Regions of Key Genes in Parkinson's Disease and Their Relationship with Disease Progression

Setareh Samizadeh', Masoumeh Masah', 3, Alireza Nasr Isfahani', Samira Rahimi Rad", ${ }^{1}$, Mansoureh Azade $^{2,4}$

${ }^{1}$ Islamic Azad University, Najafabad, Iran

${ }^{2}$ Department of Biochemistry, Islamic Azad University, Najafabad, Iran

${ }^{3}$ Researcher Biotechnology Institute, Department of Biotechnology, Nonprofit University of Ashrafi Esfahani, Esfahan, Iran

${ }^{4}$ Zist Fanavary Novin, Biotechnology Institute, Esfahan, Iran

${ }^{5}$ Parand Brnch, Islamic Azad University, Parand, Iran

The Neuroscience Journal of Shefaye Khatam, 2019; 7(3): P258

Introduction: Parkinson's disease (PD) is a disturbance of progressive neurodegenerative disorder in the nervous system qualified by poly faceted and varied motor- and non-motor symptoms and possible interaction of genetic and environmental risk factor. $\mathrm{PD}$ is the second most commonly reported disease is the CNS. One of the possible cause is single-nucleotide polymorphisms (SNP) in the untranslated region (UTR) of active genes in CNS. The literature review was used to collecting the PD-related most susceptible genes. Then, using miRNASNP database, the miRNA-related SNPs located on the 3'UTR of genes were identified. Finally, the pathway enrichment analysis was performed by the KEGG database. Our results demonstrated that SNCA, LRRK2, and PINK1 are critical genes in PD progression, in which on their 3'UTR there are some high-frequency polymorphisms located within the miRNAs target site. These variants may result in an expression blockage of mentioned genes during PD. The pathway analysis resulted to found that miRNAs in genes (SNCA rs17016074 G/A with miRNA: hsa-miR499a-3p, LRRK2 rs3886747 C/T with miRNA: hsamiR-551b-5p/3p, PINK1 rs1043502 T/G with miRNA: hsa-miR-3126-5p/3p) have interfering effects on same of the cancers and hepatitis genes. Conclusion: Upon the fact that any changes in SNP alleles cause mutations in the vital genes for the normal functioning of the nervous system, it can be stated that these SNPs may play role in development and progression of PD. These results may open the new route to develop molecular controlling of Cancer, Hepatitis and PD in future.
Evaluation of the Role of Autonomic Nervous System (ANS) and Sympathetic Nervous System Stimulants

\author{
Shima Sadat Hashemi Madani ${ }^{1,2}$, Mansoureh \\ Shakeri ${ }^{1,} \quad 2$ \\ ${ }^{1}$ Student Research Committee, Qom University of Medical \\ Sciences, Qom, Iran. \\ ${ }^{2}$ Clinical Research Development Center, Qom University of \\ Medical Sciences, Qom, Iran
}

The Neuroscience Journal of Shefaye Khatam, 2019; 7(3): P259

Introduction: The autonomic nervous system (ANS), which is responsible for unconscious activities of the body, is considered part of the environmental nervous system. Involuntary actions, such as heart rate or narrowing of the blood vessels, are done by its motor fibers, which go to smooth muscle, heart muscle and glands. Finally, the control of the cardiovascular activity set; the digestive functions and tail adjustment are the responsibility of the system. The search was conducted in Pubmed / Medline databases. 60 studies were conducted to evaluate the role of the autonomic nervous system (ANS) and the sympathetic nervous system stimulating factors, which were similar in content to the present study and were included in this paper. Stress, fear, excitement, pain and cold cause the sympathetic nervous system to stimulate, and blood pressure, heart disease, respiratory tract and swallowing can disrupt the system. Conclusion: Sympathetic nervous system is known as an automatic device. Factors such as excitement, stress, fear, pain and cold affects the sympathetic nervous system. Increasing contractility and heart rate, blood pressure, blood vessel stenosis, sweating and blood glucose, increased levels of glycolysis, and decreased digestive function, central adrenal gland stimulation, bronchial enlargement, pupilitis, etc. Cases are effective.

P260

Neuromyelitis Optica Spectrum Disorders Coinciding with Systemic Autoimmune Diseases: a Case Report

\section{Ghazaleh Pourali', Morteza Saeedi ${ }^{2}$}

${ }^{1}$ Student Research Committee, Faculty of Medicine, Mashhad University of Medical Sciences, Mashhad, Iran

${ }^{2}$ Department of Neurology, Faculty of Medicine, Mashhad University of Medical Sciences, Mashhad, Iran

The Neuroscience Journal of Shefaye Khatam, 2019; 7(3): P260

Introduction: Neuromyelitis optica (NMO) and neuromyelitis optica spectrum disorders (NMOSD) are a group of inflammatory demyelinating diseases affecting the central nervous system (CNS) described by immune-mediated axonal damage to optic nerves and spinal cord. Previously, they were considered a multiple sclerosis variant, but based on unique immunologic pathophysiology, they are now recognized as an 
independent disease entity. The exact causes of NMOSD are unclear, but the role of anti-aquaporin-4 autoantibody(AQP4-Ab) in the pathogenesis of NMOSD has been indicated in previous studies. In recent years, there has been growing information of NMOSD association with other systemic autoimmune diseases, such as myasthenia gravis (MG), systemic lupus erythematosus (SLE), autoimmune thyroid diseases, and Sjögren's syndrome bringing up ideas of shared autoimmune pathophysiology and genetic susceptibility. Recognizing these comorbidities is without a doubt necessary for appropriate treatment and prevention of neurologic and systemic complications over time. In this article, we describe a case of NMOSD presenting with concurrent myasthenia gravis. The patient was a young woman of about 30 years of age referred to Mashhad's Ghaem hospital with a history of upper and lower extremity weakness, bilateral fatigable ptosis and blurred vision. Clinical features and para-clinical investigations indicated coexistence of NMOSD and myasthenia gravis. Conclusion: According to this study and other reports, clinicians should carefully investigate atypical systemic or laboratory signs in all patients with NMOSD to rule out other related comorbidities because these comorbidities may affect the treatment plans and thus may improve the patients' outcome.

\section{P261}

\section{MiR326: A Potent Biomarker in Early Diagnosis and Treatment of Multiple Sclerosis}

\section{Sara Yousefi Tabari $^{1}$, Negar Gholampoor ${ }^{2,}{ }^{3}$, Motahareh Mirdoosti ${ }^{2,}{ }^{3}$ \\ ${ }^{1}$ Student Research Committee, Faculty of Medicine, North Khorasan University of Medical Sciences, Bojnurd, Iran \\ ${ }^{2}$ Student Research Committee, Faculty of Medicine, Mashhad University of Medical Sciences, Mashhad, Iran \\ ${ }^{3}$ Department of Neurosciences, Faculty of Medicine, Mashhad University of Medical Sciences, Mashhad, Iran}

The Neuroscience Journal of Shefaye Khatam, 2019; 7(3): P261

Introduction: Multiple sclerosis(MS), the most common demyelinating disease, is a chronic neurodegenerative disorder of the central nervous system (CNS). It is associated with various degrees of disability such as mobility problems and encounters the society with high costs of treatment. According to statistics, about 2.3 million people are suffering from MS, globally. Early diagnosis and treatment of MS is proved to bring significant benefits for patients. The etiology of this illness is complicated, but it is believed that abnormal inflammatory responses, especially by certain types of $\mathrm{T}$ cells initiate tissue damage in MS. MicroRNAs (MiRNAs), are short, noncoding RNAs which play key roles in cellular regulation and mRNA expression, have been established evidence to be promising candidates for treatment, diagnosis and prognosis of different types of diseases. There is evidence that expression of special kinds of miRNAs such as miR326 increases in exosomes derived from conventional $\mathrm{T}$ cells (Tconv) which mediates underlying mechanisms in patients with MS. MiR326 was formerly found to have a remarkable function in immunopathogenesis of MS due to its role in T-helper 17 (TH17), a unique CD4+ T-cell subset characterized by production of interleukin-17 (IL17), differentiation and maturation. These changes in miR326 levels are also easily detectible in patient's blood; as a result, it can be used as a reliable biomarker in early detection of MS. Taken together, we assume that by meticulous observation of miR326 level in blood, we can detect MS in early stages. Conclusion: Furthermore, extracellular vesicles that carry suppressors which target miR326 expression in Tconv can be used as a novel way to restrict the MS progression.

\section{P262}

The Effect of Stem Cell Transplantation in the Treatment of Parkinson's

\section{Rasul Saberi, Ali Honarmand, Mohammad Salari}

Biology Department, Faculty of Basic Science, Islamic Azad University, Mashhad, Iran

The Neuroscience Journal of Shefaye Khatam, 2019; 7(3): P262

Introduction: Parkinson's disease (PD) is a neurogenic disorder that causes movement disorders in humans. Standard therapies and former therapies, such as Levodopa prescription, can not prevent the development of the disease and control long-term motor symptoms. However, cell therapy can have a potential impact on the disease. With the search in this review study on Google scholar and Pubmed databases, 77 papers agreed with entry filters, which were reviewed by title and abstracts of articles from 21 articles between 2014 and 2019 and targeted studies. The results showed that due to the ability of the brain to respond to disorders and restore the population of damaged neurons in the adult mammalian brain, Stem / progenitor cells have a potential role in restoring CNS damage. However, the transplantation of adipose-derived stem cells (ADSCs), Mesenchymal stem cells (MSCs), Human dental pulp stem cells (HDPSCs), Embryonic Stem Cells (ESCs) and Induced Pluripotent Stem Cells (IPSCs) in animal and human models Due to their ability to activate microglia, damaged tissue germination, migration capacity and having anti-inflammatory, immunomodulatory and neurodegenerative properties, they can be effective in slowing the PD process. Also, RHO kinase inhibitors (Rock), due to their effects on stabilizing axons of damaged neurons, protecting neuron death, and the backwardness of axon and biological nanoparticles, also increase the survival of stem cell transplantation and 
improve the delivery of cell to brain. They can be useful before and after transplantation of stem cells. In these studies, stem cell transplantation can slow down or stop Parkinson's disease. Conclusion: Also, RHO kinase inhibitors and biological nanoparticles can help with the treatment of this disease. Of course, timely diagnosis is very effective in the recovery process.

\section{P263}

Mobile Phone, the Effects of Electromagnetic Waves on the Brain and Memory Impairment

Ehsan Sharifipoor ${ }^{1}$, Mohammad Reza Zaheri ${ }^{2}$ Mohamad Shahidi', Samineh Saghafinya ${ }^{3}$, Shima Sadat Hashemi Madani ${ }^{2}$

${ }^{1}$ Department of Neurology, Qom University of Medical Sciences, Qom, Iran

${ }^{2}$ Department of Emergency Medicine, Qom University of Medical Scineces, Qom, Iran

3University of Medical Sciences, Tehran, Iran

The Neuroscience Journal of Shefaye Khatam, 2019; 7(3): P263

Introduction: The human brain works with electromagnetic waves and is different from other neurons and neurons (neurons) and geometric shapes. Environmental information, magnetic waves and chemical patterns, converge to electromagnetic waves as soon as they arrive at the first receptor of the brain. Memory, which is a network and works with a kind of active protein (microtubule), inside the nerve cells; and waves interfere with the movement of this protein, and information can not be obtained in the next cell. In this study, articles published in the PubMed databases, the Congress Library of the Web and the Web of Science were reviewed. Articles related to the study's purpose were selected. The present article is the result of a study and a summary of relevant articles available. Memory is a network. Any disruption occurring in an area of the network will interfere with the entire network. Elect $r$ omagnetic waves affect the brain and memory system and cause impairment in their performance. The brain consumes between $5 \%$ and $10 \%$ of total oxygen, so mobile phone waves reduce oxygen uptake by hemoglobin and lower oxygen levels reach the brain.Conclusion: Environmental information, magnetic waves, sound wave, heat, cold and what is affecting the touch, become electro magnetic waves entering the first receiver. The first effect of waves is the disruption of normal brain funct ion. Research results show that any waveguide with an $y$ frequency that can penetrate the brain cells may interfere with memory, learning and memorization. Studies have shown that mobile phone waves decrease oxygen uptake of hemoglobin. Being away is a mobile phone, especially when you sleep.

\section{P264}

Provides a Method for Detecting Left and Right
Hand Movement Imaginations in the Brain by Using Electroencephalogram Signal and Linear Propagation Coefficients (LPC) for Mel-Frequency Cepstral

\section{Seyed Amirhosein Mousavi, Maryam Naghavizadeh}

Department of Biomedical Engineering, Faculty of Engineering, Mashhad Branch, Islamic Azad university, Mashhad, Iran

The Neuroscience Journal of Shefaye Khatam, 2019; 7(3): P264

Introduction: The human brain is a very vital member of the body and controls the behavior and movement of the body. In this study, a method for classifying electron-phonogram signals based on neural network is presented. The first stage of the electroencephalogram signal is recorded. For this purpose, the BCI competition II database is used and the data set number 3 is used. In the second stage (7-13), signals are pre-processed and filtered appropriately. In the third stage, the alpha and beta band signal $(13$ to $30 \mathrm{~Hz})$ are extracted. In the fourth stage, the signal of the linear predictor coefficients of the Mel-frequency cepstral is derived. In the fifth step, the classification is performed by using a Support vector machine (SVM) classifier and the K nearest neighbors algorithm (K-NN). Conclusion: The best results from splitting Movement imagery for a support vector machine with a sensitivity of $92.88 \%$ are obtained.

P265

\section{Autism and Food Allergy}

\section{Mohammad Reza Khakzad', Elahe Karazhian'}

${ }^{1}$ Innovative Medical Research Center, Department of Immunology, Faculty of Medicine, Islamic Azad University, Mashhad, Iran ${ }^{2}$ Innovative Medical Research Center, Department of Immunology, Islamic Azad University, Mashhad, Iran

The Neuroscience Journal of Shefaye Khatam, 2019; 7(3): P265

Introduction: The prevalence of autism and allergy has risen dramatically in the last two decades causing a major public health issues in the developed countries. One of the main issues discussed in this research is that, why despite a few numbers of foods are involved in food allergy, it's prevalence is rising worldwide? Neuroimmune system has an essential role in neuroinflammation and behavioral changes in autism. Today, we know that food allergies affect on neural responses. Mast cells are abundant in the deep parts of the central nervous system. Mast cells have numerous granules that during degranulation, release histamine and other mediators which can stimulate the central nervous system. Histamine $\mathrm{H} 3$ receptors predominantly exist in the brain. Mast cell interactions with the nerve cells result in neuroinflammation and neurodegeneration which can disrupt the blood-brain barrier (BBB). In addition, food intolerance, food chemical sensitivities, 
and food poisoning are non-immunological responses to food that have similar symptoms and signs of food allergy. Furthermore, some of the peptide fragments such as gluten and casein can mimic some chemicals in the brain called endorphins. The common clinical symptoms of patients with food allergies, other behavioral symptoms including sleep, social interaction and, learning disorders, irritability, stress, anxiety, and depression may be observed in the children suffering from Child food allergy. Conclusion: The key question is that do autistic children with food allergy, talk to their parents or teachers about their problems? Even in normal children with food allergies, many of these symptoms are hidden. This research focuses on the relationship between autism and child food allergies.

\section{P266}

Medical Treatment of
Inflammatory Drugs on Alzheimer's Disease:
Systematic Review and Meta-Analysis

\section{Zeinab Sadat Hosseini, Aghile Heidari, Neda Rabiei}

Student Research Committee, Mashhad Branch, Islamic Azad University, Mashhad, Iran

The Neuroscience Journal of Shefaye Khatam, 2019; 7(3): P266

Introduction: Alzheimer's disease is one of the neurodegenerative diseases which affected 50 million people in the world. Laboratory findings indicates that nonsteroidal anti-inflammatory drugs (NSAIDs) may improve the Alzheimer's disease. The aim of this study is to systematically reviewed available researches about the relation of NSAIDs and Alzheimer's disease. A systematic search was done using Embase, PubMed, Google Scholar and Science Direct for publications from 2013 to 2018. All retrospective, or prospective observational studies and cross-sectional studies, of Alzheimer's disease in relation to NSAID exposure were included. Data extraction was done using a standardized data extraction tool in Microsoft Office Excel 2013. All the analyses were done using Stata V.15.0. . Of 28 articles, 9 ones had the qualitative criteria for inclusion in our study. According to the 2 crosssectional and 3 case-control studies, the combined risk evaluate for progress of Alzheimer's disease was 0.36 $(95 \% \mathrm{CI}=0.34-0.58)$ for exposure with NSAID. In the observational investigations, the approximation was 0.68 (95\% CI $=0.59-0.79)$ for the 4 studies about the relation between lifetime using NSAID. Conclusion: According to the analysis, NSAIDs had a role in the protection against the progress of Alzheimer's disease and it may decrease the risk of Alzheimer's disease.

\section{P267}

Study the Affection of Methamphetamine on Neuroinflammation: Systematic Review

Mahtab Aghaee Jaghargh ${ }^{1}$, Mohammadreza Khojaste ${ }^{2}$
${ }^{1}$ Student Research Committee, Mashhad Branch, Islamic Azad University, Mashhad, Iran

${ }^{2}$ Mashhad Branch, Islamic Azad University, Mashhad, Iran

The Neuroscience Journal of Shefaye Khatam, 2019; 7(3): P267

Introduction: Methamphetamine(METH) is one of the most dangerous drugs worldwide. It can cause neurotoxicity, high irritability and other disorders such as depression, hypertension and schizophrenia. The aim of this systematic review is to investigate about METH effects on neuroinflammation in neurological disease. We searched on Pubmed, Iranmedex, SID and Google Scholar database from 2000 to April 2019 and found 219 articles that 190 of which were excluded from the research by investigating the titles and 16 others by checking abstract; finally 13 articles are included in our study. Based on the studies, the effect of METH on neuroinflammation, which was dose-dependent, was through the effect on gene expression (e.g. NF-KB) and immune factors such as cytokines (e.g., interleukin1 ( $\beta$, 6,10 and tumor necrosis factor $\alpha(\mathrm{TNF}-\alpha)$ ), chemokines and the receptors (e.g. Dopaminergic Receptor, TollLike Receptor and Caspase-11). In people with HIV infection METH increased HIV co-receptors. METH had a direct effect on Blood Brian Barrier (BBB), causing it to open and disrupt its function .Ras homolog enriched in striatum (Rhes) protein had an important role in METH-induced neuroinflammation. The effect of METH on dopaminergic neurons, that causes Parkinson's disease, was indirect - with Damaged neurons release danger-associated molecular patterns (DAMPs) such as high mobility group box-1 (HMGB1) - in central nervous system (CNS). METH derivatives, such as Methylenedioxymethamphetamine (MDMA) and Methiopropamine, also had inflammatory effects. MDMA causes neuroinflammation through the effect on microglial (CD11b), astroglial (GFAP) and dopamine neuron. Chemicals such as $\mathrm{H}_{2} \mathrm{~S}$ and asiatic acid had an anti-inflammatory effect on inflammation caused by METH. Conclusions: METH, bypassing $\mathrm{BBB}$ and affecting different cells in CNS leads to the inflammatory cytokinins secretions and finally causes neuroinflammation. Inhibiting the effect of METH on neural cells can prevent the prevalence of its hazardous effects.

\section{P268}

Potential Theraupatic Application of CRISPR Gene Editing System in Dravet Syndrome

\section{Faeze Khaghani, Majid Mojarrad}

Department of Medical Genetics, Faculty of Medicine, Mashhad University of Medical Sciences, Mashhad, Iran

The Neuroscience Journal of Shefaye Khatam, 2019; 7(3): P268

Introduction: Dravet syndrome (DS), is a childhood- 
onset severe form of epilepsy which usually is a result of de novo mutations in SNC1A gene that encodes alpha1 Subunit of NaV1.1 (Voltage-gated Na+ channel). Valproic acid as the first line treatment for Dravet syndrome patients only controls seizure which means there is no cure for it up to now. CRISPR as a gene editing tool seems to be an appropriate potential therapeutic strategy for Dravet syndrome. Different CRISPR gene editing systems like base editing and HDR according to type of mutation can be done. Base editing is a potential appropriate strategy for point mutations in which a or $\mathrm{T}$ should be changed to $\mathrm{G}$ or $\mathrm{C}$ or vice versa. HDR is the other system which works via the cells homologous recombination dependent repair system. Delivery of CRISPR component to CNS can be done using AAV vectors which were shown to infect neurons quite efficiently. these vehicles can be administered to patient either systemically, intrathecally or intracranially. This procedure as a personalized medicine can be optimized and fit for every individual needs and also avoid over treatment and under treatment because some patient still is resistant to the existing treatments. In addition to being specific to patients it will be a certain cure and have long lasting effects in contrast to current treatments. Conclusion: Choosing and discovering the best delivery system like AAV based vectors will make this strategy a safe and cost-effective and in brief a typical potential theraupatic strategy for Dravet syndrome.

\section{P269}

\section{Neuropharmacological Effects of Ocimum Basilicum}

Atena Akbari, Farimah Beheshti ${ }^{2}$, 3, Masoumeh Gholami $^{2}, 3$, Mahmoud Hosseini', Hassan Azhdari Zarmehri ${ }^{2}$

'Student Research Committee, Torbat Heydariyeh University of Medical Sciences, Torbat Heydariyeh, Iran

${ }^{2}$ Neuroscience Research Center, Department of Medical Basic Sciences, Torbat Heydariyeh University of Medical Sciences, Torbat Heydariyeh, Iran

${ }^{3}$ Department of Neurocognitive Sciences, Psychiatry and Behavioral Sciences Research Center, Mashhad University of Medical Sciences, Mashhad, Iran

The Neuroscience Journal of Shefaye Khatam, 2019; 7(3): P269

Introduction: Ocimum basilicum (OB), known as sweet basil, is a popular Chinese medicinal herb of the family Labiatae. It is a perennial plant with yellow, white, and violet flowers, and oval leaves which have a sharp tip. It has been reported to contain a number of interesting compounds, such as mono-terpenoids, sesquiterpenoid, triterpenoid (ursolic acid) and flavonoid. Ocimum basilicum and other species of the same genus are used as medicines for the treatment of central nervous system (CNS) diseases and the essential oil of Ocimum basilicum. (OB) was screened for some CNS activities and used to treat headaches, diarrhea, helminthes infestation, inflammation, and pain. We searched the literature available in Scopus, PubMed and Science Direct databases for English articles published until 1990. For this purpose, we used 5 keywords for R. damascena, 3 for animal models and 7 for nervous system disorders. In this article we summarized neurofarmacological effects of Ocimum basilicum. There are not many studies have been done on the effects of this plant on the nervous system but these studies shown that ocimum basilicum used for the treatment of anxiety, nerve pain, convulsions and a variety of neurodegenerative disorders. In addition, we believed ocimum basilicum have been several effects on the central nervous system, such as anti-seizure, analgesic and antiinflammatory effects. The present review aimed to shown neurofarmacological effects of the plant on nervous system. Due to its inhibitory effects on the production of inflammatory mediators such as prostaglandins it can use to treatment nervous system disorders.

\section{P270}

A Review of the Noroprotective Effect of Medicinal Plants Against the Inflammation in The Treatment Cerebral Ischemia

\section{Seyedeh Mahdieh Khoshnazar', Siavash Parvardeh', Mohammad Reza Bigdeli ${ }^{1}$}

${ }^{1}$ Faculty of Life Sciences and Biotechnology Shahid Beheshti University, Tehran, Iran

${ }^{2}$ Department of Pharmacology, Faculty of Medicine, Shahid Beheshti University of Medical Sciences, Tehran, Iran

The Neuroscience Journal of Shefaye Khatam, 2019; 7(3): P270

Introduction: Stroke as third largest the reason for long term disability and death after cancer and cardiovascular disease and the second largest cause of pat physiological damage in adult. Approximately $60 \%$ of the population who have had cerebral ischemia with some form of disability such as paralysis, loss of sensory and motor function, loss of memory, language problems, and vision problems. The use of herbs and medicinal plants in different countries is increasing. Considering the intricate pathological process of cerebral ischemia, the use of one single agent does not seem ideal for treatment. Hence, studies in search of impressive compounds and methods are underway. Today, herbal medicine is used as alternative or complementary therapies with fewer side effects. New findings propose that some herbal medicine have the ability to aim multiple pathophysiological processes involved in cerebral ischemia including oxidative stress, inflammation, and apoptotic cell death. This study aimed to introduce the pathogenesis of cerebral ischemia and review the effects of medicinal plants and their mechanisms of action in cerebral ischemia. In this study is an overview survey about herbal medicines that evaluated in different resources and scientific databases including PubMed, ISI, Scopus, the 
title of herbal medicines and their keywords in treating cerebral ischemia. The review of administers studies showed that medicinal plants and their compounds are capable of diminishing infarct volume, cerebral edema, blood-brain barrier, deficit neurology through reducing oxidative stress also reducing the expression of inflammatory cytokines, microglial and astrocyte activities. Moreover, these plants are able to inhibit apoptotic protein expression and elevate anti-apoptotic protein expression. Conclusion: Therefore, Medicinal herbs and their compounds can be used as new sources against cerebral ischemia.

\section{P271}

The Effect of Nervous System Inflammation in Alzheimer Progression

\section{Neda Mohammadi}

Department of Neuroscience, Faculty of Advanced Medical Sciences and Technologies, Shiraz University of Medical Sciences, Shiraz, Iran

The Neuroscience Journal of Shefaye Khatam, 2019; 7(3): P271

Introduction: Alzheimer disease (AD) as a progressive neurodegenerative disorder is the most common cause of dementia in the elderly which affect about $9.5 \%$ of people above 70 years old all over the world. AD commonly begins with memory deficit and cognitive functions (executive functions, motor learning, attention, planning, reasoning ...) impairment and eventually leads to death. Regarding $\mathrm{AD}$ adverse and negative effects on the quality of life, life expectancy, and societal costs, attention to factors which are involved in its cause and progression is important. Multiple factors, including age, sex, and genetics are involved in AD disease. Among these factors inflammation is recognized as a major cause. Even though the inflammation process is necessary for tissue homeostasis and proper function but super inflammatory response can be a source of additional damage to cells and is related to neuronal death in AD. Damaged and dysregulated neurons interactions, activate microglia, produce and secrete inflammatory factors, including eicosanoids, cytokines, chemokines, reactive free radicals and proteases and create inflammation which result in AD chronic progression. So inflammation in $\mathrm{AD}$ patients nervous system is devastating. In conclusion, evidence shows harmful effects of neuroinflammation in AD neurodegenerative disease and people who use anti-inflammatory drugs are less likely to develop AD disease and chronic usage of these group of drugs can diminish the risk of AD and might be an effective form of treatment which could suspend or prevent the occurrence of AD disease. Conclusions: This paper reviews the effect of nervous system inflammation and anti-inflammatory drugs in $\mathrm{AD}$ progression.
P272

\section{A Review on Genetic Mutant Mouse Models in Epilepsy Studies}

\section{Elham Aminifar, Pouya Ghaderi}

Student Research Committee, School of Medicine, Mashhad Branch, Islamic Azad University, Mashhad, Iran

The Neuroscience Journal of Shefaye Khatam, 2019; 7(3): P272

Introduction: Animal models that are used in epilepsy studies must be able to show spike and wave discharge (SWDs) activity in the cortex and thalamus as the land mark of epilepsy in human form. There are several epileptic mutants mouse models such as tottering, lethargic, stargazer, mocha, slow-wave-epilepsy and ducky. Tottering mice are the best model for ataxia. The manifestation of "tottering" seizures is same as human. The frequency of SWDs in these mice is higher (6-7 Hz) than human. The phenotype of "lethargic" mouse appears after 15 days of age by ataxia and lethargic behavior accompanied to focal motor seizures. Generalized cortical SWDs are the second seizure type which is recorded in their EEG. The characteristic of their seizures is the same as human and tottering seizures. The phenotype of "stargazer" is similar to the other mutant mice including ataxia, paroxysmal dyskinesia and head tossing. The characteristic of seizures in "mocha" is same as stargazer mouse. The frequency of SWDs is 6-7 Hz and phenotype accompanied to ataxia, tonic-clonic seizures, pigment dilution, and increased bleeding time. SWDs in "slow-wave-epilepsy" mouse appear with frequency of $3-4.5 \mathrm{~Hz}$ during seizures. It has been considered as a valid model for epilepsy due to seizure phenotype and anti-epileptic-drug responses. This exchanger regulates the hemostasis, cell volume and mitogenic responses to growth factors. Ataxia, tonicclonic seizures and cerebellum degeneration are some phenotype appeared in these mutants beside of absence seizures. The frequency of SWDs in "ducky mice" same as the most of mice model of epilepsy is $6 \mathrm{~Hz}$. Ataxia, limb dyskinesia, and developmental dysgenesis of some brain areas like cerebellum, medulla and spinal cord are some additional phenotypes that exist in these mice. Conclusion: In conclusion, epileptic mutants mouse are favored models in neurobiology and neuropharmacology for the study of abnormal brain synchronization, but they have pioneer role in the neurogenic analysis of seizure disorders and their number are restricted by the rate of mutations, feasible dysfunctions. Also, in the mutant mousses models generally a single gene underlie the epileptic phenotype (SWDs), however, in humans a polygenetic mode of inheritance occurs.

\section{P273}

The Application of Polymer Scaffolds and Stem Cells 
to Repair the Nervous System

Maryam Alipou ${ }^{1}$, Mohammadreza Bigdeli ${ }^{1}$, Hadi Aligholi $^{2}$, Bahramrasoulian ${ }^{3}$, Mojtaba Khaksarian ${ }^{3}$

${ }^{1}$ Department of Animal Sciences and Biotechnology, Faculty of Life Sciences and Biotechnology, Shahid Beheshti University, Tehran, Iran

${ }^{2}$ Department of Neuroscience, Faculty of Advanced Medical Sciences and Technologies, Shiraz University of Medical Sciences, Shiraz, Iran

${ }^{3}$ Razi Herbal Medicines Research Center, Department of Physiology, Lorestan University of Medical Sciences, Khorramabad, Iran

The Neuroscience Journal of Shefaye Khatam, 2019; 7(3): P273

Introduction: Today, the use of cell therapy and tissue engineering is used in the treatment of many neurodegenerative diseases of the central nervous system, like ALS. Common treatments for these diseases are the use of autologous tissue around the tissue, but due to the complications of these methods, including the prevalence of post-surgical infections, the use of these methods always has been limited. For this reason, the use of tissue engineering and polymer scaffolding in the field of nerve reconstruction has attracted much attention. Successful nerve tissue engineering is fundamentally dependent on cellular regulation and tissue development through the creation of a synthetic scaffold that is an analog of the extracellular matrix, which can support 3D cell cultures. Therefore, there is a need to create synthetic scaffolds that are biocompatible, biodegradable, disabling the immune response to biomaterials to support nerve growth. Chitosan and alginate, including polymers that are used in this field because of having the mentioned properties. Stem cells are a group of undifferentiated cells that are capable of differentiating into a variety of specialized cells. The discovery of these cells in the central nervous system has created new hopes for the treatment of degenerative diseases of the central nervous system, such as stroke, Parkinson's disease, Alzheimer's, and spinal cord injury. Conclusion: Therefore, the combination of polymer scaffolds and stem cells can be promising for the treatment of neurological diseases.

\section{P274}

Inhibition C1q Proteins: A Way to Control Seizures in Epilepsy Inhibition C1q Proteins

\section{Elahe Safari $^{1}$, Pouya Ghaderi ${ }^{2}$, Zeinab Boostani ${ }^{1}$}

${ }^{1}$ Student Research Committee, Faculty of Nursery and Midwifery, Mashhad Branch, Islamic Azad University, Mashhad, Iran

${ }^{2}$ Student Research Committee, Faculty of Medicine, Mashhad Branch, Islamic Azad University, Mashhad, Iran

The Neuroscience Journal of Shefaye Khatam, 2019; 7(3): P274

Introduction: Epilepsy is a kind of chronic neurological disorders characterized by seizures. About $1 \%$ of the world's population have epilepsy and almost $80 \%$ of cases occure in developing countries. The Clq protein, the initiation protein of classical complement cascade associated with the immune inflammatory response, has been shown to be important in synaptic pruning through actions involving microglia. $\mathrm{Clq}$ and other protein supplements concentrate in synaptic regions where synaptic structures become weak or lost due to the activity of phagocytosis by microglials. Studies on rats show significant increase in $\mathrm{clq}$ and its components $(\mathrm{C} 3$, ic $3 b$ ) in epileptic mouse compared and the most increase was observed in the level of ic $3 b$, which was significantly associated with the frequency of seizures. Conclusion: In conclusion, it seems that level of C1q protein increases in seizure disorders, thus we can control the inflammation by inhibition the protein and prevent the progression of disease, however it needs to do more researches on different animal models and human.

\section{P275}

Bioinformatics Prediction and Introducting of Some Targeting Mirrnas of EPM2A and NHLRC1 Genes in Lafora Progressive Myoclonus Epilepsy Patients and Draw Networks of Interactions Bethween miRNAs and Genes

\section{Zahra Heydarei, Masoumeh Massah ${ }^{2,3}$, Ali Abedi', Samira Rahimirad ${ }^{3,4}$, Mansoureh Azadeh ${ }^{1,3}$ \\ 'Department of Basic Sciences, Najafabad Branch, Islamic Azad University, Najafabad, Iran \\ ${ }^{2}$ Department of Biochemistry, Najafabad Branch, Islamic Azad University, Najafabad, Iran \\ ${ }^{3}$ Zist Fanavary Novin, Biotechnology Institute, Esfahan, Iran ${ }^{4}$ Parand Branch, Islamic Azad University, Parand Iran}

The Neuroscience Journal of Shefaye Khatam, 2019; 7(3): P275

Intrudaction: Epilepsy is a common neurological disorder with freguent seizures and decreased mental function. One of its types is Lafora disease (LD) is mutation in genes EPM2A and NHLRC1 that it leads to the accumulation of in soluble glycogen deposits in the neurons. Vrious ways have been derised to treat Epilepsy that one of them is the use of miRNAs in terfernce small. MiRNAs intracts translation and actually expressing Epilepsy genes with the effection the transcription (mRNA) of these genes. Methods: Sites NCBI, SID. ir, PubMed were used to obtain information about the genes involved in the disease. In this study to evaluat the single-nucleutide poiymorphism (SNP) in the genes and miRNA related with them were used miRBAS, MIRNASNP databases. Correlation between genes and miRNAs and also bioinformatics review by software miRWALK2, the intereonnection network between genes and microcon trollers was selected. Result: Research on the sites indicated that these EPM2A and NHLRC1 genes are most importand those are involve in Epilepsy. These 
genes, respectively, order the production of proteins called Lafora and Malin. These protein have been considered in a complex but incomplete pathway described in the memory of glycogen metabolism. Reviews is shown that the transcription of these genes in 3'UTR site consists of two SNP with high freguensy. In EPM2A rs11966789 AC with has-miR-3943 and in NHLRC1 rs11966789 C/G with has-miR-186-5P couble by high affinity. The signal patways of DAVID and Kegg site showed that miRNAs of this research have in terfering effects on some of the cancerous genes such as K-Ras, MDM2. Conclusion: The setaps grid is drown between the set of genes and miRNAs wich are potentially involved in epileptic seizures, can clavity the role of miRNAs in the development and progression of the disease.

\section{P276}

The Use of Ferrule Magnets Nanoparticles with A Polymeric PLA Polymer Coating for Temozolamide to Treat Brain Tumors

\section{Parvin Pourmousumi}

Department of Medical Engineering, University of Tehran, Tehran, Iran

The Neuroscience Journal of Shefaye Khatam, 2019; 7(3): P276

Introduction: Today, an attempt is made to treat brain tumor (glioblastoma), one of the most common primary malignant tumors of the central nervous system. Although therapeutic options, such as chemotherapy, have progressed greatly, but because of the presence of a blood-brain barrier that reduces the effectiveness of the drug It turns out. Now using nanotechnology and drug loading, it's easy to cross the blood-brain barrier and has a better target for cancer cells and more penetration into the tumor, leading to greater efficacy in the tumor for treatment. Temozolamide, an effective therapeutic agent for the treatment of brain tumors, was conducted in this study. Using the ferritic nanoparticles that was loaded with Temozolamide into the encapsulated PLA (poly lactic acid), the timosulamide drug delivery with ferric nanoparticles with poly lactic acid coating has been activated with an OX26 monoclonal antibody, and with Transmission receptor targeting tumor cells (glioblastoma) used for expression of the receptor. The temozolamide test specification of nanoparticles was examined and the physiological conditions were evaluated and the extent of penetration of the drug into cancer cells was investigated. From two Glioblastoma cell U215, U87 for evaluation of drug and laboratory toxicity It is used to indicate the anti-cancer activity of temozolamide and to work with monoclonal antibodies to fixed transfusion receptors, which is beneficial in increasing glioblastoma infiltration. Conclusion: The results of this study are a good basis for the treatment of glioblastoma tumors, which is very effective in diminishing the tumor with the use of nanosized particles of carriers of temosulamide, coated with polycarboxylic acid polymer, for targeting and penetration of the tumor. And he has a therapeutic role.

\section{P277}

The Effect of CD39 Expression on Multiple Sclerosis Recurrence: Systematic Review

Valareza Alizadeh ${ }^{1,2}$, Mohammadreza Khojasteh ${ }^{1,2}$, Mohammadreza Taheri, ${ }^{1,2}$, Parsa Shoqi, ${ }^{1,2}$

${ }^{1}$ Mashhad Branch, Islamic Azad University, Mashhad, Iran

${ }^{2}$ Student Research Committee, Mashhad Branch, Islamic Azad University, Mashhad, Iran

The Neuroscience Journal of Shefaye Khatam, 2019; 7(3): P277

Introduction: multiple sclerosis is an autoimmune disorder mainly affecting young adults and characterized by destruction of myelin in the central nervous system. This disorder can have a recurrence period. $\mathrm{Cd} 39$ is an acidic glycoprotein and ectonucleotidase found on the surface of activated lymphocytes of all classes, endothelium, and various accessory cells within tissues. Recurrence is the return of a sign, symptom, or disease after a remission. We searched PubMed, Google Scholar and SID search engine with acute key words "multiple sclerosis", "cd39 gene" and "recurrence". As a result of our research, 43 articles were found respectively. All articles were published between 2010 until 2019 and have ethical considerations. All the studies, use human samples. After investigating their titles 22 articles were excluded and by reading abstracts 15 articles excluded. Finally 6 articles was included. Two articles of these studies revealed that, there was a positive association between recurrence of multiple sclerosis and CD39expression. The result of on study showed the CD39 expression in RRMS was significantly decreased. But the other study shows that, the CD39 expression level in RRMS patients hasn't significant difference with other MS patients. Another article results indicates, mRNA level was $70 \%$ higher in RRMS patients than other MS patients $(\mathrm{p}=0.0002)$. But the last article results shows that CD39 expression can inactivate extracellular ATP (as a natural exhibitor multiple proinflammatory factor) and reduces the inflammation effects. Conclusion: Until now the number of studies was done in this field was low. We can get more reliable results, if more studies tend to this field. As a result we think CD39 expression increase in RRMS patients to decrease extracellular ATP and decreasing proinflammatory effects.

\section{P278}

A Review of Nutritional Recommendation by Traditional Persian Medicine for the Treatment of Alzheimer's Disease

Behjat Javadi ${ }^{1}$, Milad Iranshahi ${ }^{2}$ 
${ }^{1}$ Department of Traditional Pharmacy, Faculty of Pharmacy, Mashhad University of Medical Sciences, Mashhad, Iran ${ }^{2}$ Biotechnology Research Center, Pharmaceutical Technology Institute, Mashhad University of Medical Sciences, Mashhad, Iran

The Neuroscience Journal of Shefaye Khatam, 2019; 7(3): P278

Introduction: Alzheimer's disease (AD) is a neurodegenerative disease, which is the leading cause of dementia worldwide. Epidemiological studies emphasis on the crucial role of nutrition and diet in prevention and treatment of AD. In Traditional Persian Medicine (TPM), multiple dietary guidelines have been suggested to prevent and cure dementia. Therefore, we aim to survey TPM-recommended food-items for the treatment of dementia. In addition, epidemiological and pharmacological evidence supporting the effects of TPM-suggested foods in improving cognitive performance will be discussed. TPM anti-dementia food-items were retrieved from major TPM textbooks. Scientific databases such as Pubmed, Science direct, Google scholar and Scopus were searched using the TPM-reported foods to obtain pharmacological data supporting the anti-AD activities and relevant mechanisms of action. Pharmacological studies have been shown that most of the TPM-recommended dietary items can improve cognitive impairment and possess anti-amyloidogenic, etc. activities. Among them, Allium sativum, Crocus sativus, Melissa officinalis, raisin, Rosa damascna and Zingiber officinale have been found to possess stronger anti-AD activities. Phytochemicals including S-allyl-cysteine, diallyldisulfide, crocin, crocetin, rosmarinic acid, resveratrol, geraniol, 6-gingerol, 6-shogaol and cuminaldehyde also possess potent anti-AD properties. The above food items and phytochemicals exhibited antioxidant and AChE inhibitory activities and were capable of decreasing lipid peroxidation. They also have antiamyloidogenic effects, reduced cerebral plaques and $A \beta$-species, suppressed cerebral inflammation and inhibited $A \beta$-induced apoptosis through various mechanisms. Conclusions: Considering TPM anti$\mathrm{AD}$ dietary recommendations in future research can be beneficial for discovering and development of new medicines and nutraceuticals for the treatment of $\mathrm{AD}$.

P279

Effect of Cognitive Rehabilitation for Elderly with Mild Neurocognitive Disorder: A Review

\section{Zahra Khorrami', Javad Salehi Fadardi', Imanollah Bigdeli $^{2}$}

${ }^{1}$ Ferdowsi University of Mashhad, Mashhad, Iran

${ }^{2}$ Faculty of Educational Sciences and Psychology, Ferdowsi University of Mashhad, Mashhad, Iran

The Neuroscience Journal of Shefaye Khatam, 2019; 7(3): P279
Introduction: Mild neurocognitive disorder or mild cognitive impairment (MCI) is stage between normal aging and Alzheimer. This period is prodromal stage for a variety of dementing neurodegenerative disorders, including Alzheimer's disease specially in elderlies. cognitive rehabilitation is a complex collection of technique and non-pharmacological intervention which target cognitive functions due to enhance them. In the present review, the researchers intended to analyze the effects of cognitive rehabilitation interventions in the older people with MCI, in form of a review. Data sources were the Web of Science, Pub Med, and Google Scholar. Studies markedly varied in terms of intervention approaches, duration of cognitive rehabilitation, sample of study, selected outcome measures, design of study. A few studies evaluated fallow up or the impact of cognitive rehabilitation on conversion rates from mild neurocognitive disorder to dementia or normal aging cognition. Conclusion: Most of the studies demonstrates that patients with MCI benefit from a cognitive rehabilitation program. comprehensive model, structured, individualized program can improve the results of these interventions.

\section{P280}

Tert-Butylhydroquinone Introduced as an AntiEpileptic Agent in Animal Model

\section{Masoud Hashemi-Arabi, Sajad Sahab Negah, Ali Gorji}

Department of Neuroscience, Faculty of Medicine, Mashhad University of Medical Sciences, Mashhad, Iran

The Neuroscience Journal of Shefaye Khatam, 2019; 7(3): P280

Introduction: Epilepsy is a multifactorial and heterogeneous disorder. Molecular and biochemical events involved in its occurrence which is not fully understood and require more research. Oxidative stress is developing as a mechanism that may play a key role in the etiology of seizure induced neuronal death. In a specific pathologic process like oxidative stress generation the free radical called reactive oxygen species (ROS) result in oxidative damage to cellular proteins, lipids, and DNA. Episodes of seizures can be prevented by reducing causative or precipitating factors. Use of antioxidants can be effective in this way. Conclusion: We suggested that tert-Butylhydroquinone (tBHQ) as a phenolic antioxidant improved electrophysiological parameters and behavioral changes in PTZ induction seizure in rat.

\section{P281}

Immunomodulatory Effects of Stem Cell Therapy in Multiple Sclerosis

Sayyedeh Helya Mir Nourbakhsh ${ }^{1,2}$, Niloufar Jafari', 


\section{2, Seyed Isaac Hashemy}

${ }^{1}$ Student Research Committee, Faculty of Medicine, Mashhad University of Medical Sciences, Mashhad, Iran

${ }^{2}$ Department of Neuroscience, Faculty of Medicine, Mashhad University of Medical Sciences, Mashhad, Iran

${ }^{3}$ Department of Clinical Biochemistry, Faculty of Medicine, Mashhad University of Medical Sciences, Mashhad, Iran

The Neuroscience Journal of Shefaye Khatam, 2019; 7(3): P281

Multiple sclerosis (MS) is increasingly recognized as a serious, worldwide public health concern. Statics indicate that globally 2.5 million people suffer from MS. It's a chronic inflammatory condition of the central nervous system which is followed by autoreactive lymphocytes. The first serious discussions of MS treatment emerged during the 1990s with disease-modifying therapy (DMT); however, there wasn't enough evidence of improvement in patients treated this way. In the recent decade, immune reconstitution therapies have been identified, one type of which is autologous hematopoietic stem cell transplantation (AHSCT). AHSCT therapy is an immunoablative therapy and induces immune reconstitution by hematopoietic stem cells. The way to treat autoimmunity, what exactly happens in MS, is that immunoablative therapy suppresses autoreactive immune cells and allows stem cells to produce a new, self-tolerant, cluster. In the last 15 years, AHSCT has been demonstrated as an effective approach. Therapeutic trials indicated that transplantation can lead to disease remission, relapse prevention and recovery enhancement from paresis. AHSCT restores the regulatory T-cell network, reduces pro-inflammatory IL-17 response, normalizes microRNA expression and gene expression profiles. Despite compelling evidence that supports this treatment, the exact mechanism of its efficacy remains unclear. Little is known about how AHSCT limits or controls autoreactive lymphocytes. This indicates a necessity to further studies to address some biomarkers of clinical response to AHSCT in autoimmunity in order to clarify its function. Moreover, the disease reactivates in some patients due to reasons not yet understood. For this reason, also, further researches are essential to improve or sustain the therapeutic efficacy of AHSCT in autoimmunity.

\section{P282}

$\begin{array}{llccr}\text { How Personalized } & \text { Medicine } & \text { Would } & \text { Help } \\ \text { Amyotrophic } & \text { Lateral } & \text { Sclerosis } & \text { Diagnosis } & \text { and } \\ \text { Therapeutic } & \text { Intervention? } & & \end{array}$

Niloufar Jafari ${ }^{1},{ }^{2 *}$, Negar Khoshghamat ${ }^{1}{ }^{2}$, Seyyedeh Helya Mir Nourbakhsh ${ }^{1},{ }^{2}$, Seyed Isaac Hashemy ${ }^{3}$

'Student Research Committee, Faculty of Medicine, Mashhad University of Medical Sciences, Mashhad, Iran

${ }^{2}$ Department of Neurosciences, Faculty of Medicine, Mashhad University of Medical Sciences, Mashhad, Iran
${ }^{3}$ Department of Clinical Biochemistry, Faculty of Medicine, Mashhad University of Medical Sciences, Mashhad, Iran

The Neuroscience Journal of Shefaye Khatam, 2019; 7(3): P282

Amyotrophic lateral sclerosis (ALS), also known as Lou Gehrig's disease, is the most common fatal neurological disorder which leads to upper and lower motor neuron impairments via neuro-inflammatory and oxidative mechanisms. It has the highest incidence in individuals over 55 years old. Muscle atrophy and spinal cord sclerosis are some of the neuropathological features. No definite therapeutic intervention is known for ALS. Drug therapy is of the classic treatments. Although the outcome of different drugs application on animal models were promising, clinical assessments were not approval. Personalized medicine (PM) is about to defines specific and quantifiable biomarkers for ALS in order to facilitate its early diagnosis and help the clinicians to choose the best therapeutic intervention. Scientists have estimated that there are some stage-specific biomarkers of ALS , but, unfortunately, we still don't have enough proven and valid biomarkers. In 2017, for the first time, a study revealed specific MicroRNA (miRNA) variation like miRNA-20, miRNA-143-3p and miRNA-374b-5p in patients . It was suggested that these serum miRNAs may result from muscle denervation. Scientists suggest that MiRNA serum level may change years before disease onset and miRNA expression persists during disease progression. The implication of this is the possibility that miRNAs could be appropriate longitudinal biomarkers to facilitate prognosis, diagnosis and intervention of the disease. In addition, it is newly found that some RNA targeted therapies had noticeable outcome for neurodegenerative disorders like Parkinson disease. Hence, it could conceivably be hypothesized that by detecting specific biomarkers and applying that RNA targeted therapies, we would be able to stop disease progression years before the onset of its symptoms. Building upon the above statements, further studies are needed to reveal more specific and trustable biomarkers.

\section{P283}

Prospects for the Application of Mesenchymal Stem Cells in Alzheimer's Disease Treatment

\section{Sanam Dolati, ${ }^{1,2}$}

${ }^{1}$ Aging Research Institute, Tabriz University of Medical Sciences Tabriz, Iran

${ }^{2}$ Department of Immunology, Faculty of Medicine, Tabriz University of Medical Sciences, Tabriz, Iran

The Neuroscience Journal of Shefaye Khatam, 2019; 7(3): P283

Alzheimer's disease (AD) as a dementia and neurodegenerative disease, is mostly prevalent among people more than 65 years. AD is mostly manifested in the form of degraded mental function, such as losing 
memory and impaired cognitive function. Due to inefficiency of traditional pharmacological therapeutic approaches with no long-term cure, cell therapy can be considered as a capable approach in AD management. Therapies based on mesenchymal stem cells (MSCs) have provided hopeful results in experimental models regarding several disorders. MSCs enhance the levels of functional recoveries in pathologic experimental models of central nervous system (CNS) and are being investigated in clinical trials in neurological disorders. However, there is limited knowledge on the protective capabilities of MSCs in AD management. Almost, several experiments have suggested positive effects of MSCs and helped to better understand of AD-related dementia mechanism. MSCs have the potential to be used in $\mathrm{AD}$ the treatment through amyloid- $\beta$ peptide (AB), Tau protein and cholinergic system. This review aimed to clarify the promising perspective of MSCs in the context of $\mathrm{AD}$.

\section{P284}

Therapeutic Targeting of Autophagy in Alzheimer's Disease

\section{Mohamadreza Diba', Seyyede Nafise Vakili², Zahra Meshkat}

${ }^{1}$ Department of Biochemistry, Faculty of Medicine, Shiraz University, Shiraz, Iran

${ }^{2}$ Department of Virology, Faculty of Medicine, Shiraz University, Shiraz, Iran

${ }^{3}$ Department of Microbiology and Virology, Faculty of Medicine, Mashhad University, Mashhad, Iran

The Neuroscience Journal of Shefaye Khatam, 2019; 7(3): P284

As terminally differentiated nervous system cells, neurons may be specialized to fight viral infections without undergoing cellular selfdestruction. Autophagy, the cellular lysosomal degradation pathway, is emerging as one such mechanisms of neuronal antiviral defense. Autophagy has diverse physiological functions in cells, such as cellular adaptation to stress, routine organelle and protein turnover, and innate immunity against intracellular pathogens, including viruses. Much evidence for an antiviral role of autophagy is related to viruses that specifically target neurons, includingtheprototypealphavirus, Sindbis virus and the alpha-herpesvirus, herpes simplex virus type 1 (HSV-1). Autophagy acts as both an anti-viral and pro-viral pathway in the nervous cells, and the roles of autophagy depend on the virus, the cell type and the cellular environment. Autophagy is the very important mechanism in preventing neurodegeneration, and induced autophagy is essential for promoting cellular survival, viral antagonism of autophagy in neurons may lead to neuronal dysfunction and/or neuronal cell death in patients. Autophagic control of inflammation is one area where this pathway may have similar benefits for both infectious and neurodegenerative diseases beyond direct removal of the pathogenic agents. Alzheimer's disease $(A D)$ is a neurodegenerative disorder that characterized by the formation of senile plaques and neurofibrillary tangles (NFTs) in the hippocampus and cortex. Many studies in recent years were showed significant associations between $\mathrm{AD}$ and various pathogens, specially Herpes simplex virus type 1(HSV-1). This review provides background information on the roles of autophagy in immunity and neuroprotection, and then discusses about the therapeutic targeting of autophagy in Alzheimer's disease.

\section{P285}

The Balance of Th17 Versus Treg Cells in Multiple Sclerosis

Aylar Erfanian Taghvaee ${ }^{1}$, Zahra Azimzadeh Tabrizi', Haleh Hosseini', Hamed Mohammadi ${ }^{2,3}$

${ }^{1}$ Student Research Committee, Alborz University of Medical Sciences, Karaj, Iran

${ }^{2}$ Student Research Committee, Tabriz University of Medical Sciences, Tabriz, Iran

${ }^{3}$ Department of Immunology, Faculty of Medicine, Tabriz University of Medical Sciences, Tabriz, Iran

The Neuroscience Journal of Shefaye Khatam, 2019; 7(3): P285

Among the wide range of the central nervous system (CNS)-related diseases multiple sclerosis (MS) has drawn much attention these days. MS is considered as a chronic autoinflammatory disorder, which leads to brain inflammation and may involve a break tolerance. Experimental autoimmune encephalomyelitis (EAE) is an animal model of MS. MS is characterized based on Antigen-reactive T cells with distinct cytokine secretion resulting in attacking myelin sheaths around axons in the brain and spinal cord. Although previously it was considered that Th1 cell subset drives MS, today it is demonstrated that pathogenic Th17 cells and the impaired balance of Th17/Treg axis are implicated in the pathogenesis of MS. Th17 key cytokines play an important role in MS. As an example, IL-17, the hallmark cytokine of Th17, induces neutrophils infiltrations and ILs productions. It also shows a positive correlation with MS severity. IL-6 and tumor growth factor $\beta$ (TGF- $\beta$ ), two main cytokines in Th17 differentiation affect neurogenesis like glial cells. IL-1 $\beta$ is also involved in this process via disrupting blood-brain barrier, enhancing microglia and astrocytes activity resulting in accelerating nerve cell demyelination. Regulatory $\mathrm{T}$ cells (Treg) are also implicated in the pathogenesis of MS. They can decrease MS progression, and depletion of Tregs exacerbates EAE. Also, IL-10, secreted by 
Tregs attenuates EAE severity. Overall, Th17 and Treg special cytokines and factors leading to an imbalance of Th17/Treg axis are considered to be essential in MS and EAE immunopathogenesis. In this review, we covered various factors, which can cause an imbalance between Th17 and Treg subsets in MS and EAE.

\section{P286}

\section{The Effects of Herbal Medicine on Epilepsy}

\section{Mahdi Isazadeh, Mohammad Hassan Dehghani}

Student Research Committee, Faculty of Medicine, Aja University of Medical Sciences Tehran, Iran

The Neuroscience Journal of Shefaye Khatam, 2019; 7(3): P286

Epilepsy is the fourth most common neurological disorder and affects people of all ages. In past centuries before modern seizure medicines were developed, people with epilepsy and their doctors looked for a remedy in various herbs and combinations of herbs and now People with epilepsy and their doctors are expressing growing interest in alternative therapies too. Although antiepileptic drugs (AEDs) help most people control their symptoms, these do not work for everyone. Furthermore, some people are concerned about the longterm safety of these drugs. Traditional herbal therapy plays an important role in the treatment of epilepsy. Though herbal therapy is mostly used in antiepileptic treatment, there is a lack of robust evidence for efficacy and toxicity of most herbs. Besides, the herbal medicine should be subject to evidence-based research. In this review article, our purpose was to introduce the effects of herbal medicine on epilepsy. However, hundreds of herbal medicines have been investigated in the available studies. Some commonly used herbal medicines for epilepsy are Zizyphus jujube, Taxus wallichiana Zucc (Himalayan Yew), Ganoderma lucidum, Salvia miltiorrhiza (Danshen or Chinese red sage), Uncaria rhynchophylla and etc. The overwhelming majority of these article are based on animal experiments. The lack of clinical data places constraints on the clinical suggestion of herbal medicine. Many reports on the effectiveness of complementary treatments for epilepsy come from personal experience, and from studies that are not considered conclusive. Most importantly, people should always talk to their doctor before trying natural treatments to help ease their symptoms.

\section{P287}

Genetic Rat Models of Absence Epilepsy: A Review on Similarities and Differences with Absence Epilepsy in Human

\section{Aghile Heydari', Pouya Ghaderi ${ }^{2}$}

${ }^{1}$ Student Research Committee, Faculty of Nursery, Mashhad Branch, Islamic Azad University, Mashhad, Iran
${ }^{2}$ Student Research Committee, Faculty of Medicine, Mashhad Branch, Islamic Azad University, Mashhad, Iran

The Neuroscience Journal of Shefaye Khatam, 2019; 7(3): P287

Nowadays two model groups are reported for the spontaneous occurrence of spike and wave discharges (SWDs) for seizures in untreated rats include GAERS and WAG/Rij rats, which are independently derived by inbreeding of normal wistar rats. Different studies with different approaches revealed that these two rats have similar feature to human epilepsy. In GAERS, SWDs are first detected around 30 days of age. The most SWDs occur around 4 to 6 months. There are not any different in the characteristics of SWDs in the males and females, that represent genetic transmission is autosomal. The frequency of SWDs in these rats is $7-10 \mathrm{~Hz}$. The antiepileptic drugs are able to suppress the seizure attacks. The most important different of seizures in GEARS and human is the higher frequency of SWDs. It is believed that polygenetic mutation involve in seizures genesis in this rat model. The WAG/Rij strain is a subline of the WAG strain that was derived from wistar supply. SWDs are emerged at around 60 to 80 days in WAG/Rij. At 6 months of age all of the animals show SWDs and it occurs alternatively until the death of the animals. SWDs in the WAG/Rij rats are very age dependent. Generally burst activities last 1-30 $\mathrm{s}$ and spike-wave frequency is $7-11 \mathrm{~Hz}$ in the cortical EEG of adult WAG/Rij rats. Behavioral manifestation of seizures is same as GAERS rats. It is noticeable that beside of high frequency of SWDs, the age of SWDs initiation is different from human. The pharmacological responses to anti-epileptic drugs in these rats are same as human.In conclusion, although GAERS and the WAG/Rij strains of wistar rats have limitations, but they have asserted to be valid and predictive models of human epilepsy.

\section{P288}

Neurophysiology and Role of Inflammation in Pain Abolfazl Barzegar Bafrouei', Moosa Javdani²

${ }^{1}$ Faculty of Veterinary Medicine, Shahrekord University, Shahrekord, Iran

${ }^{2}$ Department of Clinical Sciences, Faculty of Veterinary Medicine, Shahrekord University, Shahrekord, Iran

The Neuroscience Journal of Shefaye Khatam, 2019; 7(3): P288

Nociceptive stimulates are transpired by the duration of the stimulus and the intensity of its application. This duration/ intensity combination is resolute in dividing "physiological pain" from "pathological pain". In pathological pain, the duration and/or intensity of the tissue attack are unnatural. Apart from the instantaneous clinical result of such stimulation, the occurrence of sensitization happens: sustains and amplifies threshold of the nociceptors involvement, and 
derivation of the signal in the spinal cord is facilitated (central sensitization), which excitability threshold of the nociceptors is pronouncedly decreased (peripheral sensitization). This circle is instated very quickly, from a few minutes to a few hours after the start of tissue injury. It amplifies and sustains the pain. Nociceptors have a high activation threshold: they are just activated by stimuli of high intensity. The chemical agents participated in the activation of nociceptors include algogenic agents (those which invite pain) such as $\mathrm{K}+$ and $\mathrm{H}+$ ions and inflammatory mediators such as serotonin, substance $\mathrm{P}$, histamine, prostaglandins (PGs). These substances are emancipated during inflammation of all sources (tumor, trauma) and have a synergistic effect: they produce lower the nociceptors activation threshold and sustained activation of them. PGs are released when tissue is injured. Their demonstration is highly stimulated at the focus of the inflammation. prostaglandinE2 (PGE2) sensitizes the nociceptive afferents to other mediators such as bradykinin and histamine, which are potent activators of polymodal nociceptors. Serotonin increases the happening through its 5-HT3 receptors. Over time this occurrence is amplified further, as repeated stimulation invites the response to expand incrementally. Glutamate is one of the main mediators, through the alpha- amino-3hydroxy-5-methyl-4-isoxazole propionic acid (AMPA) and N-methyl-D-aspartate (NMDA) receptors. The AMPA receptors seem to be involved in the transmission of stimulations of short duration. Activation of NMDA receptors requires excitation of greater duration and/or intensity.

\section{P289}

Reflexology; Therapeutic Way to Relive from Head ache

\section{Seyed Hossein Vaezzadeh ${ }^{1}$, Hadi Khodadadi ${ }^{2}$}

${ }^{1}$ Department of Medicine, Faculty of Medical Sciences, Azad University of Medical Sciences, Mashhad, Iran

${ }^{2}$ Department of Physiology, Faculty of Medicine, Mashhad University of Medical Sciences, Mashhad, Iran

The Neuroscience Journal of Shefaye Khatam, 2019; 7(3): P289

Headache is a type of referral pain that results from head deep-seated structures and is one of the most common complaints in neurology clinics. headache has two intra and extra cranial origin. Also, any type of traumatic, squash or fatal Stimulation of the meningeal blood vessels causes headache. Intracranial headaches Include: headache caused by meningitis, headache caused by low CSF pressure, migraine, alcoholic headache and tension-type headache. The lifetime prevalent headaches are migraine and tension-type headache but tension-type headache has more prevalence. These types of headache seem to be caused by an abnormal vascular phenomenon although its mechanism has not been known so far. Headache Treatment contains pharmacologic and non-pharmacologic interventions. One of the non-pharmacologic interventions which can reduce the frequency and severity of headache is reflexology. It could be done by two major ways; hand and foot reflexology which can alleviate pain and decrease the tension and anxiety in patient under any circumstances. Reflexology is based on the assumption that the peripheral nerves in feet, hands and ears are related with other parts of body. pressure on Specific reflex points can reduce symptoms in other parts of the body. For example, nerve involvement in the thumb can reduce headache. The way this method works as a painkiller in body is probable stimulation of serotonin secretion and vasodilation which can raise the pain threshold. in conclusion, using this method can alleviate pain in all kinds of headache and also it can be used by patient himself as a complementary alternative medicine.

\section{P290}

Pathophysiology of Migraine Headache Based on Persian Traditional Medicine

\section{Majid Anushirvani, Ali Gholampour}

Faculty of Persian Traditional Medicine, Mashhad, Iran

The Neuroscience Journal of Shefaye Khatam, 2019; 7(3): P290

Migraine is a type of primary recurrent vascular headache that are chronic and debilitating and characterized by pulsating in the temporal area, nausea, vomiting, photophobia and phonophobia, with moderate to severe pain, and duration of headache is from 4 to 72 houres. In the literatures of Persian medicine (P.M), 28 types of headache have been described such as shgaghigheh headache. This headache occurs periodically with throbbing pain in the right or/and left side. Some of the clinical features of "Shaghigheh" headache are highly compatible with migraine. According to the P.M. literature, it is believed that "Shaghigheh" most often is caused by substantial dystemperament that occurs inside the brain or internal/external membranes of the brain. Shaghigheh can generally categorize into hot and cold types. Hot shaghigheh headache is caused by hot wind and hot humors such as sanguine and bile, and cold one due to cold wind, phlegm and black bile. In the canon of medicine by Avicenna, he has divided the headache according to their bio-psychological etiology as follows: The efficient/subjective causes:

The qualitative internal/external causes of heat and cold that produce headaches such as exposure to sunlight, cold weather, eating hot and spicy foods. In these cases substances have no role in the pathogenesis of the disease.

Substantial causes: means biologic fluids and gases 
including humors, winds and vapors

The primary pathogenic site of the headache:

headache occurs primarily in different parts of the brain: Main headache (primary):

2) Headache arises from the participatory with other organs (secondary): a headache that occurs secondary to disorders in other organs (stomach, liver, uterus, spleen) or happens subsequently to a general bodily event (headache caused by fever).

\section{P291}

\section{The Speech and Language Defects in Parkinson Disorder}

\section{Fatemeh Fekar Gharamaleki', Sahar Bagherzadeh ${ }^{2}$, Faezeh Hemmati', Mina Ahmadi Kahjoogh ${ }^{3}$}

${ }^{1}$ Department of Speech Therapy, Faculty of Rehabilitation, Tabriz University of Medical Sciences, Tabriz, Iran

${ }^{2}$ Faculty of Rehabilitation, Tabriz University of Medical Sciences, Tabriz, Iran

${ }^{3}$ Department of Occupational Therapy, Faculty of Rehabilitation, Tabriz University of Medical Science, Tabriz, Iran

The Neuroscience Journal of Shefaye Khatam, 2019; 7(3): P291

Parkinson disease (PD) is a neurodegenerative disorder of central nervous system that causes partial or full loss in motor reflexes, speech, behavior, mental processing, and other vital functions. People with Parkinsonism suffer from speech impairments like dysphonia (defective use of the voice), hypophonia (reduced volume), monotone (reduced pitch range), and dysarthia (difficulty with articulation of sounds or syllables). Diagnosis and treatment of PD at early stage is important issue in speech and language pathology. We conducted a search in PMC, Web of Science, PubMed, Scopus, Medline, SID, Google Scholar and Ovid databases. The present information is available reviewing the articles from 1996 to 2019 through the search of the resources. speech and language problems occurs in about 90 percent of PD patients. The disorder impairs the expression and reception of language as well as reading and writing. Therefore, parkinsonian speech is characterized by respiratory and laryngeal problems such as lower fundamental frequency, monopitch and monoloudness, resonance imbalance, phonoasthenia and lack of articulatory integrity. They have dysphagia signs include delayed oral transit time, festinated tongue movement, uncontrolled bolus with premature loss to the pharynx, piecemeal deglutition, reduced movement of the hyolaryngeal complex, pharynx, tongue base, and epiglottis, esophageal motility and reflux issues. Parkinsonism assessments is generally based on four criteria of expression and comprehension, repetition and naming ability. Speech therapy is among one of the most important rehabilitation treatments for PD patients, which can help regain important cognitive and communication functions. An SLP will also teach to PD patients how to adapted and relearn speech and language, also can help PD patients regain cognitive function and memory, which can improve word retrieval and regaining problem-solving skills. Speech therapy methods can improve brain synaptic plasticity. The aim of therapy is to recover as much of speech as possible and/or find alternative ways of communicating.

\section{P292}

The Study of Cardiac Damage and the Risk of Leukemia in MS Patients Treated with Stem Cells

\section{Ali Motamed-Sanaye ${ }^{1}$, Sajjad Abbasi ${ }^{1}$, Maryam Moghimian', Masoumeh Fani}

${ }^{1}$ Student Research Committee, Gonabad University of Medical Sciences, Khorasan-Razavi, Iran

${ }^{2}$ Faculty of Medicine 'Gonabad University of Medical Sciences, Khorasan-Razavi, Iran

${ }^{3}$ Department of Anatomical Sciences, Faculty of Medicine 'Gonabad University of Medical Sciences, Khorasan-Razavi, Iran

The Neuroscience Journal of Shefaye Khatam, 2019; 7(3): P292

Multiple Sclerosis(MS) is a neurodegenerative disease that affects the brain and spinal cord. Cellular transplantation, from bone marrow or other sources, can be an effective treatment for people with certain types of cancer, such as leukemia and lymphoma. Cell transplantation is used to treat many myeloma and neuroblastoma and those with other types of cancer. The purpose of transplantation or bone marrow transplantation is to suppress the immune system with chemotherapy after the end of the radiation. After successful transplantation, bone marrow begins to form a new blood cells. Pubmed and Google Scholar search engines were searched for "Multiple Sclerosis", "Stem Cells", "Treatment" and "Leukemia", and the review articles of the last 5 years (2015-2019) were selected. Out of the total 490 articles found, 372 articles were only available in Abstract form and 118 articles were presented in full text from which 20 articles fully associated with the title were selected for the study. It is said that in addition to stopping the disease, a treatment through autologous hematopoietic stem cell transplantation (HSCT) heals injuries in the body. It is necessary to check the validity of this theory. To achieve this goal, it is necessary to carefully examine the effects of this therapeutic approach on important organs of the body such as the heart, the brain and on the probability of developing cancers such as leukemia. It is worth mentioning that immunosuppressive drugs are used for MS a powerful example of which is Mitoxantrone. According to some reports acute myeloid leukemia has been previously seen in people treated with Mitoxantrone for MS or cancer. The aim of this study is to check the risk of leukemia and heart disease in MS patients cured with HSCT. 
P293

Neurofilament Light Chain: A Possible Biomarker to Predict Development of HTLV-1-Associated Myelopathy/Tropical Spastic Paraparesis

Samaneh Abolbashari", 2, Zahra Meshkat ${ }^{1,3}$, Majid Ghayour-Mobarhan ${ }^{1,} 4$

${ }^{1}$ Department of Modern Sciences and Technologies, Faculty of Medicine, Mashhad University of Medical Sciences, Mashhad, Iran

${ }^{2}$ Student Research Committee, Mashhad University of Medical Sciences, Mashhad, Iran

${ }^{3}$ Antimicrobial Resistance Research Center, Mashhad University of Medical Sciences, Mashhad, Iran

${ }^{4}$ Metabolic Syndrome Research Center, Mashhad University of Medical Sciences, Mashhad, Iran

The Neuroscience Journal of Shefaye Khatam, 2019; 7(3): P293

Neurofilaments are the main components of the axonal cytoskeleton. Neuroaxonal damage which occurs in different chronic neurological disorders results in the release of Neurofilaments into the cerebrospinal fluid (CSF) and later on into the serum. Thus they are being introduced as potential biomarkers for the diagnosis and also the monitoring process of many acute and chronic neurologic diseases. Human T-Lymphotropic virus type 1 (HTLV-1) infection can lead to a progressive neurologic disease called HTLV-1-associated myelopathy/tropical spastic paraparesis (HAM/TSP). Patients suffer from myelopathic symptoms such as spastic paraparesis, lower limb sensory disturbance and bladder dysfunction. Diagnosis of HAM/TSP is mainly based on clinical finding and on the identification of anti-HTLV antibodies in serum and CSF. But it is sometimes difficult to differentiate between HAM/TSP and myelopathies of unknown origin. The use of potential biomarkers to differentiate these will therefore be quite beneficial. HTLV-1 bZIP factor (HBZ) which is a nuclear protein found in chronically infected cells with HTLV-1 is shown to be exclusively localized in the cytoplasm of peripheral blood mononuclear cells (PBMC) from patients suffering of HAM/TSP and thus is introduced as possible biomarker for HAM/TSP; but it is further needed to be studied. It has been shown that in HAM/TSP, only the motor and sensitive tracts of the spinal cord are damaged and the neurons are left intact; thus the degenerative process is limited to axons. This finding supports the possibility of Neurofilaments to be used as biomarkers in HAM/TSP. Also, another study has shown that levels of Neurofilament light chain (NFL) was significantly increased in Human Immunodeficiency virus (HIV) associated dementia. Since HTLV-1 and HIV are both retroviruses and have common pathological pathways; the utility of NFLs as biomarkers for the diagnosis of HAM/TSP should be considered in future studies.

\section{P294}

Chemical Models in Typical Absence Epilepsy Studies: A Review on Advantages and Disadvantages

Pouya Ghaderi, Vida Yegane

Student Research Committee, Faculty of Medicine, Mashhad Branch, Islamic Azad University, Mashhad, Iran

The Neuroscience Journal of Shefaye Khatam, 2019; 7(3): P294

There are several substances for induced absence seizure that used locally or systemically based on the aim of the study. Systemic Penicillin, low-dose pentylentetrazole (L-PTZ), the 4,5,6,7 tetrahydroisoxazolo $[4,5,-\mathrm{c}]$ pyridine-3-ol (THIP) model, and gamma-Hydroxybutyrate (GHB) model are the most common models for typical absence epilepsy as well as AY-9944 and methylazzoxymethanol acetate (MAM)-AY-9944 models administrate for induced atypical absence seizures. Local Administration of some antibiotics such as cephalosporin and penicillin onto the cortex or systemically at high doses can induce seizure in cats and rodents. Penicillin has limited effect in rodents rather than cats. Because of unstable penetration of penicillin through the bloodbrain barrier into the brain, produces multifocal spikes with only occasional bursts of bilaterally synchronous spike and wave discharges (SWDs) associated with a decrease in attention. In fact, seizures initiated through parenteral penicillin have minor similarity to clinical absence seizures in rats. PTZ is the most commonly used epileptic induced agent for systemic convulsive epilepsy. The extensive use of PTZ in different types of epilepsy is caused this model as an identifier of anticonvulsants and pro-convulsants. GHB is a GABA metabolite substance that produces naturally in the mammalian brain. After IP administration of GHB, electrographic and behavioral events occurs, similar to generalized absence seizures in the cats, rats, and monkeys. GBL a prodrug of GHB, increases the reproducibility and predictability of the GHB model of absence seizures. GBL is more common usage because of the consistency and rapidity of onset of its effect and has been shown to produce exactly the same EEG and behavioral effect as that of GHB. This model is very appropriate experimental model for the study of the mechanisms of bilaterally synchronous SWD production and screen for anti-absence activity of antiepileptic drugs in generalized absence seizures. THIP is a GABA agonist that induces bilaterally synchronous SWDs in rats. THIP is a selective GABA agonist for the extra synaptic Deltasubunits of GABAA receptors. This model is quantitated similar to GHB model.

\section{P295}

HTLV-1 and Central Nervous System Disease

Zahra Meshkat ${ }^{1}$, Mona Fani ${ }^{2}$ 
'Antimicrobial Resistance Research Center, Mashhad University of Medical Sciences, Mashhad, Iran

${ }^{2}$ Department of Virology, Faculty of Medicine, Ahvaz Jundishapur University of Medical Sciences, Ahvaz, Iran

The Neuroscience Journal of Shefaye Khatam, 2019; 7(3): P295

Introduction: Human T-lymphotropic virus type1 (HTLV-1) is a retrovirus that infects from 15 to 25 million people worldwide and is associated with tropical spastic paraparesis/ HTLV-1-associated myelopathy (TSP/HAM). TSP/HAM as a neurological disorder is associated with bladder and sphincter disturbances and minimal sensory loss. On the other hand, TSP/HAM involves faster women than men and is characterized by demyelinisation of the white and grey matter and meningo-myelitis of the lower thoracic cord. HTLV-1 can transmit to blood brain barrier (BBB) and enter into cells of the central nervous system (CNS). Consequently, this virus can infect lymphocytes produce pro-inflammatory cytokines, Interleukin 1 beta and interleukin 6 , that disrupts the BBB. In addition, infiltration of perivascular T-lymphocyte into CNS was reported. Actually, presence of $\mathrm{T}$ and macrophages cells in TSP/HAM spinal cord material and also, the up-regulation of HLA and cytokine molecules indicate that such an increase in immunologic activity in the CNS may be related to the pathogenesis of TSP/HAM. Conclusion: Generally, the most important factors to progress TSP/HAM are sex hormones and proviral load. IgG specific anti HTLV-I antibody in the serum and cerebrospinal fluid is one of others factor for detection of HTLV-1. The aim of current study is to present the complexities of HTLV-1 associated neurological disorders.

\section{P296}

\section{Painful Immunoglobulin-G4 Related Neuropathy}

\section{Reza Boostani, Marzie Taheri, Nahid Olfati}

Department of Neurology, Faculty of Medicine, Mashhad University of Medical Sciences, Mashhad, Iran

The Neuroscience Journal of Shefaye Khatam, 2019; 7(3): P296

Introduction: Chronic sensorimotor neuropathies are the dominant clinical presentation of a variety of disorders. Newly recognized entities need to be appreciated in evaluation of these disorders. A 38-year-old male patient presented to our department with one year history of progressive numbness and severe pain starting from lower limbs accompanied with a significant weight loss, constipation, urinary retention, and dry mouth during the last six months. On examination a bilateral proptosis without eye movement restriction, normal cranial nerve examination, decreased force and atrophy of the distal lower limb muscles were evident accompanied with abolished muscle stretch reflexes and decrease in pain and temperature sensation. Electrodiagnostic study was compatible with a chronic axonal sensorimotor neuropathic process. Laboratory evaluations revealed an increasing erythrocyte sedimentation rate up to 72 $\mathrm{mm}$ /hour and a positive c-reactive protein. Primary evaluations was negative for common vasculitic and paraneoplastic disorders. Lip minor salivary gland biopsy showed a nonspecific inflammatory pattern not suggestive of the Sjogren's disease. We performed a sural nerve biopsy which was indicative of a lymphocytic infiltration, however we did not observe a typical vasculitic pattern. An elevated high-titer immunoglobulin-G4 (IgG4) was found on follow-up laboratory evaluations. Considering the diagnosis of IgG4-related neuropathy, pulse corticosteroid treatment was initiated which was followed with a dramatic response. Conclusion: IgG4 related neuropathy are a recently recognized pathologic entity and should be considered as a differential diagnosis of probable immune-mediated neuropathic disorders with unknown etiology.

\section{P297}

A Patent Review of Therapeutic Potential of Panax Ginseng and its Constituents, Ginsenosides and Gintonin, in Neurological and Neurodegenerative Disorders (2010-2019)

\section{Fatemeh Rajabian, Maryam Rameshrad, Hossein Hosseinzadeh}

Department of Pharmacodynamics and Toxicology, Faculty of Pharmacy, Mashhad University of Medical Sciences, Mashhad, Iran

The Neuroscience Journal of Shefaye Khatam, 2019; 7(3): P297

Introduction: Neurodegenerative diseases are the consequence of slowly progressive loss of neurons and axons in the central nervous system, which can lead to cognition, behavior, and motor dysfunctions. The toxic effects of neuropathological features of these disorders on synapses, mitochondria, and axonal transport result in disruption of the integrity of brain functions indicated as dysregulation of neurotransmitters such as glutamate. Ginseng, Panax ginseng, has been used for various diseases and proven its great efficacy in managing central nervous system diseases. This review provides a summary of recent advances in the patent reports (20102019) involving therapeutic application of pharmaceutical formulations and compositions containing ginseng and its active constituents for the treatment of neurological disorders. Additionally, the patents on ginseng, ginsenosides, gintonin, and ginsenoside derivatives targeting hallmarks of neurodegenerative diseases have been included in this review. The literature review was provided using multiple search engines including Google Patent, Espacenet and US Patent in the field of neurodegenerative diseases, Alzheimer's disease, Parkinson's disease, cognitive, and neurological disorders. The gath- 
ered data represented outstanding merits of ginseng in treatment of neurodegenerative and neurological disorders. These effects have been mediated by neurogenesis, anti-apoptotic and antioxidant properties, inhibition of mitochondrial dysfunction, receptor-operated $\mathrm{Ca}^{2+}$ channels, amyloid beta aggregation, and microglial activation as well as neurotransmitters modulation. However, these compounds have limited clinical application of them for the prevention or treatment of neurodegenerative and neurological disorders. This might be due to incomplete data on their clinical pharmacokinetic and toxicity properties, and limited economic investments. Conclusion: Combination therapies have been expected to be efficacious in the prevention and treatment of neurodegenerative diseases and minimize side effects and subsequently provide better patient compliance.

\section{P298}

The Role of Therapy Drug Using Rapamycin in Improvement of Multiple Sclerosis Patients: A Systematic Review

Mina Babaei, Mohammad Reza Khojasteh

Mashhad Branch, Islamic Azad University, Mashhad, Iran

The Neuroscience Journal of Shefaye Khatam, 2019; 7(3): P298

Introduction: Multiple Sclerosis (MS) typically is classified as an autoimmune disease, which is affected by about one million people in the United States in 2018. MS disease damages the Myelin Neurons. The rapamycin molecules control the mammalian target of rapamycin (MTORS) of the immune system. In this review, the effects of the rapamycin drug on the treatment of MS patients is investigated by reviewing the published articles available in PubMed and google scholar database since 1999, after accepting rapamycin as a drug by Food and Drug Administration (FDA). By searching keywords Rapamycin, Multiple Sclerosis, Therapy in PubMed Total 149 articles were found that the title of 22 of them was related to our purpose. 6 articles were chosen by reading the abstracts. The same process was done using the Google Scholar site. While 19800 results were found only 5 of them had related title, and among 3 of them having the related abstract article. All of the review articles have mentioned minor side effects of the rapamycin such as a drop in blood sugar level, Skin cancer. It is mentioned in 3 articles that hypothesized MS inflammation and demyelination have decreased by using rapamycin. Another article is showed that the rapamycin suppresses the performance of $\mathrm{T}$ effector. Rapamycin reduces the level of IFN-y in the immune system. In addition, 2 articles have explained the repair of Myelin Neuron by rapamycin. The author of one the articles have examined biochemical and hematological parameters in MS Patients reported Minimum and maximum average volume platelet were lowered. Conclusions: Based on the articles, the rapamycin has positive effects on the remedy of MS patients confirmed and it can be advised by the doctors.
Publisher: Shefa Neuroscience Research Center Layout Designing \& Publication:

Shefa Neuroscience Research Center

Copyright (C) 2019 by Shefa Neuroscience

Research Center

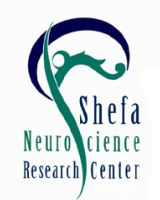

Shefa Neuroscience Research Center 

1. Ghaemi A, Alizadeh L, Babaei S, Jafarian M, Khaleghi Ghadiri M, Meuth SG, et al. Astrocytemediated inflammation in cortical spreading depression. Cephalalgia. 2018; 38(4): 626-38.

2. Ghaffarian N, Mesgari M, Cerina M, Göbel K, Budde T, Speckmann EJ, et al. Thalamocortical-auditory network alterations following cuprizone-induced demyelination. J Neuroinflammation. 2016; 13(1): 160. doi: 10.1186/s12974-016-0629-0.

3. Ghaemi A, Sajadian A, Khodaie B, Lotfinia AA, Lotfinia M, Aghabarari A, et al. Immunomodulatory Effect of Toll-Like Receptor-3 Ligand Poly I:C on Cortical Spreading Depression. Mol Neurobiol. 2016; 53(1): 143-54.

4. Sahab Negah S, Oliazadeh P, Jahanbazi JahanAbad A, Eshaghabadi A, Samini F, Ghasemi S, et al. Transplantation of human meningioma stem cells loaded on a self-assembling peptide nanoscaffold containing

\section{منابع}

IKVAV improves traumatic brain injury in rats. Acta Biomater. 2019; 92: 132-44.

5. Vakilzadeh G, Khodagholi F, Ghadiri T, Ghaemi A, Noorbakhsh F, Sharifzadeh M, et al. The Effect of Melatonin on Behavioral, Molecular, and Histopathological Changes in Cuprizone Model of Demyelination. Mol Neurobiol. 2016; 53(7): 4675-84.

6. Jahanbazi Jahan-Abad A, Sahab Negah S, Hosseini Ravandi H, Ghasemi S, Borhani-Haghighi M, Stummer W, et al. Human Neural Stem/Progenitor Cells Derived From Epileptic Human Brain in a Self-Assembling Peptide Nanoscaffold Improve Traumatic Brain Injury in Rats. Mol Neurobiol. 2018; 55(12): 9122-38.

7. Sajadian A, Esteghamat S, Karimzadeh F, Eshaghabadi A, Sieg F, Speckmann EJ, et al. Anticonvulsant effect of neural regeneration peptide 2945 on pentylenetetrazolinduced seizures in rats. Neuropeptides. 2015; 49: 1523. 